\title{
THE
}

\section{Basaltic Volcaniclastics from the Challenger Deep Forearc Segment, Mariana Convergent Margin: Implications for Tectonics and Magmatism of the Southernmost Izu-Bonin-Mariana Arc}

\author{
Robert J. Stern \\ Minghua Ren \\ Katherine A. Kelley \\ University of Rhode Island, kelley@uri.edu \\ Yasuhiko Ohara

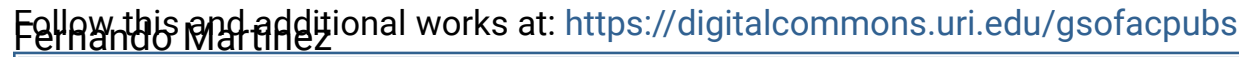

The University of Rhode Island Faculty have made this article openly available.

Please let us know how Open Access to this research benefits you.

This is a pre-publication author manuscript of the final, published article.

Terms of Use

This article is made available under the terms and conditions applicable towards Open Access Policy Articles, as set forth in our Terms of Use.

\section{Citation/Publisher Attribution}

Stern, Robert J.; Ren, Minghua; Kelley, Katherine A.; Ohara, Yasuhiko; Martinez, Fernando; Bloomer, Sherman H. (2014). "Basaltic volcaniclastics from the Challenger Deep forearc segment, Mariana convergent margin: Implications for tectonics and magmatism of the southernmost Izu- Bonin- Mariana arc." Island Arc. 23(4): 368-382.

Available at: http://onlinelibrary.wiley.com/doi/10.1111/iar.12088/abstract

This Article is brought to you for free and open access by the Graduate School of Oceanography at DigitalCommons@URI. It has been accepted for inclusion in Graduate School of Oceanography Faculty Publications by an authorized administrator of DigitalCommons@URI. For more information, please contact digitalcommons-group@uri.edu. 


\section{Authors}

Robert J. Stern, Minghua Ren, Katherine A. Kelley, Yasuhiko Ohara, Fernando Martinez, and Sherman H. Bloomer 


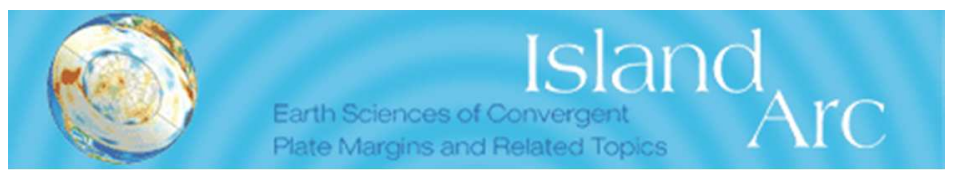

\section{Basaltic volcaniclastics from the Challenger Deep forearc segment, Mariana convergent margin: Implications for tectonics and magmatism of the southernmost IBM arc}

\begin{tabular}{|r|l|}
\hline Journal: & Island Arc \\
\hline Manuscript ID: & Draft \\
\hline Danuscript Type: & Research Article \\
\hline Complete List of Authors: & $\begin{array}{l}\text { Stern, Robert; U Texas at Dallas, Geosciences } \\
\text { Ren, Minghua; U Nevada Las Vegas, Geoscience } \\
\text { Kelley, Katherine; University of Rhode Island, GSO } \\
\text { Ohara, Yasuhiko; Hydrographic and Oceanographic Department of Japan, } \\
\text { Martinez, Fernando; University of Hawaii, Hawaii Institute of Geophysics } \\
\text { and Planetology } \\
\text { Bloomer, Sherman; Oregon State U, Geosciences }\end{array}$ \\
\hline Key words: & Subduction, Mariana Arc, basalt, Challenger Deep \\
\hline
\end{tabular}


Basaltic volcaniclastics from the Challenger Deep forearc segment, Mariana convergent margin: Implications for tectonics and magmatism of the southernmost IBM arc

Robert J. Stern ${ }^{1}$, Minghua Ren ${ }^{2}$, Katherine A. Kelley $^{3}$, Yasuhiko Ohara ${ }^{4}$, Fernando Martinez ${ }^{5}$, Sherman H. Bloomer ${ }^{6}$

${ }^{1}$ Geosciences Dept., U. Texas at Dallas, 800 W. Campbell Road, Richardson TX 75080 USA

2 Dept. Geosciences, University of Nevada, Las Vegas, Las Vegas, NV 89154-4010, USA

${ }^{3}$ Graduate School of Oceanography, University of Rhode Island, Narragansett Bay Campus, Narragansett, Rhode Island, USA

${ }^{4}$ Japan Agency for Marine-Earth Science and Technology, Natsushima, Yokosuka and Hydrographic and Oceanographic Department of Japan, Koto-ku, Tokyo, Japan

${ }^{5}$ Hawai'i Institute of Geophysics and Planetology, SOEST, University of Hawai'i at Manoa, Honolulu, Hawaii, USA

${ }^{5}$ Geosciences Department, Oregon State University, 128 Kidder Hall, Corvallis, Oregon 97331, USA

* Corresponding author ristern@utdallas.edu, Tel. +001-972-883-2442 Fax +001-972883-2537 


\section{ABSTRACT}

Convergent margin igneous activity is generally limited to $100-200 \mathrm{~km}$ from the trench except where spreading ridges are subducted or in association with SubductionTransform Edge Propagators (STEP faults). The southernmost Mariana forearc, facing the Challenger Deep, subducts Mesozoic seafloor and is not in a STEP fault setting but includes at least one site where tholeiitic basalts recently erupted close to the trench, the SE Mariana Forearc Rift (SEMFR). Here we present evidence of young basaltic volcanism from another site $\sim 100 \mathrm{~km}$ west of SEMFR. Shinkai 6500 diving during YK1308 (Dive 1363) recovered volcaniclastics from $\sim 5.5$ to $6 \mathrm{~km}$ deep in the inner wall of the Mariana Trench, $50 \mathrm{~km}$ NE of the Challenger Deep. Glassy fragments are tholeiitic basalts similar to MORB except for much higher contents of magmatic water $\left(\sim 2 \% \mathrm{H}_{2} \mathrm{O}\right.$ vs. $<0.2 \% \mathrm{H}_{2} \mathrm{O}$ in $\left.\mathrm{MORB}\right)$ and spikes in trace element diagrams at $\mathrm{Rb}-\mathrm{Cs}-\mathrm{Ba}$, $\mathrm{K}, \mathrm{Pb}$, and $\mathrm{Sr}$. Dive 1363 basalt glasses are similar to basalts from SEMFR erupted near the trench and to basalts of the Mariana Trough backarc basin. Basalt fragments and palagonitized matrix dominate the three samples we studied, but small xenocrysts and xenoliths derived from mantle peridotite and Neogene volcanics are also present, probably torn from the vent walls. Dive 1363 hyaloclastites erupted at 3-6 km water depth accompanied by vigorous degassing of volatiles, most likely $\mathrm{CO}_{2}$. These results provide further evidence that the forearc adjacent to the Challenger Deep has been invaded by asthenospheric mantle and is unusually weak. The weakness of the overriding plate may result in weak coupling between the subducting Pacific plate and overriding Mariana plate and this may be partly responsible for the great depth of the Challenger Deep. 
Keywords: Subduction, Mariana Arc, basalt, Challenger Deep 


\section{INTRODUCTION}

The southernmost part of the $\sim 1500 \mathrm{~km}$-long Mariana arc system - where the Mariana Trench bends sharply west (Fig. 1a) - is a region that is tectonically active and poorly understood. Here, the Pacific Plate subducts almost orthogonally beneath the easternmost Philippine Sea (Mariana Plate) at about $30 \mathrm{~mm} /$ year (Bird 2003). Because the plate boundary trends E-W here, it cuts across the southern part of the Mariana Trough, an actively spreading back-arc basin (BAB; Fig. 1a). This combination of strong convergence and extension is associated with the deepest point on Earth's solid surface, the Challenger Deep. It also causes the adjacent part of the Mariana Trough just north of the Trench to be seismically and magmatically active and to deform rapidly and complexly. We know from GPS studies that the southernmost Marianas (Fig. 1b) is the most rapidly deforming part of the $3500 \mathrm{~km}$ long Izu-Bonin-Mariana (IBM) arc system (Kato et al. 2003), but we are only beginning to understand how deformation and magmatism are distributed over this deeply submerged region.

Tectonics of the southernmost Marianas have a strong influence on the Mariana Trough BAB. Fryer (1995) first noted that the Mariana Trough had a different expression south of $\sim 14^{\circ} \mathrm{N}$ and concluded that this reflected a different tectonic style in this region relative to that farther north. For most of its $\sim 1200 \mathrm{~km}$ length, the Mariana Trough opens slowly E-W along a ridge system with slow-spreading axial valley morphology producing a variable but somewhat thin $(3.5-4 \mathrm{~km})$ volcanic crust estimated from gravity and bathymetry values (Kitada et al, 2006). South of $\sim 14^{\circ} \mathrm{N}$ the spreading center bends increasingly westward and develops a fast-spreading axial-high morphology, although actual spreading rates are not likely high (Martinez et al., 2000). Gravity data suggest 
somewhat thicker crust in this area $(6.7 \mathrm{~km})$ (Kitada et al., 2006). The complexly deformed region - which we call the Southernmost Mariana Trough-Trench Complex (SMTTC) - is delimited on the east by the West Santa Rosa Bank Fault (WSRBF) Fryer et al., (2003). WSRBF can be traced as a $\square 5 \mathrm{~km}$ scarp south of $13^{\circ} \mathrm{N}$, diminishing in relief northwards until it is replaced by a northeast-trending fault scarp south of Tracey Seamount (Fig. 1b). The northern limit of the region affected by SMTTC tectonics lies $\sim 13^{\circ} 30^{`} \mathrm{~N}$, about where the Mariana volcanic front loses definition southward. This is also about where the BAB spreading center changes from an axial rift in the north into the inflated Malaguana-Gadao Ridge (MGR; Fig. 1b), which is underlain by the only known magma chamber in the Mariana Trough BAB (Becker et al. 2010). The Southern Mariana Forearc Ridge (Fig. 1B) separates the Mariana Trough to the north from the trench and Challenger Deep to the south; where this ridge has been sampled, it is composed of Miocene arc volcanics (Ohara et al., in preparation).

More evidence that the SMTTC is unusually active comes from how the locus of arc volcanism is disrupted in this region. For $\sim 3000 \mathrm{~km}$ north from Tracey seamount $\left(\sim 13^{\circ} 40^{`} \mathrm{~N}\right)$ all the way to Japan, discrete and well-developed volcanoes of the IBM active arc define a pronounced string of stratovolcanoes that is separated from the trench by a broad ( $150-200 \mathrm{~km}$ wide) forearc. As is characteristic for other magmatic arcs, IBM arc volcanoes are typically found $\sim 100-150 \mathrm{~km}$ above the subducted Pacific Plate. Such a line of discrete, long-lived volcanoes is typical for mature convergent margins and is known as the 'magmatic front' (Matsuda \& Uyeda, 1971). The magmatic front marks where fluids and sediment melts released from the subducting plate trigger melting of convecting asthenosphere, and arc volcanoes build up over time where these melts rise to 
the surface. South of Tracey seamount, the line of arc volcanoes is poorly defined (Fig. 1B, Stern et al., 2013), despite the fact that the southern Mariana Trough is underlain by a subducted slab that can be traced to $150 \mathrm{~km}$ depth (Gvirtzman \& Stern, 2004). Poor definition of the magmatic arc SW of $13^{\circ} 40^{\prime} \mathrm{N}$ reflects tectonic instability in the SMTTC. Multiple sites of extension frequently divert the supply of arc magmas, not allowing magma supply to focus beneath discrete volcanoes so that these can grow to become large stratovolcanoes, as is seen for the arc to the north (Stern et al., 2013). Complex deformation in the SMTTC reflects three interacting causes: 1) subduction of the Pacific plate, which induces asthenospheric convection at the same time that it supplies magma and fluids to the overlying mantle, causing flux melting; 2) BAB opening, which keeps lithosphere thin and causes decompression melting; and 3) rapid rollback of a narrow, short slab, which adds trench-normal extensional stresses to the overriding plate. Gvirtzman and Stern (2004) concluded that the plate-coupling zone along the Challenger Deep forearc segment was unusually narrow, only $50 \mathrm{~km}$ wide compared to $\sim 150 \mathrm{~km}$ wide beneath the forearc farther north. The unusually narrow plate coupling zone allows convecting asthenosphere to penetrate closer to the trench than is found for other forearcs, and this allows asthenosphere to be fluxed by shallow, slabderived hydrous fluids and melt (Ribeiro et al., 2013a, b). The result is an unusually weak forearc that is volcanically active much closer to the trench than normally occurs. It is not easy to identify where igneous activity occurs in this complexly deforming region. The Southeast Mariana forearc rift (SEMFR) marks one such region of forearc igneous activity, floored by 2.7-3.7 Ma low-K tholeiitic basalts (Ribeiro et al., 2013a, b). SEMFR lavas were produced by partial melting of a BAB-like mantle source, 
metasomatized by sediment melt and aqueous fluids released from dehydration of the subducted oceanic crust. SEMFR melts were probably generated when the Mariana Trough backarc basin (BAB) first began to open in this region. But where else does igneous activity occur in this enigmatic and complexly-deforming region, and how close to the trench does igneous activity occur? In this contribution we present new evidence that MORB-like basalts recently erupted very close to the trench, $\sim 100 \mathrm{~km}$ west of SEMFR.

\section{SAMPLE COLLECTION}

Regional multibeam bathymetry in the area was obtained by US Law of the Sea mapping project (Armstrong, 2011) as well as on R/V Yokosuka (Fig.2 A). In Dec. 2011 to Jan. 2012 R/V Thomas G Thompson obtained two swaths of deep-towed ( 500 m altitude) IMI-30 sidescan sonar imagery over the area (Martinez et al., 2012, Fig. 2B). Samples were collected during dive \#1363 of the manned submersible Shinkai 6500 on Sept. 10, 2013 as part of JAMSTEC research cruise of R/V Yokosuka (YK1308). The dive site was located $\sim 11^{\circ} 38^{\prime} \mathrm{N}, 143^{\circ} \mathrm{E}, \sim 30 \mathrm{~km}$ north of the trench axis, $\sim 7.5 \mathrm{~km}$ west of the Shinkai Seep Field (Ohara et al., 2012), and 60 km ENE of the Challenger Deep (Fig. 2). The dive traversed north up the inner wall of the Mariana Trench from $6094 \mathrm{~m}$ to $5584 \mathrm{mbsl}$ and was intended to search for additional forearc seeps and communities. Previous studies suggest that the Moho is exposed at 5500 mbsl near the study area, so we expected to recover peridotites. 18 samples were collected during this dive, consisting of peridotites and moderately lithified volcaniclastic sediments (hyaloclastities), composed of sand-sized, reddish-brown matrix with pieces of basaltic 
glass up to $2 \mathrm{~cm}$ across (Fig. 3). Three samples of volcaniclastic sediment (R05, R06, and R15; see Fig. 2 for locations) from Shinkai dive 1363 were studied. Dive 1364 continued the traverse up the slope (5608 - 5197 m; Fig. 2A) and encountered similar volcanic-rich sediments but these were much less common than peridotites.

The samples that we studied are all basaltic volcaniclastics. Our chemical studies focus on glass fragments but we also examined the texture and composition of the volcaniclastic matrix (Fig. 4). The matrix shows no lamination or bedding, is poorly sorted and well indurated, and we interpret the samples as fragments from a volcaniclastic, bottom-hugging gravity flow. These probably moved from the eruption site as laminar mass flows (Fischer 1984) and must have been deposited downhill from their eruption vent. We have no constraints about how many different pyroclastic flows were sampled. Because the three samples we examined are similar in appearance and contain basaltic glass of very similar composition, they could be from the same flow. We have no constraints on the width or thickness of the volcaniclastic flow(s).

The volcaniclastic matrix is full of rock and mineral fragments of various shapes and sizes, including delicate fragments such as those highlighted with yellow "D" in Fig. 4. Such delicate fragments are unlikely to have suffered much buffeting from grain-to-grain contacts during transport, and must have been supported during transport downslope by the strength and buoyancy of the matrix. We infer that the volcaniclastic deposit formed as a submarine mudflow or lahar as it moved downslope from the eruption site. One sample (1364-R03) is a harzburgite with a $4 \mathrm{~cm}$ thick semi-lithified muddy volcanic sandstone rim, suggesting that the harzburgite may have been a clast dislodged in the vent or picked up during volaniclastic flow. Although palagonite is common in the 
matrix, the glass fragments are fresh and unaltered. Manganese coating on volcaniclastic samples is thin to nonexistent, and we infer from this and freshness of glass fragments that the flow occurred sometime in the last million years.

\section{ANALYTICAL METHODS}

Glass fragments, microlites in glass fragments, and matrix components were studied. Rock samples were examined using electron backscatter imaging and this allowed us to determine major element compositions of mineral phases and glass fragments using the electron microprobe at U Nevada Las Vegas using a JAX8900 electron microprobe analyzer equipped with four wavelength-dispersive spectrometers. Basaltic volcaniclastics and matrix were placed in 1" epoxy mount and polished and carboncoated prior to analyses. Elements $\mathrm{Si}, \mathrm{Ti}, \mathrm{Al}, \mathrm{Cr}, \mathrm{Fe}, \mathrm{Mn}, \mathrm{Mg}, \mathrm{Ca}, \mathrm{Na}, \mathrm{K}, \mathrm{P}, \mathrm{F}, \mathrm{Cl}$ and $\mathrm{S}$ were analyzed at $15 \mathrm{keV}$ acceleration voltage, beam current $10 \mathrm{nA}$, defocused beam of 20 micrometers for glasses, with a peak counting time of 10 seconds for $\mathrm{Na}$ and 30 seconds for other elements. For mineral grains, beam condition were $15 \mathrm{keV}$ acceleration voltage, $20 \mathrm{nA}$ beam current, defocused beam of 10 micrometers, with a peak counting times of 20 seconds for $\mathrm{Na}$ and 30 seconds for other elements. Since the high-Si glass fragments are about 10 to 15 micrometers across, the beam size had to be set at 10 micrometers, possibly leading to loss of Na. Calibration standards used for glass analyses were: Smithsonian VG2 basaltic glass for $\mathrm{Si}$ and $\mathrm{Ca}$, almandine for $\mathrm{Al}$, chromite for $\mathrm{Cr}$, ilmenite for $\mathrm{Ti}$, pyrope for $\mathrm{Fe}$ and $\mathrm{Mg}$, rhodonite for $\mathrm{Mn}$, albite for $\mathrm{Na}$, microcline for $\mathrm{K}$, apatite for $\mathrm{P}$, fluorite for $\mathrm{F}, \mathrm{AgCl}$ for $\mathrm{Cl}$, and barite for $\mathrm{S}$. For high Si glass, Smithsonian 
tektite glass was used for Si calibration. For mineral grains in matrix, $\mathrm{Si}, \mathrm{Al}$, and $\mathrm{Ca}$ used Smithsonian plagioclase for calibration.

Water and $\mathrm{CO}_{2}$ contents in one chip from each of 3 samples were determined by Fourier Transform Infrared (FTIR) spectroscopy at the Graduate School of Oceanography, University of Rhode Island, using a Thermo Nicolet iS50 bench FTIR coupled with a Continuum IR microscope. The sample area was purged with dry, $\mathrm{CO}_{2^{-}}$ free air to minimize atmospheric interferences, and analytical conditions used a custom aperture that varied from $100 \times 100 \mu \mathrm{m}$ to $60 \times 60 \mu \mathrm{m}$, depending on crystallinity of the matrix glass. Data were collected in transmission using a $250 \mu \mathrm{m}$ MCT-A detector, and reduced following methodologies outlined by Kelley and Cottrell, 2012. Owing to the microcrystalline nature of these glasses, thin wafer preparation $(30-40 \mu \mathrm{m})$ was required for two glasses in order to expose enough optically clear glass for volatile analysis, rendering dissolved $\mathrm{CO}_{3}{ }^{2-}$ below detection.

Trace element abundances were determined by Laser Ablation InductivelyCoupled Plasma Mass Spectrometry (LA-ICP-MS) at the Graduate School of Oceanography, University of Rhode Island, using a Thermo X-Series 2 quadrupole ICPMS coupled with a New Wave UP213 Nd-YAG laser ablation system. Data were collected using $80 \mu \mathrm{m}$ spots and a $5 \mathrm{~Hz}$ repeat rate, normalized to ${ }^{43} \mathrm{Ca}$ as the internal standard, and calibrated against 8 natural-composition reference glasses from the USGS and MPI-DING series (BCR-2G, BIR-1G, BHVO-2G, KL2-g, ML3B-g, StHls-g, T1-g, GOR-132-g), following methods outlined by Kelley et al. (2003) and Lytle et al. (2012).

\section{RESULTS}


Complete microprobe analyses are provided in Supplementary Documents 1 (major element compositions of glass fragments), 2 (compositions of minerals in glass), and 3 (compositions of small minerals and rock fragments in matrix). These results are discussed in order of glass, minerals in glass, and minerals in matrix below. 153 major element analyses of 14 fragments were carried out for R05, 131 analyses of 13 fragments for R06, and 145 analyses of 13 fragments from R15 were carried out, for a total of 429 analyses. These analyses gave very similar compositions, indicating that the glass fragments are basalt (Table 1). Analytical totals are consistently $\sim 97.5-98 \%$, suggesting the presence of considerable ( $\sim 2$ wt. \%) water and other magmatic volatiles, similar to what is reported for BAB tholeiites (Kelley et al., 2006). The composition of these glasses are otherwise remarkably MORB-like (Table 2), especially in terms of low abundances of incompatible major elements: $\mathrm{TiO}_{2}, \mathrm{Na}_{2} \mathrm{O}$, and $\mathrm{K}_{2} \mathrm{O}$. These characteristics of Dive 1363 basaltic glasses are comparable to basaltic rocks from the SE segment of the SE Mariana Forearc rift (SE-SEMFR) to the east, which erupted in a comparable near-trench position to that of the Dive 1363 basaltic glass samples (Fig. 1B, Table 1; Ribeiro et al., 2013a, b).

Abundances of magmatic $\mathrm{H}_{2} \mathrm{O}$ and $\mathrm{CO}_{2}$ in glass fragments are also presented in Table 1.

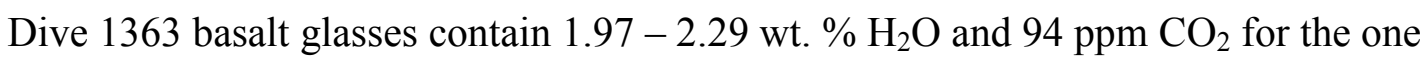
sample with detectable dissolved $\mathrm{CO}_{2}{ }^{2-}$. Volatile $\left(\mathrm{H}_{2} \mathrm{O}-\mathrm{CO}_{2}\right)$ saturation pressure for this sample, as modeled using VolatileCalc (Newman \& Lowenstern, 2002) is 642 bars, consistent with a hydrostatic eruption depth of $6545 \mathrm{~m}$, which is near the collection depth of the sample. The fact that the carbon dioxide is not completely outgassed suggests that magmatic water did not outgas significantly and that the mean of 2.1 wt. $\% \mathrm{H}_{2} \mathrm{O}$ is a 
useful approximation of the water content in the basaltic magma when it erupted. This is much higher $\mathrm{H}_{2} \mathrm{O}$ contents than found in most MORB glasses and is the single most important way that MORB and Dive 1363 glasses differ; primitive NMORB contains $0.15 \% \mathrm{H}_{2} \mathrm{O}$ (Michael 1995), less than $10 \%$ of what the 1363 basalts contain. Dive 1363 glasses are very similar to the one sample of SE-SEMFR basalt glass analyzed for water and $\mathrm{CO}_{2}$ (Ribeiro et al. submitted)

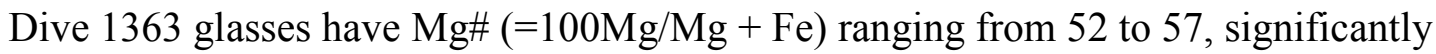
lower than $\mathrm{Mg} \# \sim 65$, expected for unfractionated, primitive basalts. Dive 1363 glass fragment Mg\# is similar to that of MORB and SE-SEMFR basalt, all of which show similar extents of fractionation. CIPW norms (Table 1) indicate that Dive 1363 basalts are quartz-normative tholeiites, similar to SE-SEMFR basalts but differing from typical MORB, which is often olivine-normative basalt.

Trace element concentrations for the three Dive 1363 basaltic glass samples are listed in Table 2, along with some key trace element ratios and mean compositions of SESEMFR basalt and MORB. Dive 1363 basalts have chondrite-normalized Rare Earth Element (REE) patterns with concave-downward patterns and modest light REE depletions (Fig. 5). These REE patterns are very similar to those of MORB and SESEMFR basalts. All three Dive 1363 glasses show maxima in the middle REE (Nd - Gd) - also like SEMFR and MORB; and all three show a modest decrease in the heavy REE, from $\mathrm{Tb}$ to $\mathrm{Lu}$. Extended trace element patterns (spider diagrams; Fig. 6) emphasize strong similarities and subtle differences between Dive 1363 glasses and SE-SEMFR basalts on the one hand and MORB on the other. One significant difference is that Dive 1363 basalts have a modest negative $\mathrm{Nb}$-Ta anomaly $(\mathrm{Th} / \mathrm{Nb}=\sim 0.1)$ whereas MORB 
generally does not $(\mathrm{Th} / \mathrm{Nb}=0.08)$. In addition, Dive 1363 samples have a somewhat higher ratio of fluid-mobile incompatible elements (e.g., $\mathrm{Rb}$ ) relative to similarly incompatible but fluid-immobile elements (e.g., Zr) than do MORB (Table 2). There are also indications from lower Ti/V that Dive 1363 magmas originated from a somewhat more oxidized mantle source region than do most MORB (Table 2).

Small crystals of plagioclase and clinopyroxene occur in the glasses. Plagioclase in all three samples is mostly bytownite. 27 analyses of plagioclase in R05 yields a range of An67-83, mean $=$ An74.7 \pm 3.6 ( 1 standard deviation $) .26$ analyses of plagioclase in R06 yields a range of An68-95, mean $=$ An77.8 \pm 9.46 analyses of plagioclase in R15 yields a range of An67-88, mean=77.2 \pm 7 . Clinoyroxene in all three samples is anhedral augite with similar compositions. 18 analyses of one clinopyroxene in R05 yielded Wo43.4 \pm 2.3 En46.5 \pm 1.7 Fs9.2 \pm 1.2 Ac0.9 \pm 0.16 . Eight analyses of one clinopyroxene in R06 yielded a mean of Wo43 \pm 2 En47 \pm 2 Fs9 \pm 1 Ac0.8 $\pm 0.2 . \square$ Twelve analyses of clinopyroxene in R15 yielded a mean of Wo42 \pm 2 En $47 \pm 2$ Fs10 \pm 0.75 Acc $0.9 \pm 0.35$. $\square$ The compositions of plagioclase and clinopyroxenes in all three samples are essentially identical. $\square \square$ These mineral compositions indicate crystallization from fractionated magma. There is one anhedral amphibole and one anhedral ilmenite found in one R15 basaltic glass fragment, the amphibole can be classified as Tschermakite.

Rock and mineral fragments in the volcaniclastic matrix include olivine, orthopyroxene, serpentine, epidote, amphibole, magnetite, clinopyroxene, plagioclase, quartz, basaltic glass, and high-Si glass. Electron microprobe analyses are listed in Supplementary Document 3. 
71 fragments in R05 matrix were analyzed. We idenitified four olivines (Fo 90.6-91.6), one orthopyroxene (Wo5.3 En71.4 Fs23.2 Ac0.2), one epidote, one amphibole (Magnesio-hornblende), four magnetite, one high-Si glass, 26 clinopyroxenes, 23 plagioclases, and eight basaltic glasses. Excepting 4 sodic plagioclases (An6-49), all other plagioclases (An 67-91), clinopyroxenes (En38-45), and basaltic glasses are similar to those in the large basaltic glass and the included plagioclase and clinopyroxene. Twenty six fragments were analyzed in the R06 matrix. There are two olivines (Fo78 and Fo81), one serpentine, one quartz, seven clinopyroxene (En 38-47), six plagioclase (An73-86), nine basaltic glasses. The olivine is different from olivines from R05 and R15 samples. All plagioclases, clinopyroxenes, and basaltic glasses are similar to the large basaltic glass and the included plagioclase and clinopyroxene.

Forty five fragments were analyzed in R15 matrix. There are seven olivine (Fo90-92), six serpentine, one epidote, two amphibole, eight clinopyroxene (En41-45), eight plagioclase, 10 basaltic glass, and one high-Si glass. R15 olivine $($ Fo $>90)$ is identical to R05 olivine, but different from R06 olivine (Fo78-81). R15 matrix clinopyroxene (En4145 ) is slightly different from clinopyroxene in basaltic glass, the matrix clinopyroxene have higher $\mathrm{Al}_{2} \mathrm{O}_{3}$ and $\mathrm{TiO}_{2}$ than clinopyroxene in basaltic glass. There are two high $\mathrm{Na}$ plagioclase grains (An43-56), other plagioclases (An73-85) are similar to the plagioclase in basaltic glass. There are two basaltic glass grains have higher $\mathrm{MgO}$ (15.4-16.6wt\%) and lower $\mathrm{Al}_{2} \mathrm{O}_{3}(4.9-6.2 \mathrm{wt} \%)$ than other basaltic glasses, which are similar to the large basaltic glasses $\left(\mathrm{Al}_{2} \mathrm{O}_{3} \sim 16-17 \mathrm{wt} \%, \mathrm{MgO} \sim 4-6 \mathrm{wt} \%\right)$.

\section{DISCUSSION}


The MORB-like composition of the YK-1308 6K1363 glasses that we have analyzed indicate derivation from melting of oceanic asthenosphere, but it is surprising that such melts were generated so close to the deepest trench on Earth.

These results are intriguing but more work is needed to determine the extent of young BAB igneous activity near the Challenger Deep before we can understand its significance. Here we briefly discuss three implications of our results: 1) What was the eruptive style and where did the eruption occur? 2) What are the petrogenetic implications of Dive 1363 basalts? and 3) What are the implications of our results for future studies of the region?

1) Eruption style and vent location:

The three rock samples that we studied formed by a frothy eruption of basaltic magma that was broadly MORB-like but with high water and which may have contained high $\mathrm{SO}_{2}$ and/or $\mathrm{CO}_{2}$ contents prior to eruption. This magma degassed vigorously as it erupted. We have no direct information about where the eruption occurred, but because these are laminar density flows (lahars), they must have originated somewhere upslope along the inner trench wall to the north of where they were collected. They could not have originated from the north side of the S. Mariana Forearc Ridge (Fig. 1B). The eruption site may have occurred on the bathymetric highs north of the dive site ('*' in Fig. 1b and 7). Regional HMR-1 sonar backscatter imagery (Fryer et al., 2003) over the region (Fig. 7) shows no obvious volcanic features around the dive site, but the entire trenchward slope is characterized by high backscatter, indicating steep slopes of lightly sedimented basement. There is a low backscatter region surrounding the local forearc 
high labeled with an “*” in Fig. 7. We don't know what seafloor materials are exposed in the low backscatter region but it could be volcaniclastics. Deep towed IMI-30 sidescan sonar imagery of the dive area (Fig. 2) also does not give clear indications of young local volcanic structures, as imaged and sampled farther to the east in the SEMFR area (Martinez et al., 2012). Basement morphology indicates generally low sediment thickness with local ponds (light shaded areas, Fig. 2B). Imaged lobe-like morphologies may be landslide or debris flow fronts.

We note that the volatile saturation pressure of the glass from R15, which has reliable $\mathrm{CO}_{2}$ contents, suggests a much higher eruption pressure, closer to $6 \mathrm{~km}$. This suggests that the magma erupted so rapidly that it did not have time to fully degas before quenching, or the vent is closer to the dive site than we propose above. The preservation of delicate fragments also suggests a nearby eruption site. Clearly more work is needed to identify the eruption site.

The eruption was sufficiently violent to be something like a version of a deepwater $(>3 \mathrm{~km})$ 'Strombolian-style" eruption. At pressures lower than that for mixed $\mathrm{SO}_{2}-\mathrm{CO}_{2}-\mathrm{H}_{2} \mathrm{O}$ fluid saturation, magmas that are rich in these volatiles are likely to erupt more explosively than will fluid-poor magmas such as normal MORB. Violent deepwater eruptions are poorly known, but such an eruption style is required for fragmenting and quenching the erupting basaltic magma to form glass. Because the ridge to the north is as shallow as $3 \mathrm{~km}$ and samples were collected from as deep as $6 \mathrm{~km}$, the eruption must have occurred at 300-600 bars hydrostatic pressure. Even at such pressures, mixed $\mathrm{SO}_{2}-\mathrm{CO}_{2}-\mathrm{H}_{2} \mathrm{O}$ fluid can be oversaturated in basaltic magmas and $\mathrm{SO}_{2}$ and $\mathrm{CO}_{2}$ can begin to exsolve below the seafloor. The eruption that formed these 
volcaniclastic flows could have been like that recently observed at West Mata volcano in the Lau Basin (Resing et al., 2011), although hydrostatic pressures resisting vesiculation and fragmentation would be significantly greater at the $>3 \mathrm{~km}$ water depth of Mariana forearc eruption than at the $\sim 1100$ m eruption depth of W Mata (300 vs. 110 bars hydrostatic pressure).

Clearly the matrix was derived from several sources, principally basaltic magma but also incorporating fragments of the peridotite and older volcanic substrate. One source was the eruption itself, which provided fragments of basaltic glass and associated plagioclase and clinopyroxene (Fig. 4A, B, C). Contributions from ultramafic sources are also revealed by fragments of Fo90 olivine and serpentinite (Fig. 4B, C). High silica glass and amphibole grains (Fig. 4A, C) probably sample underlying Miocene volcanics. We also identified fragments of high $\mathrm{Si}$ glasses, $\mathrm{SiO}_{2} 71-73 \%$, along with an albite, several grains of andesine, and a few grains of epidote.

It seems likely that the fragments of peridotite xenocrysts and serpentinite fragments found in the volcaniclastic matrix could have been torn off the vent walls accompanying sub-seafloor exsolution of magmatic volatiles. It is also possible that non-basaltic fragments were picked up from rock exposures during lahar flow. Fragmentation was rapid enough and transport distance was short enough that sufficient heat was retained by fragments to partially anneal the deposit after it came to rest.

\section{2) Petrogenetic implications}

As noted above, Dive1363 glasses are broadly MORB-like but with unusually high abundances of magmatic water. Some trace element ratios are MORB-like, for example 
$\mathrm{La} / \mathrm{Nd}=0.42-0.44$. The similarity is also clear from chondrite-normalized Rare Earth Element (REE) patterns (Fig. 5). Major and trace element compositions of the three Dive 1363 samples are remarkably similar to each other and to basalts from SEMFR to the east and to global MORB (All-MORB of Gale et al. 2013).

Dive 1363 basalts show extended trace element patterns (spider diagrams; Fig. 6) that are similar to those of SE-SEMFR basalts to the east. This serves to further emphasize the strong similarities and subtle differences between Dive 1363 glasses and SEMFR basalts on the one hand and MORB on the other. Major element compositions and REE patterns are remarkably similar for Dive 1363, SEMFR, and MOR basalts. One subtle difference with MORB is that Dive 1363 basalts show modest negative $\mathrm{Nb}-\mathrm{Ta}$ anomalies $(\mathrm{Th} / \mathrm{Nb}$ $=\sim 0.1)$ whereas MORB generally does not $(\mathrm{Th} / \mathrm{Nb}=0.08)$. In addition, Dive 1363 basalts have a somewhat higher ratio of fluid-mobile incompatible elements like Rb relative to similarly incompatible but fluid-immobile $\mathrm{Zr}$ than do MORB (Table 2). There are also indications - based on lower Ti/V - that Dive 1363 magmas originated from a somewhat more oxidized mantle source region than do most MORB (Table 2). Ti/V is thought to proxy for mantle oxidation state, with oxidized arc magmas having low Ti/V $(<20)$ compared to higher values for more reduced MORB and OIB (20-100; Shervais, 1982). Mean SEMFR basalt has $\mathrm{Ti} / \mathrm{V}=19$ but the three Dive 1363 basalt glasses have higher and more MORB-like Ti/V of 24-25.

$\mathrm{La} / \mathrm{Nb}=1.4$ was argued by Condie (2001) to distinguish convergent margin lavas (with higher ratios) from basalts formed in other tectonic environments such as MORB and oceanic hotspots. The three samples of Dive 1363 basalt glass have $\mathrm{La} / \mathrm{Nb}=1.31-1.43$, intermediate between All-MORB ( $\mathrm{La} / \mathrm{Nb} \sim 1)$ and mean SEMFR basalt $(\mathrm{La} / \mathrm{Nb}=1.78)$. 
$\mathrm{Nb} / \mathrm{Yb}$ is thought to be a proxy for depletion of the mantle source, $<\sim 1$ for depleted mantle, $>\sim 1$ for undepleted or enriched mantle (Pearce 2008). DIVE 1363 basalts have $\mathrm{Nb} / \mathrm{Yb}=1.05-1.16$, somewhat lower than MORB $(\mathrm{Nb} / \mathrm{Yb}=1.44)$ but somewhat higher than mean SEMFR lavas $(\mathrm{Nb} / \mathrm{Yb}=0.83)$.

$\mathrm{Pb} / \mathrm{Ce}$ is thought to track contributions from subducted sediments to the mantle source, with values approaching 0.5 for arc lavas (Miller et al., 1994), much higher than the $\mathrm{Pb} / \mathrm{Ce}$ of MORB ( 0.04). The three samples of Dive 1363 glass have $\mathrm{Pb} / \mathrm{Ce} \sim 0.08$, much closer to MORB than to arc magmas and indicating a barely detectable contribution from subducted sediments. Plank (2005) argued that Th/La tracked sediment contributions, with mantle $\mathrm{Th} / \mathrm{La}<0.20$ and arc and sediments $\mathrm{Th} / \mathrm{La}>0.20$. The three samples of 1363 basalt, mean SEMFR, and global MORB have indistinguishable $\mathrm{Th} / \mathrm{La}=0.08$, again indicating that subducted sediments contributed negligibly to the source of the 1363 basalt glasses.

3) Implications for future research:

YK1308 6K1363 volcaniclastics represents the second place in the southernmost Mariana forearc where we have found evidence of young basaltic volcanism close to the trench, the other being SEMFR, $130 \mathrm{~km}$ to the east. Igneous activity so close to a convergent plate margin is common only where $\mathrm{BAB}$ spreading ridges intersect the trench at Subduction-Transform Edge Propagators, or STEP faults (Govers \& Wortel, 2005). However, in these setting the lower plate motion at the trench is primarily transcurrent rather than convergent and these sites mark the lateral termination of subduction zones (Govers \& Wortel, 2005). The Challenger Deep forearc segment is not in a STEP fault 
geometry. What it has in common with STEP fault margins is active extension of the upper plate that advects asthenospheric mantle leading to melting and volcanism at the trench. Another difference from STEP fault margins is that upper plate extension at these margins is focused to narrow spreading centers whereas in the southern Mariana margin it is diffuse.

Identification of sites in the inner trench wall that erupt tholeiitic basalt demonstrate that $\mathrm{BAB}$ asthenosphere penetrates unusually far into the forearc and that lithosphere beneath the SMTTC is thin. Gvirtzman and Stern (2005) argued that weak coupling between the downgoing Pacific plate and the overriding Mariana plate allowed the subducting plate to bend and sink more steeply than normally observed, and was an important contributing cause to the great depth of the trench here. Evaluating this possibility should be an important geoscientific research focus for the $21^{\text {st }}$ century and will require interdisciplinary field, laboratory, and theoretical studies of what is happening on both sides of the Challenger Deep. On the Mariana margin, we need to look for more sites of recent volcanism in the inner trench wall. There are likely to be other sites of basaltic volcanism here that are yet to be discovered, and other regions of this forearc should be investigated for signs of volcanic activity. This includes summit regions of individual highs on the Southern Mariana Forearc Ridge as well as the N-S depression at $143^{\circ} 15^{\prime} \mathrm{E}$ (Fig. 1B). In addition, an OBS field program in the SMTTC is needed to define regions of shallow seismic activity, depth to the base of the lithosphere, and the geometry of the downgoing slab. Modeling studies to understand why the Challenger Deep forearc segment is so weak are needed to support these efforts. The Pacific plate south of the trench also needs to be swathmapped and geophysically investigated to see if evidence of 
unusually strong bending exists, such as outer trench normal faults and seismicity.

\section{CONCLUSION}

We have found a second site of young basaltic volcanism in the Challenger Deep forearc segment. Shinkai 6500 diving during YK1308 (Dive 1363) recovered volcaniclastics from $\sim 5.5$ to $6 \mathrm{~km}$ deep in the inner wall of the Mariana Trench, $\sim 50 \mathrm{~km} \mathrm{NE}$ of the Challenger Deep. Abundant fragments of glassy fragments of tholeiitic basalts analyzed from three different samples are compositionally similar to MORB except for much higher contents of magmatic water $\left(\sim 2 \% \mathrm{H}_{2} \mathrm{O}\right.$ vs. $<0.2 \% \mathrm{H}_{2} \mathrm{O}$ in MORB) and spikes in trace element diagrams at $\mathrm{Rb}-\mathrm{Cs}-\mathrm{Ba}, \mathrm{K}, \mathrm{Pb}$, and $\mathrm{Sr}$. Dive 1363 basalt glasses are similar to basalts from SEMFR erupted near the trench and to basalts of the Mariana Trough backarc basin, and these melts may be derived from similar hydrous asthenosphere that underlies the southern Mariana backarc basin and forearc. Basalt fragments and palagonitized matrix dominate the three samples we studied, but small xenocrysts and xenoliths derived from mantle peridotite and Neogene volcanics are also present, probably torn from the vent walls. Dive 1363 hyaloclastites erupted at 3-6 km water depth accompanied by vigorous degassing of volatiles, most likely $\mathrm{CO}_{2}$. These results provide further evidence that the forearc adjacent to the Challenger Deep has been invaded by asthenospheric mantle and is unusually weak. Thin lithosphere of this region may result in weak coupling between the subducting Pacific plate and overriding Mariana plate, which may be partly responsible for the great depth of the Challenger Deep.

\section{ACKNOWLEDGEMENTS}


Funding for this research includes NSF grant OCE-0961811 to Martinez; NSF OCE$\underline{0961559}$ and NSF EAR- 1258940 to Kelley, and NSF-OCE 0961352 to Stern. We gratefully acknowledge the support of the crews of R/V Yokosuka and DSV Shinkai, R/V Thomas G. Thompson and the Hawaii Mapping Research Group without whom this research would have been impossible.

\section{REFERENCES}

ARMSTRONG A. A. 2011. Cruise Report USNS Sumner, U.S. Extended Continental Shelf Cruise to Map Sections of the Mariana Trench and the Eastern and Southern Insular Margins of Guam and the Northern Mariana Islands, http://ccom.unh.edu/publications/Armstrong_2011_cruise_report_SU1002_Marianas.pdf.

BECKER N. C., FRYER P., \& MOORE G. F. 2010. Malaguana-Gadao Ridge: Identification and implications of a magma chamber reflector in the southern Mariana Trough, Geochemistry Geophysics, Geosystems 11(4), Q04X13.

BOYNTON W. V., 1985. Chapter 3. Cosmochemistry of the Rare Earth Elements: Meteorite studies, In Henderson, P. (ed.), Rare Earth Element Geochemistry Developments in Geochemistry Vol. 2, pp. 115-1522, Elsevier, Amsterdam.

BROUNCE M., KELLEY K.A., COTTRELL E., in revision. Variations in $\mathrm{Fe}^{3+} / \sum \mathrm{Fe}$ ratios of Mariana Arc basalts and primary $\mathrm{fO}_{2}$ of the mantle wedge. Journal of Petrology 
CONDIE K.C. 2001. Mantle Plumes and their Record in Earth History. Cambridge U. Press, 306 p.

EABY-DIXON J., \& STOLPER E. 1995. An Experimental Study of Water and Carbon Dioxide Solubilities in Mid-Ocean Ridge Basaltic Liquids. Part II: Applications to Degassing. Journal of Petrology 36, 1633-1646.

FISHER R.V. 1984. Submarine volcaniclastic rocks. In Kokelaar B.P. \& HOWELLS M.F. (eds.) Marginal Basin Geology: Volcanic and Associated Sedimentary and Tectonic Processes in Modern and Ancient Marginal Basins. Geological Society, London, Special Publication Vol. 16, pp. 5-27.

FRYER P., BECKER N., APPLEGATE B., MARTINEZ F., EDWARDS, M. \& FRYER G. 2003. Why is the Challenger Deep so deep? Earth and Planetary Science Letters 211, 259-269.

GALE A., DALTON C.A., LANGMUIR C.H., SU Y. \& SCHILLING J.-G. 2013. The mean composition of ocean ridge basalts. Geochemistry, Geophysics, Geosystems 14, doi:10.1029/2012GC004334.

GOVERS R., \& WORTEL M. J. R. 2005. Lithosphere tearing at STEP faults: Response to edges of subduction zones. Earth and Planetary Science Letters 236, 505-523. 
GVIRTZMAN Z. \& STERN R.J. 2004. Bathymetry of Mariana Trench-Arc System and Formation of the Challenger Deep as a Consequence of Weak Plate Coupling. Tectonics TC2011, doi:10.1029/2003TC001581

HOFMANN A.W. 1988. Chemical differentiation of the Earth: the relationship between mantle, continental crust, and oceanic crust. Earth and Planetary Science Letters 90, 297314.

KELLEY K.A. \& COTTRELL E. 2012. The influence of magmatic differentiation on the oxidation state of Fe in a basaltic arc magma. Earth and Planetary Science Letters 329330, 109-121.

KELLEY K. A., PLANK T., GROVE T.L., STOLPER E.M., NEWMAN S. \& HAURI E. 2006. Mantle melting as a function of water content beneath back-arc basins. Journal of Geophysical Research: Solid Earth 111, doi:10.1029/2005JB003732.

KITADA K., SEAMA N., YAMAZAKI T., NOGI Y., \& SUYEHIRO K. 2006. Distinct regional differences in crustal thickness along the axis of the Mariana Trough, inferred from gravity anomalies. Geochemistry, Geophysics, Geosystems 7 (4)

DOI: $10.1029 / 2005 \mathrm{GC} 001119$

LYTLE M.L., KELLEY K.A., HAURI E.H., GILL J.B., PAPIA D., ARCULUS R.J., 2012. 
Tracing mantle sources and Samoan influence in the northwestern Lau back-arc basin.

Geochemistry, Geophysics, Geosystems 13, doi:10.1029/2012gc004233.

MARTINEZ F., FRYER P. \& BECKER N. 2000. Geophysical characteristics of the southern Mariana Trough, $1^{\circ} 50^{\prime} \mathrm{N}-13^{\circ} 40^{\prime} \mathrm{N}$, Journal of Geophysical Research: Solid Earth 105(B7), $16591-16607$.

MARTINEZ F., KELLEY K. A. and STERN R. J. 2012. Creation and Deformation of Hydrous Lithosphere at the Southern Mariana Margin, EGU General Assembly 2012, Abstract EGU20126643

MATSUDA T. \& UYEDA S. 1971. On the Pacific type orogeny and its model extension of the paired belts concept and possible origin of marginal seas. Tectonophysics 11, 5-27.

NEWMAN S. \& LOWENSTERN J.B. 2002. VolatileCalc: a silicate melt- $\mathrm{H}_{2} \mathrm{O}-\mathrm{CO}_{2}$ solution model written in Visual Basic for Excel. Computers \& Geosciences 28, 597-604.

OHARA Y., REAGAN M.K., FUJIKURA K., WATANABE H., MICHIBAYASHI K., ISHII T., STERN R.J., PUJANA I., MARTINEZ F., GIRAUD G., RIBEIRO J., BROUNCE M., KOMORI N. \& KINO M. 2012. A serpentinite-hosted ecosystem in the Southern Mariana Forearc. Proceedings of the National Academy of Science Www.pnas.org/cgi/doi/10.1073/pnas.1112005109 
PEARCE J. A. 2008. Geochemical fingerprinting of oceanic basalts with applications to ophiolite classification and the search for Archean oceanic crust. Lithos 100, 14-48.

PLANK T. 2005. Constraints from Thorium/Lanthanum on Sediment Recycling at Subduction Zones and the Evolution of the Continents. Journal of Petrology 46, 921-944.

RESING, J.L. \& 23 others. 2011. Active submarine eruption of boninite in the northeastern Lau Basin. Nature Geoscience 4, 799-806.

RIBEIRO J., STERN R. J., KELLEY K., MARTINEZ F., ISHIZUKA O. \& MANTON W. I. 2013. Nature and distribution of slab-derived fluids and mantle sources beneath the SE Mariana Forearc Rift. Geochemistry, Geophysics, Geosystems 14, doi: 10.1002/ggge.20244

SANDWELL D.T. \& SMITH W.H.F. 1997. Marine gravity anomaly from Geosat and ERS 1 satellite altimetry. Journal of Geophysical Research (Solid Earth) 192, 1003910054.

SHERVAIS J. W. 1982. Ti-V plots and the petrogenesis of modern and ophiolitic lavas. Earth and Planetary Science Letters 59, 101-118. 
STERN R.J., TAMURA Y., MASUDA H., FRYER P., MARTINEZ F., ISHIZUKA O., \& BLOOMER S.H., 2013. How does the Mariana Arc end in the south? The Island Arc 22, 133-148.

SUN S. - S. \& McDONOUGH W.F., 1989. Chemical and isotopic systematics of oceanic basalts: Implications for mantle composition and processes. In Saunders A.D. \& Norry M.J. (eds.) Magmatism in the Ocean Basins, Geological Society of London, Special Publication 42, pp. 313-345. 
Fig. 1. Location of the study area. (a) Bathymetric map of the Mariana convergent margin in the Western Pacific, including Mariana Trench, Mariana Arc, and Mariana Trough (back-arc basin). Map was compiled from available bathymetric data including decimated one-minute grid for Mariana Trough (Kitada et al., 2006).. Swath-mapped bathymetry is recompiled and matched to predicted bathymetry from Sandwell and Smith (1997). Red line is back-arc basin spreading axis from Martinez and Taylor (2003). Islands are black, the largest and southernmost is Guam, USA. Dashed box outlines region shown in (b). (b) Bathymetry of the southern Mariana Trough, showing location of spreading ridge (inflated Malaguana-Gadao Ridge (MGR) in the south, axial rift of Mariana Trough spreading ridge (MTSR) farther north) and Fina Nagu Volcanic Chain (FNVC), also West Santa Rosa Bank Fault (WSRBF), Alphabet Seamount Volcanic Province (AVSP), Southern Mariana Forearc Ridge (S Mar FA Rg), Southeast Mariana Forearc Rift (SEMFR), Challenger Deep (CD), and Shinkai Seep (SS). The active magmatic arc southwest of Tracey Seamount is poorly constrained due to the unknown age of arc-like features (e.g. FNVC), but active volcanism is present from Tracey Seamount in the northeast to Toto caldera. Most data are from 'Law of the Sea' mapping carried out by the University of New Hampshire group (Armstrong, 2010) with additional data from US-NGDC and JAMSTEC databases. Small dashed box shows study area in Fig. 2, large dashed box shows HMR-1 sonar image in Fig. 7. Possible eruption sites are marked with '*'.

Fig. 2: A) Detailed bathymetric map showing bathymetry around YK1308 Shinkai dives 1363 and 1364 and location of Shinkai Seep (SSP) (Ohara et al., 2012). B) IMI30 sidescan sonar image of region. High backscatter shown with darker shading. C) 
Photograph from Shinkai 6500 submersible showing typical seafloor observed during Dive 1363. Fragments and cobbles on seafloor are dominated by basaltic volcaniclastics like those studied here.

Fig. 3: Hand specimen of YK1308 dive 1363 R15 volcaniclastic sediment, entire section (A) and close-up (B). Note that most of the visible fragments appear to be glassy basalt fragments.

Fig. 4: Electron backscatter images of millimeter-sized expanses of volcaniclastic matrix, with chemical analyses by EMP of selected fragments (minerals and glass) listed to the right of each image. Delicate fragments suggesting massive debris flow is shown by yellow 'D'. A: R06, with fragments of hornblende (a), bytownite (b), clinopyroxene (c), and glass (d). B: R15, with fragments of plagioclase (1), serpentinite (2, a), glass (b), and olivine (c). C: R15, with fragments of olivine (3, a), serpentinite (7 d), clinopyroxene, (b), amphibole (c), plagioclase (e), and glass (f).

Fig. 5: Chondrite-normalized REEs pattern for three DIVE 1363 glasses listed in Table 3. Mean compositions of "All MORB” (Gale et al., 2013) and mean SEMFR basalt (Ribeiro et al., 2013b) are also plotted for comparison. Concentrations are normalized to chondrite abundances of Boynton 1985).

Fig. 6. Extended trace element diagram for 1363 glasses compared with patterns for 'Mean SE-SEMFR' (Ribeiro et al. 2013d) and "All-MORB”' (Gale et al. 2013). Grey 
field is Mariana Trough BABB from Brounce et al. (in press). Normalizing abundances and element order from Sun and McDonough (1989).

Fig. 7: HMR-1 sonar backscatter imagery (see Fryer et al., 2003 for data description) over part of southern Mariana arc (location shown in Fig. 1b). White box shows location of Fig. 2; Possible eruption sites are marked with '*', also shown in Fig. 1b; “CD” marks location of Challenger Deep. Dark areas correspond to steep or bare-rock surfaces, light areas are flat and sediment-covered. Ship tracks trend approximately E-W and regions directly beneath ship are poorly imaged (pixelated swaths). Toothed line shows approximate trace of the Mariana Trench. 

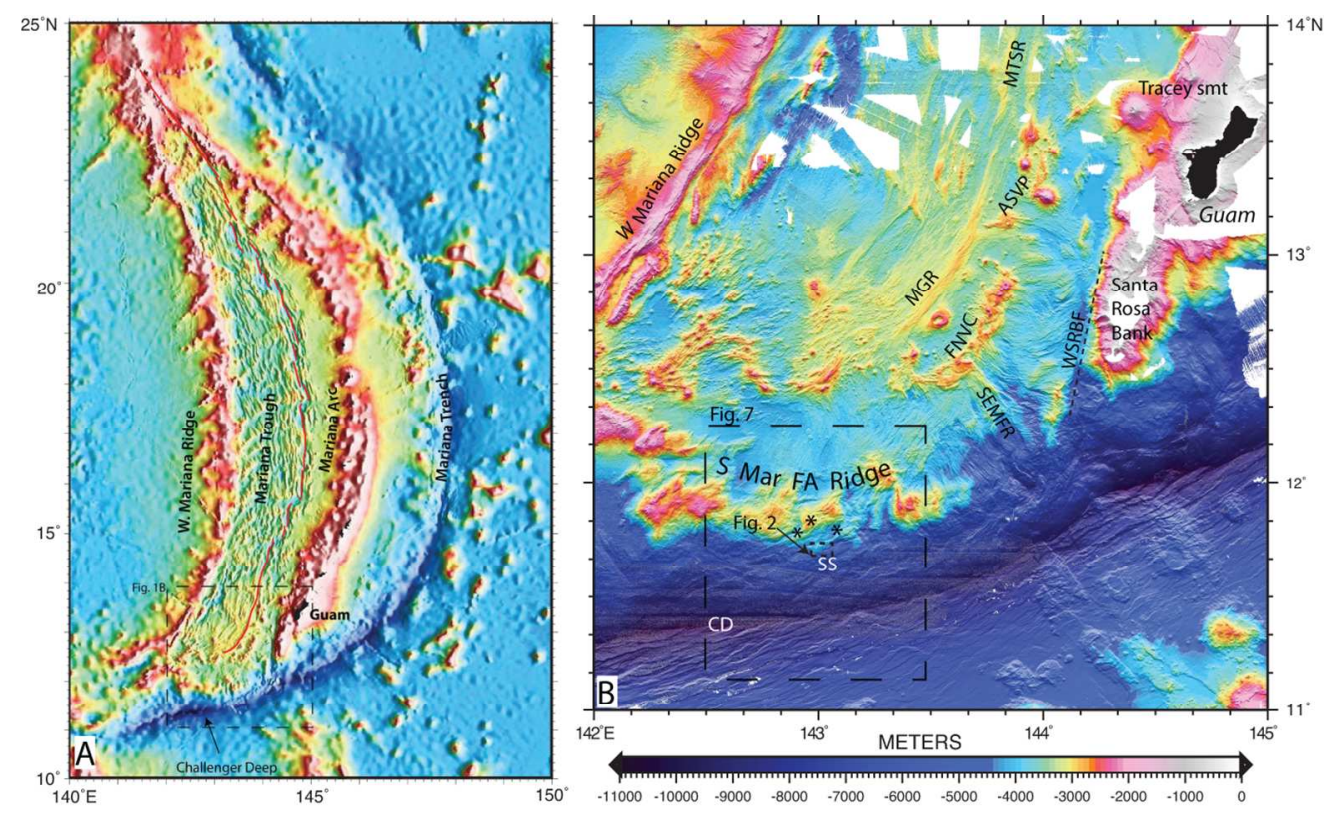

Fig. 1

Fig. 1

$177 \times 169 \mathrm{~mm}(200 \times 200$ DPI $)$ 

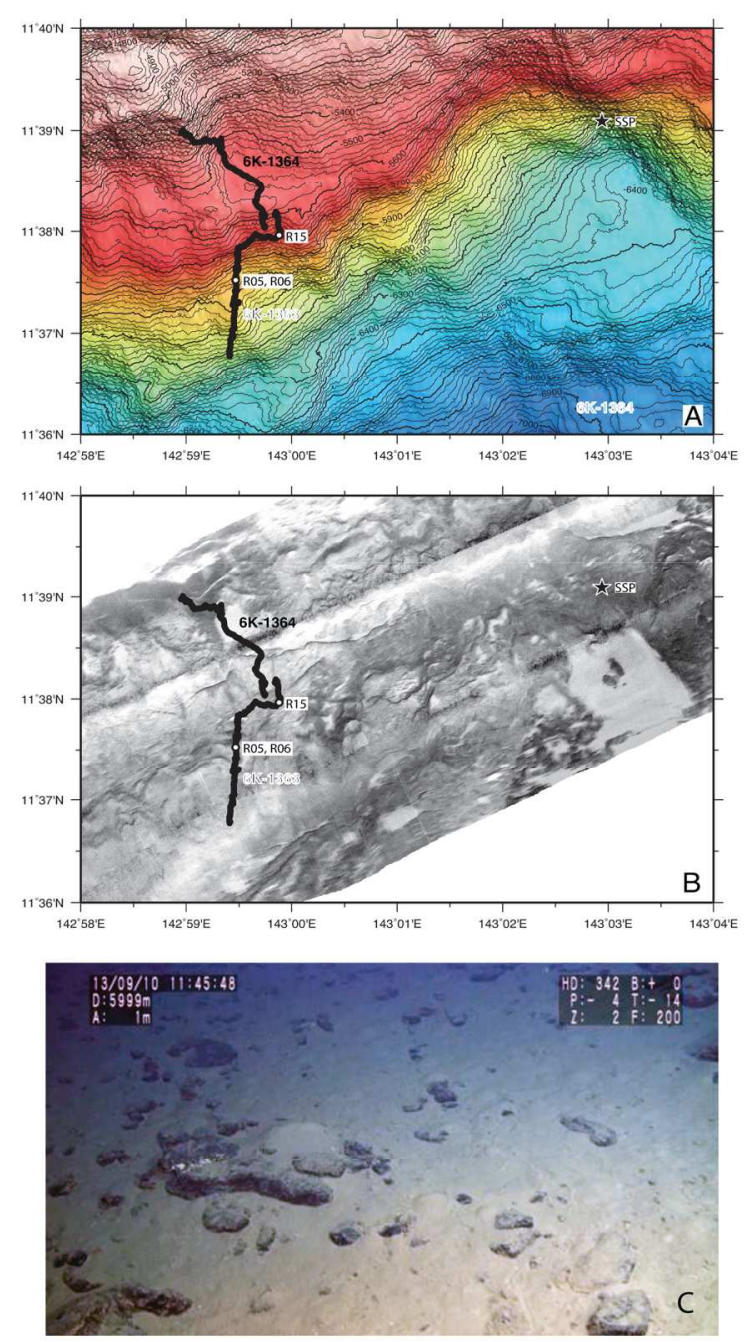

Figure 2

Fig. 2

$141 \times 255 \mathrm{~mm}(220 \times 220$ DPI $)$ 

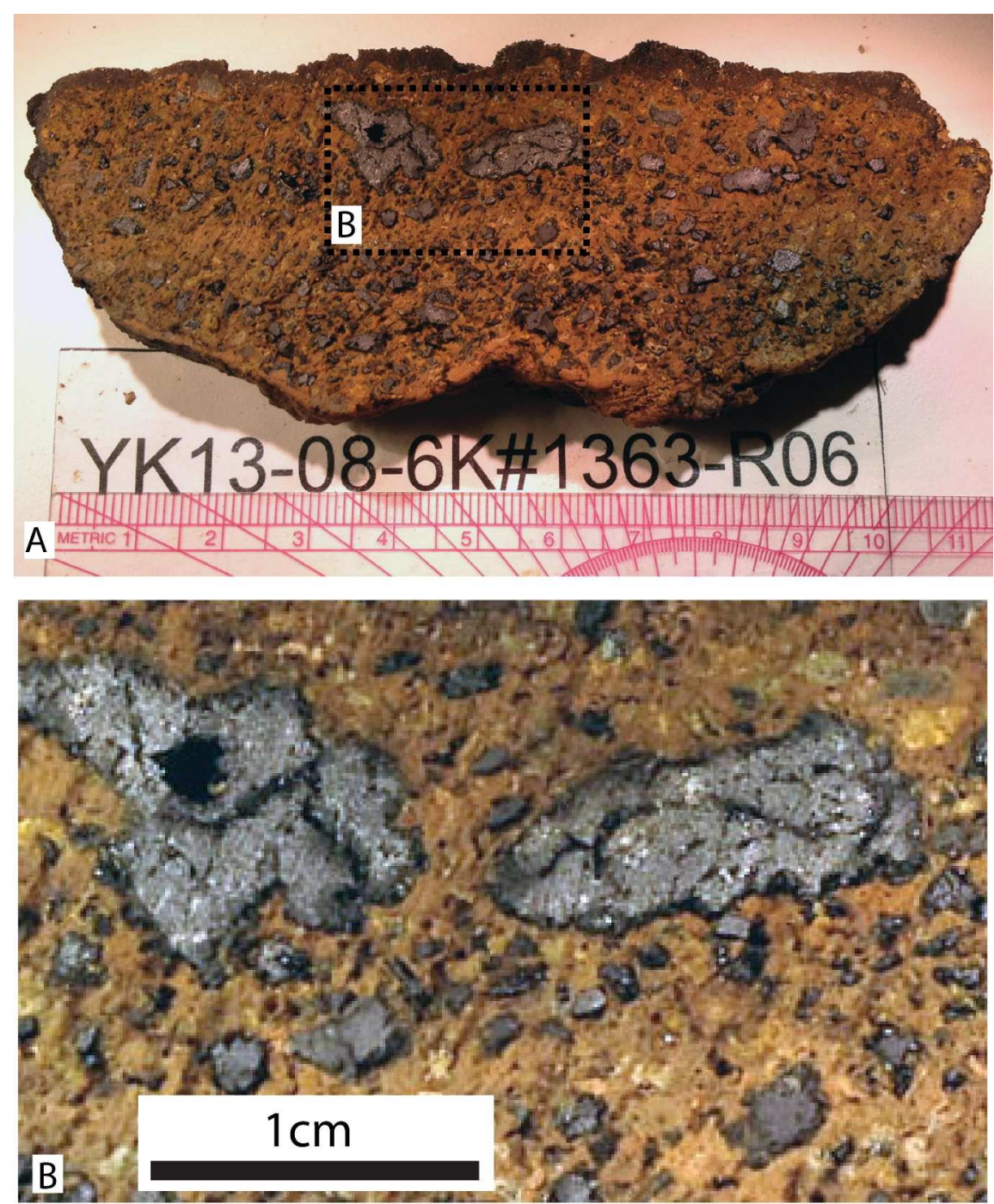

Fig. 3

$225 \times 308 \mathrm{~mm}(300 \times 300$ DPI $)$ 

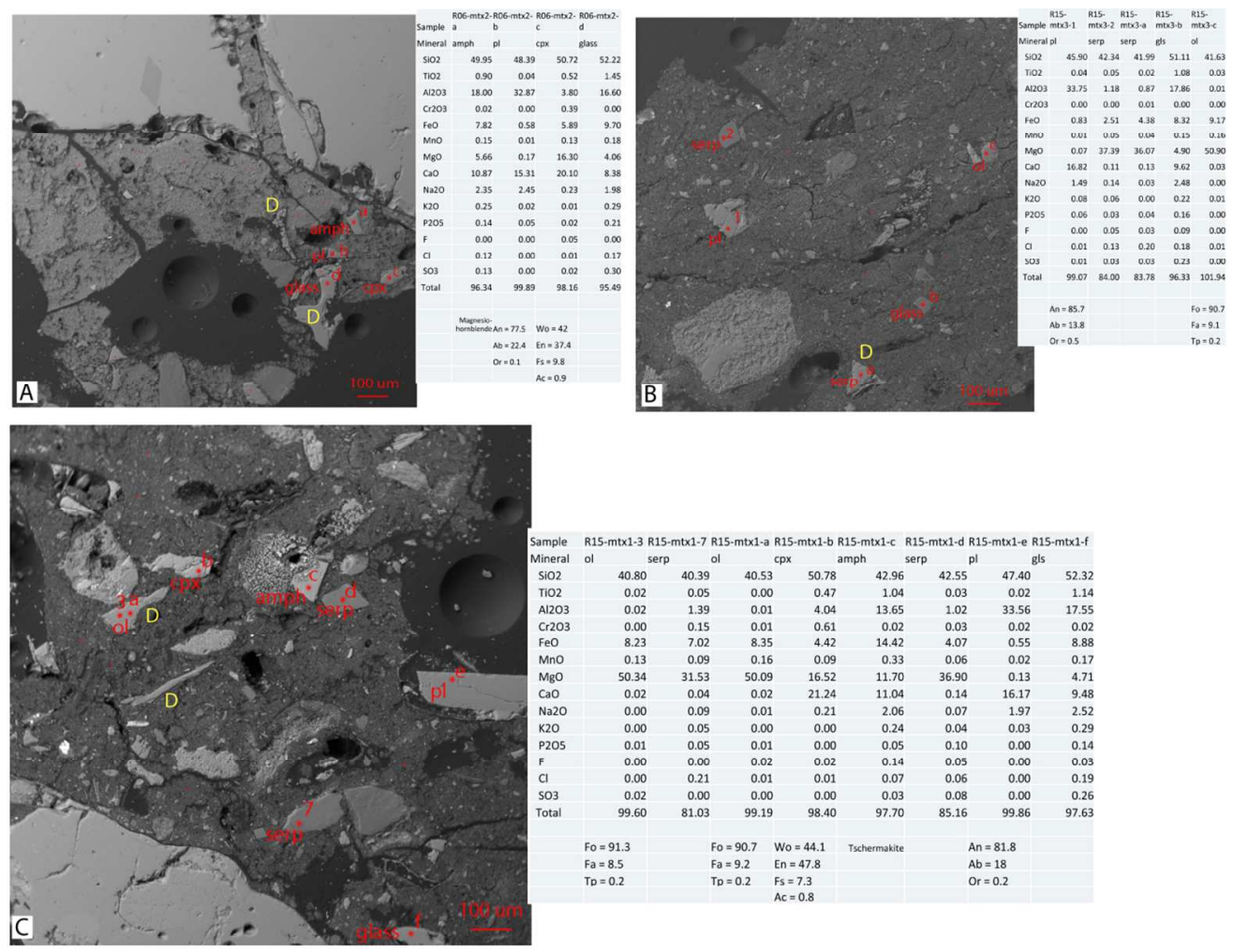

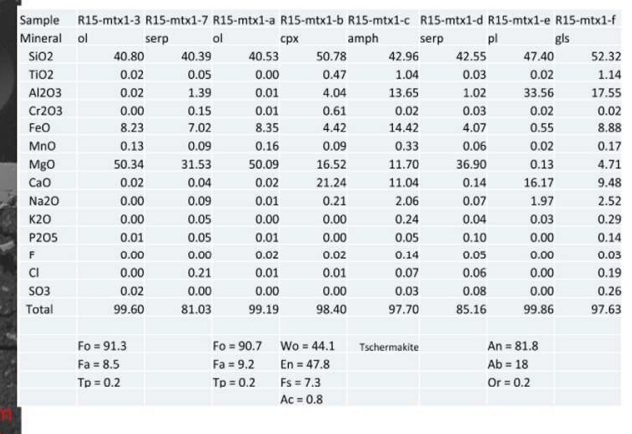

Fig. 4

$177 \times 163 \mathrm{~mm}(200 \times 200$ DPI $)$ 


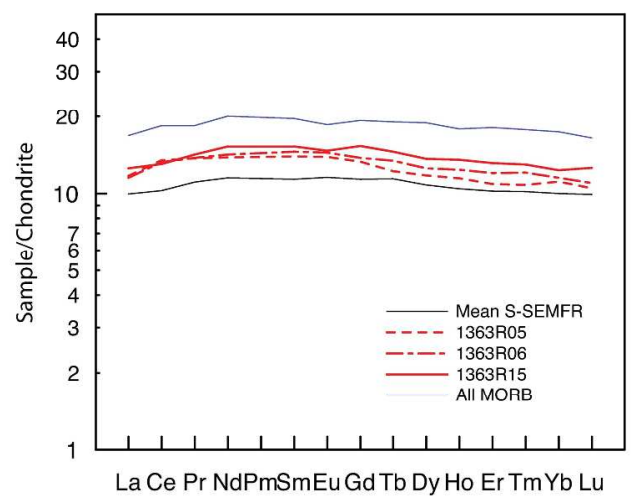

Fig. 5

$279 \times 361 \mathrm{~mm}(300 \times 300$ DPI $)$

Island Arc, For Peer Review 


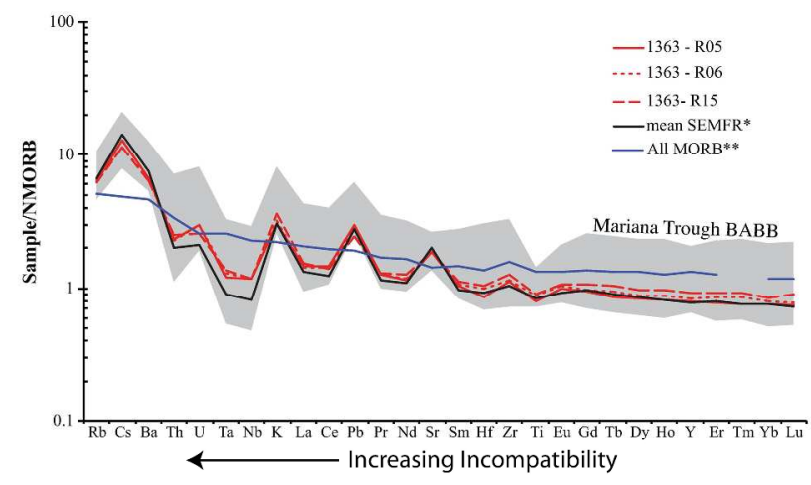

Fig. 6

Fig. 6

$279 \times 361 \mathrm{~mm}(300 \times 300$ DPI $)$ 


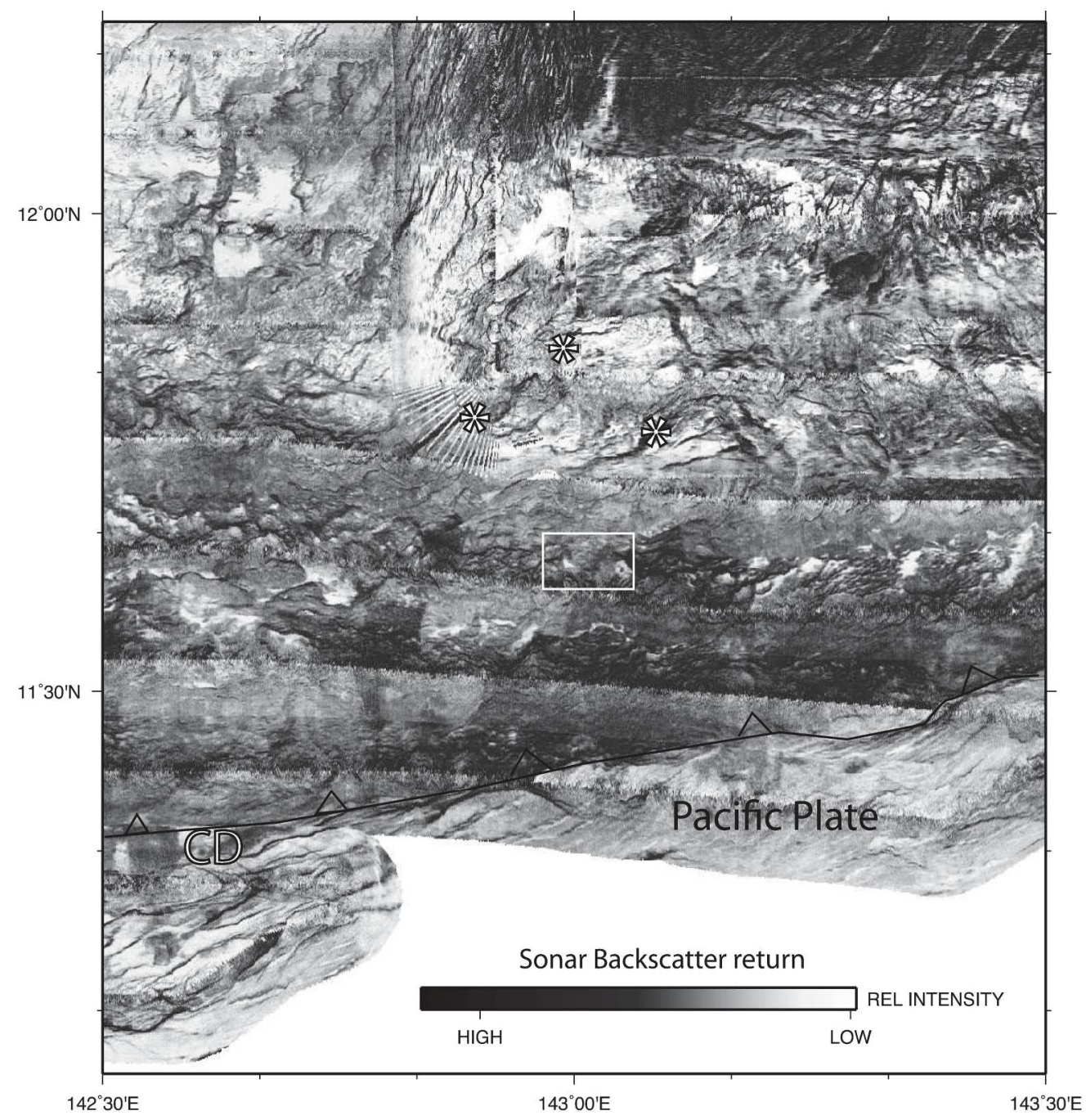

Fig. 7

Fig. 7

$214 \times 246 \mathrm{~mm}(300 \times 300$ DPI $)$ 
Table 1: Major Element Analyses of YK1308 Dive 1363 basaltic glass fragments

\begin{tabular}{|c|c|c|c|c|c|c|c|}
\hline \multirow[b]{3}{*}{$\mathrm{N}$} & \multicolumn{2}{|c|}{ R05 } & \multicolumn{2}{|c|}{ R06 } & \multicolumn{2}{|c|}{ R15 } & \multirow{2}{*}{$\begin{array}{l}\text { ¿E-SEMFR M } \\
\text { Mean }\end{array}$} \\
\hline & Mean & stdev & Mean & tdev & Mean & tdev & \\
\hline & 153 & & 131 & & 145 & & 6 \\
\hline $\mathrm{SiO} 2$ & 51.00 & 0.39 & 51.37 & 0.42 & 51.94 & 0.25 & 51.48 \\
\hline TiO2 & 1.01 & 0.07 & 1.10 & 0.06 & 1.14 & 0.05 & 1.03 \\
\hline $\mathrm{Al} 2 \mathrm{O} 3$ & 16.38 & 0.27 & 16.38 & 0.28 & 16.46 & 0.64 & 15.73 \\
\hline $\mathrm{Cr} 2 \mathrm{O} 3$ & 0.01 & 0.01 & 0.01 & 0.01 & 0.01 & 0.01 & \\
\hline $\mathrm{FeO}^{*}$ & 8.23 & 0.31 & 8.66 & 0.25 & 8.85 & 0.34 & 8.53 \\
\hline $\mathrm{MnO}$ & 0.15 & 0.02 & 0.16 & 0.02 & 0.16 & 0.02 & 0.15 \\
\hline $\mathrm{MgO}$ & 6.17 & 0.52 & 5.59 & 0.64 & 5.36 & 1.28 & 5.62 \\
\hline $\mathrm{CaO}$ & 11.01 & 0.56 & 10.34 & 0.68 & 10.08 & 0.27 & 10.05 \\
\hline $\mathrm{Na} 2 \mathrm{O}$ & 2.88 & 0.19 & 3.07 & 0.22 & 3.20 & 0.10 & 3.22 \\
\hline $\mathrm{K} 2 \mathrm{O}$ & 0.22 & 0.02 & 0.23 & 0.04 & 0.26 & 0.01 & 0.25 \\
\hline P2O5 & 0.11 & 0.02 & 0.12 & 0.03 & 0.12 & 0.03 & 0.11 \\
\hline $\mathrm{F}$ & 0.03 & 0.04 & 0.02 & 0.04 & 0.03 & 0.05 & 0.02 \\
\hline $\mathrm{Cl}$ & 0.10 & 0.02 & 0.13 & 0.03 & 0.13 & 0.01 & 0.04 \\
\hline SO3 & 0.19 & 0.02 & 0.28 & 0.18 & 0.20 & 0.02 & 0.22 \\
\hline Total & 97.49 & & 97.46 & & 97.94 & & 96.45 \\
\hline $\mathrm{H} 2 \mathrm{O}$ (wt. \%) & 1.97 & 0.05 & 2.29 & & 2.06 & 0.16 & $2.04 * * *$ \\
\hline $\mathrm{CO} 2(\mathrm{ppm})$ & 455 & 116 & 539 & & 94 & 22 & 51 \\
\hline $\mathrm{Mg} \#$ & 57.2 & & 53.5 & & 51.9 & & 54.0 \\
\hline \multicolumn{8}{|c|}{ CIPW NORMS $* * * * *$} \\
\hline QZ & 1.69 & & 2.52 & & 2.1 & & 2.56 \\
\hline $\mathrm{PL}$ & 57 & & 57.82 & & 58.77 & & 57.03 \\
\hline OR & 1.36 & & 1.42 & & 1.6 & & 1.54 \\
\hline DI & 19.1 & & 17.1 & & 16.59 & & 18.18 \\
\hline HY & 15.9 & & 15.85 & & 17.47 & & 15.53 \\
\hline \multicolumn{8}{|l|}{ OL } \\
\hline IL & 1.98 & & 2.15 & & 0.27 & & 2.03 \\
\hline MT & 2.73 & & 2.87 & & 2.94 & & 2.86 \\
\hline$A P$ & 0.25 & & 0.28 & & 0.28 & & 0.25 \\
\hline
\end{tabular}

$* *$ wt. $\%$, calculated volatile free, Fe $3+/$ total $=0.2 ;$ adjusted to total 1( $* * *$ Ribeiro et al., 2013b; volatiles from Ribeiro et al. submitted $* * * *$ Gale et al. 2013

$* * * * *$ Michael 1995 


\begin{tabular}{|c|c|c|}
\hline \multirow{2}{*}{$\begin{array}{l}\text { ean Glass**: } \\
\text { stdev }\end{array}$} & \multicolumn{2}{|c|}{ ALL MORB $* * * *$} \\
\hline & 430 & \\
\hline 0.91 & 50.47 & 0.08 \\
\hline 0.19 & 1.68 & 0.05 \\
\hline 0.27 & 14.70 & 0.12 \\
\hline 0.70 & 10.43 & 0.21 \\
\hline 0.02 & 0.18 & 0.01 \\
\hline 0.86 & 7.58 & 0.12 \\
\hline 1.08 & 11.39 & 0.09 \\
\hline 0.46 & 2.79 & 0.03 \\
\hline 0.05 & 0.16 & 0.01 \\
\hline 0.03 & 0.18 & 0.01 \\
\hline
\end{tabular}

99.56

$0.15^{* * * *}$

56.4

50.93

0.95

23.36

12.61

8.52

3.21

0.42 
Table 2: Trace Element contents of YK1308 Dive 1363 basaltic glass

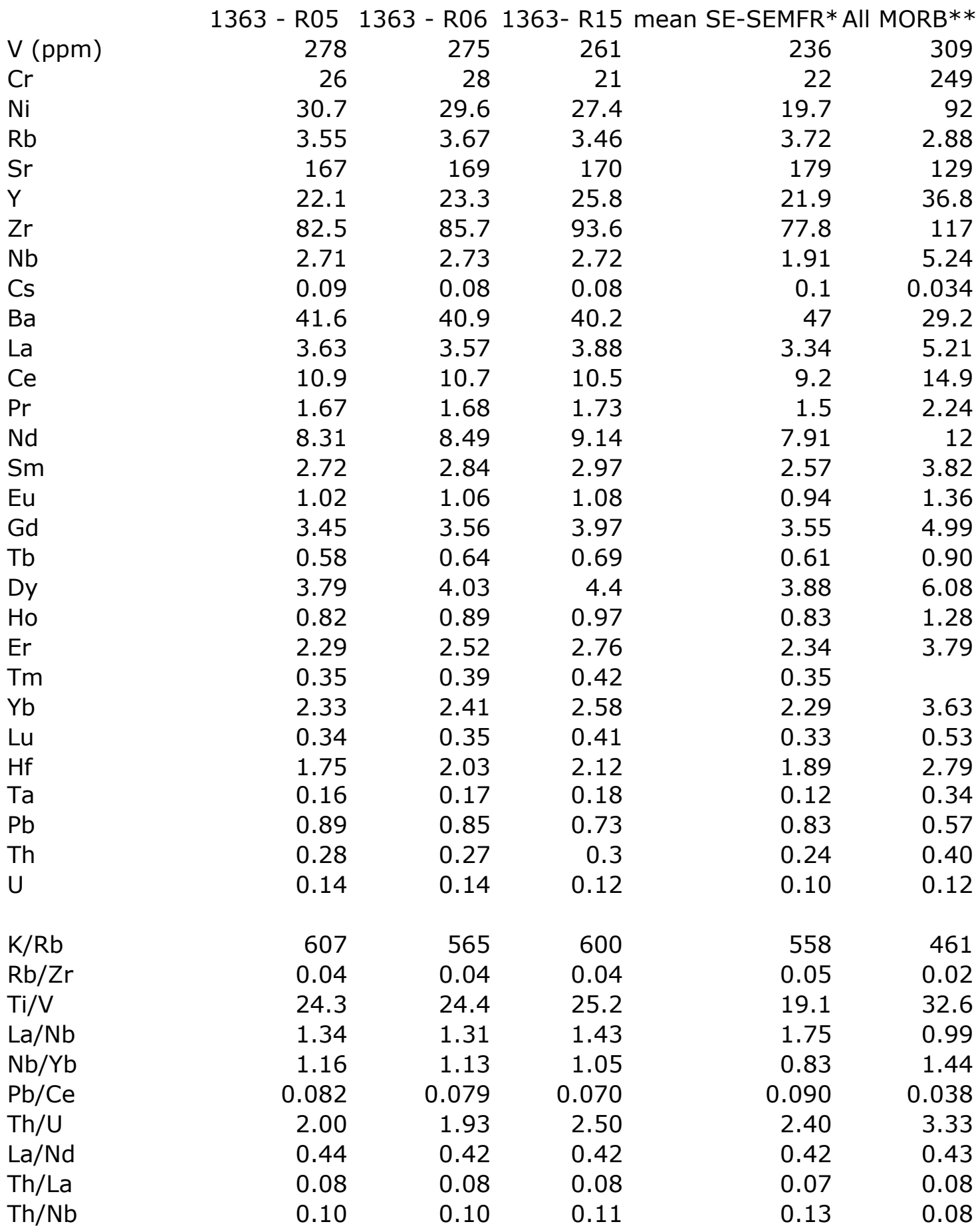

* Ribeiro et al. 2013

**Gale et al. 2013 
Supplementary Document 2. Electron microprobe analyses for minerals in glass

clinopyroxene

\begin{tabular}{|c|c|c|c|c|c|c|c|c|c|}
\hline R05-cpx & $\mathrm{SiO} 2$ & TiO2 & $\mathrm{Al} 2 \mathrm{O} 3$ & $\mathrm{Cr} 2 \mathrm{O} 3$ & $\mathrm{FeO}$ & $\mathrm{MnO}$ & $\mathrm{MgO}$ & $\mathrm{CaO}$ & $\mathrm{Na} 2 \mathrm{O}$ \\
\hline R05-18 & 52.59 & 0.56 & 2.74 & 0.31 & 4.88 & 0.13 & 16.22 & 21.98 & 0.28 \\
\hline R05-18 & 52.49 & 0.54 & 2.77 & 0.31 & 5.16 & 0.14 & 16.33 & 21.98 & 0.27 \\
\hline R05-18 & 52.58 & 0.55 & 2.59 & 0.32 & 4.94 & 0.10 & 16.28 & 22.28 & 0.29 \\
\hline R05-18 & 52.61 & 0.58 & 2.60 & 0.31 & 4.80 & 0.12 & 16.34 & 22.46 & 0.30 \\
\hline R05-18 & 52.60 & 0.59 & 2.72 & 0.34 & 4.93 & 0.13 & 16.32 & 22.15 & 0.30 \\
\hline R05-18 & 52.75 & 0.55 & 2.54 & 0.29 & 5.06 & 0.12 & 16.09 & 21.90 & 0.30 \\
\hline R05-18 & 52.63 & 0.53 & 2.53 & 0.30 & 5.10 & 0.10 & 16.27 & 22.11 & 0.28 \\
\hline R05-18 & 52.67 & 0.51 & 2.61 & 0.29 & 5.06 & 0.11 & 16.31 & 22.03 & 0.26 \\
\hline R05-18 & 51.36 & 0.64 & 3.78 & 0.23 & 5.94 & 0.15 & 15.91 & 21.48 & 0.20 \\
\hline R05-18 & 51.70 & 0.60 & 3.73 & 0.20 & 5.93 & 0.16 & 16.12 & 21.07 & 0.22 \\
\hline R05-18 & 51.83 & 0.56 & 3.33 & 0.24 & 5.86 & 0.17 & 16.32 & 21.17 & 0.23 \\
\hline R05-18 & 52.06 & 0.58 & 2.74 & 0.33 & 4.88 & 0.08 & 16.14 & 22.38 & 0.27 \\
\hline R05-18 & 52.15 & 0.57 & 3.27 & 0.28 & 5.84 & 0.13 & 16.39 & 21.20 & 0.25 \\
\hline R05-18 & 52.19 & 0.49 & 3.17 & 0.33 & 5.72 & 0.14 & 16.45 & 21.19 & 0.23 \\
\hline R05-cpx1 & 50.97 & 0.65 & 4.09 & 0.24 & 6.39 & 0.14 & 16.19 & 20.28 & 0.23 \\
\hline R05-cpx2 & 50.23 & 1.03 & 5.46 & 0.10 & 6.87 & 0.17 & 15.19 & 20.14 & 0.28 \\
\hline R05-cpx2 & 53.46 & 0.38 & 2.19 & 0.14 & 6.48 & 0.20 & 18.07 & 19.18 & 0.18 \\
\hline R05-срx2 & 51.14 & 0.74 & 4.47 & 0.28 & 6.10 & 0.11 & 15.44 & 21.22 & 0.27 \\
\hline R05-cpx2 & 53.37 & 0.41 & 2.27 & 0.15 & 6.95 & 0.21 & 18.51 & 18.18 & 0.15 \\
\hline
\end{tabular}

\begin{tabular}{lrrrrrrrrr} 
R06-cpx & \multicolumn{1}{l}{$\mathrm{SiO2}$} & $\mathrm{TiO} 2$ & $\mathrm{Al} 2 \mathrm{O} 3$ & $\mathrm{Cr} 2 \mathrm{O} 3$ & $\mathrm{FeO}$ & $\mathrm{MnO}$ & $\mathrm{MgO}$ & $\mathrm{CaO}$ & $\mathrm{Na} 2 \mathrm{O}$ \\
R06-14 & 51.65 & 0.60 & 4.08 & 0.48 & 6.19 & 0.13 & 16.61 & 20.36 & 0.23 \\
R06-14 & 53.43 & 0.33 & 2.19 & 0.15 & 6.53 & 0.18 & 18.44 & 18.80 & 0.20 \\
R06-13 & 51.36 & 0.66 & 6.92 & 0.45 & 5.44 & 0.12 & 14.35 & 19.43 & 0.74 \\
R6-14-2 & 51.46 & 0.74 & 4.15 & 0.32 & 6.17 & 0.14 & 15.72 & 21.60 & 0.26 \\
R6-14-2 & 51.66 & 0.62 & 3.92 & 0.35 & 6.06 & 0.14 & 15.63 & 21.18 & 0.24 \\
R6-14-2 & 51.10 & 0.84 & 4.63 & 0.33 & 6.20 & 0.15 & 15.63 & 21.20 & 0.24 \\
R6-14-2 & 50.51 & 0.60 & 4.73 & 0.38 & 5.98 & 0.12 & 15.84 & 21.12 & 0.29 \\
R6-14-2 & 50.84 & 0.79 & 4.67 & 0.42 & 6.00 & 0.12 & 15.55 & 21.53 & 0.28 \\
& & & & & & & & & \\
R15-cpx & SiO2 & TiO2 & Al2O3 & Cr2O3 & FeO & MnO & MgO & CaO & Na2O \\
R15-12 & 53.56 & 0.35 & 1.78 & 0.26 & 5.74 & 0.16 & 17.46 & 20.79 & 0.15 \\
R15-12 & 52.66 & 0.39 & 2.36 & 0.36 & 5.87 & 0.17 & 17.43 & 19.78 & 0.18 \\
R15-12 & 51.05 & 0.73 & 5.41 & 0.20 & 6.32 & 0.13 & 15.20 & 19.98 & 0.50 \\
R15-16 & 51.81 & 0.58 & 4.02 & 0.45 & 5.72 & 0.08 & 16.49 & 21.59 & 0.25 \\
R15-16 & 51.48 & 0.54 & 4.40 & 0.12 & 6.17 & 0.16 & 16.29 & 21.53 & 0.30 \\
R15-3 & 50.43 & 0.53 & 4.42 & 0.34 & 5.75 & 0.13 & 15.84 & 21.42 & 0.24 \\
R15-3 & 50.94 & 0.56 & 3.51 & 0.54 & 5.19 & 0.12 & 16.22 & 21.68 & 0.26 \\
R15-13 & 53.27 & 0.33 & 2.18 & 0.08 & 6.65 & 0.20 & 18.17 & 19.22 & 0.20 \\
R15-13 & 53.51 & 0.36 & 2.44 & 0.10 & 6.64 & 0.17 & 18.05 & 19.45 & 0.20 \\
R15-13 & 53.04 & 0.35 & 2.73 & 0.09 & 6.28 & 0.19 & 17.18 & 20.42 & 0.21 \\
R15-13 & 51.46 & 0.69 & 4.49 & 0.18 & 6.28 & 0.17 & 15.16 & 21.64 & 0.29 \\
R15-13 & 51.34 & 0.79 & 4.70 & 0.14 & 6.60 & 0.18 & 15.63 & 21.29 & 0.27
\end{tabular}




\begin{tabular}{|c|c|c|c|c|c|c|c|c|c|}
\hline R05-pl & $\mathrm{SiO} 2$ & TiO2 & $\mathrm{Al} 2 \mathrm{O} 3$ & $\mathrm{Cr} 2 \mathrm{O} 3$ & $\mathrm{FeO}$ & $\mathrm{MnO}$ & $\mathrm{MgO}$ & $\mathrm{CaO}$ & $\mathrm{Na} 2 \mathrm{O}$ \\
\hline R05-1 & 47.27 & 0.04 & 33.56 & 0.00 & 0.61 & 0.00 & 0.15 & 16.23 & 1.87 \\
\hline R05-1 & 48.97 & 0.04 & 32.29 & 0.01 & 0.67 & 0.01 & 0.17 & 15.00 & 2.74 \\
\hline R05-1 & 49.61 & 0.03 & 31.65 & 0.00 & 0.66 & 0.02 & 0.17 & 14.41 & 3.04 \\
\hline R05-1 & 49.42 & 0.05 & 31.91 & 0.02 & 0.70 & 0.05 & 0.16 & 14.41 & 3.02 \\
\hline R05-2 & 47.91 & 0.00 & 32.73 & 0.02 & 0.50 & 0.01 & 0.20 & 15.64 & 2.38 \\
\hline R05-2 & 50.03 & 0.06 & 31.16 & 0.00 & 0.50 & 0.01 & 0.20 & 13.83 & 3.07 \\
\hline R05-2 & 47.95 & 0.00 & 32.64 & 0.00 & 0.51 & 0.02 & 0.16 & 15.60 & 2.36 \\
\hline R05-2 & 47.98 & 0.03 & 32.68 & 0.00 & 0.52 & 0.01 & 0.17 & 15.47 & 2.37 \\
\hline R05-2 & 47.99 & 0.03 & 32.68 & 0.00 & 0.56 & 0.00 & 0.18 & 15.49 & 2.29 \\
\hline R05-2 & 48.94 & 0.04 & 31.85 & 0.01 & 0.59 & 0.04 & 0.20 & 14.73 & 2.90 \\
\hline R05-2 & 48.98 & 0.05 & 31.64 & 0.00 & 0.62 & 0.00 & 0.21 & 14.80 & 2.58 \\
\hline R05-3 & 49.11 & 0.07 & 31.52 & 0.01 & 0.64 & 0.00 & 0.17 & 14.20 & 2.69 \\
\hline R05-3 & 49.79 & 0.07 & 31.16 & 0.04 & 0.72 & 0.01 & 0.19 & 14.07 & 3.02 \\
\hline R05-3 & 48.97 & 0.01 & 31.65 & 0.01 & 0.72 & 0.02 & 0.14 & 14.81 & 2.72 \\
\hline R05-3 & 49.59 & 0.04 & 30.98 & 0.00 & 0.64 & 0.00 & 0.19 & 14.14 & 2.93 \\
\hline R05-3 & 49.23 & 0.02 & 31.65 & 0.01 & 0.67 & 0.00 & 0.13 & 14.43 & 2.88 \\
\hline R05-3 & 49.27 & 0.01 & 31.84 & 0.00 & 0.64 & 0.01 & 0.13 & 14.64 & 2.88 \\
\hline R05-3 & 49.02 & 0.06 & 31.81 & 0.00 & 0.71 & 0.00 & 0.15 & 14.69 & 2.71 \\
\hline R05-3 & 48.81 & 0.02 & 32.13 & 0.00 & 0.63 & 0.00 & 0.15 & 14.75 & 2.73 \\
\hline R05-3 & 48.34 & 0.00 & 32.18 & 0.00 & 0.75 & 0.01 & 0.16 & 14.48 & 2.56 \\
\hline R05-3 & 49.36 & 0.04 & 31.84 & 0.00 & 0.61 & 0.02 & 0.17 & 14.85 & 2.86 \\
\hline R05-4 & 46.89 & 0.04 & 33.38 & 0.00 & 0.60 & 0.00 & 0.20 & 16.36 & 1.92 \\
\hline R05-4 & 48.89 & 0.04 & 32.23 & 0.00 & 0.64 & 0.01 & 0.16 & 14.96 & 2.84 \\
\hline R05-4 & 50.01 & 0.04 & 30.88 & 0.00 & 0.67 & 0.01 & 0.17 & 13.48 & 3.16 \\
\hline R05-4 & 51.28 & 0.05 & 30.90 & 0.03 & 0.79 & 0.00 & 0.16 & 13.35 & 3.43 \\
\hline R05-4 & 51.26 & 0.05 & 30.84 & 0.00 & 0.80 & 0.03 & 0.16 & 13.31 & 3.61 \\
\hline R05-4 & 48.77 & 0.01 & 32.55 & 0.00 & 0.64 & 0.02 & 0.15 & 15.32 & 2.52 \\
\hline R05-4 & 49.08 & 0.02 & 31.48 & 0.00 & 0.67 & 0.00 & 0.21 & 14.72 & 2.75 \\
\hline
\end{tabular}

\begin{tabular}{lrrrrrrrrr} 
R06-pl & \multicolumn{1}{l}{$\mathrm{SiO2}$} & $\mathrm{TiO} 2$ & $\mathrm{Al} 2 \mathrm{O} 3$ & $\mathrm{Cr} 2 \mathrm{O} 3$ & $\mathrm{FeO}$ & $\mathrm{MnO}$ & $\mathrm{MgO}$ & $\mathrm{CaO}$ & $\mathrm{Na} 2 \mathrm{O}$ \\
R06-1 & 49.88 & 0.06 & 30.72 & 0.02 & 0.70 & 0.00 & 0.18 & 13.69 & 3.31 \\
R06-1 & 49.80 & 0.05 & 31.31 & 0.00 & 0.61 & 0.00 & 0.18 & 14.13 & 3.13 \\
R06-2 & 49.74 & 0.06 & 31.19 & 0.00 & 0.63 & 0.02 & 0.18 & 14.08 & 3.17 \\
R06-2 & 49.32 & 0.04 & 31.21 & 0.00 & 0.67 & 0.01 & 0.16 & 14.42 & 3.03 \\
R06-2 & 49.99 & 0.07 & 30.90 & 0.00 & 0.67 & 0.00 & 0.18 & 13.92 & 3.08 \\
R06-2 & 50.10 & 0.04 & 30.98 & 0.00 & 0.75 & 0.03 & 0.16 & 13.87 & 3.33 \\
R06-2 & 49.90 & 0.03 & 30.61 & 0.01 & 0.67 & 0.02 & 0.20 & 13.27 & 3.40 \\
R06-2 & 48.83 & 0.03 & 31.56 & 0.00 & 0.68 & 0.03 & 0.16 & 14.42 & 2.96 \\
R06-2 & 48.76 & 0.02 & 31.55 & 0.00 & 0.70 & 0.01 & 0.16 & 14.20 & 2.80 \\
R06-2 & 50.27 & 0.05 & 31.18 & 0.00 & 0.53 & 0.02 & 0.16 & 13.74 & 3.32 \\
R06-2 & 49.79 & 0.04 & 30.95 & 0.00 & 0.53 & 0.01 & 0.14 & 13.66 & 3.36 \\
R06-2 & 49.86 & 0.05 & 30.73 & 0.02 & 0.56 & 0.03 & 0.14 & 13.97 & 3.17 \\
R06-3 & 45.88 & 0.01 & 33.48 & 0.01 & 0.41 & 0.00 & 0.18 & 17.12 & 1.42 \\
R06-3 & 43.90 & 0.00 & 35.64 & 0.01 & 0.35 & 0.00 & 0.13 & 18.62 & 0.72 \\
R06-3 & 43.50 & 0.00 & 35.69 & 0.00 & 0.34 & 0.03 & 0.09 & 18.01 & 0.56
\end{tabular}




$\begin{array}{llllllllll}\text { R06-3 } & 43.96 & 0.01 & 35.62 & 0.01 & 0.33 & 0.00 & 0.09 & 18.60 & 0.57 \\ \text { R06-3 } & 43.73 & 0.01 & 35.74 & 0.00 & 0.35 & 0.00 & 0.09 & 18.67 & 0.70 \\ \text { R06-3 } & 46.66 & 0.02 & 33.66 & 0.01 & 0.50 & 0.00 & 0.18 & 16.32 & 1.86 \\ \text { R06-3 } & 46.22 & 0.02 & 33.88 & 0.02 & 0.49 & 0.00 & 0.17 & 17.16 & 1.51 \\ \text { R06-3 } & 48.69 & 0.01 & 32.15 & 0.00 & 0.54 & 0.00 & 0.20 & 14.93 & 2.57 \\ \text { R06-3 } & 48.94 & 0.07 & 32.05 & 0.00 & 0.53 & 0.00 & 0.19 & 14.76 & 2.62 \\ \text { R06-12 } & 50.96 & 0.04 & 31.41 & 0.00 & 0.59 & 0.03 & 0.22 & 14.77 & 3.21 \\ \text { R06-12 } & 50.85 & 0.02 & 31.63 & 0.00 & 0.63 & 0.00 & 0.20 & 14.48 & 3.17 \\ \text { R06-12 } & 46.40 & 0.01 & 34.55 & 0.00 & 0.45 & 0.00 & 0.16 & 17.82 & 1.32 \\ \text { R06-12 } & 46.69 & 0.01 & 34.62 & 0.00 & 0.48 & 0.01 & 0.16 & 17.99 & 1.38 \\ \text { R06-12 } & 50.31 & 0.03 & 31.65 & 0.01 & 0.60 & 0.02 & 0.24 & 15.34 & 2.95 \\ \text { R06-12 } & 50.18 & 0.00 & 31.64 & 0.00 & 0.63 & 0.00 & 0.22 & 15.41 & 2.85\end{array}$

\begin{tabular}{|c|c|c|c|c|c|c|c|c|c|}
\hline R15-pl & $\mathrm{SiO} 2$ & $\mathrm{TiO} 2$ & Al2O3 & $\mathrm{Cr} 2 \mathrm{O} 3$ & $\mathrm{FeO}$ & $\mathrm{MnO}$ & $\mathrm{MgO}$ & $\mathrm{CaO}$ & $\mathrm{Na} 2 \mathrm{O}$ \\
\hline R15-1 & 47.79 & 0.00 & 32.87 & 0.01 & 0.60 & 0.04 & 0.20 & 15.82 & 2.29 \\
\hline R15-1 & 48.66 & 0.05 & 32.65 & 0.00 & 0.59 & 0.00 & 0.21 & 15.43 & 2.51 \\
\hline R15-1 & 47.93 & 0.03 & 32.74 & 0.00 & 0.52 & 0.01 & 0.18 & 15.73 & 2.33 \\
\hline R15-1 & 48.72 & 0.05 & 31.88 & 0.01 & 0.55 & 0.01 & 0.21 & 14.89 & 2.62 \\
\hline R15-1 & 47.76 & 0.03 & 32.90 & 0.01 & 0.53 & 0.00 & 0.16 & 15.60 & 2.24 \\
\hline R15-1 & 49.53 & 0.05 & 31.67 & 0.00 & 0.66 & 0.01 & 0.15 & 14.31 & 2.98 \\
\hline R15-1 & 48.87 & 0.05 & 31.99 & 0.03 & 0.67 & 0.03 & 0.16 & 14.85 & 2.66 \\
\hline R15-1 & 48.96 & 0.06 & 31.97 & 0.00 & 0.53 & 0.01 & 0.15 & 14.70 & 2.72 \\
\hline R15-1 & 48.28 & 0.03 & 32.39 & 0.01 & 0.56 & 0.01 & 0.17 & 15.29 & 2.50 \\
\hline R15-2 & 48.47 & 0.03 & 32.13 & 0.01 & 0.62 & 0.00 & 0.16 & 15.24 & 2.53 \\
\hline R15-2 & 47.92 & 0.05 & 32.46 & 0.00 & 0.52 & 0.01 & 0.17 & 14.86 & 2.43 \\
\hline R15-2 & 46.00 & 0.00 & 33.87 & 0.01 & 0.48 & 0.00 & 0.13 & 17.07 & 1.47 \\
\hline R15-2 & 49.69 & 0.03 & 31.08 & 0.01 & 0.68 & 0.00 & 0.17 & 14.15 & 2.54 \\
\hline R15-2 & 49.76 & 0.06 & 31.48 & 0.02 & 0.67 & 0.03 & 0.17 & 13.93 & 3.05 \\
\hline R15-2 & 50.32 & 0.08 & 30.24 & 0.00 & 0.64 & 0.02 & 0.15 & 13.46 & 3.53 \\
\hline R15-2 & 49.15 & 0.04 & 31.64 & 0.00 & 0.62 & 0.02 & 0.18 & 14.38 & 2.79 \\
\hline R15-3 & 49.83 & 0.02 & 30.83 & 0.01 & 0.60 & 0.03 & 0.16 & 13.96 & 3.16 \\
\hline R15-3 & 49.86 & 0.05 & 31.42 & 0.00 & 0.61 & 0.00 & 0.18 & 14.08 & 3.12 \\
\hline R15-3 & 50.30 & 0.03 & 31.11 & 0.00 & 0.63 & 0.01 & 0.15 & 13.43 & 3.39 \\
\hline R15-3 & 50.18 & 0.07 & 30.93 & 0.01 & 0.77 & 0.01 & 0.17 & 13.70 & 3.39 \\
\hline R15-3 & 45.86 & 0.04 & 34.23 & 0.00 & 0.57 & 0.00 & 0.13 & 16.79 & 1.64 \\
\hline R15-3 & 45.77 & 0.03 & 34.17 & 0.01 & 0.58 & 0.00 & 0.11 & 17.32 & 1.36 \\
\hline R15-3 & 49.58 & 0.33 & 28.45 & 0.00 & 2.43 & 0.06 & 1.36 & 13.24 & 2.60 \\
\hline R15-3 & 48.39 & 0.07 & 32.32 & 0.00 & 0.61 & 0.01 & 0.14 & 15.25 & 2.59 \\
\hline R15-4 & 46.36 & 0.03 & 34.44 & 0.00 & 0.54 & 0.03 & 0.14 & 17.65 & 1.60 \\
\hline R15-11 & 48.03 & 0.04 & 33.88 & 0.01 & 0.49 & 0.02 & 0.14 & 17.07 & 1.84 \\
\hline R15-11 & 46.96 & 0.00 & 34.62 & 0.00 & 0.48 & 0.02 & 0.15 & 17.86 & 1.51 \\
\hline R15-11 & 47.37 & 0.05 & 34.13 & 0.01 & 0.47 & 0.01 & 0.16 & 17.41 & 1.62 \\
\hline R15-11 & 46.61 & 0.03 & 34.54 & 0.01 & 0.47 & 0.02 & 0.14 & 17.94 & 1.41 \\
\hline R15-11 & 46.62 & 0.01 & 34.72 & 0.00 & 0.50 & 0.00 & 0.13 & 18.25 & 1.35 \\
\hline R15-12 & 51.60 & 0.08 & 31.49 & 0.00 & 0.75 & 0.00 & 0.21 & 14.87 & 3.23 \\
\hline R15-12 & 51.45 & 0.02 & 30.88 & 0.00 & 0.78 & 0.00 & 0.24 & 13.89 & 3.46 \\
\hline R15-12 & 51.76 & 0.06 & 31.07 & 0.00 & 0.77 & 0.01 & 0.20 & 14.47 & 3.52 \\
\hline
\end{tabular}




$\begin{array}{llllllllll}\text { R15-14 } & 51.90 & 0.33 & 27.32 & 0.00 & 2.94 & 0.05 & 1.48 & 13.39 & 3.31 \\ \text { R15-14 } & 50.99 & 0.19 & 29.06 & 0.00 & 2.03 & 0.04 & 0.94 & 14.07 & 3.06 \\ \text { R15-13 } & 51.27 & 0.03 & 31.91 & 0.00 & 0.64 & 0.01 & 0.16 & 14.87 & 3.14 \\ \text { R15-13 } & 50.92 & 0.03 & 30.55 & 0.00 & 0.66 & 0.00 & 0.19 & 14.79 & 3.34 \\ \text { R15-13 } & 51.60 & 0.04 & 31.55 & 0.02 & 0.67 & 0.00 & 0.22 & 14.59 & 3.44 \\ \text { R15-15 } & 47.91 & 0.03 & 34.00 & 0.00 & 0.64 & 0.02 & 0.17 & 17.06 & 1.76 \\ \text { R15-15 } & 47.38 & 0.03 & 34.41 & 0.00 & 0.61 & 0.01 & 0.12 & 17.75 & 1.61 \\ \text { R15-15 } & 47.17 & 0.02 & 34.34 & 0.01 & 0.68 & 0.01 & 0.12 & 17.74 & 1.51 \\ \text { R15-15 } & 48.73 & 0.04 & 33.44 & 0.02 & 0.61 & 0.02 & 0.17 & 16.73 & 2.08 \\ \text { R15-15 } & 47.51 & 0.00 & 34.03 & 0.01 & 0.55 & 0.00 & 0.13 & 17.30 & 1.56 \\ \text { R15-15 } & 47.24 & 0.04 & 34.15 & 0.01 & 0.52 & 0.04 & 0.14 & 17.73 & 1.33 \\ \text { R15-15 } & 49.36 & 0.03 & 33.29 & 0.01 & 0.53 & 0.01 & 0.18 & 16.57 & 2.33 \\ \text { R15-16 } & 51.56 & 0.04 & 31.18 & 0.00 & 0.63 & 0.01 & 0.16 & 14.40 & 3.49 \\ \text { R15-16 } & 51.96 & 0.03 & 30.98 & 0.00 & 0.69 & 0.00 & 0.18 & 14.20 & 3.70\end{array}$

amphibole

\begin{tabular}{lrrrrrrrrr} 
& \multicolumn{1}{c}{$\mathrm{SiO} 2$} & \multicolumn{1}{c}{$\mathrm{TiO} 2$} & \multicolumn{1}{c}{$\mathrm{Al} 2 \mathrm{O} 3$} & $\mathrm{Cr} 2 \mathrm{O} 3$ & $\mathrm{FeO}$ & $\mathrm{MnO}$ & $\mathrm{MgO}$ & $\mathrm{CaO}$ & $\mathrm{Na} 2 \mathrm{O}$ \\
$\mathrm{R} 15-3$ & 41.58 & 1.04 & 13.48 & 0.01 & 16.49 & 0.29 & 10.21 & 11.47 & 2.03 \\
$\mathrm{R} 15-3$ & 41.65 & 1.06 & 13.24 & 0.00 & 16.27 & 0.30 & 10.05 & 11.33 & 2.07 \\
$\mathrm{R} 15-3$ & 41.41 & 1.02 & 13.41 & 0.01 & 16.53 & 0.32 & 10.26 & 11.40 & 1.88
\end{tabular}

ilmenite

\begin{tabular}{lrrrrrrrrr} 
& \multicolumn{1}{c}{$\mathrm{SiO} 2$} & \multicolumn{1}{c}{$\mathrm{TiO} 2$} & \multicolumn{1}{c}{$\mathrm{Al} 2 \mathrm{O} 3$} & \multicolumn{1}{c}{$\mathrm{Cr} 2 \mathrm{O} 3$} & \multicolumn{1}{l}{$\mathrm{FeO}$} & $\mathrm{MnO}$ & $\mathrm{MgO}$ & $\mathrm{CaO}$ & $\mathrm{Na} 2 \mathrm{O}$ \\
$\mathrm{R} 15-3$ & 0.02 & 45.77 & 1.92 & 0.01 & 49.59 & 1.88 & 0.13 & 0.12 & 0.04 \\
$\mathrm{R} 15-3$ & 6.23 & 45.58 & 1.75 & 0.02 & 36.98 & 1.78 & 1.21 & 4.82 & 0.05
\end{tabular}




\begin{tabular}{|c|c|c|c|c|c|c|c|}
\hline $\mathrm{K} 2 \mathrm{O}$ & P2O5 & Total & Wo & En & Fs & A & \\
\hline 0.00 & 0.02 & 99.71 & & 44.9 & 46.1 & 8.0 & 1.0 \\
\hline 0.00 & 0.02 & 100.06 & & 44.6 & 46.1 & 8.4 & 1.0 \\
\hline 0.00 & 0.02 & 99.96 & & 45.1 & 45.9 & 8.0 & 1.0 \\
\hline 0.00 & 0.00 & 100.18 & & 45.3 & 45.9 & 7.7 & 1.1 \\
\hline 0.00 & 0.03 & 100.11 & & 44.9 & 46.0 & 8.0 & 1.1 \\
\hline 0.00 & 0.00 & 99.60 & & 44.8 & 45.8 & 8.3 & 1.1 \\
\hline 0.00 & 0.00 & 99.85 & & 44.8 & 45.9 & 8.2 & 1.0 \\
\hline 0.01 & 0.01 & 99.86 & & 44.7 & 46.1 & 8.2 & 1.0 \\
\hline 0.00 & 0.00 & 99.72 & & 44.1 & 45.4 & 9.7 & 0.7 \\
\hline 0.00 & 0.03 & 99.78 & & 43.3 & 46.1 & 9.8 & 0.8 \\
\hline 0.01 & 0.01 & 99.77 & & 43.2 & 46.3 & 9.6 & 0.9 \\
\hline 0.00 & 0.05 & 99.51 & & 45.5 & 45.7 & 7.9 & 1.0 \\
\hline 0.01 & 0.03 & 100.14 & & 43.2 & 46.4 & 9.5 & 0.9 \\
\hline 0.00 & 0.00 & 99.92 & & 43.2 & 46.7 & 9.3 & 0.8 \\
\hline 0.01 & 0.00 & 99.20 & & 42.0 & 46.7 & 10.5 & 0.9 \\
\hline 0.00 & 0.00 & 99.55 & & 42.6 & 44.7 & 11.6 & 1.1 \\
\hline 0.01 & 0.00 & 100.35 & & 38.4 & 50.4 & 10.5 & 0.6 \\
\hline 0.01 & 0.00 & 99.81 & & 44.2 & 44.7 & 10.1 & 1.0 \\
\hline 0.01 & 0.00 & 100.27 & & 36.5 & 51.7 & 11.2 & 0.5 \\
\hline K2O & P2O5 & Total & Wo & En & Fs & A & \\
\hline 0.01 & 0.00 & 100.35 & & 41.7 & 47.4 & 10.1 & 0.8 \\
\hline 0.00 & 0.02 & 100.30 & & 37.6 & 51.3 & 10.5 & 0.7 \\
\hline 0.05 & 0.00 & 99.66 & & 43.1 & 44.3 & 9.7 & 3.0 \\
\hline 0.00 & 0.00 & 100.56 & & 44.2 & 44.8 & 10.0 & 1.0 \\
\hline 0.02 & 0.00 & 99.82 & & 43.9 & 45.1 & 10.0 & 0.9 \\
\hline 0.00 & 0.00 & 100.33 & & 43.9 & 45.0 & 10.2 & 0.9 \\
\hline 0.02 & 0.00 & 99.62 & & 43.6 & 45.5 & 9.8 & 1.1 \\
\hline 0.00 & 0.00 & 100.19 & & 44.5 & 44.7 & 9.8 & 1.0 \\
\hline K2O & P2O5 & Total & Wo & En & Fs & A & \\
\hline 0.00 & 0.00 & 100.28 & & 41.6 & 48.6 & 9.2 & 0.5 \\
\hline 0.00 & 0.00 & 99.24 & & 40.3 & 49.4 & 9.6 & 0.7 \\
\hline 0.00 & 0.00 & 99.58 & & 42.4 & 44.9 & 10.7 & 1.9 \\
\hline 0.00 & 0.00 & 100.99 & & 43.6 & 46.4 & 9.1 & 0.9 \\
\hline 0.00 & 0.00 & 101.02 & & 43.3 & 45.6 & 9.9 & 1.1 \\
\hline 0.00 & 0.00 & 99.12 & & 44.2 & 45.5 & 9.4 & 0.9 \\
\hline 0.01 & 0.04 & 99.06 & & 44.4 & 46.2 & 8.5 & 0.9 \\
\hline 0.00 & 0.00 & 100.34 & & 38.3 & 50.4 & 10.6 & 0.7 \\
\hline 0.01 & 0.00 & 100.97 & & 38.7 & 50.0 & 10.6 & 0.7 \\
\hline 0.00 & 0.00 & 100.54 & & 41.0 & 48.0 & 10.1 & 0.8 \\
\hline 0.00 & 0.00 & 100.40 & & 44.8 & 43.7 & 10.4 & 1.1 \\
\hline 0.01 & 0.00 & 101.01 & & 43.6 & 44.6 & 10.8 & 1.0 \\
\hline
\end{tabular}




\begin{tabular}{rrr}
\multicolumn{1}{l}{ K2O } & \multicolumn{1}{l}{ P2O5 } & \multicolumn{1}{l}{ Total } \\
0.02 & 0.05 & 99.84 \\
0.03 & 0.07 & 100.02 \\
0.03 & 0.02 & 99.72 \\
0.03 & 0.03 & 99.82 \\
0.03 & 0.01 & 99.43 \\
0.03 & 0.00 & 98.91 \\
0.02 & 0.06 & 99.32 \\
0.03 & 0.01 & 99.27 \\
0.02 & 0.01 & 99.27 \\
0.03 & 0.01 & 99.38 \\
0.02 & 0.00 & 98.97 \\
0.02 & 0.05 & 98.51 \\
0.01 & 0.03 & 99.11 \\
0.03 & 0.01 & 99.15 \\
0.02 & 0.13 & 98.72 \\
0.03 & 0.10 & 99.14 \\
0.03 & 0.08 & 99.54 \\
0.02 & 0.02 & 99.19 \\
0.03 & 0.00 & 99.25 \\
0.03 & 0.16 & 98.68 \\
0.03 & 0.02 & 99.84 \\
0.02 & 0.03 & 99.45 \\
0.01 & 0.02 & 99.81 \\
0.04 & 0.00 & 98.47 \\
0.05 & 0.02 & 100.04 \\
0.04 & 0.02 & 100.12 \\
0.02 & 0.01 & 100.08 \\
0.03 & 0.04 & 99.04 \\
& &
\end{tabular}

$\begin{array}{ccc}\text { An mol\% } & \text { Ab mol\% } & \text { Or mol\% } \\ 82.6 & 17.2 & 0.1 \\ 75.0 & 24.8 & 0.2 \\ 72.3 & 27.5 & 0.2 \\ 72.4 & 27.4 & 0.2 \\ 78.2 & 21.6 & 0.2 \\ 71.3 & 28.6 & 0.2 \\ 78.4 & 21.5 & 0.1 \\ 78.1 & 21.7 & 0.2 \\ 78.8 & 21.1 & 0.1 \\ 73.6 & 26.3 & 0.2 \\ 75.9 & 24.0 & 0.1 \\ 74.4 & 25.5 & 0.1 \\ 72.0 & 28.0 & 0.1 \\ 74.9 & 24.9 & 0.2 \\ 72.6 & 27.3 & 0.1 \\ 73.4 & 26.5 & 0.2 \\ 73.6 & 26.2 & 0.2 \\ 74.9 & 25.0 & 0.1 \\ 74.8 & 25.1 & 0.2 \\ 75.6 & 24.2 & 0.2 \\ 74.0 & 25.8 & 0.2 \\ 82.4 & 17.5 & 0.1 \\ 74.4 & 25.6 & 0.1 \\ 70.0 & 29.7 & 0.2 \\ 68.0 & 31.7 & 0.3 \\ 66.9 & 32.8 & 0.2 \\ 77.0 & 22.9 & 0.1 \\ 74.6 & 25.2 & 0.2 \\ & & \end{array}$

\begin{tabular}{lrr}
\multicolumn{1}{l}{ K2O } & \multicolumn{1}{l}{ P2O5 } & \multicolumn{1}{l}{ Total } \\
0.03 & 0.03 & 98.62 \\
0.03 & 0.00 & 99.31 \\
0.04 & 0.00 & 99.12 \\
0.03 & 0.01 & 98.93 \\
0.04 & 0.00 & 98.88 \\
0.03 & 0.02 & 99.36 \\
0.03 & 0.00 & 98.19 \\
0.03 & 0.00 & 98.70 \\
0.02 & 0.01 & 98.30 \\
0.05 & 0.00 & 99.41 \\
0.04 & 0.02 & 98.56 \\
0.05 & 0.02 & 98.62 \\
0.02 & 0.00 & 98.54 \\
0.00 & 0.01 & 99.38 \\
0.00 & 0.04 & 98.27
\end{tabular}

An mol\% Ab mol\% Or mol\%

$\begin{array}{rrr}69.4 & 30.4 & 0.2 \\ 71.2 & 28.6 & 0.2 \\ 70.9 & 28.9 & 0.2 \\ 72.3 & 27.5 & 0.2 \\ 71.3 & 28.5 & 0.3 \\ 69.6 & 30.2 & 0.2 \\ 68.2 & 31.6 & 0.2 \\ 72.8 & 27.0 & 0.2 \\ 73.6 & 26.2 & 0.1 \\ 69.4 & 30.3 & 0.3 \\ 69.0 & 30.7 & 0.3 \\ 70.7 & 29.1 & 0.3 \\ 86.9 & 13.0 & 0.1 \\ 93.5 & 6.5 & 0.0 \\ 94.7 & 5.3 & 0.0\end{array}$




\begin{tabular}{|c|c|c|c|c|c|}
\hline 0.01 & 0.03 & 99.23 & 94.7 & 5.3 & 0.1 \\
\hline 0.00 & 0.01 & 99.31 & 93.6 & 6.4 & 0.0 \\
\hline 0.02 & 0.05 & 99.29 & 82.8 & 17.1 & 0.1 \\
\hline 0.02 & 0.02 & 99.52 & 86.2 & 13.7 & 0.1 \\
\hline 0.01 & 0.00 & 99.09 & 76.2 & 23.7 & 0.0 \\
\hline 0.03 & 0.01 & 99.20 & 75.5 & 24.3 & 0.2 \\
\hline 0.03 & 0.00 & 101.32 & 71.6 & 28.2 & 0.2 \\
\hline 0.03 & 0.00 & 101.05 & 71.5 & 28.3 & 0.2 \\
\hline 0.00 & 0.00 & 100.71 & 88.2 & 11.8 & 0.0 \\
\hline 0.01 & 0.00 & 101.39 & 87.8 & 12.2 & 0.0 \\
\hline 0.03 & 0.00 & 101.19 & 74.1 & 25.8 & 0.1 \\
\hline 0.02 & 0.00 & 100.96 & 74.8 & 25.0 & 0.1 \\
\hline $\mathrm{K} 2 \mathrm{O}$ & P2O5 & Total & An mol\% & $\mathrm{Ab} \mathrm{mol} \%$ & Or mol\% \\
\hline 0.02 & 0.01 & 99.68 & 79.2 & 20.7 & 0.1 \\
\hline 0.02 & 0.03 & 100.17 & 77.2 & 22.7 & 0.1 \\
\hline 0.01 & 0.01 & 99.51 & 78.8 & 21.1 & 0.1 \\
\hline 0.03 & 0.03 & 99.01 & 75.7 & 24.1 & 0.2 \\
\hline 0.01 & 0.01 & 99.27 & 79.3 & 20.6 & 0.1 \\
\hline 0.04 & 0.03 & 99.44 & 72.4 & 27.3 & 0.2 \\
\hline 0.03 & 0.11 & 99.50 & 75.4 & 24.4 & 0.2 \\
\hline 0.03 & 0.00 & 99.14 & 74.8 & 25.1 & 0.2 \\
\hline 0.02 & 0.02 & 99.30 & 77.0 & 22.8 & 0.1 \\
\hline 0.02 & 0.01 & 99.24 & 76.8 & 23.1 & 0.1 \\
\hline 0.03 & 0.02 & 98.48 & 77.0 & 22.8 & 0.2 \\
\hline 0.01 & 0.03 & 99.06 & 86.5 & 13.5 & 0.0 \\
\hline 0.03 & 0.02 & 98.45 & 75.3 & 24.5 & 0.2 \\
\hline 0.03 & 0.01 & 99.23 & 71.5 & 28.3 & 0.2 \\
\hline 0.04 & 0.02 & 98.58 & 67.6 & 32.1 & 0.2 \\
\hline 0.02 & 0.04 & 98.92 & 73.9 & 25.9 & 0.1 \\
\hline 0.04 & 0.05 & 98.70 & 70.7 & 29.0 & 0.3 \\
\hline 0.03 & 0.01 & 99.40 & 71.2 & 28.6 & 0.2 \\
\hline 0.04 & 0.00 & 99.13 & 68.5 & 31.3 & 0.2 \\
\hline 0.04 & 0.06 & 99.37 & 68.9 & 30.9 & 0.3 \\
\hline 0.01 & 0.03 & 99.31 & 84.9 & 15.0 & 0.1 \\
\hline 0.00 & 0.01 & 99.41 & 87.5 & 12.5 & 0.0 \\
\hline 0.09 & 0.05 & 98.27 & 73.3 & 26.0 & 0.6 \\
\hline 0.03 & 0.03 & 99.45 & 76.4 & 23.5 & 0.2 \\
\hline 0.02 & 0.04 & 100.88 & 85.8 & 14.1 & 0.1 \\
\hline 0.02 & 0.00 & 101.64 & 83.6 & 16.3 & 0.1 \\
\hline 0.00 & 0.00 & 101.68 & 86.7 & 13.3 & 0.0 \\
\hline 0.02 & 0.00 & 101.34 & 85.5 & 14.4 & 0.1 \\
\hline 0.01 & 0.00 & 101.20 & 87.5 & 12.4 & 0.1 \\
\hline 0.01 & 0.00 & 101.61 & 88.1 & 11.8 & 0.1 \\
\hline 0.04 & 0.00 & 102.30 & 71.6 & 28.1 & 0.2 \\
\hline 0.03 & 0.00 & 100.76 & 68.8 & 31.0 & 0.2 \\
\hline 0.02 & 0.00 & 101.93 & 69.4 & 30.5 & 0.1 \\
\hline
\end{tabular}




$\begin{array}{lll}0.11 & 0.00 & 100.89 \\ 0.07 & 0.00 & 100.51 \\ 0.03 & 0.00 & 102.08 \\ 0.05 & 0.00 & 100.56 \\ 0.05 & 0.00 & 102.26 \\ 0.03 & 0.00 & 101.62 \\ 0.02 & 0.00 & 101.99 \\ 0.02 & 0.00 & 101.65 \\ 0.02 & 0.00 & 101.85 \\ 0.02 & 0.00 & 101.14 \\ 0.02 & 0.00 & 101.24 \\ 0.03 & 0.00 & 102.36 \\ 0.02 & 0.00 & 101.49 \\ 0.04 & 0.00 & 101.85\end{array}$

$\begin{array}{lll}68.7 & 30.7 & 0.6 \\ 71.4 & 28.1 & 0.4 \\ 72.3 & 27.6 & 0.2 \\ 70.8 & 29.0 & 0.3 \\ 69.9 & 29.8 & 0.3 \\ 84.1 & 15.7 & 0.2 \\ 85.8 & 14.1 & 0.1 \\ 86.6 & 13.3 & 0.1 \\ 81.5 & 18.4 & 0.1 \\ 85.9 & 14.0 & 0.1 \\ 87.9 & 11.9 & 0.1 \\ 79.6 & 20.2 & 0.1 \\ 69.4 & 30.4 & 0.1 \\ 67.8 & 32.0 & 0.2\end{array}$

\begin{tabular}{|c|c|c|c|c|c|}
\hline $\mathrm{K} 2 \mathrm{O}$ & P2O5 & $\mathrm{F}$ & $\mathrm{Cl}$ & $\mathrm{SO} 3$ & Total \\
\hline 0.32 & 0.03 & 0.08 & 0.13 & 0.00 & 97.10 \\
\hline 0.33 & 0.01 & 0.04 & 0.11 & 0.00 & 96.42 \\
\hline 0.33 & 0.04 & 0.01 & 0.12 & 0.02 & 96.73 \\
\hline
\end{tabular}

\begin{tabular}{rrr}
\multicolumn{1}{r}{ K2O } & \multicolumn{1}{c}{$\mathrm{P} 2 \mathrm{O} 5$} & \multicolumn{1}{l}{ Total } \\
0.00 & 0.05 & 99.52 \\
0.04 & 0.07 & 98.59
\end{tabular}


Supplementary 2. Electron microprobe analyses for minerals in glass

\begin{tabular}{|c|c|c|c|c|c|c|c|c|c|}
\hline R05-cpx & $\mathrm{SiO} 2$ & TiO2 & $\mathrm{Al} 2 \mathrm{O} 3$ & $\mathrm{Cr} 2 \mathrm{O} 3$ & $\mathrm{FeO}$ & $\mathrm{MnO}$ & $\mathrm{MgO}$ & $\mathrm{CaO}$ & $\mathrm{Na} 2 \mathrm{O}$ \\
\hline R05-18 & 52.59 & 0.56 & 2.74 & 0.31 & 4.88 & 0.13 & 16.22 & 21.98 & 0.28 \\
\hline R05-18 & 52.49 & 0.54 & 2.77 & 0.31 & 5.16 & 0.14 & 16.33 & 21.98 & 0.27 \\
\hline R05-18 & 52.58 & 0.55 & 2.59 & 0.32 & 4.94 & 0.10 & 16.28 & 22.28 & 0.29 \\
\hline R05-18 & 52.61 & 0.58 & 2.60 & 0.31 & 4.80 & 0.12 & 16.34 & 22.46 & 0.30 \\
\hline R05-18 & 52.60 & 0.59 & 2.72 & 0.34 & 4.93 & 0.13 & 16.32 & 22.15 & 0.30 \\
\hline R05-18 & 52.75 & 0.55 & 2.54 & 0.29 & 5.06 & 0.12 & 16.09 & 21.90 & 0.30 \\
\hline R05-18 & 52.63 & 0.53 & 2.53 & 0.30 & 5.10 & 0.10 & 16.27 & 22.11 & 0.28 \\
\hline R05-18 & 52.67 & 0.51 & 2.61 & 0.29 & 5.06 & 0.11 & 16.31 & 22.03 & 0.26 \\
\hline R05-18 & 51.36 & 0.64 & 3.78 & 0.23 & 5.94 & 0.15 & 15.91 & 21.48 & 0.20 \\
\hline R05-18 & 51.70 & 0.60 & 3.73 & 0.20 & 5.93 & 0.16 & 16.12 & 21.07 & 0.22 \\
\hline R05-18 & 51.83 & 0.56 & 3.33 & 0.24 & 5.86 & 0.17 & 16.32 & 21.17 & 0.23 \\
\hline R05-18 & 52.06 & 0.58 & 2.74 & 0.33 & 4.88 & 0.08 & 16.14 & 22.38 & 0.27 \\
\hline R05-18 & 52.15 & 0.57 & 3.27 & 0.28 & 5.84 & 0.13 & 16.39 & 21.20 & 0.25 \\
\hline R05-18 & 52.19 & 0.49 & 3.17 & 0.33 & 5.72 & 0.14 & 16.45 & 21.19 & 0.23 \\
\hline R05-cpx1 & 50.97 & 0.65 & 4.09 & 0.24 & 6.39 & 0.14 & 16.19 & 20.28 & 0.23 \\
\hline $\mathrm{R} 05-c p \times 2$ & 50.23 & 1.03 & 5.46 & 0.10 & 6.87 & 0.17 & 15.19 & 20.14 & 0.28 \\
\hline R05-cpx2 & 53.46 & 0.38 & 2.19 & 0.14 & 6.48 & 0.20 & 18.07 & 19.18 & 0.18 \\
\hline R05-cpx2 & 51.14 & 0.74 & 4.47 & 0.28 & 6.10 & 0.11 & 15.44 & 21.22 & 0.27 \\
\hline R05-cpx2 & 53.37 & 0.41 & 2.27 & 0.15 & 6.95 & 0.21 & 18.51 & 18.18 & 0.15 \\
\hline
\end{tabular}

\begin{tabular}{lrrrrrrrrr} 
R06-cpx & \multicolumn{1}{l}{$\mathrm{SiO2}$} & $\mathrm{TiO} 2$ & $\mathrm{Al} 2 \mathrm{O} 3$ & $\mathrm{Cr} 2 \mathrm{O} 3$ & \multicolumn{1}{l}{$\mathrm{FeO}$} & $\mathrm{MnO}$ & $\mathrm{MgO}$ & $\mathrm{CaO}$ & $\mathrm{Na} 2 \mathrm{O}$ \\
R06-14 & 51.65 & 0.60 & 4.08 & 0.48 & 6.19 & 0.13 & 16.61 & 20.36 & 0.23 \\
R06-14 & 53.43 & 0.33 & 2.19 & 0.15 & 6.53 & 0.18 & 18.44 & 18.80 & 0.20 \\
R06-13 & 51.36 & 0.66 & 6.92 & 0.45 & 5.44 & 0.12 & 14.35 & 19.43 & 0.74 \\
R6-14-2 & 51.46 & 0.74 & 4.15 & 0.32 & 6.17 & 0.14 & 15.72 & 21.60 & 0.26 \\
R6-14-2 & 51.66 & 0.62 & 3.92 & 0.35 & 6.06 & 0.14 & 15.63 & 21.18 & 0.24 \\
R6-14-2 & 51.10 & 0.84 & 4.63 & 0.33 & 6.20 & 0.15 & 15.63 & 21.20 & 0.24 \\
R6-14-2 & 50.51 & 0.60 & 4.73 & 0.38 & 5.98 & 0.12 & 15.84 & 21.12 & 0.29 \\
R6-14-2 & 50.84 & 0.79 & 4.67 & 0.42 & 6.00 & 0.12 & 15.55 & 21.53 & 0.28 \\
& & & & & & & & & \\
R15-cpx & SiO2 & TiO2 & Al2O3 & Cr2O3 & FeO & MnO & MgO & CaO & Na20 \\
R15-12 & 53.56 & 0.35 & 1.78 & 0.26 & 5.74 & 0.16 & 17.46 & 20.79 & 0.15 \\
R15-12 & 52.66 & 0.39 & 2.36 & 0.36 & 5.87 & 0.17 & 17.43 & 19.78 & 0.18 \\
R15-12 & 51.05 & 0.73 & 5.41 & 0.20 & 6.32 & 0.13 & 15.20 & 19.98 & 0.50 \\
R15-16 & 51.81 & 0.58 & 4.02 & 0.45 & 5.72 & 0.08 & 16.49 & 21.59 & 0.25 \\
R15-16 & 51.48 & 0.54 & 4.40 & 0.12 & 6.17 & 0.16 & 16.29 & 21.53 & 0.30 \\
R15-3 & 50.43 & 0.53 & 4.42 & 0.34 & 5.75 & 0.13 & 15.84 & 21.42 & 0.24 \\
R15-3 & 50.94 & 0.56 & 3.51 & 0.54 & 5.19 & 0.12 & 16.22 & 21.68 & 0.26 \\
R15-13 & 53.27 & 0.33 & 2.18 & 0.08 & 6.65 & 0.20 & 18.17 & 19.22 & 0.20 \\
R15-13 & 53.51 & 0.36 & 2.44 & 0.10 & 6.64 & 0.17 & 18.05 & 19.45 & 0.20 \\
R15-13 & 53.04 & 0.35 & 2.73 & 0.09 & 6.28 & 0.19 & 17.18 & 20.42 & 0.21 \\
R15-13 & 51.46 & 0.69 & 4.49 & 0.18 & 6.28 & 0.17 & 15.16 & 21.64 & 0.29 \\
R15-13 & 51.34 & 0.79 & 4.70 & 0.14 & 6.60 & 0.18 & 15.63 & 21.29 & 0.27
\end{tabular}




\begin{tabular}{|c|c|c|}
\hline K2O & P2O5 & Total \\
\hline 0.00 & 0.02 & 99.71 \\
\hline 0.00 & 0.02 & 100.06 \\
\hline 0.00 & 0.02 & 99.96 \\
\hline 0.00 & 0.00 & 100.18 \\
\hline 0.00 & 0.03 & 100.11 \\
\hline 0.00 & 0.00 & 99.60 \\
\hline 0.00 & 0.00 & 99.85 \\
\hline 0.01 & 0.01 & 99.86 \\
\hline 0.00 & 0.00 & 99.72 \\
\hline 0.00 & 0.03 & 99.78 \\
\hline 0.01 & 0.01 & 99.77 \\
\hline 0.00 & 0.05 & 99.51 \\
\hline 0.01 & 0.03 & 100.14 \\
\hline 0.00 & 0.00 & 99.92 \\
\hline 0.01 & 0.00 & 99.20 \\
\hline 0.00 & 0.00 & 99.55 \\
\hline 0.01 & 0.00 & 100.35 \\
\hline 0.01 & 0.00 & 99.81 \\
\hline 0.01 & 0.00 & 100.27 \\
\hline
\end{tabular}

\begin{tabular}{cccc} 
Wo En & \multicolumn{2}{c}{ Fs } & \multicolumn{2}{c}{ Ac } \\
44.9 & 46.1 & 8.0 & 1.0 \\
44.6 & 46.1 & 8.4 & 1.0 \\
45.1 & 45.9 & 8.0 & 1.0 \\
45.3 & 45.9 & 7.7 & 1.1 \\
44.9 & 46.0 & 8.0 & 1.1 \\
44.8 & 45.8 & 8.3 & 1.1 \\
44.8 & 45.9 & 8.2 & 1.0 \\
44.7 & 46.1 & 8.2 & 1.0 \\
44.1 & 45.4 & 9.7 & 0.7 \\
43.3 & 46.1 & 9.8 & 0.8 \\
43.2 & 46.3 & 9.6 & 0.9 \\
45.5 & 45.7 & 7.9 & 1.0 \\
43.2 & 46.4 & 9.5 & 0.9 \\
43.2 & 46.7 & 9.3 & 0.8 \\
42.0 & 46.7 & 10.5 & 0.9 \\
42.6 & 44.7 & 11.6 & 1.1 \\
38.4 & 50.4 & 10.5 & 0.6 \\
44.2 & 44.7 & 10.1 & 1.0 \\
36.5 & 51.7 & 11.2 & 0.5
\end{tabular}

\begin{tabular}{rrr}
\multicolumn{1}{l}{ K2O } & \multicolumn{1}{l}{ P2O5 } & \multicolumn{1}{l}{ Total } \\
0.01 & 0.00 & 100.35 \\
0.00 & 0.02 & 100.30 \\
0.05 & 0.00 & 99.66 \\
0.00 & 0.00 & 100.56 \\
0.02 & 0.00 & 99.82 \\
0.00 & 0.00 & 100.33 \\
0.02 & 0.00 & 99.62 \\
0.00 & 0.00 & 100.19
\end{tabular}

\begin{tabular}{crrr} 
Wo & En & Fs & \multicolumn{2}{c}{ Ac } \\
41.7 & 47.4 & 10.1 & 0.8 \\
37.6 & 51.3 & 10.5 & 0.7 \\
43.1 & 44.3 & 9.7 & 3.0 \\
44.2 & 44.8 & 10.0 & 1.0 \\
43.9 & 45.1 & 10.0 & 0.9 \\
43.9 & 45.0 & 10.2 & 0.9 \\
43.6 & 45.5 & 9.8 & 1.1 \\
44.5 & 44.7 & 9.8 & 1.0
\end{tabular}

\begin{tabular}{rrr}
\multicolumn{1}{l}{ K2O } & \multicolumn{1}{l}{ P2O5 } & \multicolumn{1}{l}{ Total } \\
0.00 & 0.00 & 100.28 \\
0.00 & 0.00 & 99.24 \\
0.00 & 0.00 & 99.58 \\
0.00 & 0.00 & 100.99 \\
0.00 & 0.00 & 101.02 \\
0.00 & 0.00 & 99.12 \\
0.01 & 0.04 & 99.06 \\
0.00 & 0.00 & 100.34 \\
0.01 & 0.00 & 100.97 \\
0.00 & 0.00 & 100.54 \\
0.00 & 0.00 & 100.40 \\
0.01 & 0.00 & 101.01
\end{tabular}

\begin{tabular}{crrr} 
Wo En & \multicolumn{2}{c}{ Fs } \\
41.6 & 48.6 & 9.2 & 0.5 \\
40.3 & 49.4 & 9.6 & 0.7 \\
42.4 & 44.9 & 10.7 & 1.9 \\
43.6 & 46.4 & 9.1 & 0.9 \\
43.3 & 45.6 & 9.9 & 1.1 \\
44.2 & 45.5 & 9.4 & 0.9 \\
44.4 & 46.2 & 8.5 & 0.9 \\
38.3 & 50.4 & 10.6 & 0.7 \\
38.7 & 50.0 & 10.6 & 0.7 \\
41.0 & 48.0 & 10.1 & 0.8 \\
44.8 & 43.7 & 10.4 & 1.1 \\
43.6 & 44.6 & 10.8 & 1.0
\end{tabular}




\begin{tabular}{|c|c|c|c|c|c|c|c|c|c|}
\hline R05-pl & $\mathrm{SiO} 2$ & TiO2 & $\mathrm{Al} 2 \mathrm{O} 3$ & $\mathrm{Cr} 2 \mathrm{O} 3$ & $\mathrm{FeO}$ & $\mathrm{MnO}$ & $\mathrm{MgO}$ & $\mathrm{CaO}$ & $\mathrm{Na} 2 \mathrm{O}$ \\
\hline R05-1 & 47.27 & 0.04 & 33.56 & 0.00 & 0.61 & 0.00 & 0.15 & 16.23 & 1.87 \\
\hline R05-1 & 48.97 & 0.04 & 32.29 & 0.01 & 0.67 & 0.01 & 0.17 & 15.00 & 2.74 \\
\hline R05-1 & 49.61 & 0.03 & 31.65 & 0.00 & 0.66 & 0.02 & 0.17 & 14.41 & 3.04 \\
\hline R05-1 & 49.42 & 0.05 & 31.91 & 0.02 & 0.70 & 0.05 & 0.16 & 14.41 & 3.02 \\
\hline R05-2 & 47.91 & 0.00 & 32.73 & 0.02 & 0.50 & 0.01 & 0.20 & 15.64 & 2.38 \\
\hline R05-2 & 50.03 & 0.06 & 31.16 & 0.00 & 0.50 & 0.01 & 0.20 & 13.83 & 3.07 \\
\hline R05-2 & 47.95 & 0.00 & 32.64 & 0.00 & 0.51 & 0.02 & 0.16 & 15.60 & 2.36 \\
\hline R05-2 & 47.98 & 0.03 & 32.68 & 0.00 & 0.52 & 0.01 & 0.17 & 15.47 & 2.37 \\
\hline R05-2 & 47.99 & 0.03 & 32.68 & 0.00 & 0.56 & 0.00 & 0.18 & 15.49 & 2.29 \\
\hline R05-2 & 48.94 & 0.04 & 31.85 & 0.01 & 0.59 & 0.04 & 0.20 & 14.73 & 2.90 \\
\hline R05-2 & 48.98 & 0.05 & 31.64 & 0.00 & 0.62 & 0.00 & 0.21 & 14.80 & 2.58 \\
\hline R05-3 & 49.11 & 0.07 & 31.52 & 0.01 & 0.64 & 0.00 & 0.17 & 14.20 & 2.69 \\
\hline R05-3 & 49.79 & 0.07 & 31.16 & 0.04 & 0.72 & 0.01 & 0.19 & 14.07 & 3.02 \\
\hline R05-3 & 48.97 & 0.01 & 31.65 & 0.01 & 0.72 & 0.02 & 0.14 & 14.81 & 2.72 \\
\hline R05-3 & 49.59 & 0.04 & 30.98 & 0.00 & 0.64 & 0.00 & 0.19 & 14.14 & 2.93 \\
\hline R05-3 & 49.23 & 0.02 & 31.65 & 0.01 & 0.67 & 0.00 & 0.13 & 14.43 & 2.88 \\
\hline R05-3 & 49.27 & 0.01 & 31.84 & 0.00 & 0.64 & 0.01 & 0.13 & 14.64 & 2.88 \\
\hline R05-3 & 49.02 & 0.06 & 31.81 & 0.00 & 0.71 & 0.00 & 0.15 & 14.69 & 2.71 \\
\hline R05-3 & 48.81 & 0.02 & 32.13 & 0.00 & 0.63 & 0.00 & 0.15 & 14.75 & 2.73 \\
\hline R05-3 & 48.34 & 0.00 & 32.18 & 0.00 & 0.75 & 0.01 & 0.16 & 14.48 & 2.56 \\
\hline R05-3 & 49.36 & 0.04 & 31.84 & 0.00 & 0.61 & 0.02 & 0.17 & 14.85 & 2.86 \\
\hline R05-4 & 46.89 & 0.04 & 33.38 & 0.00 & 0.60 & 0.00 & 0.20 & 16.36 & 1.92 \\
\hline R05-4 & 48.89 & 0.04 & 32.23 & 0.00 & 0.64 & 0.01 & 0.16 & 14.96 & 2.84 \\
\hline R05-4 & 50.01 & 0.04 & 30.88 & 0.00 & 0.67 & 0.01 & 0.17 & 13.48 & 3.16 \\
\hline R05-4 & 51.28 & 0.05 & 30.90 & 0.03 & 0.79 & 0.00 & 0.16 & 13.35 & 3.43 \\
\hline R05-4 & 51.26 & 0.05 & 30.84 & 0.00 & 0.80 & 0.03 & 0.16 & 13.31 & 3.61 \\
\hline R05-4 & 48.77 & 0.01 & 32.55 & 0.00 & 0.64 & 0.02 & 0.15 & 15.32 & 2.52 \\
\hline R05-4 & 49.08 & 0.02 & 31.48 & 0.00 & 0.67 & 0.00 & 0.21 & 14.72 & 2.75 \\
\hline R06-pl & $\mathrm{SiO} 2$ & TiO2 & Al2O3 & $\mathrm{Cr} 2 \mathrm{O} 3$ & $\mathrm{FeO}$ & $\mathrm{MnO}$ & $\mathrm{MgO}$ & $\mathrm{CaO}$ & $\mathrm{Na} 2 \mathrm{O}$ \\
\hline R06-1 & 49.88 & 0.06 & 30.72 & 0.02 & 0.70 & 0.00 & 0.18 & 13.69 & 3.31 \\
\hline R06-1 & 49.80 & 0.05 & 31.31 & 0.00 & 0.61 & 0.00 & 0.18 & 14.13 & 3.13 \\
\hline R06-2 & 49.74 & 0.06 & 31.19 & 0.00 & 0.63 & 0.02 & 0.18 & 14.08 & 3.17 \\
\hline R06-2 & 49.32 & 0.04 & 31.21 & 0.00 & 0.67 & 0.01 & 0.16 & 14.42 & 3.03 \\
\hline R06-2 & 49.99 & 0.07 & 30.90 & 0.00 & 0.67 & 0.00 & 0.18 & 13.92 & 3.08 \\
\hline R06-2 & 50.10 & 0.04 & 30.98 & 0.00 & 0.75 & 0.03 & 0.16 & 13.87 & 3.33 \\
\hline R06-2 & 49.90 & 0.03 & 30.61 & 0.01 & 0.67 & 0.02 & 0.20 & 13.27 & 3.40 \\
\hline R06-2 & 48.83 & 0.03 & 31.56 & 0.00 & 0.68 & 0.03 & 0.16 & 14.42 & 2.96 \\
\hline R06-2 & 48.76 & 0.02 & 31.55 & 0.00 & 0.70 & 0.01 & 0.16 & 14.20 & 2.80 \\
\hline R06-2 & 50.27 & 0.05 & 31.18 & 0.00 & 0.53 & 0.02 & 0.16 & 13.74 & 3.32 \\
\hline R06-2 & 49.79 & 0.04 & 30.95 & 0.00 & 0.53 & 0.01 & 0.14 & 13.66 & 3.36 \\
\hline R06-2 & 49.86 & 0.05 & 30.73 & 0.02 & 0.56 & 0.03 & 0.14 & 13.97 & 3.17 \\
\hline R06-3 & 45.88 & 0.01 & 33.48 & 0.01 & 0.41 & 0.00 & 0.18 & 17.12 & 1.42 \\
\hline R06-3 & 43.90 & 0.00 & 35.64 & 0.01 & 0.35 & 0.00 & 0.13 & 18.62 & 0.72 \\
\hline R06-3 & 43.50 & 0.00 & 35.69 & 0.00 & 0.34 & 0.03 & 0.09 & 18.01 & 0.56 \\
\hline
\end{tabular}




\begin{tabular}{|c|c|c|c|c|c|c|c|c|c|}
\hline R06-3 & 43.96 & 0.01 & 35.62 & 0.01 & 0.33 & 0.00 & 0.09 & 18.60 & 0.57 \\
\hline R06-3 & 43.73 & 0.01 & 35.74 & 0.00 & 0.35 & 0.00 & 0.09 & 18.67 & 0.70 \\
\hline R06-3 & 46.66 & 0.02 & 33.66 & 0.01 & 0.50 & 0.00 & 0.18 & 16.32 & 1.86 \\
\hline R06-3 & 46.22 & 0.02 & 33.88 & 0.02 & 0.49 & 0.00 & 0.17 & 17.16 & 1.51 \\
\hline R06-3 & 48.69 & 0.01 & 32.15 & 0.00 & 0.54 & 0.00 & 0.20 & 14.93 & 2.57 \\
\hline R06-3 & 48.94 & 0.07 & 32.05 & 0.00 & 0.53 & 0.00 & 0.19 & 14.76 & 2.62 \\
\hline R06-12 & 50.96 & 0.04 & 31.41 & 0.00 & 0.59 & 0.03 & 0.22 & 14.77 & 3.21 \\
\hline R06-12 & 50.85 & 0.02 & 31.63 & 0.00 & 0.63 & 0.00 & 0.20 & 14.48 & 3.17 \\
\hline R06-12 & 46.40 & 0.01 & 34.55 & 0.00 & 0.45 & 0.00 & 0.16 & 17.82 & 1.32 \\
\hline R06-12 & 46.69 & 0.01 & 34.62 & 0.00 & 0.48 & 0.01 & 0.16 & 17.99 & 1.38 \\
\hline R06-12 & 50.31 & 0.03 & 31.65 & 0.01 & 0.60 & 0.02 & 0.24 & 15.34 & 2.95 \\
\hline R06-12 & 50.18 & 0.00 & 31.64 & 0.00 & 0.63 & 0.00 & 0.22 & 15.41 & 2.85 \\
\hline R15-pl & $\mathrm{SiO} 2$ & TiO2 & $\mathrm{Al} 2 \mathrm{O} 3$ & $\mathrm{Cr} 2 \mathrm{O} 3$ & $\mathrm{FeO}$ & $\mathrm{MnO}$ & MgO & $\mathrm{CaO}$ & $\mathrm{Na} 2 \mathrm{O}$ \\
\hline R15-1 & 47.79 & 0.00 & 32.87 & 0.01 & 0.60 & 0.04 & 0.20 & 15.82 & 2.29 \\
\hline R15-1 & 48.66 & 0.05 & 32.65 & 0.00 & 0.59 & 0.00 & 0.21 & 15.43 & 2.51 \\
\hline R15-1 & 47.93 & 0.03 & 32.74 & 0.00 & 0.52 & 0.01 & 0.18 & 15.73 & 2.33 \\
\hline R15-1 & 48.72 & 0.05 & 31.88 & 0.01 & 0.55 & 0.01 & 0.21 & 14.89 & 2.62 \\
\hline R15-1 & 47.76 & 0.03 & 32.90 & 0.01 & 0.53 & 0.00 & 0.16 & 15.60 & 2.24 \\
\hline R15-1 & 49.53 & 0.05 & 31.67 & 0.00 & 0.66 & 0.01 & 0.15 & 14.31 & 2.98 \\
\hline R15-1 & 48.87 & 0.05 & 31.99 & 0.03 & 0.67 & 0.03 & 0.16 & 14.85 & 2.66 \\
\hline R15-1 & 48.96 & 0.06 & 31.97 & 0.00 & 0.53 & 0.01 & 0.15 & 14.70 & 2.72 \\
\hline R15-1 & 48.28 & 0.03 & 32.39 & 0.01 & 0.56 & 0.01 & 0.17 & 15.29 & 2.50 \\
\hline R15-2 & 48.47 & 0.03 & 32.13 & 0.01 & 0.62 & 0.00 & 0.16 & 15.24 & 2.53 \\
\hline R15-2 & 47.92 & 0.05 & 32.46 & 0.00 & 0.52 & 0.01 & 0.17 & 14.86 & 2.43 \\
\hline R15-2 & 46.00 & 0.00 & 33.87 & 0.01 & 0.48 & 0.00 & 0.13 & 17.07 & 1.47 \\
\hline R15-2 & 49.69 & 0.03 & 31.08 & 0.01 & 0.68 & 0.00 & 0.17 & 14.15 & 2.54 \\
\hline R15-2 & 49.76 & 0.06 & 31.48 & 0.02 & 0.67 & 0.03 & 0.17 & 13.93 & 3.05 \\
\hline R15-2 & 50.32 & 0.08 & 30.24 & 0.00 & 0.64 & 0.02 & 0.15 & 13.46 & 3.53 \\
\hline R15-2 & 49.15 & 0.04 & 31.64 & 0.00 & 0.62 & 0.02 & 0.18 & 14.38 & 2.79 \\
\hline R15-3 & 49.83 & 0.02 & 30.83 & 0.01 & 0.60 & 0.03 & 0.16 & 13.96 & 3.16 \\
\hline R15-3 & 49.86 & 0.05 & 31.42 & 0.00 & 0.61 & 0.00 & 0.18 & 14.08 & 3.12 \\
\hline R15-3 & 50.30 & 0.03 & 31.11 & 0.00 & 0.63 & 0.01 & 0.15 & 13.43 & 3.39 \\
\hline R15-3 & 50.18 & 0.07 & 30.93 & 0.01 & 0.77 & 0.01 & 0.17 & 13.70 & 3.39 \\
\hline R15-3 & 45.86 & 0.04 & 34.23 & 0.00 & 0.57 & 0.00 & 0.13 & 16.79 & 1.64 \\
\hline R15-3 & 45.77 & 0.03 & 34.17 & 0.01 & 0.58 & 0.00 & 0.11 & 17.32 & 1.36 \\
\hline R15-3 & 49.58 & 0.33 & 28.45 & 0.00 & 2.43 & 0.06 & 1.36 & 13.24 & 2.60 \\
\hline R15-3 & 48.39 & 0.07 & 32.32 & 0.00 & 0.61 & 0.01 & 0.14 & 15.25 & 2.59 \\
\hline R15-4 & 46.36 & 0.03 & 34.44 & 0.00 & 0.54 & 0.03 & 0.14 & 17.65 & 1.60 \\
\hline R15-11 & 48.03 & 0.04 & 33.88 & 0.01 & 0.49 & 0.02 & 0.14 & 17.07 & 1.84 \\
\hline R15-11 & 46.96 & 0.00 & 34.62 & 0.00 & 0.48 & 0.02 & 0.15 & 17.86 & 1.51 \\
\hline R15-11 & 47.37 & 0.05 & 34.13 & 0.01 & 0.47 & 0.01 & 0.16 & 17.41 & 1.62 \\
\hline R15-11 & 46.61 & 0.03 & 34.54 & 0.01 & 0.47 & 0.02 & 0.14 & 17.94 & 1.41 \\
\hline R15-11 & 46.62 & 0.01 & 34.72 & 0.00 & 0.50 & 0.00 & 0.13 & 18.25 & 1.35 \\
\hline R15-12 & 51.60 & 0.08 & 31.49 & 0.00 & 0.75 & 0.00 & 0.21 & 14.87 & 3.23 \\
\hline R15-12 & 51.45 & 0.02 & 30.88 & 0.00 & 0.78 & 0.00 & 0.24 & 13.89 & 3.46 \\
\hline R15-12 & 51.76 & 0.06 & 31.07 & 0.00 & 0.77 & 0.01 & 0.20 & 14.47 & 3.52 \\
\hline
\end{tabular}




$\begin{array}{llllllllll}\text { R15-14 } & 51.90 & 0.33 & 27.32 & 0.00 & 2.94 & 0.05 & 1.48 & 13.39 & 3.31 \\ \text { R15-14 } & 50.99 & 0.19 & 29.06 & 0.00 & 2.03 & 0.04 & 0.94 & 14.07 & 3.06 \\ \text { R15-13 } & 51.27 & 0.03 & 31.91 & 0.00 & 0.64 & 0.01 & 0.16 & 14.87 & 3.14 \\ \text { R15-13 } & 50.92 & 0.03 & 30.55 & 0.00 & 0.66 & 0.00 & 0.19 & 14.79 & 3.34 \\ \text { R15-13 } & 51.60 & 0.04 & 31.55 & 0.02 & 0.67 & 0.00 & 0.22 & 14.59 & 3.44 \\ \text { R15-15 } & 47.91 & 0.03 & 34.00 & 0.00 & 0.64 & 0.02 & 0.17 & 17.06 & 1.76 \\ \text { R15-15 } & 47.38 & 0.03 & 34.41 & 0.00 & 0.61 & 0.01 & 0.12 & 17.75 & 1.61 \\ \text { R15-15 } & 47.17 & 0.02 & 34.34 & 0.01 & 0.68 & 0.01 & 0.12 & 17.74 & 1.51 \\ \text { R15-15 } & 48.73 & 0.04 & 33.44 & 0.02 & 0.61 & 0.02 & 0.17 & 16.73 & 2.08 \\ \text { R15-15 } & 47.51 & 0.00 & 34.03 & 0.01 & 0.55 & 0.00 & 0.13 & 17.30 & 1.56 \\ \text { R15-15 } & 47.24 & 0.04 & 34.15 & 0.01 & 0.52 & 0.04 & 0.14 & 17.73 & 1.33 \\ \text { R15-15 } & 49.36 & 0.03 & 33.29 & 0.01 & 0.53 & 0.01 & 0.18 & 16.57 & 2.33 \\ \text { R15-16 } & 51.56 & 0.04 & 31.18 & 0.00 & 0.63 & 0.01 & 0.16 & 14.40 & 3.49 \\ \text { R15-16 } & 51.96 & 0.03 & 30.98 & 0.00 & 0.69 & 0.00 & 0.18 & 14.20 & 3.70\end{array}$




\begin{tabular}{rrr}
\multicolumn{1}{l}{ K2O } & \multicolumn{1}{l}{ P2O5 } & \multicolumn{1}{l}{ Total } \\
0.02 & 0.05 & 99.84 \\
0.03 & 0.07 & 100.02 \\
0.03 & 0.02 & 99.72 \\
0.03 & 0.03 & 99.82 \\
0.03 & 0.01 & 99.43 \\
0.03 & 0.00 & 98.91 \\
0.02 & 0.06 & 99.32 \\
0.03 & 0.01 & 99.27 \\
0.02 & 0.01 & 99.27 \\
0.03 & 0.01 & 99.38 \\
0.02 & 0.00 & 98.97 \\
0.02 & 0.05 & 98.51 \\
0.01 & 0.03 & 99.11 \\
0.03 & 0.01 & 99.15 \\
0.02 & 0.13 & 98.72 \\
0.03 & 0.10 & 99.14 \\
0.03 & 0.08 & 99.54 \\
0.02 & 0.02 & 99.19 \\
0.03 & 0.00 & 99.25 \\
0.03 & 0.16 & 98.68 \\
0.03 & 0.02 & 99.84 \\
0.02 & 0.03 & 99.45 \\
0.01 & 0.02 & 99.81 \\
0.04 & 0.00 & 98.47 \\
0.05 & 0.02 & 100.04 \\
0.04 & 0.02 & 100.12 \\
0.02 & 0.01 & 100.08 \\
0.03 & 0.04 & 99.04 \\
& &
\end{tabular}

$\begin{array}{ccc}\text { An mol\% } & \text { Ab mol\% } & \text { Or mol\% } \\ 82.6 & 17.2 & 0.1 \\ 75.0 & 24.8 & 0.2 \\ 72.3 & 27.5 & 0.2 \\ 72.4 & 27.4 & 0.2 \\ 78.2 & 21.6 & 0.2 \\ 71.3 & 28.6 & 0.2 \\ 78.4 & 21.5 & 0.1 \\ 78.1 & 21.7 & 0.2 \\ 78.8 & 21.1 & 0.1 \\ 73.6 & 26.3 & 0.2 \\ 75.9 & 24.0 & 0.1 \\ 74.4 & 25.5 & 0.1 \\ 72.0 & 28.0 & 0.1 \\ 74.9 & 24.9 & 0.2 \\ 72.6 & 27.3 & 0.1 \\ 73.4 & 26.5 & 0.2 \\ 73.6 & 26.2 & 0.2 \\ 74.9 & 25.0 & 0.1 \\ 74.8 & 25.1 & 0.2 \\ 75.6 & 24.2 & 0.2 \\ 74.0 & 25.8 & 0.2 \\ 82.4 & 17.5 & 0.1 \\ 74.4 & 25.6 & 0.1 \\ 70.0 & 29.7 & 0.2 \\ 68.0 & 31.7 & 0.3 \\ 66.9 & 32.8 & 0.2 \\ 77.0 & 22.9 & 0.1 \\ 74.6 & 25.2 & 0.2 \\ & & \end{array}$

\begin{tabular}{lrr}
\multicolumn{1}{l}{ K2O } & \multicolumn{1}{l}{ P2O5 } & \multicolumn{1}{l}{ Total } \\
0.03 & 0.03 & 98.62 \\
0.03 & 0.00 & 99.31 \\
0.04 & 0.00 & 99.12 \\
0.03 & 0.01 & 98.93 \\
0.04 & 0.00 & 98.88 \\
0.03 & 0.02 & 99.36 \\
0.03 & 0.00 & 98.19 \\
0.03 & 0.00 & 98.70 \\
0.02 & 0.01 & 98.30 \\
0.05 & 0.00 & 99.41 \\
0.04 & 0.02 & 98.56 \\
0.05 & 0.02 & 98.62 \\
0.02 & 0.00 & 98.54 \\
0.00 & 0.01 & 99.38 \\
0.00 & 0.04 & 98.27
\end{tabular}

An mol\% Ab mol\% Or mol\%

$\begin{array}{rrr}69.4 & 30.4 & 0.2 \\ 71.2 & 28.6 & 0.2 \\ 70.9 & 28.9 & 0.2 \\ 72.3 & 27.5 & 0.2 \\ 71.3 & 28.5 & 0.3 \\ 69.6 & 30.2 & 0.2 \\ 68.2 & 31.6 & 0.2 \\ 72.8 & 27.0 & 0.2 \\ 73.6 & 26.2 & 0.1 \\ 69.4 & 30.3 & 0.3 \\ 69.0 & 30.7 & 0.3 \\ 70.7 & 29.1 & 0.3 \\ 86.9 & 13.0 & 0.1 \\ 93.5 & 6.5 & 0.0 \\ 94.7 & 5.3 & 0.0\end{array}$




\begin{tabular}{|c|c|c|c|c|c|}
\hline 0.01 & 0.03 & 99.23 & 94.7 & 5.3 & 0.1 \\
\hline 0.00 & 0.01 & 99.31 & 93.6 & 6.4 & 0.0 \\
\hline 0.02 & 0.05 & 99.29 & 82.8 & 17.1 & 0.1 \\
\hline 0.02 & 0.02 & 99.52 & 86.2 & 13.7 & 0.1 \\
\hline 0.01 & 0.00 & 99.09 & 76.2 & 23.7 & 0.0 \\
\hline 0.03 & 0.01 & 99.20 & 75.5 & 24.3 & 0.2 \\
\hline 0.03 & 0.00 & 101.32 & 71.6 & 28.2 & 0.2 \\
\hline 0.03 & 0.00 & 101.05 & 71.5 & 28.3 & 0.2 \\
\hline 0.00 & 0.00 & 100.71 & 88.2 & 11.8 & 0.0 \\
\hline 0.01 & 0.00 & 101.39 & 87.8 & 12.2 & 0.0 \\
\hline 0.03 & 0.00 & 101.19 & 74.1 & 25.8 & 0.1 \\
\hline 0.02 & 0.00 & 100.96 & 74.8 & 25.0 & 0.1 \\
\hline $\mathrm{K} 2 \mathrm{O}$ & P2O5 & Total & An mol\% & $\mathrm{Ab} \mathrm{mol} \%$ & Or mol\% \\
\hline 0.02 & 0.01 & 99.68 & 79.2 & 20.7 & 0.1 \\
\hline 0.02 & 0.03 & 100.17 & 77.2 & 22.7 & 0.1 \\
\hline 0.01 & 0.01 & 99.51 & 78.8 & 21.1 & 0.1 \\
\hline 0.03 & 0.03 & 99.01 & 75.7 & 24.1 & 0.2 \\
\hline 0.01 & 0.01 & 99.27 & 79.3 & 20.6 & 0.1 \\
\hline 0.04 & 0.03 & 99.44 & 72.4 & 27.3 & 0.2 \\
\hline 0.03 & 0.11 & 99.50 & 75.4 & 24.4 & 0.2 \\
\hline 0.03 & 0.00 & 99.14 & 74.8 & 25.1 & 0.2 \\
\hline 0.02 & 0.02 & 99.30 & 77.0 & 22.8 & 0.1 \\
\hline 0.02 & 0.01 & 99.24 & 76.8 & 23.1 & 0.1 \\
\hline 0.03 & 0.02 & 98.48 & 77.0 & 22.8 & 0.2 \\
\hline 0.01 & 0.03 & 99.06 & 86.5 & 13.5 & 0.0 \\
\hline 0.03 & 0.02 & 98.45 & 75.3 & 24.5 & 0.2 \\
\hline 0.03 & 0.01 & 99.23 & 71.5 & 28.3 & 0.2 \\
\hline 0.04 & 0.02 & 98.58 & 67.6 & 32.1 & 0.2 \\
\hline 0.02 & 0.04 & 98.92 & 73.9 & 25.9 & 0.1 \\
\hline 0.04 & 0.05 & 98.70 & 70.7 & 29.0 & 0.3 \\
\hline 0.03 & 0.01 & 99.40 & 71.2 & 28.6 & 0.2 \\
\hline 0.04 & 0.00 & 99.13 & 68.5 & 31.3 & 0.2 \\
\hline 0.04 & 0.06 & 99.37 & 68.9 & 30.9 & 0.3 \\
\hline 0.01 & 0.03 & 99.31 & 84.9 & 15.0 & 0.1 \\
\hline 0.00 & 0.01 & 99.41 & 87.5 & 12.5 & 0.0 \\
\hline 0.09 & 0.05 & 98.27 & 73.3 & 26.0 & 0.6 \\
\hline 0.03 & 0.03 & 99.45 & 76.4 & 23.5 & 0.2 \\
\hline 0.02 & 0.04 & 100.88 & 85.8 & 14.1 & 0.1 \\
\hline 0.02 & 0.00 & 101.64 & 83.6 & 16.3 & 0.1 \\
\hline 0.00 & 0.00 & 101.68 & 86.7 & 13.3 & 0.0 \\
\hline 0.02 & 0.00 & 101.34 & 85.5 & 14.4 & 0.1 \\
\hline 0.01 & 0.00 & 101.20 & 87.5 & 12.4 & 0.1 \\
\hline 0.01 & 0.00 & 101.61 & 88.1 & 11.8 & 0.1 \\
\hline 0.04 & 0.00 & 102.30 & 71.6 & 28.1 & 0.2 \\
\hline 0.03 & 0.00 & 100.76 & 68.8 & 31.0 & 0.2 \\
\hline 0.02 & 0.00 & 101.93 & 69.4 & 30.5 & 0.1 \\
\hline
\end{tabular}




$\begin{array}{llllll}0.11 & 0.00 & 100.89 & 68.7 & 30.7 & 0.6 \\ 0.07 & 0.00 & 100.51 & 71.4 & 28.1 & 0.4 \\ 0.03 & 0.00 & 102.08 & 72.3 & 27.6 & 0.2 \\ 0.05 & 0.00 & 100.56 & 70.8 & 29.0 & 0.3 \\ 0.05 & 0.00 & 102.26 & 69.9 & 29.8 & 0.3 \\ 0.03 & 0.00 & 101.62 & 84.1 & 15.7 & 0.2 \\ 0.02 & 0.00 & 101.99 & 85.8 & 14.1 & 0.1 \\ 0.02 & 0.00 & 101.65 & 86.6 & 13.3 & 0.1 \\ 0.02 & 0.00 & 101.85 & 81.5 & 18.4 & 0.1 \\ 0.02 & 0.00 & 101.14 & 85.9 & 14.0 & 0.1 \\ 0.02 & 0.00 & 101.24 & 87.9 & 11.9 & 0.1 \\ 0.03 & 0.00 & 102.36 & 79.6 & 20.2 & 0.1 \\ 0.02 & 0.00 & 101.49 & 69.4 & 30.4 & 0.1 \\ 0.04 & 0.00 & 101.85 & 67.8 & 32.0 & 0.2\end{array}$




\begin{tabular}{|c|c|c|c|c|c|c|c|c|c|}
\hline & $\mathrm{SiO} 2$ & TiO2 & Al2O3 & $\mathrm{Cr} 2 \mathrm{O} 3$ & $\mathrm{FeO}$ & $\mathrm{MnO}$ & $\mathrm{MgO}$ & $\mathrm{CaO}$ & $\mathrm{Na} 2 \mathrm{O}$ \\
\hline R15-3 & 41.58 & 1.04 & 13.48 & 0.01 & 16.49 & 0.29 & 10.21 & 11.47 & 2.03 \\
\hline R15-3 & 41.65 & 1.06 & 13.24 & 0.00 & 16.27 & 0.30 & 10.05 & 11.33 & 2.07 \\
\hline R15-3 & 41.41 & 1.02 & 13.41 & 0.01 & 16.53 & 0.32 & 10.26 & 11.40 & 1.88 \\
\hline & $\mathrm{SiO} 2$ & TiO2 & Al2O3 & $\mathrm{Cr} 2 \mathrm{O} 3$ & $\mathrm{FeO}$ & $\mathrm{MnO}$ & $\mathrm{MgO}$ & $\mathrm{CaO}$ & $\mathrm{Na} 2 \mathrm{O}$ \\
\hline R15-3 & 0.02 & 45.77 & 1.92 & 0.01 & 49.59 & 1.88 & 0.13 & 0.12 & 0.04 \\
\hline R15-3 & 6.23 & 45.58 & 1.75 & 0.02 & 36.98 & 1.78 & 1.21 & 4.82 & 0.05 \\
\hline
\end{tabular}




\begin{tabular}{rrrrrr}
\multicolumn{1}{l}{ K2O } & \multicolumn{1}{c}{$\mathrm{P} 2 \mathrm{O} 5$} & $\mathrm{~F}$ & \multicolumn{2}{c}{$\mathrm{Cl}$} & \multicolumn{2}{c}{$\mathrm{SO3}$} & \multicolumn{1}{c}{ Total } \\
0.32 & 0.03 & 0.08 & 0.13 & 0.00 & 97.10 \\
0.33 & 0.01 & 0.04 & 0.11 & 0.00 & 96.42 \\
0.33 & 0.04 & 0.01 & 0.12 & 0.02 & 96.73 \\
& & & & & \\
K2O & P2O5 & \multicolumn{1}{c}{ Total } & & & \\
0.00 & 0.05 & 99.52 & & & \\
0.04 & 0.07 & 98.59 & & &
\end{tabular}


Supplementary Document 3. Electron microprobe analyses for matrix minerals and rock fragments

\begin{tabular}{|c|c|c|c|c|c|c|c|c|}
\hline olivine & $\mathrm{SiO} 2$ & $\mathrm{TiO} 2$ & $\mathrm{Al} 2 \mathrm{O} 3$ & $\mathrm{Cr} 2 \mathrm{O} 3$ & $\mathrm{FeO}$ & $\mathrm{MnO}$ & $\mathrm{MgO}$ & $\mathrm{CaO}$ \\
\hline $\mathrm{R} 05-14 \mathrm{~m} 2$ & 41.74 & 0.01 & 0.02 & 0.00 & 8.75 & 0.15 & 48.51 & 0.03 \\
\hline R05-19m3 & 41.89 & 0.04 & 0.04 & 0.00 & 8.86 & 0.12 & 48.41 & 0.06 \\
\hline R05-19m4 & 41.29 & 0.01 & 0.05 & 0.00 & 8.29 & 0.13 & 48.64 & 0.02 \\
\hline R05-19m16 & 41.88 & 0.00 & 0.03 & 0.01 & 8.01 & 0.11 & 49.82 & 0.04 \\
\hline orthopyroxene & $\mathrm{SiO} 2$ & $\mathrm{TiO} 2$ & Al2O3 & $\mathrm{Cr} 2 \mathrm{O} 3$ & $\mathrm{FeO}$ & $\mathrm{MnO}$ & $\mathrm{MgO}$ & $\mathrm{CaO}$ \\
\hline R05-13-m7 & 55.45 & 0.33 & 1.34 & 0.11 & 14.59 & 0.31 & 25.86 & 2.66 \\
\hline epidote & $\mathrm{SiO} 2$ & $\mathrm{TiO} 2$ & Al2O3 & $\mathrm{Cr} 2 \mathrm{O} 3$ & $\mathrm{FeO}$ & $\mathrm{MnO}$ & $\mathrm{MgO}$ & $\mathrm{CaO}$ \\
\hline R05-19m10 & 39.00 & 0.14 & 26.48 & 0.02 & 9.01 & 0.04 & 0.07 & 23.84 \\
\hline amphibole & $\mathrm{SiO} 2$ & $\mathrm{TiO} 2$ & Al2O3 & $\mathrm{Cr} 2 \mathrm{O} 3$ & $\mathrm{FeO}$ & $\mathrm{MnO}$ & $\mathrm{MgO}$ & $\mathrm{CaO}$ \\
\hline R05-13-m12 & 45.96 & 0.79 & 12.86 & 0.11 & 11.51 & 0.21 & 13.45 & 11.49 \\
\hline magnetite & $\mathrm{SiO} 2$ & $\mathrm{TiO} 2$ & $\mathrm{Al} 2 \mathrm{O} 3$ & $\mathrm{Cr} 2 \mathrm{O} 3$ & $\mathrm{FeO}$ & $\mathrm{MnO}$ & $\mathrm{MgO}$ & $\mathrm{CaO}$ \\
\hline R05-13-m8 & 0.25 & 4.03 & 2.03 & 0.03 & 87.12 & 0.56 & 1.28 & 0.02 \\
\hline R05-19-m1 & 0.12 & 7.10 & 5.35 & 0.87 & 77.50 & 0.35 & 4.18 & 0.02 \\
\hline R05-19m2 & 0.11 & 7.17 & 5.33 & 0.88 & 78.42 & 0.33 & 4.31 & 0.02 \\
\hline R05-19m20 & 0.16 & 0.02 & 0.08 & 0.00 & 94.01 & 0.12 & 0.28 & 0.04 \\
\hline clinopyroxene & $\mathrm{SiO} 2$ & $\mathrm{TiO} 2$ & Al2O3 & $\mathrm{Cr} 2 \mathrm{O} 3$ & $\mathrm{FeO}$ & $\mathrm{MnO}$ & $\mathrm{MgO}$ & $\mathrm{CaO}$ \\
\hline R05-13-mtx15 & 53.30 & 0.28 & 2.33 & 0.61 & 4.92 & 0.11 & 18.23 & 19.64 \\
\hline R05-13-mtx16 & 51.56 & 0.53 & 4.44 & 0.53 & 4.87 & 0.15 & 15.82 & 21.53 \\
\hline R05-14mtx6 & 52.15 & 0.46 & 3.39 & 0.73 & 4.62 & 0.09 & 15.94 & 22.13 \\
\hline R05-14mtx11 & 50.92 & 0.61 & 5.31 & 1.02 & 4.63 & 0.09 & 15.69 & 21.27 \\
\hline R05-18mtx1 & 49.62 & 1.09 & 5.24 & 0.04 & 8.09 & 0.23 & 15.36 & 18.75 \\
\hline R05-18mtx3 & 50.91 & 0.64 & 4.39 & 0.20 & 6.13 & 0.15 & 15.04 & 21.35 \\
\hline R05-18mtx4 & 51.93 & 0.54 & 3.47 & 0.09 & 6.33 & 0.19 & 16.09 & 20.92 \\
\hline R05-18mtx7 & 51.52 & 0.67 & 3.95 & 0.43 & 5.52 & 0.09 & 15.73 & 21.37 \\
\hline R05-18mtx8 & 50.79 & 0.71 & 4.74 & 0.45 & 5.81 & 0.08 & 15.85 & 20.77 \\
\hline R05-18mtx9 & 52.06 & 0.44 & 2.91 & 0.43 & 6.38 & 0.14 & 17.17 & 19.59 \\
\hline R05-18mtx10 & 51.50 & 0.68 & 4.37 & 0.41 & 6.23 & 0.13 & 15.73 & 20.78 \\
\hline R05-18mtx11 & 53.01 & 0.44 & 2.08 & 0.03 & 7.65 & 0.22 & 17.32 & 18.66 \\
\hline R05-18mtx12 & 49.96 & 1.25 & 5.37 & 0.11 & 7.04 & 0.17 & 14.43 & 21.06 \\
\hline R05-18mtx14 & 51.54 & 0.64 & 4.29 & 0.19 & 6.47 & 0.15 & 15.59 & 20.91 \\
\hline R05-18mtx15 & 52.42 & 0.58 & 3.67 & 0.13 & 6.04 & 0.14 & 15.23 & 21.34 \\
\hline R05-18mtx16 & 52.27 & 0.53 & 3.72 & 0.11 & 6.17 & 0.13 & 14.99 & 21.07 \\
\hline R05-18mtx18 & 53.31 & 0.31 & 1.77 & 0.31 & 6.14 & 0.18 & 17.95 & 19.39 \\
\hline R05-18mtx19 & 50.00 & 1.04 & 5.39 & 0.07 & 8.01 & 0.23 & 15.64 & 19.03 \\
\hline R05-18mtx20 & 52.04 & 0.63 & 4.01 & 0.13 & 6.40 & 0.15 & 15.98 & 20.31 \\
\hline R05-19mtx6 & 51.97 & 0.57 & 3.87 & 0.08 & 5.67 & 0.15 & 15.61 & 21.59 \\
\hline R05-19mtx7 & 54.07 & 0.30 & 2.11 & 0.27 & 5.07 & 0.17 & 17.81 & 20.39 \\
\hline R05-19mtx17 & 51.36 & 0.58 & 4.23 & 0.44 & 5.26 & 0.11 & 16.07 & 21.48 \\
\hline R05-19mtx18 & 51.26 & 0.55 & 3.94 & 0.62 & 4.50 & 0.08 & 15.67 & 22.52 \\
\hline R05-mtx1-e & 50.79 & 0.71 & 4.57 & 0.24 & 6.03 & 0.17 & 17.14 & 19.27 \\
\hline
\end{tabular}




\begin{tabular}{|c|c|c|c|c|c|c|c|c|}
\hline R05-mtx3-b & 49.85 & 0.59 & 5.04 & 0.08 & 6.78 & 0.16 & 15.72 & 19.94 \\
\hline R05-mtx3-d & 49.38 & 0.81 & 4.89 & 0.10 & 6.20 & 0.15 & 15.14 & 20.90 \\
\hline R05-19mtx8 & 52.20 & 0.46 & 1.48 & 0.00 & 15.09 & 0.69 & 12.25 & 18.34 \\
\hline plagioclase & $\mathrm{SiO} 2$ & $\mathrm{TiO} 2$ & $\mathrm{Al} 2 \mathrm{O} 3$ & $\mathrm{Cr} 2 \mathrm{O} 3$ & $\mathrm{FeO}$ & $\mathrm{MnO}$ & $\mathrm{MgO}$ & $\mathrm{CaO}$ \\
\hline R05-13-mtx3 & 47.61 & 0.01 & 33.78 & 0.01 & 0.59 & 0.00 & 0.17 & 17.44 \\
\hline R05-13-mtx18 & 49.57 & 0.03 & 32.76 & 0.00 & 0.61 & 0.08 & 0.22 & 15.88 \\
\hline R05-13-mtx19 & 49.12 & 0.00 & 33.08 & 0.00 & 0.76 & 0.05 & 0.04 & 16.17 \\
\hline R05-13-mtx21 & 51.42 & 0.06 & 30.86 & 0.02 & 1.04 & 0.00 & 0.22 & 14.58 \\
\hline R05-14mtx3 & 49.18 & 0.06 & 33.21 & 0.01 & 0.56 & 0.01 & 0.18 & 16.35 \\
\hline R05-14mtx4 & 50.54 & 0.03 & 31.60 & 0.02 & 0.60 & 0.01 & 0.20 & 15.13 \\
\hline R05-14mtx10 & 50.22 & 0.00 & 32.23 & 0.00 & 0.61 & 0.00 & 0.22 & 15.75 \\
\hline R05-14mtx12 & 45.55 & 0.00 & 35.44 & 0.02 & 0.52 & 0.00 & 0.14 & 18.77 \\
\hline R05-14mtx13 & 45.89 & 0.03 & 35.05 & 0.01 & 0.45 & 0.00 & 0.13 & 18.81 \\
\hline R05-14mtx14 & 48.57 & 0.04 & 32.70 & 0.00 & 0.67 & 0.00 & 0.20 & 16.21 \\
\hline R05-18mtx2 & 52.25 & 0.05 & 30.43 & 0.00 & 0.74 & 0.01 & 0.16 & 13.78 \\
\hline R05-18mtx5 & 49.53 & 0.00 & 32.15 & 0.00 & 0.61 & 0.00 & 0.19 & 15.70 \\
\hline R05-18mtx6 & 50.51 & 0.07 & 31.79 & 0.02 & 0.71 & 0.01 & 0.17 & 15.17 \\
\hline R05-18mtx13 & 52.43 & 0.04 & 30.67 & 0.00 & 0.72 & 0.01 & 0.19 & 13.86 \\
\hline R05-18mtx17 & 51.14 & 0.04 & 31.39 & 0.01 & 0.71 & 0.04 & 0.17 & 14.74 \\
\hline R05-19mtx12 & 49.75 & 0.02 & 32.28 & 0.01 & 0.65 & 0.02 & 0.20 & 15.56 \\
\hline R05-19mtx13 & 49.31 & 0.03 & 32.44 & 0.01 & 1.01 & 0.02 & 0.09 & 16.03 \\
\hline R05-19mtx15 & 50.61 & 0.01 & 31.08 & 0.01 & 1.03 & 0.02 & 0.11 & 14.86 \\
\hline R05-19mtx19 & 50.46 & 0.04 & 31.61 & 0.00 & 0.61 & 0.03 & 0.20 & 15.41 \\
\hline R05-mtx3-c & 50.33 & 0.02 & 30.97 & 0.01 & 0.60 & 0.01 & 0.17 & 13.83 \\
\hline R05-mtx1-b & 67.08 & 0.00 & 21.80 & 0.00 & 0.29 & 0.00 & 0.01 & 1.38 \\
\hline R05-13-mtx10 & 58.91 & 0.04 & 25.82 & 0.00 & 1.13 & 0.01 & 0.13 & 9.34 \\
\hline R05-13-mtx17 & 64.70 & 0.03 & 23.68 & 0.00 & 0.27 & 0.03 & 0.03 & 4.56 \\
\hline R05-19mtx5 & 60.11 & 0.03 & 26.54 & 0.00 & 0.39 & 0.02 & 0.02 & 8.24 \\
\hline glass & $\mathrm{SiO} 2$ & $\mathrm{TiO} 2$ & $\mathrm{Al} 2 \mathrm{O} 3$ & $\mathrm{Cr} 2 \mathrm{O} 3$ & $\mathrm{FeO}$ & $\mathrm{MnO}$ & $\mathrm{MgO}$ & $\mathrm{CaO}$ \\
\hline R05-13-mtx1 & 53.02 & 1.20 & 16.25 & 0.01 & 9.16 & 0.16 & 6.18 & 11.26 \\
\hline R05-13-mtx4 & 52.82 & 1.05 & 17.09 & 0.00 & 8.17 & 0.15 & 6.06 & 11.40 \\
\hline R05-14mtx1 & 51.96 & 1.16 & 16.57 & 0.02 & 8.52 & 0.18 & 6.05 & 11.46 \\
\hline R05-14mtx5 & 52.44 & 1.07 & 16.99 & 0.01 & 8.20 & 0.16 & 6.33 & 11.41 \\
\hline R05-14mtx7 & 53.13 & 1.17 & 17.40 & 0.03 & 8.44 & 0.16 & 4.57 & 10.00 \\
\hline R05-14mtx8 & 55.44 & 1.02 & 13.90 & 0.00 & 12.13 & 0.26 & 3.92 & 8.20 \\
\hline R05-14mtx9 & 53.50 & 1.15 & 16.41 & 0.02 & 9.28 & 0.19 & 5.94 & 11.16 \\
\hline R05-19mtx9 & 55.54 & 1.71 & 15.68 & 0.02 & 10.38 & 0.20 & 3.39 & 7.88 \\
\hline Hi-Si glass & $\mathrm{SiO} 2$ & $\mathrm{TiO} 2$ & $\mathrm{Al} 2 \mathrm{O} 3$ & $\mathrm{Cr} 2 \mathrm{O} 3$ & $\mathrm{FeO}$ & $\mathrm{MnO}$ & $\mathrm{MgO}$ & $\mathrm{CaO}$ \\
\hline R05-mtx19 & 70.54 & 0.76 & 12.21 & 0.01 & 5.22 & 0.09 & 0.75 & 2.95 \\
\hline
\end{tabular}

R06

olivine

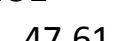

49.57

0.01

33.78

( 


\begin{tabular}{|c|c|c|c|c|c|c|c|c|}
\hline R06-mtx1-b & 37.43 & 0.02 & 0.07 & 0.00 & 19.40 & 0.29 & 40.46 & 0.28 \\
\hline serpentine & $\mathrm{SiO} 2$ & $\mathrm{TiO} 2$ & $\mathrm{Al} 2 \mathrm{O} 3$ & $\mathrm{Cr} 2 \mathrm{O} 3$ & $\mathrm{FeO}$ & $\mathrm{MnO}$ & $\mathrm{MgO}$ & $\mathrm{CaO}$ \\
\hline R06-mtx1-c & 42.20 & 0.03 & 0.63 & 0.01 & 1.84 & 0.03 & 38.86 & 0.07 \\
\hline quartz & $\mathrm{SiO} 2$ & TiO2 & Al2O3 & $\mathrm{Cr} 2 \mathrm{O} 3$ & $\mathrm{FeO}$ & $\mathrm{MnO}$ & $\mathrm{MgO}$ & $\mathrm{CaO}$ \\
\hline R06-16mtx1 & 98.53 & 0.01 & 0.19 & 0.01 & 0.35 & 0.00 & 0.15 & 0.03 \\
\hline clinopyroxene & $\mathrm{SiO} 2$ & $\mathrm{TiO} 2$ & $\mathrm{Al} 2 \mathrm{O} 3$ & $\mathrm{Cr} 2 \mathrm{O} 3$ & $\mathrm{FeO}$ & $\mathrm{MnO}$ & $\mathrm{MgO}$ & $\mathrm{CaO}$ \\
\hline R06-12mtx1 & 52.64 & 0.14 & 3.28 & 1.32 & 2.96 & 0.03 & 16.50 & 23.44 \\
\hline $\mathrm{R} 06-12 \mathrm{mt} \times 2$ & 52.05 & 0.50 & 4.18 & 0.18 & 5.45 & 0.12 & 16.90 & 20.34 \\
\hline R06-12mtx6 & 53.73 & 0.32 & 1.84 & 0.28 & 6.07 & 0.16 & 18.05 & 19.94 \\
\hline R06-13mtx2 & 54.16 & 0.28 & 2.09 & 0.28 & 4.81 & 0.11 & 17.59 & 21.25 \\
\hline R06-13mtx4 & 51.47 & 0.50 & 4.39 & 0.51 & 5.08 & 0.09 & 15.63 & 22.02 \\
\hline R06-mtx1-a & 49.66 & 0.72 & 4.72 & 0.54 & 5.91 & 0.12 & 15.94 & 19.96 \\
\hline R06-14mtx1 & 53.30 & 0.38 & 2.12 & 0.02 & 7.17 & 0.24 & 17.83 & 19.11 \\
\hline plagioclase & $\mathrm{SiO} 2$ & $\mathrm{TiO} 2$ & $\mathrm{Al} 2 \mathrm{O} 3$ & $\mathrm{Cr} 2 \mathrm{O} 3$ & $\mathrm{FeO}$ & $\mathrm{MnO}$ & $\mathrm{MgO}$ & $\mathrm{CaO}$ \\
\hline R06-12mtx3 & 50.67 & 0.04 & 32.12 & 0.00 & 0.65 & 0.00 & 0.21 & 14.93 \\
\hline R06-12mtx4 & 50.72 & 0.07 & 32.04 & 0.00 & 0.61 & 0.01 & 0.22 & 14.99 \\
\hline R06-13mtx1 & 49.39 & 0.01 & 32.92 & 0.01 & 0.62 & 0.00 & 0.19 & 15.98 \\
\hline R06-13mtx3 & 50.00 & 0.04 & 32.25 & 0.00 & 0.67 & 0.04 & 0.17 & 15.13 \\
\hline R06-16mtx3 & 47.11 & 0.02 & 34.39 & 0.00 & 0.84 & 0.01 & 0.16 & 17.35 \\
\hline R06-mtx2-b & 48.39 & 0.04 & 32.87 & 0.00 & 0.58 & 0.01 & 0.17 & 15.31 \\
\hline glass & $\mathrm{SiO} 2$ & TiO2 & Al2O3 & $\mathrm{Cr} 2 \mathrm{O} 3$ & $\mathrm{FeO}$ & $\mathrm{MnO}$ & $\mathrm{MgO}$ & $\mathrm{CaO}$ \\
\hline R06-12mtx7 & 53.56 & 1.18 & 17.38 & 0.01 & 8.69 & 0.15 & 4.38 & 9.74 \\
\hline R06-14mtx2 & 53.30 & 1.18 & 17.38 & 0.01 & 8.49 & 0.16 & 4.48 & 9.85 \\
\hline R06-16mtx4 & 51.37 & 1.14 & 16.90 & 0.01 & 8.98 & 0.18 & 4.00 & 9.64 \\
\hline R06-16mtx5 & 53.11 & 1.14 & 16.72 & 0.01 & 8.85 & 0.15 & 5.28 & 10.15 \\
\hline R06-mtx2-a & 49.95 & 0.90 & 18.00 & 0.02 & 7.82 & 0.15 & 5.66 & 10.87 \\
\hline R06-mtx2-d & 52.22 & 1.45 & 16.60 & 0.00 & 9.70 & 0.18 & 4.06 & 8.38 \\
\hline R06-mtx2-e & 52.42 & 1.46 & 16.80 & 0.01 & 9.57 & 0.16 & 3.90 & 8.30 \\
\hline R06-mtx2-f & 50.36 & 1.04 & 16.66 & 0.01 & 8.29 & 0.16 & 6.15 & 11.09 \\
\hline R06-mtx2-g & 50.17 & 1.09 & 16.64 & 0.00 & 8.34 & 0.15 & 6.16 & 10.83 \\
\hline
\end{tabular}

\section{R15}

olivine

R15-11mtx1

R15-11mtx2

R15-11mtx9

R15-11mtx21

R15-mtx1-a

$R 15-m t \times 3-c$

R15-mtx1-3

serpentine

\begin{tabular}{|c|c|c|c|c|c|c|c|}
\hline & TiO2 & $\mathrm{Al} 2 \mathrm{O} 3$ & $\mathrm{Cr} 2 \mathrm{O} 3$ & $\mathrm{FeO}$ & $\mathrm{MnO}$ & $\mathrm{MgO}$ & $\mathrm{CaO}$ \\
\hline 42.00 & 0.02 & 0.02 & 0.00 & 7.84 & 0.11 & 49.96 & 0.01 \\
\hline 42.07 & 0.03 & 0.01 & 0.01 & 7.80 & 0.15 & 50.07 & 0.01 \\
\hline 41.03 & 0.00 & 0.00 & 0.00 & 9.90 & 0.14 & 48.70 & 0.03 \\
\hline 41.29 & 0.00 & 0.01 & 0.03 & 8.22 & 0.10 & 48.89 & 0.02 \\
\hline 40.53 & 0.00 & 0.01 & 0.01 & 8.35 & 0.16 & 50.09 & 0.02 \\
\hline 41.63 & 0.03 & 0.01 & 0.00 & 9.17 & 0.16 & 50.90 & 0.03 \\
\hline 40.80 & 0.02 & 0.02 & 0.00 & 8.23 & 0.13 & 50.34 & 0.02 \\
\hline
\end{tabular}

$\begin{array}{llllllll}\mathrm{SiO} 2 & \mathrm{TiO} 2 & \mathrm{Al} 2 \mathrm{O} 3 & \mathrm{Cr} 2 \mathrm{O} 3 & \mathrm{FeO} & \mathrm{MnO} & \mathrm{MgO} & \mathrm{CaO}\end{array}$ 


\begin{tabular}{|c|c|c|c|c|c|c|c|c|}
\hline R15-mtx1-d & 42.55 & 0.03 & 1.02 & 0.03 & 4.07 & 0.06 & 36.90 & 0.14 \\
\hline R15-mtx2-a & 41.76 & 0.00 & 1.20 & 0.00 & 2.91 & 0.04 & 36.26 & 0.18 \\
\hline R15-mtx3-a & 41.99 & 0.02 & 0.87 & 0.01 & 4.38 & 0.04 & 36.07 & 0.13 \\
\hline R15-mtx1-7 & 40.39 & 0.05 & 1.39 & 0.15 & 7.02 & 0.09 & 31.53 & 0.04 \\
\hline R15-mtx3-2 & 42.34 & 0.05 & 1.18 & 0.00 & 2.51 & 0.05 & 37.39 & 0.11 \\
\hline R15-mtx11 & 41.82 & 0.06 & 1.35 & 0.02 & 3.53 & 0.1 & 35.45 & 0.44 \\
\hline epidote & $\mathrm{SiO} 2$ & $\mathrm{TiO} 2$ & $\mathrm{Al} 2 \mathrm{O} 3$ & $\mathrm{Cr} 2 \mathrm{O} 3$ & $\mathrm{FeO}$ & $\mathrm{MnO}$ & $\mathrm{MgO}$ & $\mathrm{CaO}$ \\
\hline R15-11mtx4 & 38.55 & 0.04 & 29.20 & 0.01 & 5.80 & 0.30 & 0.18 & 23.76 \\
\hline amphibole & $\mathrm{SiO} 2$ & TiO2 & $\mathrm{Al} 2 \mathrm{O} 3$ & $\mathrm{Cr} 2 \mathrm{O} 3$ & $\mathrm{FeO}$ & $\mathrm{MnO}$ & $\mathrm{MgO}$ & $\mathrm{CaO}$ \\
\hline R15-mtx1-c & 42.96 & 1.04 & 13.65 & 0.02 & 14.42 & 0.33 & 11.70 & 11.04 \\
\hline R15-gls11-32 & 44.10 & 0.15 & 14.21 & 0.06 & 7.10 & 0.13 & 16.06 & 11.81 \\
\hline clinopyroxene & $\mathrm{SiO} 2$ & TiO2 & Al2O3 & $\mathrm{Cr} 2 \mathrm{O} 3$ & $\mathrm{FeO}$ & $\mathrm{MnO}$ & $\mathrm{MgO}$ & $\mathrm{CaO}$ \\
\hline R15-11mtx5 & 51.80 & 0.67 & 1.83 & 0.04 & 10.90 & 0.35 & 13.15 & 21.02 \\
\hline R15-11mtx10 & 46.30 & 2.31 & 8.39 & 0.00 & 11.06 & 0.23 & 11.39 & 20.65 \\
\hline R15-11mtx11 & 45.76 & 2.30 & 8.26 & 0.03 & 13.36 & 0.28 & 10.99 & 19.26 \\
\hline R15-11mtx14 & 43.05 & 3.40 & 9.32 & 0.02 & 14.04 & 0.28 & 9.53 & 20.04 \\
\hline R15-11mtx15 & 46.03 & 2.38 & 8.62 & 0.01 & 11.01 & 0.23 & 11.75 & 19.40 \\
\hline R15-11mtx16 & 47.84 & 2.06 & 7.12 & 0.03 & 11.07 & 0.20 & 13.03 & 19.33 \\
\hline R15-11mtx17 & 44.88 & 2.65 & 9.45 & 0.03 & 11.83 & 0.21 & 10.38 & 20.49 \\
\hline R15-11mtx18 & 48.40 & 1.80 & 6.22 & 0.03 & 10.08 & 0.23 & 13.08 & 20.49 \\
\hline plagioclase & $\mathrm{SiO} 2$ & $\mathrm{TiO} 2$ & $\mathrm{Al} 2 \mathrm{O3}$ & $\mathrm{Cr} 2 \mathrm{O} 3$ & $\mathrm{FeO}$ & $\mathrm{MnO}$ & $\mathrm{MgO}$ & $\mathrm{CaO}$ \\
\hline R15-11mtx19 & 51.43 & 0.08 & 31.15 & 0.00 & 0.91 & 0.02 & 0.24 & 14.71 \\
\hline R15-11mtx20 & 49.70 & 0.03 & 32.89 & 0.00 & 0.84 & 0.02 & 0.00 & 15.40 \\
\hline R15-mtx1-e & 47.40 & 0.02 & 33.56 & 0.02 & 0.55 & 0.02 & 0.13 & 16.17 \\
\hline R15-mtx3-1 & 45.90 & 0.04 & 33.75 & 0.00 & 0.83 & 0.01 & 0.07 & 16.82 \\
\hline R15-gls11-28 & 49.90 & 0.06 & 31.65 & 0.05 & 0.83 & 0.00 & 0.00 & 14.94 \\
\hline R15-gls11-31 & 49.21 & 0.02 & 32.26 & 0.00 & 0.55 & 0.00 & 0.19 & 15.61 \\
\hline R15-11mtx8 & 59.45 & 0.07 & 25.94 & 0.02 & 1.03 & 0.02 & 0.06 & 8.77 \\
\hline $\mathrm{R} 15-11 \mathrm{mtx} 13$ & 55.60 & 0.11 & 27.88 & 0.00 & 1.36 & 0.00 & 0.20 & 11.63 \\
\hline glass & $\mathrm{SiO} 2$ & $\mathrm{TiO} 2$ & $\mathrm{Al} 2 \mathrm{O} 3$ & $\mathrm{Cr} 2 \mathrm{O} 3$ & $\mathrm{FeO}$ & $\mathrm{MnO}$ & $\mathrm{MgO}$ & $\mathrm{CaO}$ \\
\hline R15-11mtx7 & 52.66 & 1.11 & 16.85 & 0.00 & 8.59 & 0.17 & 6.01 & 10.87 \\
\hline R15-mtx1-f & 52.32 & 1.14 & 17.55 & 0.02 & 8.88 & 0.17 & 4.71 & 9.48 \\
\hline R15-mtx2-b & 50.58 & 1.03 & 16.82 & 0.01 & 7.89 & 0.15 & 6.51 & 10.93 \\
\hline R15-mtx2-b & 50.95 & 0.99 & 16.79 & 0.02 & 7.79 & 0.14 & 6.76 & 11.26 \\
\hline R15-mtx3-b & 51.11 & 1.08 & 17.86 & 0.00 & 8.32 & 0.15 & 4.90 & 9.62 \\
\hline R15-mtx2-1 & 50.21 & 1.03 & 16.85 & 0.01 & 7.75 & 0.14 & 6.71 & 11.11 \\
\hline R15-11 - 3 & 51.88 & 1.11 & 16.51 & 0.00 & 8.88 & 0.17 & 5.28 & 10.18 \\
\hline $\mathrm{R} 15-11 \mathrm{mt} \times 23$ & 53.37 & 1.14 & 16.41 & 0.00 & 8.74 & 0.19 & 5.14 & 9.58 \\
\hline R15-gls11-27 & 51.27 & 1.14 & 16.61 & 0.01 & 8.62 & 0.13 & 4.27 & 9.32 \\
\hline R15-gls11-30 & 50.35 & 1.08 & 16.43 & 0.00 & 8.40 & 0.14 & 5.09 & 9.98 \\
\hline
\end{tabular}




$\begin{array}{lrrrrrrrrr}\text { R15-11mtx3 } & 52.91 & 0.14 & 4.93 & 0.06 & 8.69 & 0.22 & 16.62 & 12.73 \\ \text { R15-11mtx6 } & 50.67 & 0.93 & 6.21 & 0.14 & 11.22 & 0.14 & 15.42 & 12.38 \\ & & & & & & & & & \\ & & & & & & & & & \\ \text { hi-Si glass } & \text { SiO2 } & \text { TiO2 } & \text { Al2O3 } & \text { Cr2O3 } & \text { FeO } & \text { MnO } & \text { MgO } & \mathrm{CaO} \\ \text { R15-11mtx21 } & 70.27 & 0.75 & 13.32 & 0 & 5.83 & 0.14 & 0.93 & 3.58\end{array}$




\begin{tabular}{|c|c|c|c|c|c|c|c|c|}
\hline $\mathrm{Na} 2 \mathrm{O}$ & $\mathrm{K} 2 \mathrm{O}$ & P2O5 & Total & & Fo & $\mathrm{Fa}$ & & \\
\hline 0.00 & 0.02 & 0.02 & 99.25 & & 90.7 & 9.2 & 0.2 & \\
\hline 0.00 & 0.01 & 0.02 & 99.51 & & 90.6 & 9.3 & 0.1 & \\
\hline 0.01 & 0.00 & 0.02 & 98.48 & & 91.1 & 8.7 & 0.1 & \\
\hline 0.00 & 0.00 & 0.00 & 99.90 & & 91.6 & 8.3 & 0.1 & \\
\hline $\mathrm{Na} 2 \mathrm{O}$ & $\mathrm{K} 2 \mathrm{O}$ & P2O5 & Total & & Wo & En & $\mathrm{Ac}$ & \\
\hline 0.04 & 0.01 & 0 & 100.72 & & 5.3 & 71.4 & 23.2 & 0.2 \\
\hline $\mathrm{Na} 2 \mathrm{O}$ & $\mathrm{K} 2 \mathrm{O}$ & P2O5 & $\mathrm{F}$ & $\mathrm{Cl}$ & $\mathrm{SO} 3$ & Total & & \\
\hline 0.00 & 0.01 & 0.04 & 0.0882 & 0.0197 & 0.0119 & 98.7325 & & \\
\hline $\mathrm{Na} 2 \mathrm{O}$ & $\mathrm{K} 2 \mathrm{O}$ & P2O5 & $\mathrm{F}$ & $\mathrm{Cl}$ & $\mathrm{SO} 3$ & Total & & \\
\hline 1.76 & 0.10 & 0.04 & 0.03 & 0.04 & 0.00 & 98.34 & & \\
\hline $\mathrm{Na} 2 \mathrm{O}$ & $\mathrm{K} 2 \mathrm{O}$ & P2O5 & $\mathrm{F}$ & $\mathrm{Cl}$ & $\mathrm{SO} 3$ & Total & & \\
\hline 0.00 & 0.00 & 0.05 & 0.00 & 0.00 & 0.00 & 95.38 & & \\
\hline 0.01 & 0.01 & 0.00 & 0.00 & 0.00 & 0.00 & 95.51 & & \\
\hline 0.00 & 0.01 & 0.02 & 0.00 & 0.01 & 0.00 & 96.61 & & \\
\hline 0.00 & 0.00 & 0.04 & 0.00 & 0.01 & 0.00 & 94.76 & & \\
\hline $\mathrm{Na} 2 \mathrm{O}$ & $\mathrm{K} 2 \mathrm{O}$ & P2O5 & Total & & Wo & En & $A C$ & \\
\hline 0.17 & 0.01 & 0.01 & 99.61 & & 39.9 & 51.5 & 8.0 & 0.6 \\
\hline 0.23 & 0.01 & 0.01 & 99.67 & & 45.0 & 46.0 & 8.2 & 0.9 \\
\hline 0.25 & 0.00 & 0.02 & 99.81 & & 45.7 & 45.8 & 7.6 & 0.9 \\
\hline 0.24 & 0.00 & 0.01 & 99.78 & & 45.0 & 46.2 & 7.8 & 0.9 \\
\hline 0.30 & 0.00 & 0.04 & 98.77 & & 39.8 & 45.3 & 13.7 & 1.1 \\
\hline 0.25 & 0.00 & 0.02 & 99.08 & & 44.8 & 43.9 & 10.3 & 1.0 \\
\hline 0.23 & 0.01 & 0.02 & 99.92 & & 42.9 & 45.9 & 10.4 & 0.9 \\
\hline 0.25 & 0.00 & 0.00 & 99.55 & & 44.4 & 45.5 & 9.1 & 0.9 \\
\hline 0.24 & 0.01 & 0.02 & 99.48 & & 43.4 & 46.1 & 9.6 & 0.9 \\
\hline 0.20 & 0.00 & 0.00 & 99.33 & & 40.0 & 48.8 & 10.4 & 0.7 \\
\hline 0.24 & 0.00 & 0.00 & 100.09 & & 43.2 & 45.5 & 10.3 & 0.9 \\
\hline 0.14 & 0.00 & 0.01 & 99.58 & & 38.0 & 49.0 & 12.5 & 0.5 \\
\hline 0.28 & 0.01 & 0.03 & 99.70 & & 44.6 & 42.5 & 11.9 & 1.1 \\
\hline 0.27 & 0.00 & 0.04 & 100.17 & & 43.3 & 44.9 & 10.7 & 1.0 \\
\hline 0.28 & 0.01 & 0.02 & 99.86 & & 44.6 & 44.3 & 10.1 & 1.1 \\
\hline 0.30 & 0.00 & 0.02 & 99.32 & & 44.4 & 44.0 & 10.4 & 1.1 \\
\hline 0.16 & 0.00 & 0.01 & 99.53 & & 39.1 & 50.4 & 9.9 & 0.6 \\
\hline 0.26 & 0.01 & 0.03 & 99.74 & & 39.9 & 45.7 & 13.5 & 1.0 \\
\hline 0.24 & 0.00 & 0.00 & 99.92 & & 42.2 & 46.2 & 10.6 & 0.9 \\
\hline 0.25 & 0.01 & 0.00 & 99.79 & & 44.7 & 45.0 & 9.4 & 0.9 \\
\hline 0.22 & 0.01 & 0.04 & 100.54 & & 41.0 & 49.9 & 8.3 & 0.8 \\
\hline 0.23 & 0.00 & 0.00 & 99.76 & & 44.3 & 46.2 & 8.6 & 0.8 \\
\hline 0.20 & 0.01 & 0.04 & 99.39 & & 46.7 & 45.2 & 7.4 & 0.7 \\
\hline 0.24 & 0.00 & 0.03 & 99.20 & & 39.8 & 49.3 & 10.0 & 0.9 \\
\hline
\end{tabular}




$\begin{array}{rrrrrrrr}0.34 & 0.02 & 0.08 & 98.65 & 41.7 & 45.7 & 11.3 & 1.3 \\ 0.27 & 0.01 & 0.02 & 97.94 & 44.1 & 44.4 & 10.4 & 1.0 \\ 0.24 & 0.01 & 0.01 & 100.78 & 38.1 & 35.4 & 25.6 & 0.9\end{array}$

\begin{tabular}{rrrrrrr} 
Na2O & \multicolumn{1}{l}{ K2O } & P2O5 & \multicolumn{1}{c}{ Total } & An mol\% & Ab mol\% & Or mol\% \\
1.80 & 0.02 & 0.03 & 101.48 & 84.1 & 15.7 & 0.1 \\
2.42 & 0.03 & 0.00 & 101.63 & 78.2 & 21.6 & 0.2 \\
2.30 & 0.06 & 0.03 & 101.66 & 79.2 & 20.4 & 0.4 \\
3.21 & 0.08 & 0.00 & 101.50 & 71.2 & 28.4 & 0.5 \\
2.44 & 0.03 & 0.03 & 102.07 & 78.6 & 21.2 & 0.2 \\
2.88 & 0.04 & 0.00 & 101.14 & 74.2 & 25.6 & 0.2 \\
2.55 & 0.03 & 0.03 & 101.64 & 77.2 & 22.6 & 0.2 \\
0.91 & 0.00 & 0.04 & 101.39 & 91.9 & 8.1 & 0.0 \\
0.94 & 0.00 & 0.00 & 101.32 & 91.7 & 8.3 & 0.0 \\
2.30 & 0.03 & 0.02 & 100.76 & 79.4 & 20.4 & 0.2 \\
3.68 & 0.03 & 0.05 & 101.21 & 67.3 & 32.5 & 0.2 \\
2.56 & 0.01 & 0.01 & 100.77 & 77.1 & 22.8 & 0.1 \\
3.00 & 0.03 & 0.07 & 101.61 & 73.5 & 26.3 & 0.2 \\
3.53 & 0.03 & 0.00 & 101.49 & 68.3 & 31.5 & 0.2 \\
3.28 & 0.02 & 0.03 & 101.59 & 71.2 & 28.7 & 0.1 \\
2.52 & 0.03 & 0.04 & 101.12 & 77.2 & 22.7 & 0.2 \\
2.18 & 0.12 & 0.02 & 101.40 & 79.7 & 19.6 & 0.7 \\
2.70 & 0.16 & 0.03 & 100.62 & 74.5 & 24.5 & 1.0 \\
2.91 & 0.04 & 0.05 & 101.35 & 74.4 & 25.4 & 0.2 \\
3.19 & 0.04 & 0.04 & 99.23 & 70.4 & 29.4 & 0.2 \\
10.92 & 0.09 & 0.06 & 101.65 & & & \\
5.15 & 0.29 & 0.02 & 100.93 & 6.5 & 93.0 & 0.5 \\
8.91 & 0.06 & 0.00 & 102.30 & 49.1 & 49.0 & 1.8 \\
6.71 & 0.02 & 0.02 & 102.20 & 22.0 & 77.7 & 0.4 \\
& & & & 40.4 & 59.5 & 0.1
\end{tabular}

\begin{tabular}{|c|c|c|c|c|c|c|}
\hline $\mathrm{Na} 2 \mathrm{O}$ & $\mathrm{K} 2 \mathrm{O}$ & P2O5 & $\mathrm{F}$ & $\mathrm{Cl}$ & $\mathrm{SO} 3$ & Total \\
\hline 0.47 & 0.16 & 0.12 & 0.00 & 0.08 & 0.20 & 98.25 \\
\hline 2.00 & 0.24 & 0.14 & 0.00 & 0.08 & 0.20 & 99.39 \\
\hline 1.04 & 0.22 & 0.10 & 0.00 & 0.17 & 0.12 & 97.54 \\
\hline 1.98 & 0.21 & 0.11 & 0.09 & 0.10 & 0.22 & 99.26 \\
\hline 0.70 & 0.13 & 0.17 & 0.00 & 0.21 & 0.10 & 96.18 \\
\hline 2.62 & 1.10 & 0.36 & 0.00 & 0.20 & 0.13 & 99.23 \\
\hline 0.37 & 0.16 & 0.11 & 0.05 & 0.07 & 0.22 & 98.59 \\
\hline 0.80 & 0.29 & 0.18 & 0.00 & 0.23 & 0.27 & 96.51 \\
\hline
\end{tabular}

\begin{tabular}{rrrrrrrrrr}
$\mathrm{Na} 2 \mathrm{O}$ & $\mathrm{K} 2 \mathrm{O}$ & $\mathrm{P} 2 \mathrm{O} 5$ & $\mathrm{~F}$ & & $\mathrm{Cl}$ & & $\mathrm{SO} 3$ & \multicolumn{2}{c}{ Total } \\
1.98 & 1.86 & 0.23 & & 0.07 & & 0.23 & & 0 & 96.83
\end{tabular}

\begin{tabular}{rrrrrrrr}
$\mathrm{Na} 2 \mathrm{O}$ & $\mathrm{K} 2 \mathrm{O}$ & \multicolumn{1}{c}{$\mathrm{P} 2 \mathrm{O} 5$} & Total & Fo & \multicolumn{2}{c}{ Fa } & Tp \\
0.00 & 0.01 & 0.11 & 100.19 & & 81.3 & 18.4 & 0.3
\end{tabular}




\begin{tabular}{|c|c|c|c|c|c|c|c|c|}
\hline 0.02 & 0.00 & 0.11 & 98.10 & & 78.5 & 21.1 & 0.3 & \\
\hline $\mathrm{Na} 2 \mathrm{O}$ & $\mathrm{K} 2 \mathrm{O}$ & P2O5 & $\mathrm{F}$ & $\mathrm{Cl}$ & SO3 & Total & & \\
\hline 0.04 & 0.03 & 0.02 & 0.06 & 0.03 & 0.06 & 83.88 & & \\
\hline $\mathrm{Na} 2 \mathrm{O}$ & $\mathrm{K} 2 \mathrm{O}$ & P2O5 & Total & & & & & \\
\hline 0.00 & 0.02 & 0.00 & 99.30 & & & & & \\
\hline $\mathrm{Na} 2 \mathrm{O}$ & $\mathrm{K} 2 \mathrm{O}$ & P2O5 & Total & & Wo & En & Ac & \\
\hline 0.25 & 0.02 & 0.00 & 100.59 & & 47.7 & 46.7 & 4.7 & 0.9 \\
\hline 0.19 & 0.00 & 0.03 & 99.95 & & 41.9 & 48.4 & 9.0 & 0.7 \\
\hline 0.17 & 0.00 & 0.03 & 100.63 & & 39.7 & 50.0 & 9.7 & 0.6 \\
\hline 0.18 & 0.01 & 0.01 & 100.80 & & 42.6 & 49.0 & 7.7 & 0.7 \\
\hline 0.23 & 0.01 & 0.02 & 99.98 & & 45.7 & 45.1 & 8.4 & 0.9 \\
\hline 0.26 & 0.00 & 0.07 & 97.92 & & 42.2 & 46.9 & 9.9 & 1.0 \\
\hline 0.17 & 0.00 & 0.03 & 100.39 & & 38.2 & 49.6 & 11.6 & 0.6 \\
\hline
\end{tabular}

\begin{tabular}{rrrr}
\multicolumn{1}{c}{$\mathrm{Na2O}$} & \multicolumn{1}{l}{ K2O } & \multicolumn{1}{l}{ P2O5 } & \multicolumn{1}{l}{ Total } \\
3.00 & 0.02 & 0.02 & 101.73 \\
2.99 & 0.03 & 0.04 & 101.75 \\
2.54 & 0.02 & 0.00 & 101.69 \\
2.76 & 0.03 & 0.02 & 101.15 \\
1.53 & 0.01 & 0.00 & 101.49 \\
2.45 & 0.02 & 0.05 & 99.89
\end{tabular}

An $\mathrm{mol} \% \quad$ Ab mol\% $\quad$ Or mol\%

$\begin{array}{lll}73.2 & 26.7 & 0.1 \\ 73.4 & 26.5 & 0.2 \\ 77.5 & 22.3 & 0.1 \\ 75.1 & 24.8 & 0.2 \\ 86.2 & 13.8 & 0.1 \\ 77.5 & 22.4 & 0.1\end{array}$

\begin{tabular}{|c|c|c|c|c|c|c|}
\hline $\mathrm{Na} 2 \mathrm{O}$ & $\mathrm{K} 2 \mathrm{O}$ & P2O5 & $F$ & $\mathrm{Cl}$ & $\mathrm{SO} 3$ & Total \\
\hline 0.97 & 0.27 & 0.15 & 0.00 & 0.19 & 0.24 & 96.87 \\
\hline 1.03 & 0.25 & 0.16 & 0.00 & 0.19 & 0.22 & 96.66 \\
\hline 2.05 & 0.26 & 0.14 & 0.00 & 0.19 & 0.20 & 95.00 \\
\hline 1.33 & 0.27 & 0.10 & 0.00 & 0.13 & 0.21 & 97.43 \\
\hline 2.35 & 0.25 & 0.14 & 0.00 & 0.12 & 0.13 & 96.34 \\
\hline 1.98 & 0.29 & 0.21 & 0.00 & 0.17 & 0.30 & 95.49 \\
\hline 2.10 & 0.28 & 0.18 & 0.04 & 0.20 & 0.33 & 95.69 \\
\hline 2.75 & 0.18 & 0.11 & 0.13 & 0.09 & 0.29 & 97.23 \\
\hline 2.60 & 0.18 & 0.14 & 0.00 & 0.09 & 0.29 & 96.65 \\
\hline
\end{tabular}

\begin{tabular}{rrrrrrr} 
Na2O & \multicolumn{1}{c}{ K2O } & P2O5 & \multicolumn{1}{c}{ Total } & Fo & Fa & Tp \\
0.00 & 0.00 & 0.02 & 100.00 & 91.8 & 8.1 & 0.1 \\
0.00 & 0.01 & 0.00 & 100.16 & 91.8 & 8.0 & 0.2 \\
0.00 & 0.01 & 0.02 & 99.82 & 89.6 & 10.2 & 0.1 \\
0.00 & 0.00 & 0.01 & 98.59 & 91.3 & 8.6 & 0.1 \\
0.01 & 0.00 & 0.01 & 99.19 & 91.3 & 8.5 & 0.2 \\
0.00 & 0.01 & 0.00 & 101.94 & 90.7 & 9.2 & 0.2 \\
0.00 & 0.00 & 0.01 & 99.60 & 91.5 & 8.4 & 0.1
\end{tabular}

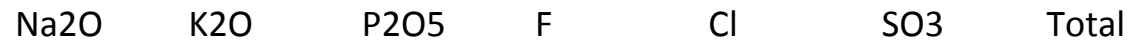




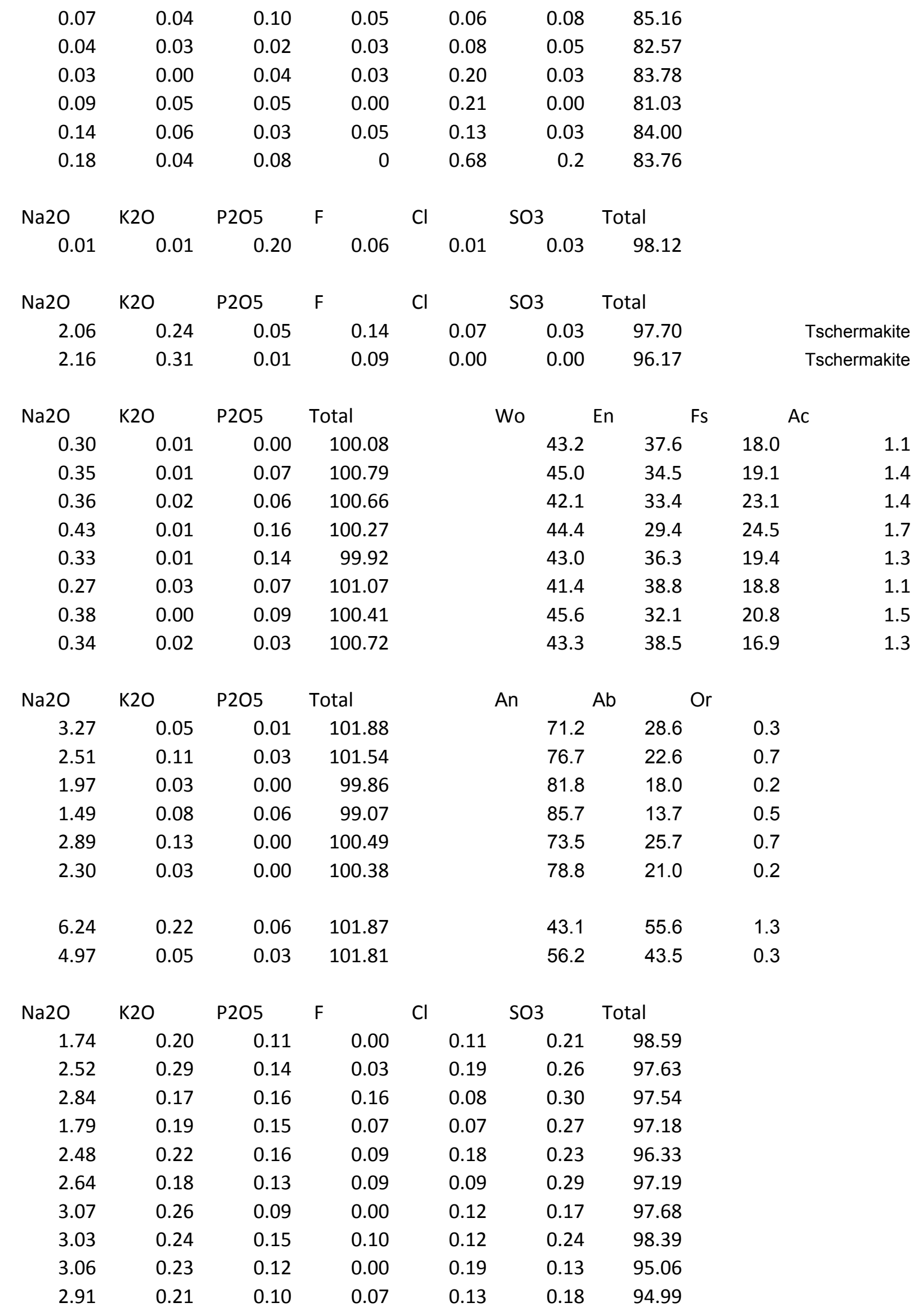




$\begin{array}{lllllll}0.57 & 0.02 & 0.01 & 0.00 & 0.02 & 0.01 & 96.92 \\ 1.17 & 0.04 & 0.00 & 0.06 & 0.07 & 0.01 & 98.41\end{array}$

\begin{tabular}{rrrrrrrrr}
$\mathrm{Na} 2 \mathrm{O}$ & $\mathrm{K} 2 \mathrm{O}$ & $\mathrm{P} 2 \mathrm{O} 5$ & $\mathrm{~F}$ & & $\mathrm{Cl}$ & \multicolumn{2}{c}{$\mathrm{SO} 3$} & \multicolumn{1}{c}{ Total } \\
0.95 & 1.17 & 0.09 & & 0 & & 0.3 & 0.03 & 97.31
\end{tabular}


Supplementary 1. Electron microprobe glass analyses

\begin{tabular}{|c|c|c|c|c|c|c|c|c|}
\hline R05 & $\mathrm{SiO} 2$ & TiO2 & Al2O3 & $\mathrm{Cr} 2 \mathrm{O} 3$ & $\mathrm{FeO}$ & $\mathrm{MnO}$ & $\mathrm{MgO}$ & $\mathrm{CaO}$ \\
\hline R05-12r & 50.89 & 1.00 & 16.60 & 0.00 & 8.21 & 0.16 & 6.43 & 11.50 \\
\hline R05-12r & 50.96 & 1.03 & 16.53 & 0.00 & 8.13 & 0.13 & 6.49 & 11.38 \\
\hline R05-12r & 50.44 & 0.97 & 16.51 & 0.00 & 8.00 & 0.15 & 6.43 & 11.45 \\
\hline R05-12r & 50.52 & 0.96 & 15.76 & 0.02 & 8.16 & 0.15 & 6.39 & 11.26 \\
\hline R05-12r & 50.92 & 0.99 & 16.52 & 0.02 & 8.18 & 0.16 & 6.34 & 11.28 \\
\hline R05-12r & 50.90 & 0.95 & 16.53 & 0.02 & 8.01 & 0.13 & 6.37 & 11.30 \\
\hline R05-12r & 50.89 & 0.99 & 16.48 & 0.03 & 7.96 & 0.13 & 6.36 & 11.36 \\
\hline R05-12r & 51.02 & 0.98 & 16.55 & 0.00 & 8.13 & 0.16 & 6.38 & 11.38 \\
\hline R05-12r & 50.81 & 0.99 & 16.56 & 0.00 & 8.19 & 0.16 & 6.14 & 11.24 \\
\hline R05-12r & 50.92 & 0.95 & 16.49 & 0.00 & 8.09 & 0.18 & 6.36 & 11.17 \\
\hline R05-12r & 50.63 & 0.99 & 16.57 & 0.03 & 7.87 & 0.15 & 6.42 & 11.28 \\
\hline R05-12r & 50.77 & 0.96 & 16.27 & 0.02 & 8.14 & 0.13 & 6.12 & 11.33 \\
\hline R05-12r & 50.82 & 1.00 & 16.60 & 0.01 & 8.18 & 0.16 & 6.37 & 11.34 \\
\hline R05-12r & 50.88 & 1.00 & 16.06 & 0.00 & 8.11 & 0.12 & 6.87 & 11.85 \\
\hline R05-12r & 50.83 & 1.02 & 16.51 & 0.01 & 8.18 & 0.15 & 6.43 & 11.33 \\
\hline R05-12r & 50.81 & 0.97 & 16.49 & 0.01 & 7.93 & 0.16 & 6.35 & 11.37 \\
\hline R05-12r & 50.96 & 0.99 & 16.56 & 0.01 & 8.20 & 0.16 & 6.18 & 11.32 \\
\hline R05-12r & 50.92 & 0.99 & 16.53 & 0.01 & 8.12 & 0.13 & 6.37 & 11.23 \\
\hline R05-12r & 50.80 & 0.94 & 16.49 & 0.00 & 8.12 & 0.15 & 6.31 & 11.21 \\
\hline R05-12r & 50.84 & 0.97 & 16.49 & 0.02 & 8.18 & 0.13 & 6.42 & 11.33 \\
\hline R05-12r & 50.94 & 1.00 & 16.42 & 0.01 & 8.14 & 0.16 & 6.36 & 11.15 \\
\hline R05-12r & 50.68 & 1.01 & 16.43 & 0.01 & 7.94 & 0.16 & 6.33 & 11.07 \\
\hline R05-12r & 50.92 & 1.00 & 16.57 & 0.00 & 8.21 & 0.14 & 6.39 & 11.30 \\
\hline R05-12r & 50.81 & 0.96 & 16.45 & 0.00 & 8.04 & 0.15 & 6.18 & 11.37 \\
\hline R05-12r & 51.06 & 0.99 & 16.53 & 0.03 & 8.18 & 0.16 & 6.33 & 11.42 \\
\hline R05-12r & 50.94 & 0.98 & 16.52 & 0.01 & 8.16 & 0.12 & 6.34 & 11.15 \\
\hline R05-12r & 50.91 & 1.00 & 16.40 & 0.01 & 7.99 & 0.15 & 6.27 & 11.45 \\
\hline R05-12r & 51.09 & 0.96 & 16.41 & 0.01 & 8.06 & 0.16 & 6.31 & 11.27 \\
\hline R05-12r & 51.01 & 0.97 & 16.58 & 0.02 & 8.14 & 0.17 & 6.34 & 11.35 \\
\hline R05-12r & 51.04 & 1.02 & 16.61 & 0.00 & 8.11 & 0.14 & 6.40 & 11.43 \\
\hline R05-12r & 50.85 & 0.98 & 16.53 & 0.01 & 8.14 & 0.11 & 6.25 & 11.29 \\
\hline R05-12r & 51.18 & 0.93 & 16.61 & 0.02 & 8.24 & 0.18 & 6.38 & 11.26 \\
\hline R05-12r & 50.69 & 0.98 & 16.51 & 0.00 & 8.10 & 0.14 & 6.29 & 11.34 \\
\hline R05-12r & 50.98 & 0.97 & 16.48 & 0.02 & 8.16 & 0.16 & 6.34 & 11.21 \\
\hline R05-12r & 51.09 & 0.95 & 16.52 & 0.01 & 8.07 & 0.12 & 6.31 & 11.03 \\
\hline R05-13r & 50.68 & 0.97 & 16.36 & 0.00 & 8.15 & 0.16 & 6.30 & 11.39 \\
\hline R05-13r & 50.64 & 0.99 & 16.11 & 0.02 & 8.04 & 0.15 & 6.31 & 11.33 \\
\hline R05-13r & 50.92 & 0.97 & 16.63 & 0.00 & 8.30 & 0.15 & 6.35 & 11.35 \\
\hline R05-13r & 50.24 & 1.04 & 16.55 & 0.00 & 8.20 & 0.17 & 6.32 & 11.37 \\
\hline R05-13r & 50.63 & 1.01 & 16.48 & 0.00 & 8.25 & 0.18 & 6.36 & 11.31 \\
\hline R05-13r & 50.66 & 0.98 & 16.48 & 0.01 & 8.24 & 0.16 & 6.26 & 11.33 \\
\hline R05-13r & 50.95 & 1.04 & 16.45 & 0.00 & 8.23 & 0.15 & 6.20 & 11.34 \\
\hline R05-13r & 50.88 & 1.00 & 16.30 & 0.04 & 8.28 & 0.19 & 6.38 & 11.19 \\
\hline R05-13r & 51.05 & 0.96 & 16.06 & 0.01 & 8.32 & 0.14 & 7.19 & 11.06 \\
\hline R05-13r & 51.01 & 1.00 & 16.47 & 0.00 & 8.16 & 0.15 & 6.48 & 11.36 \\
\hline
\end{tabular}




\begin{tabular}{|c|c|c|c|c|c|c|c|c|}
\hline $\mathrm{R} 05-13 \mathrm{r}$ & 50.51 & 0.99 & 16.56 & 0.01 & 8.19 & 0.16 & 6.41 & 11.32 \\
\hline $\mathrm{R} 05-13 r$ & 50.37 & 1.01 & 16.25 & 0.00 & 8.25 & 0.18 & 5.93 & 11.35 \\
\hline R05-13r & 51.01 & 0.99 & 16.43 & 0.00 & 7.45 & 0.12 & 6.30 & 11.32 \\
\hline R05-14r & 50.32 & 0.98 & 16.52 & 0.01 & 8.25 & 0.17 & 6.34 & 11.31 \\
\hline R05-14r & 50.69 & 1.02 & 16.51 & 0.02 & 8.26 & 0.15 & 6.27 & 11.38 \\
\hline$R 05-14 r$ & 51.01 & 0.95 & 16.28 & 0.00 & 8.12 & 0.16 & 6.24 & 10.97 \\
\hline R05-14r & 50.94 & 0.96 & 16.57 & 0.01 & 7.85 & 0.18 & 6.33 & 11.22 \\
\hline$R 05-14 r$ & 50.97 & 0.99 & 16.38 & 0.00 & 8.06 & 0.12 & 6.25 & 11.35 \\
\hline$R 05-14 r$ & 50.90 & 0.96 & 16.24 & 0.02 & 8.27 & 0.15 & 6.32 & 11.13 \\
\hline$R 05-14 r$ & 50.97 & 0.97 & 16.42 & 0.00 & 8.21 & 0.14 & 6.03 & 11.11 \\
\hline $\mathrm{R} 05-14 \mathrm{r}$ & 51.06 & 1.05 & 16.51 & 0.01 & 8.12 & 0.15 & 6.42 & 11.17 \\
\hline$R 05-14 r$ & 51.01 & 0.94 & 16.08 & 0.01 & 7.77 & 0.16 & 6.28 & 11.31 \\
\hline$R 05-14 r$ & 50.92 & 0.96 & 16.38 & 0.03 & 8.22 & 0.14 & 6.32 & 11.15 \\
\hline$R 05-14 r$ & 51.05 & 1.02 & 16.48 & 0.01 & 8.22 & 0.14 & 6.32 & 11.26 \\
\hline R05-14r & 50.74 & 0.93 & 15.53 & 0.02 & 8.50 & 0.15 & 7.72 & 10.91 \\
\hline$R 05-14 r$ & 51.05 & 0.98 & 16.53 & 0.00 & 8.26 & 0.15 & 6.27 & 11.35 \\
\hline$R 05-14 r$ & 50.98 & 1.00 & 16.55 & 0.00 & 8.28 & 0.16 & 6.27 & 11.28 \\
\hline$R 05-14 r$ & 51.03 & 0.99 & 16.30 & 0.01 & 7.71 & 0.16 & 6.18 & 11.21 \\
\hline $\mathrm{R} 05-17 \mathrm{r}$ & 50.24 & 0.99 & 16.44 & 0.00 & 8.14 & 0.19 & 6.45 & 11.33 \\
\hline $\mathrm{R} 05-17 r$ & 50.86 & 0.96 & 16.44 & 0.01 & 7.04 & 0.18 & 6.11 & 11.29 \\
\hline R05-17r & 50.83 & 1.00 & 16.32 & 0.00 & 8.16 & 0.18 & 6.34 & 10.99 \\
\hline $\mathrm{R} 05-17 \mathrm{r}$ & 51.03 & 0.95 & 16.49 & 0.00 & 7.95 & 0.16 & 6.32 & 11.34 \\
\hline $\mathrm{R} 05-17 \mathrm{r}$ & 51.07 & 0.98 & 16.50 & 0.01 & 8.13 & 0.17 & 6.35 & 11.36 \\
\hline $\mathrm{R} 05-17 r$ & 50.77 & 0.96 & 16.45 & 0.01 & 8.19 & 0.16 & 6.30 & 11.42 \\
\hline R05-17r & 51.02 & 1.03 & 16.49 & 0.00 & 7.83 & 0.19 & 6.08 & 11.41 \\
\hline $\mathrm{R} 05-17 \mathrm{r}$ & 50.69 & 0.93 & 15.98 & 0.02 & 8.03 & 0.16 & 7.76 & 11.17 \\
\hline $\mathrm{R} 05-17 r$ & 50.78 & 0.99 & 16.42 & 0.00 & 8.02 & 0.14 & 6.27 & 11.15 \\
\hline $\mathrm{R} 05-17 r$ & 50.27 & 0.94 & 16.31 & 0.00 & 7.85 & 0.13 & 6.18 & 10.89 \\
\hline $\mathrm{R} 05-17 \mathrm{r}$ & 51.12 & 0.97 & 16.52 & 0.03 & 8.08 & 0.18 & 6.29 & 11.40 \\
\hline $\mathrm{R} 05-17 \mathrm{r}$ & 51.21 & 0.94 & 16.44 & 0.01 & 8.24 & 0.16 & 6.18 & 11.55 \\
\hline $\mathrm{R} 05-17 \mathrm{r}$ & 50.90 & 0.96 & 16.55 & 0.02 & 8.19 & 0.13 & 6.23 & 11.37 \\
\hline $\mathrm{R} 05-17 r$ & 51.35 & 0.97 & 16.48 & 0.00 & 8.14 & 0.14 & 6.34 & 11.35 \\
\hline $\mathrm{R} 05-17 r$ & 51.10 & 0.97 & 16.54 & 0.03 & 8.17 & 0.16 & 6.24 & 11.43 \\
\hline R05-19r & 51.80 & 1.19 & 16.34 & 0.00 & 8.86 & 0.16 & 4.77 & 9.98 \\
\hline R05-19r & 51.86 & 1.09 & 16.30 & 0.00 & 8.63 & 0.16 & 5.20 & 10.00 \\
\hline R05-19r & 51.94 & 1.16 & 16.16 & 0.00 & 8.92 & 0.14 & 5.22 & 9.90 \\
\hline R05-19r & 51.67 & 1.16 & 16.37 & 0.01 & 8.71 & 0.15 & 5.27 & 9.76 \\
\hline R05-19r & 51.79 & 1.15 & 16.40 & 0.01 & 8.84 & 0.13 & 5.28 & 9.94 \\
\hline R05-19r & 51.76 & 1.12 & 16.20 & 0.02 & 8.92 & 0.18 & 4.96 & 9.88 \\
\hline R05-19r & 51.75 & 1.13 & 16.24 & 0.00 & 8.94 & 0.17 & 5.29 & 10.07 \\
\hline R05-19r & 51.57 & 1.14 & 16.08 & 0.00 & 8.94 & 0.13 & 5.16 & 10.01 \\
\hline R05-19r & 51.62 & 1.17 & 16.18 & 0.00 & 8.99 & 0.15 & 5.16 & 9.94 \\
\hline R05-19r & 52.10 & 1.12 & 16.21 & 0.00 & 8.84 & 0.16 & 5.24 & 9.63 \\
\hline R05-19r & 51.93 & 1.17 & 16.24 & 0.00 & 8.86 & 0.17 & 5.06 & 9.93 \\
\hline R05-19r & 51.93 & 1.18 & 16.00 & 0.01 & 8.75 & 0.14 & 5.20 & 9.92 \\
\hline R05-19r & 51.82 & 1.18 & 16.01 & 0.00 & 8.88 & 0.15 & 5.21 & 9.83 \\
\hline R05-19r & 51.73 & 1.18 & 16.04 & 0.00 & 8.63 & 0.16 & 5.03 & 10.05 \\
\hline
\end{tabular}




\begin{tabular}{|c|c|c|c|c|c|c|c|c|}
\hline R05-19r & 51.65 & 1.16 & 16.19 & 0.00 & 8.77 & 0.14 & 5.20 & 10.02 \\
\hline R05-10r & 50.64 & 0.99 & 16.68 & 0.01 & 7.89 & 0.16 & 6.48 & 11.35 \\
\hline R05-10r & 50.83 & 1.04 & 16.49 & 0.01 & 8.18 & 0.14 & 6.36 & 11.40 \\
\hline R05-10r & 50.54 & 1.00 & 16.35 & 0.01 & 8.25 & 0.16 & 6.01 & 11.28 \\
\hline R05-10r & 50.25 & 1.00 & 16.39 & 0.01 & 8.10 & 0.16 & 6.26 & 11.16 \\
\hline R05-10r & 50.90 & 0.99 & 16.17 & 0.00 & 8.05 & 0.12 & 6.40 & 10.74 \\
\hline R05-10r & 50.91 & 0.97 & 16.48 & 0.00 & 7.91 & 0.15 & 6.42 & 11.18 \\
\hline R05-10r & 50.92 & 1.03 & 16.42 & 0.03 & 8.03 & 0.16 & 6.46 & 11.30 \\
\hline R05-10r & 50.83 & 1.00 & 16.61 & 0.00 & 8.13 & 0.17 & 6.39 & 11.09 \\
\hline R05-10r & 50.84 & 0.95 & 16.47 & 0.02 & 8.23 & 0.16 & 6.16 & 11.27 \\
\hline R05-10r & 50.85 & 1.00 & 16.46 & 0.00 & 8.17 & 0.14 & 6.36 & 11.36 \\
\hline R05-10r & 50.66 & 1.01 & 16.49 & 0.00 & 8.11 & 0.13 & 6.40 & 11.27 \\
\hline R05-10r & 50.67 & 1.03 & 16.53 & 0.05 & 8.02 & 0.15 & 6.38 & 11.33 \\
\hline R05-10r & 50.28 & 0.99 & 16.38 & 0.01 & 8.07 & 0.16 & 6.20 & 11.42 \\
\hline R05-10r & 50.22 & 0.97 & 16.21 & 0.03 & 8.16 & 0.14 & 6.27 & 11.25 \\
\hline R05-10r & 50.40 & 1.01 & 16.24 & 0.02 & 7.92 & 0.17 & 6.31 & 11.39 \\
\hline R05-11 & 51.52 & 1.12 & 16.37 & 0.00 & 8.85 & 0.17 & 5.20 & 9.80 \\
\hline R05-11 & 50.92 & 1.17 & 16.36 & 0.01 & 8.58 & 0.15 & 5.26 & 9.88 \\
\hline R05-11 & 51.18 & 1.12 & 16.45 & 0.00 & 8.88 & 0.18 & 5.28 & 9.87 \\
\hline R05-11 & 51.66 & 1.10 & 16.26 & 0.00 & 8.00 & 0.18 & 5.28 & 9.82 \\
\hline R05-11 & 51.37 & 1.15 & 15.26 & 0.01 & 8.67 & 0.15 & 6.13 & 10.79 \\
\hline R05-11 & 51.45 & 1.12 & 16.52 & 0.00 & 8.42 & 0.19 & 4.90 & 9.87 \\
\hline R05-11 & 51.61 & 1.12 & 16.41 & 0.00 & 8.88 & 0.16 & 5.25 & 9.90 \\
\hline R05-11 & 51.63 & 1.12 & 16.29 & 0.00 & 8.88 & 0.18 & 5.23 & 9.80 \\
\hline R05-11 & 51.83 & 1.14 & 16.37 & 0.01 & 8.71 & 0.14 & 5.25 & 9.94 \\
\hline R05-11 & 51.36 & 1.17 & 16.27 & 0.01 & 8.61 & 0.16 & 5.32 & 9.83 \\
\hline R05-11 & 51.63 & 1.06 & 16.28 & 0.01 & 8.73 & 0.16 & 5.34 & 9.78 \\
\hline R05-11 & 51.17 & 1.18 & 16.28 & 0.01 & 8.91 & 0.14 & 5.13 & 9.26 \\
\hline R05-11 & 51.68 & 1.14 & 16.36 & 0.01 & 8.85 & 0.15 & 4.84 & 9.78 \\
\hline R05-11 & 51.65 & 1.01 & 14.02 & 0.04 & 8.25 & 0.17 & 7.40 & 12.18 \\
\hline R05-11 & 51.72 & 1.15 & 16.30 & 0.00 & 8.93 & 0.16 & 5.26 & 9.87 \\
\hline R05-12 & 50.72 & 0.94 & 16.51 & 0.02 & 8.06 & 0.20 & 6.46 & 11.00 \\
\hline R05-12 & 50.77 & 1.01 & 16.43 & 0.02 & 8.15 & 0.15 & 6.49 & 11.27 \\
\hline R05-12 & 50.86 & 0.98 & 15.91 & 0.02 & 8.13 & 0.14 & 6.24 & 11.37 \\
\hline R05-12 & 50.23 & 0.99 & 16.51 & 0.02 & 8.07 & 0.12 & 6.49 & 11.14 \\
\hline R05-12 & 51.09 & 0.95 & 16.53 & 0.03 & 8.21 & 0.14 & 6.40 & 11.27 \\
\hline R05-12 & 50.94 & 0.97 & 16.52 & 0.00 & 8.17 & 0.16 & 6.36 & 11.09 \\
\hline R05-12 & 50.84 & 0.95 & 16.49 & 0.02 & 8.11 & 0.14 & 6.50 & 11.18 \\
\hline R05-12 & 51.15 & 0.97 & 16.62 & 0.01 & 8.13 & 0.14 & 6.44 & 11.23 \\
\hline R05-12 & 50.99 & 0.93 & 16.42 & 0.01 & 8.11 & 0.15 & 6.47 & 11.22 \\
\hline R05-12 & 50.98 & 1.02 & 16.65 & 0.00 & 8.11 & 0.16 & 6.42 & 11.30 \\
\hline R05-13 & 50.93 & 0.99 & 16.42 & 0.02 & 8.16 & 0.15 & 6.49 & 11.35 \\
\hline R05-13 & 50.71 & 0.99 & 16.42 & 0.02 & 7.99 & 0.16 & 6.47 & 11.02 \\
\hline R05-13 & 51.22 & 0.95 & 16.53 & 0.00 & 8.07 & 0.14 & 6.46 & 11.23 \\
\hline R05-13 & 50.95 & 1.02 & 16.42 & 0.00 & 8.18 & 0.16 & 6.42 & 11.41 \\
\hline R05-13 & 50.89 & 0.98 & 16.48 & 0.00 & 8.15 & 0.19 & 6.47 & 11.18 \\
\hline R05-13 & 51.17 & 0.96 & 16.46 & 0.03 & 8.18 & 0.13 & 6.48 & 11.14 \\
\hline
\end{tabular}




\begin{tabular}{|c|c|c|c|c|c|c|c|c|}
\hline R05-13 & 51.16 & 0.97 & 16.45 & 0.02 & 8.11 & 0.14 & 6.49 & 11.23 \\
\hline R05-13 & 51.08 & 1.00 & 16.37 & 0.00 & 8.14 & 0.14 & 6.45 & 11.24 \\
\hline R05-13 & 50.56 & 1.02 & 16.29 & 0.00 & 8.09 & 0.13 & 6.37 & 11.28 \\
\hline R05-13 & 50.97 & 0.98 & 16.53 & 0.01 & 8.05 & 0.18 & 6.40 & 11.28 \\
\hline R05-14 & 50.79 & 0.95 & 16.31 & 0.00 & 7.91 & 0.17 & 6.51 & 11.22 \\
\hline R05-14 & 50.65 & 1.03 & 16.36 & 0.02 & 8.13 & 0.16 & 6.50 & 11.12 \\
\hline R05-14 & 50.87 & 0.95 & 16.48 & 0.01 & 8.19 & 0.16 & 6.43 & 11.30 \\
\hline R05-14 & 50.95 & 1.00 & 16.23 & 0.00 & 8.21 & 0.13 & 6.11 & 11.31 \\
\hline R05-14 & 50.62 & 0.98 & 16.37 & 0.03 & 8.09 & 0.15 & 6.41 & 11.30 \\
\hline R05-14 & 51.04 & 0.97 & 16.56 & 0.00 & 8.31 & 0.15 & 6.48 & 11.36 \\
\hline R05-14 & 50.50 & 0.99 & 16.49 & 0.01 & 8.08 & 0.15 & 6.37 & 11.26 \\
\hline R05-14 & 51.03 & 0.93 & 16.42 & 0.00 & 8.17 & 0.13 & 6.04 & 11.33 \\
\hline R05-14 & 50.95 & 1.05 & 16.34 & 0.01 & 8.05 & 0.13 & 6.40 & 10.60 \\
\hline R05-14 & 51.09 & 1.02 & 16.40 & 0.00 & 8.12 & 0.13 & 6.42 & 11.25 \\
\hline R05-16 & 50.99 & 0.98 & 16.45 & 0.02 & 8.23 & 0.13 & 6.53 & 11.29 \\
\hline R05-16 & 50.85 & 0.97 & 16.37 & 0.04 & 8.12 & 0.17 & 6.51 & 11.35 \\
\hline R05-16 & 50.94 & 0.96 & 16.56 & 0.00 & 8.06 & 0.14 & 6.46 & 11.33 \\
\hline R05-16 & 50.84 & 1.03 & 16.41 & 0.02 & 8.15 & 0.18 & 6.54 & 11.20 \\
\hline R05-16 & 50.95 & 1.01 & 16.48 & 0.03 & 7.89 & 0.19 & 6.50 & 11.10 \\
\hline R05-16 & 50.93 & 0.97 & 16.40 & 0.02 & 8.00 & 0.16 & 6.39 & 11.35 \\
\hline R05-16 & 50.84 & 0.97 & 16.51 & 0.00 & 8.19 & 0.14 & 6.44 & 11.38 \\
\hline R05-16 & 50.53 & 0.96 & 16.46 & 0.03 & 8.16 & 0.12 & 6.41 & 11.21 \\
\hline R05-16 & 50.97 & 0.96 & 16.01 & 0.01 & 8.03 & 0.14 & 6.57 & 11.02 \\
\hline R05-16 & 50.72 & 1.01 & 16.25 & 0.00 & 8.25 & 0.14 & 6.48 & 11.21 \\
\hline R06 & $\mathrm{SiO} 2$ & TiO2 & Al2O3 & $\mathrm{Cr} 2 \mathrm{O} 3$ & $\mathrm{FeO}$ & $\mathrm{MnO}$ & $\mathrm{MgO}$ & $\mathrm{CaO}$ \\
\hline R06-11r & 51.03 & 1.16 & 16.44 & 0.01 & 8.59 & 0.19 & 5.28 & 10.08 \\
\hline R06-11r & 51.55 & 1.13 & 16.46 & 0.02 & 8.73 & 0.17 & 5.07 & 10.01 \\
\hline R06-11r & 51.77 & 1.18 & 16.49 & 0.00 & 8.80 & 0.17 & 5.30 & 10.13 \\
\hline R06-11r & 51.51 & 1.17 & 16.41 & 0.00 & 8.64 & 0.19 & 5.24 & 9.84 \\
\hline R06-11r & 51.64 & 1.12 & 16.33 & 0.00 & 8.83 & 0.15 & 5.36 & 10.01 \\
\hline R06-11r & 51.55 & 1.14 & 16.32 & 0.00 & 8.54 & 0.18 & 5.17 & 10.19 \\
\hline R06-11r & 51.75 & 1.14 & 16.47 & 0.00 & 8.77 & 0.15 & 5.25 & 10.08 \\
\hline R06-11r & 51.66 & 1.13 & 16.50 & 0.00 & 8.76 & 0.15 & 5.27 & 10.10 \\
\hline R06-11r & 51.60 & 1.13 & 16.04 & 0.01 & 8.69 & 0.14 & 5.28 & 9.94 \\
\hline R06-11r & 51.67 & 1.07 & 16.51 & 0.01 & 8.78 & 0.15 & 5.32 & 10.00 \\
\hline R06-11r & 51.68 & 1.07 & 14.49 & 0.03 & 8.49 & 0.19 & 7.23 & 11.77 \\
\hline R06-11r & 51.78 & 1.12 & 16.30 & 0.00 & 8.68 & 0.15 & 5.25 & 10.01 \\
\hline R06-11r & 51.74 & 1.07 & 16.24 & 0.03 & 8.87 & 0.15 & 5.32 & 9.98 \\
\hline R06-11r & 51.82 & 1.14 & 16.49 & 0.02 & 8.91 & 0.13 & 5.33 & 9.73 \\
\hline R06-11r & 51.88 & 1.14 & 16.33 & 0.02 & 8.60 & 0.15 & 5.34 & 10.12 \\
\hline$R 06-12 r$ & 50.97 & 1.04 & 16.58 & 0.01 & 8.48 & 0.13 & 6.34 & 11.30 \\
\hline$R 06-12 r$ & 50.99 & 1.02 & 16.51 & 0.01 & 8.53 & 0.17 & 6.38 & 11.25 \\
\hline $\mathrm{R} 06-12 r$ & 51.18 & 1.01 & 16.62 & 0.00 & 8.48 & 0.15 & 6.32 & 11.00 \\
\hline $\mathrm{R} 06-12 r$ & 51.06 & 1.05 & 16.52 & 0.01 & 8.38 & 0.14 & 6.28 & 11.33 \\
\hline$R 06-12 r$ & 50.92 & 1.00 & 16.52 & 0.01 & 8.33 & 0.15 & 6.46 & 11.21 \\
\hline R06-12r & 50.66 & 1.06 & 16.47 & 0.00 & 8.29 & 0.18 & 6.27 & 11.37 \\
\hline
\end{tabular}




\begin{tabular}{|c|c|c|c|c|c|c|c|c|}
\hline R06-12r & 50.99 & 1.07 & 16.68 & 0.01 & 8.41 & 0.16 & 6.25 & 11.03 \\
\hline R06-12r & 51.07 & 1.02 & 16.53 & 0.00 & 8.25 & 0.16 & 6.36 & 11.37 \\
\hline R06-12r & 51.16 & 1.05 & 16.46 & 0.02 & 8.36 & 0.15 & 6.31 & 11.28 \\
\hline$R 06-12 r$ & 51.08 & 1.05 & 15.86 & 0.02 & 8.32 & 0.13 & 6.31 & 11.42 \\
\hline R06-12r & 51.12 & 0.98 & 16.54 & 0.01 & 8.52 & 0.17 & 5.93 & 11.33 \\
\hline R06-12r & 50.62 & 1.03 & 16.55 & 0.01 & 8.20 & 0.16 & 6.15 & 11.15 \\
\hline R06-12r & 50.90 & 1.04 & 16.59 & 0.01 & 8.28 & 0.11 & 6.23 & 11.29 \\
\hline$R 06-12 r$ & 50.39 & 1.07 & 16.55 & 0.00 & 8.26 & 0.17 & 6.22 & 11.31 \\
\hline R06-12r & 50.34 & 0.99 & 16.56 & 0.00 & 8.39 & 0.14 & 6.24 & 11.34 \\
\hline$R 06-13 r$ & 51.09 & 1.04 & 16.63 & 0.01 & 8.36 & 0.15 & 6.31 & 11.43 \\
\hline R06-13r & 51.05 & 0.99 & 16.16 & 0.01 & 8.47 & 0.15 & 6.38 & 11.34 \\
\hline$R 06-13 r$ & 51.07 & 1.00 & 16.60 & 0.00 & 8.46 & 0.16 & 6.36 & 11.38 \\
\hline R06-13r & 50.84 & 0.96 & 16.65 & 0.01 & 8.47 & 0.14 & 6.41 & 11.41 \\
\hline R06-13r & 50.83 & 1.01 & 16.25 & 0.02 & 8.14 & 0.16 & 6.35 & 11.36 \\
\hline$R 06-13 r$ & 51.04 & 1.05 & 16.56 & 0.00 & 8.38 & 0.17 & 6.29 & 11.36 \\
\hline R06-13r & 50.86 & 1.00 & 16.49 & 0.00 & 8.21 & 0.16 & 6.28 & 11.47 \\
\hline$R 06-13 r$ & 50.89 & 1.00 & 16.50 & 0.00 & 8.27 & 0.17 & 6.35 & 11.48 \\
\hline$R 06-13 r$ & 51.02 & 1.05 & 16.55 & 0.01 & 8.52 & 0.14 & 6.38 & 11.32 \\
\hline R06-13r & 50.56 & 0.99 & 16.16 & 0.00 & 8.47 & 0.15 & 6.24 & 11.40 \\
\hline$R 06-13 r$ & 50.87 & 1.04 & 16.34 & 0.01 & 8.20 & 0.13 & 6.35 & 11.40 \\
\hline$R 06-13 r$ & 50.95 & 1.04 & 16.49 & 0.01 & 8.46 & 0.14 & 6.01 & 11.44 \\
\hline$R 06-13 r$ & 50.91 & 1.02 & 16.22 & 0.00 & 8.39 & 0.17 & 6.37 & 11.27 \\
\hline R06-13r & 50.99 & 1.05 & 16.53 & 0.00 & 8.37 & 0.18 & 6.38 & 11.34 \\
\hline$R 06-13 r$ & 50.90 & 1.05 & 16.42 & 0.01 & 8.41 & 0.17 & 6.33 & 11.13 \\
\hline$R 06-14 r$ & 51.37 & 1.10 & 16.64 & 0.01 & 8.69 & 0.15 & 4.89 & 9.85 \\
\hline$R 06-14 r$ & 51.73 & 1.17 & 16.63 & 0.00 & 8.75 & 0.17 & 4.89 & 9.75 \\
\hline R06-14r & 51.64 & 1.12 & 16.18 & 0.01 & 8.83 & 0.15 & 4.95 & 9.51 \\
\hline R06-14r & 51.60 & 1.17 & 16.37 & 0.00 & 8.72 & 0.16 & 4.89 & 9.79 \\
\hline$R 06-14 r$ & 51.58 & 1.20 & 16.61 & 0.01 & 8.74 & 0.18 & 4.91 & 9.89 \\
\hline R06-14r & 49.93 & 1.10 & 15.85 & 0.00 & 8.53 & 0.19 & 5.12 & 10.00 \\
\hline R06-14r & 51.68 & 1.20 & 16.24 & 0.01 & 8.69 & 0.14 & 4.98 & 10.02 \\
\hline R06-14r & 51.75 & 1.16 & 16.33 & 0.00 & 8.59 & 0.19 & 5.10 & 10.24 \\
\hline$R 06-14 r$ & 51.64 & 1.10 & 16.65 & 0.00 & 8.66 & 0.15 & 4.90 & 9.92 \\
\hline R06-14r & 51.56 & 1.13 & 16.40 & 0.02 & 8.59 & 0.17 & 5.04 & 10.17 \\
\hline R06-14r & 51.68 & 1.10 & 16.68 & 0.00 & 8.78 & 0.15 & 4.85 & 10.04 \\
\hline R06-14r & 51.54 & 1.15 & 16.68 & 0.00 & 8.73 & 0.15 & 4.89 & 9.94 \\
\hline R06-14r & 51.70 & 1.20 & 16.25 & 0.03 & 8.52 & 0.16 & 4.91 & 9.82 \\
\hline R06-15r & 51.59 & 1.15 & 16.12 & 0.00 & 8.94 & 0.18 & 5.44 & 9.93 \\
\hline R06-15r & 51.77 & 1.14 & 16.26 & 0.00 & 9.11 & 0.16 & 5.34 & 9.94 \\
\hline R06-15r & 52.04 & 1.10 & 16.35 & 0.00 & 8.89 & 0.15 & 5.37 & 9.94 \\
\hline R06-15r & 51.81 & 1.14 & 16.46 & 0.00 & 8.82 & 0.14 & 5.29 & 9.93 \\
\hline$R 06-15 r$ & 51.66 & 1.10 & 16.35 & 0.01 & 8.95 & 0.16 & 5.27 & 10.02 \\
\hline$R 06-15 r$ & 51.84 & 1.16 & 16.22 & 0.00 & 8.92 & 0.15 & 5.40 & 10.02 \\
\hline R06-15r & 50.98 & 1.14 & 16.27 & 0.01 & 9.05 & 0.12 & 5.09 & 9.16 \\
\hline$R 06-15 r$ & 51.85 & 1.16 & 16.36 & 0.02 & 8.91 & 0.15 & 5.40 & 9.85 \\
\hline$R 06-15 r$ & 51.97 & 1.08 & 16.45 & 0.02 & 9.00 & 0.18 & 5.03 & 10.03 \\
\hline R06-15r & 51.71 & 1.15 & 16.51 & 0.00 & 8.81 & 0.18 & 5.26 & 10.03 \\
\hline
\end{tabular}




\begin{tabular}{|c|c|c|c|c|c|c|c|c|}
\hline R06-15r & 51.41 & 1.09 & 15.91 & 0.01 & 9.13 & 0.16 & 6.77 & 9.40 \\
\hline$R 06-15 r$ & 51.89 & 1.07 & 16.15 & 0.01 & 8.97 & 0.14 & 5.35 & 9.66 \\
\hline R06-15r & 51.73 & 1.15 & 16.44 & 0.01 & 8.55 & 0.16 & 5.37 & 9.98 \\
\hline $\mathrm{R} 06-15 \mathrm{r}$ & 51.87 & 1.18 & 16.31 & 0.01 & 8.82 & 0.17 & 5.31 & 10.05 \\
\hline R06-15r & 51.70 & 1.15 & 16.40 & 0.00 & 8.96 & 0.12 & 5.31 & 9.84 \\
\hline R06-16r & 51.81 & 1.11 & 16.36 & 0.02 & 8.80 & 0.16 & 5.34 & 10.05 \\
\hline R06-16r & 51.74 & 1.07 & 16.34 & 0.03 & 8.85 & 0.16 & 5.41 & 10.15 \\
\hline R06-16r & 51.85 & 1.13 & 16.38 & 0.02 & 8.70 & 0.14 & 5.18 & 10.09 \\
\hline R06-16r & 51.70 & 1.14 & 16.46 & 0.01 & 8.90 & 0.16 & 5.39 & 10.06 \\
\hline R06-16r & 50.65 & 1.16 & 16.22 & 0.00 & 9.01 & 0.19 & 5.49 & 10.12 \\
\hline R06-16r & 51.30 & 1.10 & 16.17 & 0.00 & 8.89 & 0.17 & 5.43 & 10.01 \\
\hline R06-16r & 51.66 & 1.12 & 16.41 & 0.00 & 8.88 & 0.19 & 5.16 & 10.03 \\
\hline R06-16r & 51.21 & 1.17 & 16.06 & 0.00 & 8.83 & 0.16 & 5.37 & 10.06 \\
\hline R06-16r & 51.78 & 1.10 & 16.38 & 0.01 & 8.87 & 0.16 & 5.47 & 9.66 \\
\hline R06-16r & 51.80 & 1.14 & 16.41 & 0.03 & 8.80 & 0.16 & 5.33 & 10.14 \\
\hline R06-16r & 51.65 & 1.08 & 16.43 & 0.00 & 8.90 & 0.15 & 5.49 & 10.04 \\
\hline R06-16r & 50.89 & 1.13 & 16.38 & 0.00 & 8.80 & 0.18 & 5.44 & 10.10 \\
\hline R06-16r & 51.53 & 1.09 & 16.21 & 0.00 & 8.83 & 0.15 & 5.33 & 10.06 \\
\hline R06-16r & 51.87 & 1.18 & 16.46 & 0.00 & 8.86 & 0.16 & 5.40 & 10.07 \\
\hline R06-11 & 51.52 & 1.09 & 16.50 & 0.02 & 8.79 & 0.16 & 5.33 & 9.67 \\
\hline R06-11 & 51.56 & 1.12 & 16.46 & 0.01 & 8.76 & 0.18 & 5.32 & 9.99 \\
\hline R06-11 & 51.78 & 1.12 & 16.52 & 0.01 & 8.80 & 0.14 & 5.30 & 9.99 \\
\hline R06-11 & 51.50 & 1.14 & 16.42 & 0.02 & 8.69 & 0.18 & 5.49 & 9.91 \\
\hline R06-11 & 51.63 & 1.14 & 16.48 & 0.01 & 8.58 & 0.16 & 5.40 & 10.00 \\
\hline R06-11 & 51.74 & 1.15 & 16.43 & 0.01 & 8.60 & 0.17 & 5.37 & 10.00 \\
\hline R06-11 & 50.81 & 1.13 & 16.30 & 0.00 & 8.76 & 0.14 & 5.33 & 10.06 \\
\hline R06-11 & 51.59 & 1.08 & 16.49 & 0.00 & 8.82 & 0.16 & 5.05 & 10.06 \\
\hline R06-11 & 51.45 & 1.16 & 16.46 & 0.00 & 8.74 & 0.17 & 5.42 & 10.01 \\
\hline R06-11 & 51.70 & 1.19 & 16.44 & 0.00 & 8.44 & 0.13 & 5.32 & 9.99 \\
\hline R06-12 & 51.24 & 1.09 & 16.49 & 0.01 & 8.66 & 0.18 & 5.21 & 9.45 \\
\hline R06-12 & 51.37 & 1.10 & 16.81 & 0.02 & 8.72 & 0.16 & 4.79 & 9.73 \\
\hline R06-12 & 51.53 & 1.12 & 16.85 & 0.00 & 8.67 & 0.16 & 4.66 & 9.56 \\
\hline R06-12 & 51.47 & 1.19 & 16.71 & 0.02 & 8.86 & 0.15 & 4.86 & 9.77 \\
\hline R06-12 & 51.32 & 1.12 & 16.07 & 0.01 & 8.65 & 0.18 & 5.33 & 10.22 \\
\hline R06-12 & 51.65 & 1.15 & 16.69 & 0.00 & 8.72 & 0.18 & 4.93 & 9.70 \\
\hline R06-12 & 51.44 & 1.15 & 16.33 & 0.02 & 8.77 & 0.14 & 5.06 & 10.04 \\
\hline R06-12 & 51.13 & 1.16 & 16.42 & 0.00 & 8.38 & 0.15 & 5.16 & 9.97 \\
\hline R06-12 & 51.53 & 1.15 & 16.48 & 0.01 & 8.45 & 0.14 & 4.98 & 9.95 \\
\hline R06-12 & 51.64 & 1.14 & 16.43 & 0.00 & 8.62 & 0.14 & 5.09 & 9.92 \\
\hline R06-13 & 50.78 & 0.97 & 16.55 & 0.02 & 8.35 & 0.14 & 6.45 & 11.28 \\
\hline R06-13 & 50.78 & 1.10 & 16.17 & 0.01 & 8.21 & 0.14 & 6.39 & 11.32 \\
\hline R06-13 & 50.98 & 1.04 & 16.49 & 0.03 & 8.39 & 0.16 & 6.43 & 11.20 \\
\hline R06-13 & 50.98 & 1.03 & 16.55 & 0.01 & 8.50 & 0.18 & 6.15 & 11.02 \\
\hline R06-13 & 51.03 & 1.04 & 16.25 & 0.01 & 8.29 & 0.14 & 6.15 & 11.34 \\
\hline R06-13 & 50.73 & 1.00 & 16.36 & 0.01 & 8.37 & 0.13 & 6.46 & 11.27 \\
\hline R06-13 & 50.48 & 0.99 & 16.48 & 0.01 & 8.53 & 0.18 & 6.45 & 11.40 \\
\hline R06-13 & 51.02 & 1.00 & 16.58 & 0.00 & 8.28 & 0.15 & 6.50 & 11.2 \\
\hline
\end{tabular}




$\begin{array}{rrrrrrrrr}\text { R06-13 } & 50.94 & 1.01 & 16.37 & 0.00 & 8.44 & 0.15 & 6.46 & 11.38 \\ \text { R06-14 } & 51.62 & 1.23 & 16.64 & 0.01 & 8.66 & 0.17 & 4.95 & 9.87 \\ \text { R06-14 } & 51.47 & 1.12 & 16.62 & 0.01 & 8.84 & 0.16 & 4.97 & 9.81 \\ \text { R06-14 } & 51.41 & 1.11 & 16.63 & 0.00 & 8.84 & 0.18 & 4.99 & 9.79 \\ \text { R06-14 } & 51.68 & 1.13 & 16.39 & 0.00 & 8.71 & 0.16 & 5.08 & 10.05 \\ \text { R06-14 } & 51.51 & 1.14 & 16.53 & 0.01 & 8.73 & 0.15 & 4.95 & 10.01 \\ \text { R06-14 } & 51.68 & 1.12 & 16.45 & 0.00 & 8.52 & 0.12 & 4.88 & 9.91 \\ \text { R06-14 } & 51.39 & 1.09 & 16.50 & 0.00 & 8.48 & 0.17 & 4.81 & 9.60 \\ \text { R06-14 } & 51.08 & 1.19 & 16.48 & 0.00 & 8.63 & 0.12 & 4.96 & 9.70 \\ \text { R06-14 } & 51.60 & 1.13 & 16.62 & 0.00 & 8.82 & 0.15 & 5.00 & 9.74 \\ \text { R06-14 } & 51.64 & 1.16 & 16.47 & 0.00 & 8.76 & 0.18 & 4.90 & 9.89 \\ \text { R06-15 } & 51.94 & 1.23 & 16.27 & 0.02 & 8.90 & 0.15 & 5.48 & 9.98 \\ \text { R06-15 } & 51.56 & 1.15 & 16.16 & 0.00 & 8.81 & 0.18 & 5.45 & 9.80 \\ \text { R06-15 } & 51.83 & 1.22 & 16.18 & 0.02 & 8.94 & 0.18 & 5.49 & 9.99 \\ \text { R06-15 } & 51.78 & 1.16 & 16.25 & 0.01 & 8.75 & 0.13 & 5.37 & 10.00 \\ \text { R06-15 } & 51.79 & 1.13 & 16.27 & 0.02 & 8.68 & 0.16 & 5.32 & 9.91 \\ \text { R06-15 } & 51.74 & 1.16 & 15.86 & 0.00 & 8.84 & 0.16 & 5.35 & 9.90 \\ \text { R06-15 } & 51.99 & 1.13 & 16.33 & 0.00 & 8.88 & 0.12 & 5.42 & 9.50 \\ \text { R06-15 } & 51.67 & 1.13 & 16.02 & 0.01 & 8.84 & 0.18 & 5.35 & 10.01 \\ \text { R06-15 } & 51.65 & 1.15 & 15.99 & 0.00 & 8.93 & 0.21 & 5.19 & 9.98 \\ \text { R06-15 } & 50.75 & 1.03 & 15.11 & 0.00 & 9.77 & 0.15 & 8.85 & 8.96\end{array}$

\begin{tabular}{|c|c|c|c|c|c|c|c|c|}
\hline R15 & $\mathrm{SiO} 2$ & TiO2 & $\mathrm{Al} 2 \mathrm{O} 3$ & Cr2O3 & $\mathrm{FeO}$ & $\mathrm{MnO}$ & $\mathrm{MgO}$ & $\mathrm{CaO}$ \\
\hline R15-11 & 52.11 & 1.13 & 16.48 & 0.00 & 8.80 & 0.17 & 5.40 & 9.78 \\
\hline R15-11 & 52.02 & 1.13 & 16.41 & 0.00 & 8.85 & 0.18 & 5.40 & 10.02 \\
\hline R15-11 & 51.88 & 1.11 & 16.51 & 0.00 & 8.88 & 0.17 & 5.28 & 10.18 \\
\hline R15-11 & 52.04 & 1.13 & 16.39 & 0.00 & 8.72 & 0.15 & 5.38 & 10.07 \\
\hline R15-11 & 52.10 & 1.13 & 16.44 & 0.01 & 8.89 & 0.15 & 5.32 & 9.91 \\
\hline R15-11 & 52.11 & 1.16 & 16.55 & 0.00 & 8.97 & 0.17 & 5.34 & 9.83 \\
\hline R15-11 & 52.04 & 1.12 & 16.29 & 0.00 & 8.79 & 0.17 & 5.48 & 9.80 \\
\hline R15-11 & 51.96 & 1.15 & 16.50 & 0.00 & 8.67 & 0.19 & 5.45 & 10.00 \\
\hline R15-11 & 51.71 & 1.14 & 16.45 & 0.00 & 8.87 & 0.16 & 5.48 & 10.13 \\
\hline R15-11 & 52.05 & 1.15 & 16.43 & 0.00 & 8.89 & 0.15 & 5.44 & 10.11 \\
\hline R15-11 & 51.92 & 1.15 & 16.16 & 0.00 & 8.81 & 0.18 & 5.36 & 10.16 \\
\hline R15-11 & 51.99 & 1.17 & 16.34 & 0.02 & 8.88 & 0.16 & 5.41 & 10.07 \\
\hline R15-11 & 51.99 & 1.15 & 16.45 & 0.00 & 8.79 & 0.19 & 5.36 & 10.09 \\
\hline R15-11 & 52.00 & 1.14 & 16.39 & 0.00 & 8.86 & 0.15 & 5.42 & 10.13 \\
\hline R15-11 & 52.09 & 1.18 & 16.48 & 0.00 & 8.99 & 0.16 & 5.35 & 10.03 \\
\hline $\mathrm{R} 15-12 r$ & 52.02 & 1.19 & 16.42 & 0.00 & 8.97 & 0.15 & 5.49 & 10.10 \\
\hline $\mathrm{R} 15-12 r$ & 52.09 & 1.07 & 16.12 & 0.00 & 8.98 & 0.15 & 5.43 & 10.00 \\
\hline$R 15-12 r$ & 52.24 & 1.11 & 16.44 & 0.00 & 9.00 & 0.17 & 5.33 & 10.20 \\
\hline R15-12r & 52.02 & 1.14 & 16.38 & 0.00 & 8.72 & 0.18 & 5.51 & 10.00 \\
\hline$R 15-12 r$ & 51.59 & 1.19 & 16.29 & 0.01 & 8.87 & 0.16 & 5.60 & 10.32 \\
\hline$R 15-12 r$ & 51.94 & 1.14 & 16.60 & 0.03 & 8.78 & 0.19 & 5.39 & 10.04 \\
\hline$R 15-12 r$ & 51.01 & 1.14 & 16.33 & 0.01 & 9.00 & 0.16 & 5.43 & 10.08 \\
\hline R15-12r & 51.97 & 1.14 & 16.49 & 0.01 & 8.89 & 0.15 & 5.40 & 10.10 \\
\hline $\mathrm{R} 15-12 r$ & 52.12 & 1.12 & 16.48 & 0.03 & 9.00 & 0.14 & 5.46 & 9.8 \\
\hline
\end{tabular}




\begin{tabular}{|c|c|c|c|c|c|c|c|c|}
\hline R15-12r & 52.21 & 1.13 & 16.38 & 0.00 & 9.03 & 0.19 & 5.40 & 9.94 \\
\hline R15-12r & 52.21 & 1.12 & 16.50 & 0.00 & 8.96 & 0.16 & 5.42 & 10.13 \\
\hline R15-12r & 51.39 & 1.14 & 16.42 & 0.01 & 9.01 & 0.17 & 5.44 & 9.97 \\
\hline R15-12r & 51.87 & 1.15 & 16.50 & 0.00 & 8.91 & 0.14 & 5.32 & 10.07 \\
\hline$R 15-13 r$ & 52.08 & 1.16 & 16.61 & 0.01 & 8.90 & 0.15 & 5.47 & 9.49 \\
\hline R15-13r & 51.91 & 1.15 & 16.45 & 0.00 & 9.05 & 0.17 & 5.52 & 10.05 \\
\hline R15-13r & 51.90 & 1.14 & 16.50 & 0.00 & 8.75 & 0.17 & 5.09 & 9.96 \\
\hline$R 15-13 r$ & 52.18 & 1.17 & 16.20 & 0.00 & 8.92 & 0.16 & 5.35 & 10.01 \\
\hline R15-13r & 52.20 & 1.15 & 16.55 & 0.01 & 8.99 & 0.18 & 5.43 & 10.16 \\
\hline R15-13r & 51.92 & 1.14 & 16.50 & 0.00 & 8.91 & 0.18 & 5.34 & 10.19 \\
\hline R15-13r & 51.90 & 1.15 & 16.50 & 0.00 & 9.00 & 0.19 & 5.29 & 9.99 \\
\hline R15-13r & 51.96 & 1.15 & 16.42 & 0.02 & 9.05 & 0.15 & 5.50 & 9.84 \\
\hline R15-13r & 51.99 & 1.10 & 16.41 & 0.01 & 9.03 & 0.17 & 5.48 & 10.18 \\
\hline R15-13r & 52.12 & 1.15 & 16.49 & 0.00 & 9.03 & 0.16 & 5.44 & 9.96 \\
\hline$R 15-13 r$ & 52.06 & 1.21 & 16.52 & 0.00 & 8.89 & 0.16 & 5.31 & 10.16 \\
\hline$R 15-13 r$ & 52.09 & 1.17 & 16.50 & 0.01 & 8.85 & 0.18 & 5.44 & 10.06 \\
\hline R15-13r & 52.13 & 1.14 & 16.49 & 0.01 & 8.95 & 0.19 & 5.43 & 10.15 \\
\hline R15-13r & 52.19 & 1.15 & 16.53 & 0.00 & 9.01 & 0.15 & 5.42 & 9.96 \\
\hline R15-13r & 51.57 & 1.16 & 16.47 & 0.00 & 8.77 & 0.18 & 5.40 & 10.03 \\
\hline$R 15-14 r$ & 51.43 & 1.19 & 15.92 & 0.01 & 8.80 & 0.17 & 5.28 & 9.65 \\
\hline R15-14r & 52.13 & 1.23 & 16.38 & 0.00 & 8.95 & 0.15 & 5.11 & 10.01 \\
\hline R15-14r & 52.04 & 1.13 & 16.45 & 0.02 & 8.85 & 0.17 & 5.17 & 10.06 \\
\hline$R 15-14 r$ & 51.51 & 0.87 & 20.90 & 0.00 & 6.80 & 0.13 & 3.78 & 10.66 \\
\hline R15-14r & 52.03 & 1.14 & 16.42 & 0.00 & 8.81 & 0.15 & 5.23 & 9.96 \\
\hline R15-14r & 52.10 & 1.17 & 16.36 & 0.02 & 8.77 & 0.19 & 5.16 & 10.14 \\
\hline$R 15-14 r$ & 52.15 & 1.15 & 16.52 & 0.00 & 8.91 & 0.14 & 5.17 & 10.05 \\
\hline R15-14r & 51.97 & 1.14 & 16.27 & 0.02 & 8.87 & 0.19 & 5.28 & 9.99 \\
\hline R15-14r & 51.84 & 1.17 & 16.14 & 0.01 & 8.91 & 0.15 & 5.22 & 10.11 \\
\hline R15-14r & 51.99 & 1.10 & 16.48 & 0.00 & 8.49 & 0.16 & 5.21 & 10.15 \\
\hline R15-14r & 51.88 & 1.14 & 16.13 & 0.01 & 8.78 & 0.17 & 5.53 & 10.38 \\
\hline R15-14r & 51.84 & 1.15 & 16.09 & 0.01 & 8.83 & 0.14 & 5.26 & 9.87 \\
\hline R15-14r & 51.83 & 1.18 & 16.27 & 0.00 & 8.91 & 0.15 & 5.23 & 10.01 \\
\hline R15-15r & 52.14 & 1.12 & 16.28 & 0.00 & 8.83 & 0.17 & 5.32 & 9.96 \\
\hline R15-15r & 52.01 & 1.17 & 16.27 & 0.01 & 8.80 & 0.18 & 5.40 & 10.10 \\
\hline R15-15r & 51.94 & 1.20 & 16.47 & 0.00 & 8.92 & 0.16 & 5.45 & 10.04 \\
\hline R15-15r & 52.04 & 1.17 & 16.39 & 0.01 & 8.94 & 0.16 & 5.24 & 9.98 \\
\hline R15-15r & 50.85 & 1.18 & 16.53 & 0.00 & 8.84 & 0.16 & 5.34 & 10.06 \\
\hline R15-15r & 52.08 & 1.15 & 16.47 & 0.01 & 8.84 & 0.16 & 5.38 & 9.91 \\
\hline R15-15r & 51.73 & 1.14 & 15.99 & 0.00 & 8.96 & 0.17 & 5.32 & 10.02 \\
\hline R15-15r & 52.21 & 1.10 & 16.53 & 0.02 & 8.97 & 0.17 & 5.43 & 10.06 \\
\hline R15-15r & 51.87 & 1.17 & 16.39 & 0.01 & 8.90 & 0.16 & 5.38 & 9.90 \\
\hline R15-15r & 51.94 & 1.23 & 16.26 & 0.02 & 8.74 & 0.16 & 5.40 & 10.09 \\
\hline R15-15r & 51.90 & 1.14 & 16.46 & 0.00 & 9.07 & 0.16 & 5.36 & 10.01 \\
\hline R15-15r & 51.90 & 1.12 & 16.42 & 0.00 & 8.98 & 0.14 & 5.12 & 10.00 \\
\hline R15-15r & 52.01 & 1.16 & 16.52 & 0.01 & 8.92 & 0.15 & 5.37 & 9.71 \\
\hline R15-15r & 52.03 & 1.12 & 16.52 & 0.02 & 9.03 & 0.17 & 5.36 & 10.00 \\
\hline R15-15r & 51.97 & 1.17 & 16.55 & 0.00 & 8.88 & 0.16 & 5.39 & 10.08 \\
\hline
\end{tabular}




\begin{tabular}{|c|c|c|c|c|c|c|c|c|}
\hline R15-16r & 52.17 & 1.13 & 16.53 & 0.00 & 9.08 & 0.17 & 5.32 & 10.12 \\
\hline R15-16r & 52.03 & 1.20 & 16.64 & 0.00 & 8.77 & 0.15 & 5.22 & 9.93 \\
\hline R15-16r & 51.98 & 1.11 & 15.90 & 0.02 & 8.86 & 0.17 & 5.86 & 10.58 \\
\hline R15-16r & 52.20 & 1.09 & 16.41 & 0.00 & 9.00 & 0.15 & 5.25 & 9.96 \\
\hline R15-16r & 52.03 & 1.14 & 16.32 & 0.00 & 8.95 & 0.18 & 5.35 & 9.98 \\
\hline R15-16r & 52.19 & 1.13 & 16.33 & 0.01 & 8.71 & 0.17 & 5.33 & 9.98 \\
\hline R15-16r & 52.11 & 1.13 & 16.52 & 0.01 & 8.95 & 0.15 & 5.25 & 9.87 \\
\hline R15-16r & 52.24 & 1.15 & 16.43 & 0.01 & 8.64 & 0.18 & 5.37 & 10.04 \\
\hline$R 15-16 r$ & 51.89 & 1.14 & 16.33 & 0.00 & 8.94 & 0.19 & 5.02 & 9.77 \\
\hline R15-16r & 51.83 & 1.10 & 16.45 & 0.00 & 8.98 & 0.20 & 5.35 & 9.85 \\
\hline R15-16r & 51.92 & 1.25 & 16.44 & 0.00 & 8.90 & 0.19 & 5.23 & 10.02 \\
\hline R15-16r & 52.16 & 1.21 & 16.44 & 0.02 & 9.03 & 0.19 & 5.42 & 10.04 \\
\hline$R 15-16 r$ & 51.90 & 1.15 & 16.46 & 0.00 & 8.59 & 0.16 & 5.13 & 9.96 \\
\hline R15-16r & 51.26 & 1.16 & 15.47 & 0.01 & 9.00 & 0.16 & 6.16 & 11.47 \\
\hline R15-16r & 51.94 & 1.14 & 16.42 & 0.00 & 8.85 & 0.19 & 5.15 & 10.14 \\
\hline R15-12 & 52.20 & 1.16 & 15.95 & 0.00 & 9.01 & 0.16 & 5.46 & 10.04 \\
\hline R15-12 & 51.98 & 1.13 & 16.23 & 0.01 & 8.92 & 0.16 & 5.45 & 9.95 \\
\hline R15-12 & 51.57 & 1.16 & 16.20 & 0.00 & 9.00 & 0.16 & 6.04 & 9.62 \\
\hline R15-12 & 52.01 & 1.11 & 16.41 & 0.02 & 8.72 & 0.18 & 5.27 & 10.01 \\
\hline R15-12 & 51.50 & 0.90 & 20.40 & 0.02 & 6.58 & 0.14 & 4.16 & 11.01 \\
\hline R15-12 & 52.16 & 1.16 & 16.32 & 0.00 & 8.94 & 0.15 & 5.36 & 9.94 \\
\hline R15-12 & 52.06 & 1.17 & 16.34 & 0.00 & 8.84 & 0.19 & 5.39 & 9.97 \\
\hline R15-12 & 51.92 & 1.15 & 16.40 & 0.01 & 8.86 & 0.17 & 5.45 & 10.03 \\
\hline R15-12 & 52.31 & 1.15 & 16.35 & 0.00 & 8.99 & 0.16 & 5.42 & 9.99 \\
\hline R15-12 & 52.33 & 1.12 & 16.42 & 0.00 & 9.09 & 0.16 & 5.51 & 9.96 \\
\hline R15-14 & 51.91 & 1.10 & 17.53 & 0.00 & 8.29 & 0.16 & 4.87 & 10.44 \\
\hline R15-14 & 51.98 & 0.99 & 18.06 & 0.02 & 7.77 & 0.16 & 5.01 & 10.75 \\
\hline R15-14 & 51.67 & 1.10 & 16.14 & 0.01 & 8.96 & 0.16 & 5.31 & 10.05 \\
\hline R15-14 & 51.86 & 1.14 & 16.34 & 0.02 & 8.51 & 0.16 & 5.20 & 9.93 \\
\hline R15-14 & 52.03 & 1.15 & 16.22 & 0.00 & 8.88 & 0.17 & 5.45 & 9.89 \\
\hline R15-14 & 51.89 & 1.16 & 15.79 & 0.00 & 8.98 & 0.15 & 5.33 & 10.01 \\
\hline R15-14 & 51.90 & 1.22 & 16.46 & 0.01 & 8.88 & 0.15 & 5.09 & 10.01 \\
\hline R15-14 & 51.75 & 1.21 & 16.41 & 0.01 & 8.83 & 0.16 & 5.26 & 10.05 \\
\hline R15-14 & 51.60 & 1.17 & 15.50 & 0.00 & 8.90 & 0.17 & 5.37 & 9.36 \\
\hline R15-14 & 51.87 & 1.14 & 16.26 & 0.01 & 8.98 & 0.17 & 5.43 & 9.95 \\
\hline R15-15 & 52.04 & 1.14 & 16.52 & 0.01 & 9.02 & 0.16 & 5.46 & 9.82 \\
\hline R15-15 & 51.81 & 1.21 & 16.36 & 0.01 & 9.04 & 0.17 & 5.43 & 9.96 \\
\hline R15-15 & 52.11 & 1.21 & 16.31 & 0.00 & 8.96 & 0.11 & 5.52 & 10.00 \\
\hline R15-15 & 52.06 & 1.14 & 15.92 & 0.00 & 8.98 & 0.14 & 5.43 & 9.99 \\
\hline R15-15 & 51.62 & 1.11 & 16.26 & 0.00 & 8.92 & 0.19 & 5.34 & 9.80 \\
\hline R15-15 & 52.13 & 1.21 & 16.38 & 0.00 & 9.00 & 0.16 & 5.29 & 9.93 \\
\hline R15-15 & 52.01 & 1.13 & 16.30 & 0.00 & 8.96 & 0.18 & 5.26 & 9.91 \\
\hline R15-15 & 52.08 & 1.15 & 16.33 & 0.02 & 8.95 & 0.15 & 5.27 & 9.94 \\
\hline R15-15 & 51.54 & 1.14 & 15.67 & 0.00 & 9.59 & 0.16 & 7.43 & 9.25 \\
\hline R15-15 & 52.02 & 1.14 & 16.49 & 0.00 & 8.88 & 0.18 & 5.36 & 9.96 \\
\hline R15-16 & 52.03 & 1.18 & 16.70 & 0.00 & 8.93 & 0.16 & 4.92 & 9.74 \\
\hline R15-16 & 51.46 & 1.10 & 15.67 & 0.00 & 9.26 & 0.15 & 6.68 & 9.42 \\
\hline
\end{tabular}




\begin{tabular}{r|rrrrrrrr} 
R15-16 & 51.92 & 1.13 & 17.18 & 0.00 & 8.67 & 0.14 & 4.96 & 9.89 \\
R15-16 & 51.23 & 1.11 & 15.80 & 0.00 & 9.12 & 0.14 & 7.08 & 9.30 \\
R15-16 & 52.07 & 1.17 & 16.52 & 0.00 & 8.99 & 0.20 & 5.14 & 9.72 \\
R15-16 & 52.17 & 1.01 & 17.92 & 0.01 & 8.09 & 0.17 & 4.71 & 10.20 \\
R15-16 & 52.21 & 1.18 & 16.55 & 0.01 & 8.98 & 0.16 & 4.87 & 9.94 \\
R15-16 & 51.91 & 1.19 & 16.51 & 0.01 & 8.89 & 0.20 & 5.04 & 9.86
\end{tabular}




\begin{tabular}{|c|c|c|c|c|c|c|}
\hline $\mathrm{Na} 2 \mathrm{O}$ & $\mathrm{K} 2 \mathrm{O}$ & P2O5 & $\mathrm{F}$ & $\mathrm{Cl}$ & $\mathrm{SO} 3$ & Total \\
\hline 3.00 & 0.22 & 0.08 & 0.00 & 0.10 & 0.19 & 98.36 \\
\hline 2.77 & 0.20 & 0.13 & 0.00 & 0.09 & 0.19 & 98.02 \\
\hline 2.82 & 0.22 & 0.13 & 0.00 & 0.10 & 0.20 & 97.38 \\
\hline 2.86 & 0.20 & 0.13 & 0.00 & 0.08 & 0.18 & 96.66 \\
\hline 2.83 & 0.22 & 0.14 & 0.08 & 0.09 & 0.18 & 97.87 \\
\hline 2.74 & 0.21 & 0.10 & 0.00 & 0.10 & 0.17 & 97.52 \\
\hline 2.76 & 0.22 & 0.13 & 0.09 & 0.09 & 0.15 & 97.58 \\
\hline 2.85 & 0.21 & 0.09 & 0.02 & 0.08 & 0.21 & 98.03 \\
\hline 2.83 & 0.21 & 0.12 & 0.00 & 0.08 & 0.19 & 97.50 \\
\hline 2.87 & 0.21 & 0.12 & 0.00 & 0.08 & 0.17 & 97.59 \\
\hline 2.81 & 0.22 & 0.07 & 0.00 & 0.10 & 0.20 & 97.30 \\
\hline 2.77 & 0.21 & 0.15 & 0.00 & 0.09 & 0.19 & 97.14 \\
\hline 2.88 & 0.23 & 0.11 & 0.07 & 0.09 & 0.17 & 98.00 \\
\hline 2.39 & 0.20 & 0.10 & 0.00 & 0.08 & 0.17 & 97.82 \\
\hline 2.53 & 0.22 & 0.10 & 0.00 & 0.11 & 0.17 & 97.58 \\
\hline 2.75 & 0.22 & 0.11 & 0.00 & 0.10 & 0.21 & 97.45 \\
\hline 2.87 & 0.23 & 0.14 & 0.00 & 0.09 & 0.21 & 97.88 \\
\hline 2.87 & 0.23 & 0.14 & 0.06 & 0.09 & 0.18 & 97.83 \\
\hline 2.78 & 0.22 & 0.14 & 0.00 & 0.10 & 0.21 & 97.45 \\
\hline 2.77 & 0.20 & 0.13 & 0.00 & 0.07 & 0.19 & 97.73 \\
\hline 2.74 & 0.21 & 0.10 & 0.00 & 0.10 & 0.17 & 97.47 \\
\hline 2.89 & 0.22 & 0.06 & 0.00 & 0.10 & 0.16 & 97.05 \\
\hline 2.86 & 0.21 & 0.14 & 0.00 & 0.10 & 0.21 & 98.05 \\
\hline 2.78 & 0.21 & 0.12 & 0.00 & 0.09 & 0.22 & 97.37 \\
\hline 2.84 & 0.22 & 0.11 & 0.19 & 0.09 & 0.19 & 98.24 \\
\hline 2.89 & 0.21 & 0.12 & 0.00 & 0.10 & 0.20 & 97.72 \\
\hline 2.28 & 0.21 & 0.13 & 0.00 & 0.10 & 0.20 & 97.07 \\
\hline 2.83 & 0.21 & 0.13 & 0.00 & 0.09 & 0.24 & 97.74 \\
\hline 2.81 & 0.24 & 0.10 & 0.05 & 0.11 & 0.18 & 98.02 \\
\hline 2.82 & 0.22 & 0.12 & 0.00 & 0.10 & 0.20 & 98.19 \\
\hline 2.84 & 0.20 & 0.08 & 0.00 & 0.08 & 0.19 & 97.54 \\
\hline 2.76 & 0.21 & 0.10 & 0.00 & 0.10 & 0.19 & 98.13 \\
\hline 2.89 & 0.23 & 0.14 & 0.08 & 0.10 & 0.18 & 97.62 \\
\hline 2.77 & 0.23 & 0.10 & 0.00 & 0.10 & 0.21 & 97.71 \\
\hline 2.83 & 0.21 & 0.09 & 0.00 & 0.09 & 0.21 & 97.50 \\
\hline 2.45 & 0.21 & 0.08 & 0.06 & 0.08 & 0.19 & 97.04 \\
\hline 2.75 & 0.21 & 0.11 & 0.09 & 0.09 & 0.18 & 96.96 \\
\hline 2.71 & 0.21 & 0.07 & 0.04 & 0.08 & 0.22 & 97.97 \\
\hline 2.86 & 0.22 & 0.10 & 0.05 & 0.09 & 0.18 & 97.36 \\
\hline 2.76 & 0.22 & 0.13 & 0.00 & 0.10 & 0.21 & 97.62 \\
\hline 2.86 & 0.22 & 0.17 & 0.00 & 0.09 & 0.19 & 97.65 \\
\hline 2.72 & 0.22 & 0.11 & 0.00 & 0.09 & 0.20 & 97.68 \\
\hline 2.75 & 0.23 & 0.11 & 0.00 & 0.08 & 0.20 & 97.61 \\
\hline 2.54 & 0.20 & 0.11 & 0.17 & 0.08 & 0.19 & 98.02 \\
\hline 2.81 & 0.20 & 0.14 & 0.02 & 0.08 & 0.20 & 98.05 \\
\hline
\end{tabular}




\begin{tabular}{|c|c|c|c|c|c|c|}
\hline 2.79 & 0.22 & 0.08 & 0.00 & 0.08 & 0.18 & 97.48 \\
\hline 2.33 & 0.22 & 0.07 & 0.00 & 0.08 & 0.21 & 96.24 \\
\hline 2.89 & 0.23 & 0.09 & 0.00 & 0.08 & 0.20 & 97.10 \\
\hline 2.77 & 0.23 & 0.16 & 0.00 & 0.10 & 0.18 & 97.33 \\
\hline 2.82 & 0.22 & 0.13 & 0.01 & 0.09 & 0.18 & 97.70 \\
\hline 2.86 & 0.23 & 0.12 & 0.00 & 0.09 & 0.19 & 97.20 \\
\hline 2.89 & 0.21 & 0.12 & 0.00 & 0.09 & 0.18 & 97.54 \\
\hline 2.82 & 0.22 & 0.11 & 0.00 & 0.09 & 0.17 & 97.51 \\
\hline 2.82 & 0.23 & 0.08 & 0.05 & 0.09 & 0.19 & 97.41 \\
\hline 2.84 & 0.22 & 0.14 & 0.00 & 0.07 & 0.20 & 97.31 \\
\hline 2.69 & 0.22 & 0.13 & 0.00 & 0.10 & 0.19 & 97.80 \\
\hline 2.83 & 0.23 & 0.12 & 0.25 & 0.09 & 0.20 & 97.14 \\
\hline 2.88 & 0.23 & 0.12 & 0.00 & 0.10 & 0.18 & 97.61 \\
\hline 2.89 & 0.21 & 0.12 & 0.00 & 0.09 & 0.20 & 97.99 \\
\hline 2.74 & 0.22 & 0.13 & 0.01 & 0.09 & 0.16 & 97.83 \\
\hline 2.57 & 0.21 & 0.11 & 0.00 & 0.09 & 0.21 & 97.75 \\
\hline 2.88 & 0.22 & 0.12 & 0.00 & 0.09 & 0.22 & 98.01 \\
\hline 2.85 & 0.20 & 0.09 & 0.06 & 0.10 & 0.20 & 97.04 \\
\hline 2.81 & 0.21 & 0.08 & 0.08 & 0.09 & 0.18 & 97.19 \\
\hline 2.80 & 0.21 & 0.12 & 0.02 & 0.08 & 0.18 & 96.28 \\
\hline 2.46 & 0.23 & 0.07 & 0.08 & 0.08 & 0.16 & 96.85 \\
\hline 2.65 & 0.21 & 0.11 & 0.18 & 0.09 & 0.18 & 97.56 \\
\hline 2.74 & 0.20 & 0.12 & 0.00 & 0.08 & 0.19 & 97.89 \\
\hline 2.79 & 0.21 & 0.14 & 0.00 & 0.10 & 0.17 & 97.65 \\
\hline 2.95 & 0.23 & 0.11 & 0.00 & 0.08 & 0.19 & 97.60 \\
\hline 2.69 & 0.21 & 0.12 & 0.01 & 0.09 & 0.19 & 98.04 \\
\hline 2.83 & 0.22 & 0.07 & 0.00 & 0.10 & 0.18 & 97.15 \\
\hline 2.78 & 0.21 & 0.11 & 0.00 & 0.09 & 0.16 & 95.90 \\
\hline 2.83 & 0.22 & 0.08 & 0.00 & 0.09 & 0.22 & 98.02 \\
\hline 2.75 & 0.22 & 0.10 & 0.00 & 0.11 & 0.22 & 98.09 \\
\hline 2.82 & 0.22 & 0.06 & 0.07 & 0.07 & 0.23 & 97.77 \\
\hline 2.75 & 0.21 & 0.11 & 0.00 & 0.09 & 0.18 & 98.09 \\
\hline 2.85 & 0.21 & 0.08 & 0.00 & 0.09 & 0.19 & 98.03 \\
\hline 3.13 & 0.26 & 0.11 & 0.09 & 0.13 & 0.17 & 96.92 \\
\hline 3.11 & 0.25 & 0.09 & 0.00 & 0.11 & 0.20 & 96.98 \\
\hline 3.19 & 0.26 & 0.11 & 0.00 & 0.15 & 0.24 & 97.35 \\
\hline 3.17 & 0.24 & 0.08 & 0.09 & 0.13 & 0.17 & 96.92 \\
\hline 3.16 & 0.26 & 0.13 & 0.07 & 0.14 & 0.21 & 97.43 \\
\hline 3.00 & 0.24 & 0.13 & 0.03 & 0.12 & 0.23 & 96.75 \\
\hline 3.16 & 0.25 & 0.10 & 0.03 & 0.12 & 0.24 & 97.44 \\
\hline 3.06 & 0.25 & 0.15 & 0.00 & 0.11 & 0.21 & 96.80 \\
\hline 3.06 & 0.27 & 0.09 & 0.03 & 0.11 & 0.17 & 96.90 \\
\hline 3.07 & 0.26 & 0.12 & 0.00 & 0.13 & 0.22 & 97.08 \\
\hline 3.10 & 0.27 & 0.11 & 0.05 & 0.12 & 0.19 & 97.17 \\
\hline 3.19 & 0.26 & 0.14 & 0.00 & 0.13 & 0.22 & 97.04 \\
\hline 3.13 & 0.26 & 0.12 & 0.00 & 0.13 & 0.20 & 96.90 \\
\hline 3.07 & 0.27 & 0.11 & 0.08 & 0.13 & 0.21 & 96.62 \\
\hline
\end{tabular}




\begin{tabular}{|c|c|c|c|c|c|c|}
\hline 3.22 & 0.26 & 0.16 & 0.00 & 0.12 & 0.19 & 97.05 \\
\hline 2.80 & 0.21 & 0.10 & 0.08 & 0.08 & 0.19 & 97.59 \\
\hline 2.79 & 0.21 & 0.11 & 0.07 & 0.08 & 0.17 & 97.82 \\
\hline 2.46 & 0.21 & 0.11 & 0.08 & 0.09 & 0.22 & 96.71 \\
\hline 2.62 & 0.26 & 0.12 & 0.05 & 0.12 & 0.18 & 96.62 \\
\hline 2.85 & 0.21 & 0.09 & 0.05 & 0.09 & 0.18 & 96.81 \\
\hline 2.80 & 0.22 & 0.11 & 0.00 & 0.08 & 0.20 & 97.43 \\
\hline 2.66 & 0.21 & 0.12 & 0.00 & 0.09 & 0.18 & 97.59 \\
\hline 2.92 & 0.23 & 0.12 & 0.09 & 0.09 & 0.19 & 97.79 \\
\hline 2.81 & 0.24 & 0.13 & 0.05 & 0.08 & 0.18 & 97.55 \\
\hline 2.89 & 0.20 & 0.07 & 0.00 & 0.07 & 0.19 & 97.75 \\
\hline 2.82 & 0.21 & 0.15 & 0.00 & 0.09 & 0.23 & 97.54 \\
\hline 2.81 & 0.22 & 0.16 & 0.00 & 0.09 & 0.18 & 97.61 \\
\hline 2.78 & 0.21 & 0.14 & 0.00 & 0.08 & 0.21 & 96.91 \\
\hline 2.76 & 0.21 & 0.12 & 0.03 & 0.08 & 0.19 & 96.60 \\
\hline 2.83 & 0.21 & 0.14 & 0.00 & 0.08 & 0.22 & 96.92 \\
\hline 3.32 & 0.26 & & 0.02 & 0.16 & 0.20 & 97.05 \\
\hline 3.38 & 0.25 & & 0.08 & 0.13 & 0.20 & 96.35 \\
\hline 3.08 & 0.27 & & 0.00 & 0.13 & 0.16 & 96.60 \\
\hline 3.34 & 0.25 & & 0.04 & 0.13 & 0.15 & 96.18 \\
\hline 3.00 & 0.23 & & 0.00 & 0.13 & 0.20 & 97.15 \\
\hline 3.22 & 0.23 & & 0.00 & 0.15 & 0.14 & 96.25 \\
\hline 3.33 & 0.25 & & 0.11 & 0.16 & 0.18 & 97.35 \\
\hline 3.30 & 0.26 & & 0.09 & 0.14 & 0.16 & 97.06 \\
\hline 3.40 & 0.25 & & 0.00 & 0.14 & 0.20 & 97.38 \\
\hline 3.16 & 0.23 & & 0.00 & 0.14 & 0.19 & 96.47 \\
\hline 3.23 & 0.25 & & 0.15 & 0.14 & 0.19 & 96.93 \\
\hline 3.07 & 0.29 & & 0.05 & 0.17 & 0.17 & 95.89 \\
\hline 3.06 & 0.27 & & 0.00 & 0.13 & 0.19 & 96.48 \\
\hline 2.38 & 0.23 & & 0.00 & 0.11 & 0.18 & 97.61 \\
\hline 3.23 & 0.26 & & 0.05 & 0.14 & 0.16 & 97.24 \\
\hline 3.02 & 0.22 & & 0.00 & 0.09 & 0.17 & 97.48 \\
\hline 2.77 & 0.22 & & 0.00 & 0.10 & 0.20 & 97.62 \\
\hline 2.95 & 0.22 & & 0.13 & 0.10 & 0.21 & 97.20 \\
\hline 2.90 & 0.20 & & 0.00 & 0.11 & 0.20 & 97.02 \\
\hline 2.90 & 0.21 & & 0.00 & 0.10 & 0.17 & 98.01 \\
\hline 2.94 & 0.21 & & 0.00 & 0.10 & 0.20 & 97.68 \\
\hline 2.91 & 0.21 & & 0.00 & 0.09 & 0.20 & 97.65 \\
\hline 2.99 & 0.21 & & 0.14 & 0.09 & 0.20 & 98.32 \\
\hline 2.82 & 0.21 & & 0.00 & 0.10 & 0.24 & 97.67 \\
\hline 2.98 & 0.22 & & 0.09 & 0.10 & 0.19 & 98.22 \\
\hline 2.94 & 0.21 & & 0.00 & 0.09 & 0.19 & 97.92 \\
\hline 2.84 & 0.21 & & 0.00 & 0.09 & 0.18 & 97.09 \\
\hline 2.95 & 0.20 & & 0.00 & 0.09 & 0.18 & 98.02 \\
\hline 2.93 & 0.20 & & 0.00 & 0.08 & 0.21 & 97.99 \\
\hline 2.99 & 0.21 & & 0.03 & 0.10 & 0.20 & 97.87 \\
\hline 3.01 & 0.22 & & 0.00 & 0.09 & 0.19 & 98.07 \\
\hline
\end{tabular}




\begin{tabular}{|c|c|c|c|c|c|c|}
\hline 2.83 & 0.21 & & 0.00 & 0.09 & 0.20 & 97.93 \\
\hline 2.94 & 0.22 & & 0.03 & 0.09 & 0.23 & 97.93 \\
\hline 2.89 & 0.20 & & 0.00 & 0.10 & 0.23 & 97.18 \\
\hline 2.98 & 0.21 & & 0.00 & 0.09 & 0.18 & 97.85 \\
\hline 2.61 & 0.23 & & 0.00 & 0.10 & 0.17 & 96.97 \\
\hline 2.98 & 0.22 & & 0.08 & 0.08 & 0.17 & 97.50 \\
\hline 2.90 & 0.21 & & 0.07 & 0.09 & 0.18 & 97.82 \\
\hline 2.94 & 0.22 & & 0.00 & 0.07 & 0.17 & 97.36 \\
\hline 2.95 & 0.21 & & 0.01 & 0.10 & 0.16 & 97.39 \\
\hline 2.90 & 0.21 & & 0.00 & 0.10 & 0.21 & 98.31 \\
\hline 2.99 & 0.21 & & 0.06 & 0.10 & 0.22 & 97.37 \\
\hline 3.01 & 0.21 & & 0.06 & 0.10 & 0.15 & 97.64 \\
\hline 2.87 & 0.21 & & 0.00 & 0.09 & 0.20 & 96.90 \\
\hline 2.96 & 0.21 & & 0.00 & 0.09 & 0.21 & 97.91 \\
\hline 2.89 & 0.19 & & 0.06 & 0.10 & 0.17 & 98.04 \\
\hline 2.89 & 0.22 & & 0.03 & 0.09 & 0.16 & 97.74 \\
\hline 2.87 & 0.23 & & 0.00 & 0.09 & 0.18 & 97.83 \\
\hline 2.93 & 0.21 & & 0.09 & 0.08 & 0.17 & 97.78 \\
\hline 2.95 & 0.20 & & 0.00 & 0.09 & 0.18 & 97.56 \\
\hline 3.00 & 0.22 & & 0.06 & 0.10 & 0.18 & 97.76 \\
\hline 2.86 & 0.23 & & 0.00 & 0.10 & 0.18 & 97.86 \\
\hline 2.93 & 0.20 & & 0.00 & 0.10 & 0.15 & 97.30 \\
\hline 2.93 & 0.22 & & 0.00 & 0.10 & 0.17 & 97.14 \\
\hline 2.79 & 0.23 & & 0.00 & 0.09 & 0.22 & 97.39 \\
\hline $\mathrm{Na} 2 \mathrm{O}$ & $\mathrm{K} 2 \mathrm{O}$ & P2O5 & $\mathrm{F}$ & $\mathrm{Cl}$ & $\mathrm{SO} 3$ & Total \\
\hline 3.18 & 0.27 & 0.17 & 0.00 & 0.16 & 0.22 & 96.72 \\
\hline 3.21 & 0.25 & 0.15 & 0.00 & 0.14 & 0.20 & 97.06 \\
\hline 3.06 & 0.24 & 0.16 & 0.00 & 0.14 & 0.20 & 97.62 \\
\hline 3.18 & 0.25 & 0.15 & 0.02 & 0.13 & 0.19 & 96.87 \\
\hline 3.22 & 0.26 & 0.14 & 0.00 & 0.15 & 0.20 & 97.37 \\
\hline 3.15 & 0.26 & 0.15 & 0.00 & 0.14 & 0.20 & 96.95 \\
\hline 3.02 & 0.25 & 0.14 & 0.07 & 0.12 & 0.18 & 97.34 \\
\hline 3.01 & 0.25 & 0.13 & 0.01 & 0.12 & 0.20 & 97.27 \\
\hline 3.26 & 0.25 & 0.10 & 0.05 & 0.13 & 0.17 & 96.73 \\
\hline 3.31 & 0.25 & 0.15 & 0.00 & 0.16 & 0.19 & 97.55 \\
\hline 2.69 & 0.22 & 0.09 & 0.01 & 0.11 & 0.17 & 98.22 \\
\hline 3.09 & 0.27 & 0.12 & 0.06 & 0.15 & 0.21 & 97.14 \\
\hline 3.15 & 0.26 & 0.11 & 0.22 & 0.15 & 0.18 & 97.34 \\
\hline 3.15 & 0.24 & 0.07 & 0.04 & 0.14 & 0.21 & 97.38 \\
\hline 3.18 & 0.24 & 0.15 & 0.14 & 0.13 & 0.16 & 97.50 \\
\hline 2.75 & 0.18 & 0.09 & 0.04 & 0.07 & 0.15 & 98.08 \\
\hline 2.71 & 0.18 & 0.15 & 0.03 & 0.08 & 0.17 & 98.16 \\
\hline 2.87 & 0.19 & 0.12 & 0.02 & 0.10 & 0.20 & 98.24 \\
\hline 2.84 & 0.19 & 0.09 & 0.00 & 0.10 & 0.18 & 98.15 \\
\hline 2.86 & 0.20 & 0.09 & 0.13 & 0.08 & 0.21 & 98.10 \\
\hline 2.76 & 0.18 & 0.09 & 0.00 & 0.08 & 0.16 & 97.55 \\
\hline
\end{tabular}




\begin{tabular}{|c|c|c|c|c|c|c|}
\hline 2.88 & 0.17 & 0.10 & 0.00 & 0.09 & 0.15 & 97.98 \\
\hline 2.85 & 0.18 & 0.08 & 0.06 & 0.09 & 0.15 & 98.14 \\
\hline 2.90 & 0.18 & 0.11 & 0.00 & 0.08 & 0.22 & 98.27 \\
\hline 2.79 & 0.18 & 0.11 & 0.02 & 0.09 & 0.17 & 97.52 \\
\hline 2.85 & 0.19 & 0.12 & 0.00 & 0.08 & 0.17 & 98.00 \\
\hline 2.78 & 0.18 & 0.11 & 0.00 & 0.09 & 0.19 & 97.21 \\
\hline 2.81 & 0.19 & 0.11 & 0.02 & 0.07 & 0.18 & 97.82 \\
\hline 2.67 & 0.18 & 0.10 & 0.09 & 0.07 & 0.20 & 97.23 \\
\hline 2.76 & 0.18 & 0.07 & 0.00 & 0.09 & 0.18 & 97.26 \\
\hline 2.84 & 0.19 & 0.08 & 0.00 & 0.07 & 0.19 & 98.38 \\
\hline 2.77 & 0.19 & 0.13 & 0.04 & 0.09 & 0.19 & 97.92 \\
\hline 2.91 & 0.19 & 0.15 & 0.00 & 0.07 & 0.17 & 98.50 \\
\hline 2.79 & 0.18 & 0.10 & 0.00 & 0.09 & 0.19 & 98.23 \\
\hline 2.82 & 0.18 & 0.12 & 0.00 & 0.09 & 0.18 & 97.49 \\
\hline 2.86 & 0.18 & 0.06 & 0.06 & 0.09 & 0.22 & 98.26 \\
\hline 2.80 & 0.21 & 0.12 & 0.00 & 0.08 & 0.18 & 97.83 \\
\hline 2.74 & 0.19 & 0.10 & 0.00 & 0.09 & 0.20 & 97.96 \\
\hline 2.85 & 0.18 & 0.14 & 0.00 & 0.08 & 0.20 & 98.42 \\
\hline 2.81 & 0.20 & 0.08 & 0.01 & 0.09 & 0.18 & 97.32 \\
\hline 2.85 & 0.19 & 0.08 & 0.00 & 0.08 & 0.20 & 97.72 \\
\hline 2.80 & 0.19 & 0.08 & 0.01 & 0.09 & 0.16 & 97.85 \\
\hline 2.86 & 0.19 & 0.09 & 0.00 & 0.07 & 0.17 & 97.71 \\
\hline 2.70 & 0.19 & 0.09 & 0.00 & 0.08 & 0.19 & 98.08 \\
\hline 2.80 & 0.18 & 0.10 & 0.17 & 0.09 & 0.19 & 97.87 \\
\hline 3.18 & 0.28 & 0.11 & 0.00 & 0.15 & 0.11 & 96.49 \\
\hline 3.22 & 0.25 & 0.09 & 0.00 & 0.16 & 0.15 & 96.90 \\
\hline 2.76 & 0.29 & 0.06 & 0.00 & 0.17 & 0.13 & 95.76 \\
\hline 3.25 & 0.26 & 0.11 & 0.00 & 0.17 & 0.18 & 96.63 \\
\hline 3.25 & 0.27 & 0.11 & 0.04 & 0.17 & 0.15 & 97.05 \\
\hline 2.56 & 0.24 & 0.16 & 0.00 & 0.15 & 0.37 & 94.18 \\
\hline 3.09 & 0.26 & 0.14 & 0.00 & 0.15 & 0.16 & 96.75 \\
\hline 3.10 & 0.26 & 0.15 & 0.00 & 0.17 & 0.16 & 97.15 \\
\hline 3.15 & 0.28 & 0.11 & 0.01 & 0.15 & 0.16 & 96.85 \\
\hline 3.16 & 0.24 & 0.15 & 0.00 & 0.15 & 0.15 & 96.89 \\
\hline 3.12 & 0.26 & 0.09 & 0.00 & 0.17 & 0.13 & 97.00 \\
\hline 3.24 & 0.26 & 0.14 & 0.00 & 0.16 & 0.13 & 96.98 \\
\hline 3.23 & 0.25 & 0.14 & 0.00 & 0.16 & 0.15 & 96.48 \\
\hline 3.16 & 0.25 & 0.16 & 0.08 & 0.14 & 0.22 & 97.31 \\
\hline 3.03 & 0.25 & 0.14 & 0.05 & 0.12 & 0.17 & 97.45 \\
\hline 3.15 & 0.27 & 0.17 & 0.00 & 0.12 & 0.20 & 97.72 \\
\hline 3.17 & 0.27 & 0.11 & 0.00 & 0.13 & 0.19 & 97.44 \\
\hline 3.17 & 0.26 & 0.14 & 0.00 & 0.14 & 0.19 & 97.41 \\
\hline 3.16 & 0.26 & 0.11 & 0.00 & 0.13 & 0.24 & 97.58 \\
\hline 2.84 & 0.39 & 0.14 & 0.00 & 0.15 & 0.21 & 95.53 \\
\hline 3.06 & 0.27 & 0.09 & 0.00 & 0.13 & 0.19 & 97.40 \\
\hline 3.26 & 0.26 & 0.13 & 0.00 & 0.14 & 0.19 & 97.70 \\
\hline 3.18 & 0.26 & 0.15 & 0.00 & 0.13 & 0.20 & 97.55 \\
\hline
\end{tabular}




\begin{tabular}{|c|c|c|c|c|c|c|}
\hline 3.03 & 0.23 & 0.12 & 0.00 & 0.12 & 0.19 & 97.53 \\
\hline 3.22 & 0.24 & 0.15 & 0.07 & 0.11 & 0.21 & 97.19 \\
\hline 3.04 & 0.26 & 0.10 & 0.00 & 0.11 & 0.19 & 97.05 \\
\hline 3.19 & 0.25 & 0.15 & 0.00 & 0.12 & 0.19 & 97.59 \\
\hline 3.07 & 0.26 & 0.13 & 0.10 & 0.13 & 0.22 & 97.33 \\
\hline 3.19 & 0.25 & 0.11 & 0.00 & 0.12 & 0.20 & 97.49 \\
\hline 3.16 & 0.26 & 0.13 & 0.02 & 0.13 & 0.18 & 97.59 \\
\hline 3.30 & 0.26 & 0.13 & 0.02 & 0.12 & 0.17 & 97.44 \\
\hline 3.07 & 0.26 & 0.10 & 0.07 & 0.13 & 0.17 & 97.56 \\
\hline 3.13 & 0.25 & 0.08 & 0.00 & 0.15 & 0.16 & 96.59 \\
\hline 3.05 & 0.24 & 0.10 & 0.00 & 0.13 & 0.21 & 96.79 \\
\hline 3.04 & 0.26 & 0.14 & 0.00 & 0.14 & 0.19 & 97.18 \\
\hline 2.91 & 0.24 & 0.14 & 0.00 & 0.14 & 0.18 & 96.45 \\
\hline 3.20 & 0.25 & 0.13 & 0.00 & 0.14 & 0.22 & 97.33 \\
\hline 3.19 & 0.26 & 0.15 & 0.00 & 0.12 & 0.17 & 97.67 \\
\hline 2.95 & 0.25 & 0.14 & 0.00 & 0.14 & 0.20 & 97.39 \\
\hline 3.14 & 0.25 & 0.14 & 0.10 & 0.14 & 0.20 & 96.82 \\
\hline 3.02 & 0.25 & 0.11 & 0.00 & 0.12 & 0.17 & 96.85 \\
\hline 3.17 & 0.24 & 0.13 & 0.02 & 0.11 & 0.19 & 97.82 \\
\hline 3.27 & 0.25 & & 0.00 & 0.14 & 0.18 & 96.93 \\
\hline 3.21 & 0.26 & & 0.01 & 0.16 & 0.17 & 97.22 \\
\hline 3.28 & 0.24 & & 0.00 & 0.16 & 0.16 & 97.51 \\
\hline 3.28 & 0.25 & & 0.17 & 0.13 & 0.21 & 97.36 \\
\hline 3.31 & 0.25 & & 0.00 & 0.12 & 0.21 & 97.32 \\
\hline 3.32 & 0.24 & & 0.00 & 0.13 & 0.16 & 97.37 \\
\hline 3.31 & 0.26 & & 0.00 & 0.14 & 0.20 & 96.48 \\
\hline 3.33 & 0.24 & & 0.00 & 0.14 & 0.19 & 97.17 \\
\hline 3.09 & 0.26 & & 0.00 & 0.14 & 0.19 & 97.11 \\
\hline 3.30 & 0.23 & & 0.00 & 0.14 & 0.16 & 97.03 \\
\hline 3.38 & 0.25 & & 0.14 & 0.22 & 0.18 & 96.47 \\
\hline 3.24 & 0.28 & & 0.00 & 0.23 & 0.15 & 96.60 \\
\hline 3.49 & 0.27 & & 0.07 & 0.19 & 0.18 & 96.75 \\
\hline 3.37 & 0.27 & & 0.00 & 0.20 & 0.17 & 97.03 \\
\hline 2.99 & 0.25 & & 0.00 & 0.19 & 0.18 & 96.51 \\
\hline 3.35 & 0.27 & & 0.00 & 0.18 & 0.19 & 96.98 \\
\hline 3.28 & 0.25 & & 0.00 & 0.18 & 0.16 & 96.79 \\
\hline 3.25 & 0.25 & & 0.00 & 0.16 & 0.18 & 96.27 \\
\hline 3.23 & 0.25 & & 0.00 & 0.16 & 0.18 & 96.58 \\
\hline 3.29 & 0.26 & & 0.02 & 0.19 & 0.17 & 96.89 \\
\hline 2.91 & 0.18 & & 0.10 & 0.10 & 0.21 & 97.99 \\
\hline 2.87 & 0.17 & & 0.03 & 0.08 & 0.21 & 97.49 \\
\hline 2.76 & 0.19 & & 0.14 & 0.07 & 0.18 & 97.96 \\
\hline 2.64 & 0.18 & & 0.00 & 0.09 & 0.19 & 97.49 \\
\hline 2.74 & 0.17 & & 0.12 & 0.08 & 0.19 & 97.53 \\
\hline 2.82 & 0.19 & & 0.00 & 0.08 & 0.17 & 97.60 \\
\hline 2.92 & 0.17 & & 0.06 & 0.08 & 0.22 & 97.93 \\
\hline 2.64 & 0.18 & & 0.00 & 0.09 & 0.17 & 97.82 \\
\hline
\end{tabular}




\begin{tabular}{|c|c|c|c|c|c|c|}
\hline 2.94 & 0.18 & & 0.00 & 0.09 & 0.19 & 98.15 \\
\hline 3.32 & 0.25 & & 0.07 & 0.16 & 0.16 & 97.16 \\
\hline 3.34 & 0.27 & & 0.00 & 0.18 & 0.15 & 96.98 \\
\hline 3.26 & 0.25 & & 0.03 & 0.17 & 0.14 & 96.82 \\
\hline 3.26 & 0.26 & & 0.05 & 0.16 & 0.16 & 97.11 \\
\hline 3.35 & 0.25 & & 0.00 & 0.17 & 0.15 & 96.92 \\
\hline 3.26 & 0.25 & & 0.06 & 0.15 & 0.16 & 96.55 \\
\hline 3.31 & 0.27 & & 0.13 & 0.16 & 0.16 & 96.00 \\
\hline 3.19 & 0.24 & & 0.00 & 0.17 & 0.17 & 95.94 \\
\hline 3.17 & 0.26 & & 0.00 & 0.18 & 0.17 & 96.81 \\
\hline 3.39 & 0.26 & & 0.00 & 0.16 & 0.20 & 97.04 \\
\hline 3.30 & 0.25 & & 0.01 & 0.13 & 0.24 & 97.91 \\
\hline 3.32 & 0.26 & & 0.00 & 0.12 & 0.21 & 97.04 \\
\hline 3.14 & 0.26 & & 0.00 & 0.15 & 0.20 & 97.63 \\
\hline 3.33 & 0.25 & & 0.00 & 0.15 & 0.22 & 97.40 \\
\hline 3.20 & 0.26 & & 0.09 & 0.14 & 0.21 & 97.17 \\
\hline 3.32 & 0.24 & & 0.00 & 0.13 & 0.19 & 96.91 \\
\hline 3.29 & 0.27 & & 0.00 & 0.14 & 0.18 & 97.25 \\
\hline 3.19 & 0.25 & & 0.00 & 0.13 & 0.22 & 97.05 \\
\hline 3.32 & 0.25 & & 0.00 & 0.13 & 0.19 & 97.02 \\
\hline 2.97 & 0.24 & & 0.05 & 0.12 & 0.15 & 98.14 \\
\hline $\mathrm{Na} 2 \mathrm{O}$ & $\mathrm{K} 2 \mathrm{O}$ & P2O5 & $\mathrm{F}$ & $\mathrm{Cl}$ & $\mathrm{SO} 3$ & Total \\
\hline 3.05 & 0.27 & 0.11 & 0.08 & 0.13 & 0.22 & 97.68 \\
\hline 3.18 & 0.25 & 0.08 & 0.06 & 0.11 & 0.20 & 97.84 \\
\hline 3.07 & 0.26 & 0.09 & 0.00 & 0.12 & 0.17 & 97.68 \\
\hline 3.18 & 0.25 & 0.12 & 0.00 & 0.12 & 0.17 & 97.68 \\
\hline 3.20 & 0.26 & 0.15 & 0.00 & 0.12 & 0.19 & 97.83 \\
\hline 3.02 & 0.24 & 0.12 & 0.00 & 0.14 & 0.21 & 97.84 \\
\hline 3.16 & 0.26 & 0.08 & 0.00 & 0.11 & 0.17 & 97.46 \\
\hline 3.16 & 0.26 & 0.18 & 0.03 & 0.12 & 0.19 & 97.81 \\
\hline 3.14 & 0.26 & 0.12 & 0.00 & 0.14 & 0.18 & 97.75 \\
\hline 3.15 & 0.24 & 0.08 & 0.00 & 0.13 & 0.19 & 97.98 \\
\hline 3.23 & 0.24 & 0.15 & 0.03 & 0.12 & 0.18 & 97.64 \\
\hline 3.13 & 0.27 & 0.12 & 0.14 & 0.12 & 0.19 & 97.93 \\
\hline 3.26 & 0.26 & 0.09 & 0.04 & 0.12 & 0.22 & 97.95 \\
\hline 3.20 & 0.24 & 0.14 & 0.06 & 0.12 & 0.20 & 98.00 \\
\hline 3.15 & 0.27 & 0.17 & 0.00 & 0.12 & 0.18 & 98.14 \\
\hline 3.22 & 0.25 & 0.16 & 0.00 & 0.13 & 0.17 & 98.23 \\
\hline 3.29 & 0.26 & 0.12 & 0.00 & 0.15 & 0.18 & 97.79 \\
\hline 3.20 & 0.25 & 0.09 & 0.18 & 0.12 & 0.23 & 98.47 \\
\hline 3.23 & 0.26 & 0.09 & 0.15 & 0.13 & 0.21 & 97.93 \\
\hline 2.89 & 0.27 & 0.12 & 0.00 & 0.14 & 0.21 & 97.61 \\
\hline 3.26 & 0.26 & 0.11 & 0.03 & 0.14 & 0.20 & 98.06 \\
\hline 3.19 & 0.27 & 0.15 & 0.00 & 0.14 & 0.20 & 97.07 \\
\hline 3.16 & 0.27 & 0.13 & 0.00 & 0.13 & 0.22 & 98.04 \\
\hline 2.96 & 0.27 & 0.13 & 0.11 & 0.13 & 0.19 & 97.92 \\
\hline
\end{tabular}




\begin{tabular}{|c|c|c|c|c|c|c|}
\hline 3.24 & 0.27 & 0.12 & 0.00 & 0.14 & 0.20 & 98.22 \\
\hline 3.16 & 0.26 & 0.10 & 0.10 & 0.13 & 0.18 & 98.37 \\
\hline 3.17 & 0.28 & 0.16 & 0.04 & 0.13 & 0.20 & 97.49 \\
\hline 3.21 & 0.27 & 0.13 & 0.00 & 0.14 & 0.17 & 97.84 \\
\hline 3.20 & 0.27 & 0.11 & 0.05 & 0.13 & 0.19 & 97.77 \\
\hline 3.24 & 0.27 & 0.14 & 0.00 & 0.14 & 0.23 & 98.27 \\
\hline 3.24 & 0.26 & 0.18 & 0.00 & 0.11 & 0.20 & 97.48 \\
\hline 3.15 & 0.26 & 0.11 & 0.00 & 0.11 & 0.17 & 97.78 \\
\hline 3.15 & 0.25 & 0.16 & 0.04 & 0.13 & 0.20 & 98.55 \\
\hline 3.10 & 0.26 & 0.15 & 0.14 & 0.13 & 0.20 & 98.07 \\
\hline 3.11 & 0.27 & 0.13 & 0.00 & 0.13 & 0.20 & 97.83 \\
\hline 3.15 & 0.28 & 0.13 & 0.08 & 0.13 & 0.20 & 98.02 \\
\hline 3.21 & 0.24 & 0.14 & 0.00 & 0.12 & 0.20 & 98.25 \\
\hline 3.08 & 0.26 & 0.13 & 0.00 & 0.12 & 0.20 & 98.09 \\
\hline 3.22 & 0.27 & 0.10 & 0.01 & 0.10 & 0.21 & 98.19 \\
\hline 3.25 & 0.26 & 0.17 & 0.00 & 0.13 & 0.21 & 98.29 \\
\hline 3.13 & 0.26 & 0.13 & 0.00 & 0.12 & 0.21 & 98.30 \\
\hline 3.17 & 0.25 & 0.14 & 0.03 & 0.12 & 0.19 & 98.27 \\
\hline 3.24 & 0.25 & 0.14 & 0.01 & 0.11 & 0.20 & 97.52 \\
\hline 3.31 & 0.28 & 0.07 & 0.00 & 0.13 & 0.21 & 96.42 \\
\hline 3.16 & 0.27 & 0.09 & 0.00 & 0.13 & 0.19 & 97.78 \\
\hline 3.22 & 0.27 & 0.15 & 0.00 & 0.13 & 0.18 & 97.79 \\
\hline 3.26 & 0.20 & 0.10 & 0.04 & 0.10 & 0.14 & 98.44 \\
\hline 3.22 & 0.26 & 0.09 & 0.07 & 0.12 & 0.20 & 97.64 \\
\hline 3.00 & 0.27 & 0.13 & 0.00 & 0.13 & 0.18 & 97.60 \\
\hline 3.16 & 0.25 & 0.13 & 0.01 & 0.13 & 0.19 & 97.92 \\
\hline 3.15 & 0.26 & 0.12 & 0.00 & 0.14 & 0.21 & 97.57 \\
\hline 3.14 & 0.27 & 0.13 & 0.00 & 0.12 & 0.16 & 97.36 \\
\hline 3.16 & 0.26 & 0.12 & 0.09 & 0.12 & 0.22 & 97.48 \\
\hline 3.14 & 0.26 & 0.14 & 0.09 & 0.13 & 0.20 & 97.90 \\
\hline 3.17 & 0.25 & 0.09 & 0.00 & 0.13 & 0.19 & 96.97 \\
\hline 3.05 & 0.27 & 0.10 & 0.00 & 0.13 & 0.20 & 97.30 \\
\hline 3.23 & 0.24 & 0.15 & 0.18 & 0.13 & 0.19 & 97.82 \\
\hline 3.15 & 0.27 & 0.07 & 0.00 & 0.13 & 0.19 & 97.73 \\
\hline 3.16 & 0.26 & 0.08 & 0.00 & 0.14 & 0.20 & 97.99 \\
\hline 3.21 & 0.26 & 0.15 & 0.05 & 0.13 & 0.20 & 97.88 \\
\hline 3.21 & 0.25 & 0.11 & 0.06 & 0.12 & 0.21 & 96.86 \\
\hline 3.27 & 0.27 & 0.11 & 0.00 & 0.12 & 0.19 & 97.93 \\
\hline 3.19 & 0.28 & 0.13 & 0.00 & 0.12 & 0.20 & 97.20 \\
\hline 3.16 & 0.25 & 0.10 & 0.10 & 0.10 & 0.22 & 98.34 \\
\hline 3.28 & 0.26 & 0.14 & 0.06 & 0.12 & 0.18 & 97.76 \\
\hline 3.23 & 0.24 & 0.11 & 0.00 & 0.13 & 0.19 & 97.71 \\
\hline 3.18 & 0.27 & 0.12 & 0.04 & 0.12 & 0.23 & 98.01 \\
\hline 3.19 & 0.27 & 0.15 & 0.00 & 0.14 & 0.18 & 97.58 \\
\hline 3.25 & 0.27 & 0.14 & 0.08 & 0.13 & 0.19 & 97.86 \\
\hline 3.14 & 0.26 & 0.09 & 0.00 & 0.13 & 0.19 & 98.02 \\
\hline 3.19 & 0.25 & 0.09 & 0.03 & 0.11 & 0.17 & 98.00 \\
\hline
\end{tabular}




\begin{tabular}{|c|c|c|c|c|c|c|}
\hline 3.08 & 0.26 & 0.07 & 0.00 & 0.12 & 0.20 & 98.20 \\
\hline 3.24 & 0.26 & 0.13 & 0.02 & 0.15 & 0.21 & 97.90 \\
\hline 2.88 & 0.26 & 0.13 & 0.05 & 0.13 & 0.21 & 98.10 \\
\hline 3.12 & 0.26 & 0.10 & 0.16 & 0.13 & 0.19 & 97.93 \\
\hline 3.15 & 0.25 & 0.13 & 0.00 & 0.11 & 0.20 & 97.79 \\
\hline 3.29 & 0.26 & 0.14 & 0.00 & 0.12 & 0.20 & 97.83 \\
\hline 3.25 & 0.28 & 0.08 & 0.02 & 0.13 & 0.17 & 97.86 \\
\hline 3.28 & 0.28 & 0.16 & 0.12 & 0.14 & 0.21 & 98.16 \\
\hline 3.21 & 0.28 & 0.13 & 0.00 & 0.13 & 0.20 & 97.19 \\
\hline 3.25 & 0.27 & 0.15 & 0.04 & 0.11 & 0.18 & 97.71 \\
\hline 3.12 & 0.24 & 0.14 & 0.00 & 0.13 & 0.24 & 97.80 \\
\hline 3.20 & 0.26 & 0.06 & 0.00 & 0.13 & 0.18 & 98.30 \\
\hline 3.13 & 0.27 & 0.13 & 0.00 & 0.13 & 0.16 & 97.16 \\
\hline 2.87 & 0.24 & 0.09 & 0.00 & 0.10 & 0.20 & 98.15 \\
\hline 3.21 & 0.25 & 0.11 & 0.00 & 0.13 & 0.21 & 97.70 \\
\hline 3.29 & 0.25 & & 0.00 & 0.13 & 0.19 & 97.89 \\
\hline 3.15 & 0.27 & & 0.00 & 0.13 & 0.24 & 97.66 \\
\hline 3.21 & 0.25 & & 0.00 & 0.13 & 0.20 & 97.55 \\
\hline 3.36 & 0.25 & & 0.00 & 0.13 & 0.19 & 97.71 \\
\hline 3.30 & 0.21 & & 0.00 & 0.10 & 0.19 & 98.57 \\
\hline 3.33 & 0.24 & & 0.00 & 0.14 & 0.19 & 97.92 \\
\hline 3.34 & 0.28 & & 0.00 & 0.13 & 0.18 & 97.94 \\
\hline 3.22 & 0.28 & & 0.00 & 0.13 & 0.17 & 97.90 \\
\hline 3.30 & 0.24 & & 0.15 & 0.13 & 0.23 & 98.39 \\
\hline 3.15 & 0.26 & & 0.08 & 0.13 & 0.24 & 98.42 \\
\hline 3.35 & 0.24 & & 0.00 & 0.15 & 0.20 & 98.27 \\
\hline 3.32 & 0.24 & & 0.02 & 0.12 & 0.19 & 98.61 \\
\hline 3.26 & 0.26 & & 0.00 & 0.15 & 0.21 & 97.30 \\
\hline 3.21 & 0.26 & & 0.07 & 0.15 & 0.20 & 97.06 \\
\hline 3.45 & 0.25 & & 0.03 & 0.15 & 0.21 & 97.84 \\
\hline 3.22 & 0.27 & & 0.03 & 0.13 & 0.20 & 97.17 \\
\hline 3.35 & 0.28 & & 0.01 & 0.15 & 0.22 & 97.73 \\
\hline 3.31 & 0.26 & & 0.00 & 0.13 & 0.23 & 97.64 \\
\hline 3.29 & 0.27 & & 0.00 & 0.13 & 0.21 & 96.00 \\
\hline 3.25 & 0.26 & & 0.03 & 0.14 & 0.20 & 97.73 \\
\hline 3.27 & 0.24 & & 0.00 & 0.14 & 0.23 & 98.13 \\
\hline 3.23 & 0.26 & & 0.00 & 0.15 & 0.19 & 97.82 \\
\hline 3.21 & 0.25 & & 0.00 & 0.12 & 0.18 & 98.00 \\
\hline 3.16 & 0.26 & & 0.04 & 0.14 & 0.21 & 97.51 \\
\hline 3.26 & 0.25 & & 0.00 & 0.13 & 0.21 & 97.10 \\
\hline 3.26 & 0.27 & & 0.09 & 0.13 & 0.20 & 98.03 \\
\hline 3.02 & 0.27 & & 0.00 & 0.14 & 0.20 & 97.37 \\
\hline 3.31 & 0.26 & & 0.00 & 0.13 & 0.18 & 97.78 \\
\hline 3.19 & 0.25 & & 0.17 & 0.13 & 0.17 & 98.61 \\
\hline 3.33 & 0.26 & & 0.08 & 0.14 & 0.20 & 98.02 \\
\hline 3.37 & 0.28 & & 0.11 & 0.17 & 0.16 & 97.74 \\
\hline 3.13 & 0.25 & & 0.00 & 0.16 & 0.16 & 97.45 \\
\hline
\end{tabular}




$\begin{array}{llllll}3.20 & 0.26 & 0.00 & 0.16 & 0.18 & 97.68 \\ 3.11 & 0.24 & 0.00 & 0.15 & 0.15 & 97.40 \\ 3.29 & 0.25 & 0.00 & 0.15 & 0.17 & 97.68 \\ 3.33 & 0.24 & 0.02 & 0.15 & 0.17 & 98.23 \\ 3.40 & 0.25 & 0.00 & 0.15 & 0.19 & 97.90 \\ 3.27 & 0.26 & 0.02 & 0.16 & 0.18 & 97.54\end{array}$




\begin{tabular}{|c|c|c|c|c|c|c|c|c|}
\hline R05 & $\mathrm{SiO} 2$ & $\mathrm{TiO} 2$ & Al2O3 & $\mathrm{Cr} 2 \mathrm{O} 3$ & $\mathrm{FeO}$ & $\mathrm{MnO}$ & $\mathrm{MgO}$ & $\mathrm{CaO}$ \\
\hline R05-12r & 50.89 & 1.00 & 16.60 & 0.00 & 8.21 & 0.16 & 6.43 & 11.50 \\
\hline R05-12r & 50.96 & 1.03 & 16.53 & 0.00 & 8.13 & 0.13 & 6.49 & 11.38 \\
\hline R05-12r & 50.44 & 0.97 & 16.51 & 0.00 & 8.00 & 0.15 & 6.43 & 11.45 \\
\hline R05-12r & 50.52 & 0.96 & 15.76 & 0.02 & 8.16 & 0.15 & 6.39 & 11.26 \\
\hline R05-12r & 50.92 & 0.99 & 16.52 & 0.02 & 8.18 & 0.16 & 6.34 & 11.28 \\
\hline R05-12r & 50.90 & 0.95 & 16.53 & 0.02 & 8.01 & 0.13 & 6.37 & 11.30 \\
\hline R05-12r & 50.89 & 0.99 & 16.48 & 0.03 & 7.96 & 0.13 & 6.36 & 11.36 \\
\hline R05-12r & 51.02 & 0.98 & 16.55 & 0.00 & 8.13 & 0.16 & 6.38 & 11.38 \\
\hline R05-12r & 50.81 & 0.99 & 16.56 & 0.00 & 8.19 & 0.16 & 6.14 & 11.24 \\
\hline R05-12r & 50.92 & 0.95 & 16.49 & 0.00 & 8.09 & 0.18 & 6.36 & 11.17 \\
\hline R05-12r & 50.63 & 0.99 & 16.57 & 0.03 & 7.87 & 0.15 & 6.42 & 11.28 \\
\hline R05-12r & 50.77 & 0.96 & 16.27 & 0.02 & 8.14 & 0.13 & 6.12 & 11.33 \\
\hline R05-12r & 50.82 & 1.00 & 16.60 & 0.01 & 8.18 & 0.16 & 6.37 & 11.34 \\
\hline R05-12r & 50.88 & 1.00 & 16.06 & 0.00 & 8.11 & 0.12 & 6.87 & 11.85 \\
\hline R05-12r & 50.83 & 1.02 & 16.51 & 0.01 & 8.18 & 0.15 & 6.43 & 11.33 \\
\hline R05-12r & 50.81 & 0.97 & 16.49 & 0.01 & 7.93 & 0.16 & 6.35 & 11.37 \\
\hline R05-12r & 50.96 & 0.99 & 16.56 & 0.01 & 8.20 & 0.16 & 6.18 & 11.32 \\
\hline R05-12r & 50.92 & 0.99 & 16.53 & 0.01 & 8.12 & 0.13 & 6.37 & 11.23 \\
\hline R05-12r & 50.80 & 0.94 & 16.49 & 0.00 & 8.12 & 0.15 & 6.31 & 11.21 \\
\hline R05-12r & 50.84 & 0.97 & 16.49 & 0.02 & 8.18 & 0.13 & 6.42 & 11.33 \\
\hline R05-12r & 50.94 & 1.00 & 16.42 & 0.01 & 8.14 & 0.16 & 6.36 & 11.15 \\
\hline R05-12r & 50.68 & 1.01 & 16.43 & 0.01 & 7.94 & 0.16 & 6.33 & 11.07 \\
\hline R05-12r & 50.92 & 1.00 & 16.57 & 0.00 & 8.21 & 0.14 & 6.39 & 11.30 \\
\hline R05-12r & 50.81 & 0.96 & 16.45 & 0.00 & 8.04 & 0.15 & 6.18 & 11.37 \\
\hline R05-12r & 51.06 & 0.99 & 16.53 & 0.03 & 8.18 & 0.16 & 6.33 & 11.42 \\
\hline R05-12r & 50.94 & 0.98 & 16.52 & 0.01 & 8.16 & 0.12 & 6.34 & 11.15 \\
\hline R05-12r & 50.91 & 1.00 & 16.40 & 0.01 & 7.99 & 0.15 & 6.27 & 11.45 \\
\hline R05-12r & 51.09 & 0.96 & 16.41 & 0.01 & 8.06 & 0.16 & 6.31 & 11.27 \\
\hline R05-12r & 51.01 & 0.97 & 16.58 & 0.02 & 8.14 & 0.17 & 6.34 & 11.35 \\
\hline R05-12r & 51.04 & 1.02 & 16.61 & 0.00 & 8.11 & 0.14 & 6.40 & 11.43 \\
\hline R05-12r & 50.85 & 0.98 & 16.53 & 0.01 & 8.14 & 0.11 & 6.25 & 11.29 \\
\hline R05-12r & 51.18 & 0.93 & 16.61 & 0.02 & 8.24 & 0.18 & 6.38 & 11.26 \\
\hline R05-12r & 50.69 & 0.98 & 16.51 & 0.00 & 8.10 & 0.14 & 6.29 & 11.34 \\
\hline R05-12r & 50.98 & 0.97 & 16.48 & 0.02 & 8.16 & 0.16 & 6.34 & 11.21 \\
\hline R05-12r & 51.09 & 0.95 & 16.52 & 0.01 & 8.07 & 0.12 & 6.31 & 11.03 \\
\hline R05-13r & 50.68 & 0.97 & 16.36 & 0.00 & 8.15 & 0.16 & 6.30 & 11.39 \\
\hline R05-13r & 50.64 & 0.99 & 16.11 & 0.02 & 8.04 & 0.15 & 6.31 & 11.33 \\
\hline R05-13r & 50.92 & 0.97 & 16.63 & 0.00 & 8.30 & 0.15 & 6.35 & 11.35 \\
\hline R05-13r & 50.24 & 1.04 & 16.55 & 0.00 & 8.20 & 0.17 & 6.32 & 11.37 \\
\hline R05-13r & 50.63 & 1.01 & 16.48 & 0.00 & 8.25 & 0.18 & 6.36 & 11.31 \\
\hline R05-13r & 50.66 & 0.98 & 16.48 & 0.01 & 8.24 & 0.16 & 6.26 & 11.33 \\
\hline R05-13r & 50.95 & 1.04 & 16.45 & 0.00 & 8.23 & 0.15 & 6.20 & 11.34 \\
\hline R05-13r & 50.88 & 1.00 & 16.30 & 0.04 & 8.28 & 0.19 & 6.38 & 11.19 \\
\hline R05-13r & 51.05 & 0.96 & 16.06 & 0.01 & 8.32 & 0.14 & 7.19 & 11.06 \\
\hline R05-13r & 51.01 & 1.00 & 16.47 & 0.00 & 8.16 & 0.15 & 6.48 & 11.36 \\
\hline R05-13r & 50.51 & 0.99 & 16.56 & 0.01 & 8.19 & 0.16 & 6.41 & 11.32 \\
\hline
\end{tabular}




\begin{tabular}{|c|c|c|c|c|c|c|c|c|}
\hline $\mathrm{R} 05-13 \mathrm{r}$ & 50.37 & 1.01 & 16.25 & 0.00 & 8.25 & 0.18 & 5.93 & 11.35 \\
\hline $\mathrm{R} 05-13 r$ & 51.01 & 0.99 & 16.43 & 0.00 & 7.45 & 0.12 & 6.30 & 11.32 \\
\hline R05-14r & 50.32 & 0.98 & 16.52 & 0.01 & 8.25 & 0.17 & 6.34 & 11.31 \\
\hline R05-14r & 50.69 & 1.02 & 16.51 & 0.02 & 8.26 & 0.15 & 6.27 & 11.38 \\
\hline$R 05-14 r$ & 51.01 & 0.95 & 16.28 & 0.00 & 8.12 & 0.16 & 6.24 & 10.97 \\
\hline$R 05-14 r$ & 50.94 & 0.96 & 16.57 & 0.01 & 7.85 & 0.18 & 6.33 & 11.22 \\
\hline$R 05-14 r$ & 50.97 & 0.99 & 16.38 & 0.00 & 8.06 & 0.12 & 6.25 & 11.35 \\
\hline$R 05-14 r$ & 50.90 & 0.96 & 16.24 & 0.02 & 8.27 & 0.15 & 6.32 & 11.13 \\
\hline$R 05-14 r$ & 50.97 & 0.97 & 16.42 & 0.00 & 8.21 & 0.14 & 6.03 & 11.11 \\
\hline$R 05-14 r$ & 51.06 & 1.05 & 16.51 & 0.01 & 8.12 & 0.15 & 6.42 & 11.17 \\
\hline $\mathrm{R} 05-14 \mathrm{r}$ & 51.01 & 0.94 & 16.08 & 0.01 & 7.77 & 0.16 & 6.28 & 11.31 \\
\hline$R 05-14 r$ & 50.92 & 0.96 & 16.38 & 0.03 & 8.22 & 0.14 & 6.32 & 11.15 \\
\hline$R 05-14 r$ & 51.05 & 1.02 & 16.48 & 0.01 & 8.22 & 0.14 & 6.32 & 11.26 \\
\hline$R 05-14 r$ & 50.74 & 0.93 & 15.53 & 0.02 & 8.50 & 0.15 & 7.72 & 10.91 \\
\hline R05-14r & 51.05 & 0.98 & 16.53 & 0.00 & 8.26 & 0.15 & 6.27 & 11.35 \\
\hline$R 05-14 r$ & 50.98 & 1.00 & 16.55 & 0.00 & 8.28 & 0.16 & 6.27 & 11.28 \\
\hline$R 05-14 r$ & 51.03 & 0.99 & 16.30 & 0.01 & 7.71 & 0.16 & 6.18 & 11.21 \\
\hline $\mathrm{R} 05-17 \mathrm{r}$ & 50.24 & 0.99 & 16.44 & 0.00 & 8.14 & 0.19 & 6.45 & 11.33 \\
\hline $\mathrm{R} 05-17 \mathrm{r}$ & 50.86 & 0.96 & 16.44 & 0.01 & 7.04 & 0.18 & 6.11 & 11.29 \\
\hline $\mathrm{R} 05-17 r$ & 50.83 & 1.00 & 16.32 & 0.00 & 8.16 & 0.18 & 6.34 & 10.99 \\
\hline $\mathrm{R} 05-17 r$ & 51.03 & 0.95 & 16.49 & 0.00 & 7.95 & 0.16 & 6.32 & 11.34 \\
\hline $\mathrm{R} 05-17 \mathrm{r}$ & 51.07 & 0.98 & 16.50 & 0.01 & 8.13 & 0.17 & 6.35 & 11.36 \\
\hline $\mathrm{R} 05-17 \mathrm{r}$ & 50.77 & 0.96 & 16.45 & 0.01 & 8.19 & 0.16 & 6.30 & 11.42 \\
\hline $\mathrm{R} 05-17 r$ & 51.02 & 1.03 & 16.49 & 0.00 & 7.83 & 0.19 & 6.08 & 11.41 \\
\hline $\mathrm{R} 05-17 r$ & 50.69 & 0.93 & 15.98 & 0.02 & 8.03 & 0.16 & 7.76 & 11.17 \\
\hline $\mathrm{R} 05-17 \mathrm{r}$ & 50.78 & 0.99 & 16.42 & 0.00 & 8.02 & 0.14 & 6.27 & 11.15 \\
\hline $\mathrm{R} 05-17 \mathrm{r}$ & 50.27 & 0.94 & 16.31 & 0.00 & 7.85 & 0.13 & 6.18 & 10.89 \\
\hline R05-17r & 51.12 & 0.97 & 16.52 & 0.03 & 8.08 & 0.18 & 6.29 & 11.40 \\
\hline $\mathrm{R} 05-17 \mathrm{r}$ & 51.21 & 0.94 & 16.44 & 0.01 & 8.24 & 0.16 & 6.18 & 11.55 \\
\hline $\mathrm{R} 05-17 \mathrm{r}$ & 50.90 & 0.96 & 16.55 & 0.02 & 8.19 & 0.13 & 6.23 & 11.37 \\
\hline $\mathrm{R} 05-17 \mathrm{r}$ & 51.35 & 0.97 & 16.48 & 0.00 & 8.14 & 0.14 & 6.34 & 11.35 \\
\hline $\mathrm{R} 05-17 r$ & 51.10 & 0.97 & 16.54 & 0.03 & 8.17 & 0.16 & 6.24 & 11.43 \\
\hline R05-19r & 51.80 & 1.19 & 16.34 & 0.00 & 8.86 & 0.16 & 4.77 & 9.98 \\
\hline R05-19r & 51.86 & 1.09 & 16.30 & 0.00 & 8.63 & 0.16 & 5.20 & 10.00 \\
\hline R05-19r & 51.94 & 1.16 & 16.16 & 0.00 & 8.92 & 0.14 & 5.22 & 9.90 \\
\hline R05-19r & 51.67 & 1.16 & 16.37 & 0.01 & 8.71 & 0.15 & 5.27 & 9.76 \\
\hline R05-19r & 51.79 & 1.15 & 16.40 & 0.01 & 8.84 & 0.13 & 5.28 & 9.94 \\
\hline R05-19r & 51.76 & 1.12 & 16.20 & 0.02 & 8.92 & 0.18 & 4.96 & 9.88 \\
\hline R05-19r & 51.75 & 1.13 & 16.24 & 0.00 & 8.94 & 0.17 & 5.29 & 10.07 \\
\hline R05-19r & 51.57 & 1.14 & 16.08 & 0.00 & 8.94 & 0.13 & 5.16 & 10.01 \\
\hline R05-19r & 51.62 & 1.17 & 16.18 & 0.00 & 8.99 & 0.15 & 5.16 & 9.94 \\
\hline R05-19r & 52.10 & 1.12 & 16.21 & 0.00 & 8.84 & 0.16 & 5.24 & 9.63 \\
\hline R05-19r & 51.93 & 1.17 & 16.24 & 0.00 & 8.86 & 0.17 & 5.06 & 9.93 \\
\hline R05-19r & 51.93 & 1.18 & 16.00 & 0.01 & 8.75 & 0.14 & 5.20 & 9.92 \\
\hline R05-19r & 51.82 & 1.18 & 16.01 & 0.00 & 8.88 & 0.15 & 5.21 & 9.83 \\
\hline R05-19r & 51.73 & 1.18 & 16.04 & 0.00 & 8.63 & 0.16 & 5.03 & 10.05 \\
\hline R05-19r & 51.65 & 1.16 & 16.19 & 0.00 & 8.77 & 0.14 & 5.20 & 10.02 \\
\hline
\end{tabular}




\begin{tabular}{|c|c|c|c|c|c|c|c|c|}
\hline R05-10r & 50.64 & 0.99 & 16.68 & 0.01 & 7.89 & 0.16 & 6.48 & 11.35 \\
\hline R05-10r & 50.83 & 1.04 & 16.49 & 0.01 & 8.18 & 0.14 & 6.36 & 11.40 \\
\hline R05-10r & 50.54 & 1.00 & 16.35 & 0.01 & 8.25 & 0.16 & 6.01 & 11.28 \\
\hline R05-10r & 50.25 & 1.00 & 16.39 & 0.01 & 8.10 & 0.16 & 6.26 & 11.16 \\
\hline R05-10r & 50.90 & 0.99 & 16.17 & 0.00 & 8.05 & 0.12 & 6.40 & 10.74 \\
\hline R05-10r & 50.91 & 0.97 & 16.48 & 0.00 & 7.91 & 0.15 & 6.42 & 11.18 \\
\hline R05-10r & 50.92 & 1.03 & 16.42 & 0.03 & 8.03 & 0.16 & 6.46 & 11.30 \\
\hline R05-10r & 50.83 & 1.00 & 16.61 & 0.00 & 8.13 & 0.17 & 6.39 & 11.09 \\
\hline R05-10r & 50.84 & 0.95 & 16.47 & 0.02 & 8.23 & 0.16 & 6.16 & 11.27 \\
\hline R05-10r & 50.85 & 1.00 & 16.46 & 0.00 & 8.17 & 0.14 & 6.36 & 11.36 \\
\hline R05-10r & 50.66 & 1.01 & 16.49 & 0.00 & 8.11 & 0.13 & 6.40 & 11.27 \\
\hline R05-10r & 50.67 & 1.03 & 16.53 & 0.05 & 8.02 & 0.15 & 6.38 & 11.33 \\
\hline R05-10r & 50.28 & 0.99 & 16.38 & 0.01 & 8.07 & 0.16 & 6.20 & 11.42 \\
\hline R05-10r & 50.22 & 0.97 & 16.21 & 0.03 & 8.16 & 0.14 & 6.27 & 11.25 \\
\hline R05-10r & 50.40 & 1.01 & 16.24 & 0.02 & 7.92 & 0.17 & 6.31 & 11.39 \\
\hline R05-11 & 51.52 & 1.12 & 16.37 & 0.00 & 8.85 & 0.17 & 5.20 & 9.80 \\
\hline R05-11 & 50.92 & 1.17 & 16.36 & 0.01 & 8.58 & 0.15 & 5.26 & 9.88 \\
\hline R05-11 & 51.18 & 1.12 & 16.45 & 0.00 & 8.88 & 0.18 & 5.28 & 9.87 \\
\hline R05-11 & 51.66 & 1.10 & 16.26 & 0.00 & 8.00 & 0.18 & 5.28 & 9.82 \\
\hline R05-11 & 51.37 & 1.15 & 15.26 & 0.01 & 8.67 & 0.15 & 6.13 & 10.79 \\
\hline R05-11 & 51.45 & 1.12 & 16.52 & 0.00 & 8.42 & 0.19 & 4.90 & 9.87 \\
\hline R05-11 & 51.61 & 1.12 & 16.41 & 0.00 & 8.88 & 0.16 & 5.25 & 9.90 \\
\hline R05-11 & 51.63 & 1.12 & 16.29 & 0.00 & 8.88 & 0.18 & 5.23 & 9.80 \\
\hline R05-11 & 51.83 & 1.14 & 16.37 & 0.01 & 8.71 & 0.14 & 5.25 & 9.94 \\
\hline R05-11 & 51.36 & 1.17 & 16.27 & 0.01 & 8.61 & 0.16 & 5.32 & 9.83 \\
\hline R05-11 & 51.63 & 1.06 & 16.28 & 0.01 & 8.73 & 0.16 & 5.34 & 9.78 \\
\hline R05-11 & 51.17 & 1.18 & 16.28 & 0.01 & 8.91 & 0.14 & 5.13 & 9.26 \\
\hline R05-11 & 51.68 & 1.14 & 16.36 & 0.01 & 8.85 & 0.15 & 4.84 & 9.78 \\
\hline R05-11 & 51.65 & 1.01 & 14.02 & 0.04 & 8.25 & 0.17 & 7.40 & 12.18 \\
\hline R05-11 & 51.72 & 1.15 & 16.30 & 0.00 & 8.93 & 0.16 & 5.26 & 9.87 \\
\hline R05-12 & 50.72 & 0.94 & 16.51 & 0.02 & 8.06 & 0.20 & 6.46 & 11.00 \\
\hline R05-12 & 50.77 & 1.01 & 16.43 & 0.02 & 8.15 & 0.15 & 6.49 & 11.27 \\
\hline R05-12 & 50.86 & 0.98 & 15.91 & 0.02 & 8.13 & 0.14 & 6.24 & 11.37 \\
\hline R05-12 & 50.23 & 0.99 & 16.51 & 0.02 & 8.07 & 0.12 & 6.49 & 11.14 \\
\hline R05-12 & 51.09 & 0.95 & 16.53 & 0.03 & 8.21 & 0.14 & 6.40 & 11.27 \\
\hline R05-12 & 50.94 & 0.97 & 16.52 & 0.00 & 8.17 & 0.16 & 6.36 & 11.09 \\
\hline R05-12 & 50.84 & 0.95 & 16.49 & 0.02 & 8.11 & 0.14 & 6.50 & 11.18 \\
\hline R05-12 & 51.15 & 0.97 & 16.62 & 0.01 & 8.13 & 0.14 & 6.44 & 11.23 \\
\hline R05-12 & 50.99 & 0.93 & 16.42 & 0.01 & 8.11 & 0.15 & 6.47 & 11.22 \\
\hline R05-12 & 50.98 & 1.02 & 16.65 & 0.00 & 8.11 & 0.16 & 6.42 & 11.30 \\
\hline R05-13 & 50.93 & 0.99 & 16.42 & 0.02 & 8.16 & 0.15 & 6.49 & 11.35 \\
\hline R05-13 & 50.71 & 0.99 & 16.42 & 0.02 & 7.99 & 0.16 & 6.47 & 11.02 \\
\hline R05-13 & 51.22 & 0.95 & 16.53 & 0.00 & 8.07 & 0.14 & 6.46 & 11.23 \\
\hline R05-13 & 50.95 & 1.02 & 16.42 & 0.00 & 8.18 & 0.16 & 6.42 & 11.41 \\
\hline R05-13 & 50.89 & 0.98 & 16.48 & 0.00 & 8.15 & 0.19 & 6.47 & 11.18 \\
\hline R05-13 & 51.17 & 0.96 & 16.46 & 0.03 & 8.18 & 0.13 & 6.48 & 11.14 \\
\hline R05-13 & 51.16 & 0.97 & 16.45 & 0.02 & 8.11 & 0.14 & 6.49 & 11.23 \\
\hline
\end{tabular}




$\begin{array}{lllllllll}\text { R05-13 } & 51.08 & 1.00 & 16.37 & 0.00 & 8.14 & 0.14 & 6.45 & 11.24 \\ \text { R05-13 } & 50.56 & 1.02 & 16.29 & 0.00 & 8.09 & 0.13 & 6.37 & 11.28 \\ \text { R05-13 } & 50.97 & 0.98 & 16.53 & 0.01 & 8.05 & 0.18 & 6.40 & 11.28 \\ \text { R05-14 } & 50.79 & 0.95 & 16.31 & 0.00 & 7.91 & 0.17 & 6.51 & 11.22 \\ \text { R05-14 } & 50.65 & 1.03 & 16.36 & 0.02 & 8.13 & 0.16 & 6.50 & 11.12 \\ \text { R05-14 } & 50.87 & 0.95 & 16.48 & 0.01 & 8.19 & 0.16 & 6.43 & 11.30 \\ \text { R05-14 } & 50.95 & 1.00 & 16.23 & 0.00 & 8.21 & 0.13 & 6.11 & 11.31 \\ \text { R05-14 } & 50.62 & 0.98 & 16.37 & 0.03 & 8.09 & 0.15 & 6.41 & 11.30 \\ \text { R05-14 } & 51.04 & 0.97 & 16.56 & 0.00 & 8.31 & 0.15 & 6.48 & 11.36 \\ \text { R05-14 } & 50.50 & 0.99 & 16.49 & 0.01 & 8.08 & 0.15 & 6.37 & 11.26 \\ \text { R05-14 } & 51.03 & 0.93 & 16.42 & 0.00 & 8.17 & 0.13 & 6.04 & 11.33 \\ \text { R05-14 } & 50.95 & 1.05 & 16.34 & 0.01 & 8.05 & 0.13 & 6.40 & 10.60 \\ \text { R05-14 } & 51.09 & 1.02 & 16.40 & 0.00 & 8.12 & 0.13 & 6.42 & 11.25 \\ \text { R05-16 } & 50.99 & 0.98 & 16.45 & 0.02 & 8.23 & 0.13 & 6.53 & 11.29 \\ \text { R05-16 } & 50.85 & 0.97 & 16.37 & 0.04 & 8.12 & 0.17 & 6.51 & 11.35 \\ \text { R05-16 } & 50.94 & 0.96 & 16.56 & 0.00 & 8.06 & 0.14 & 6.46 & 11.33 \\ \text { R05-16 } & 50.84 & 1.03 & 16.41 & 0.02 & 8.15 & 0.18 & 6.54 & 11.20 \\ \text { R05-16 } & 50.95 & 1.01 & 16.48 & 0.03 & 7.89 & 0.19 & 6.50 & 11.10 \\ \text { R05-16 } & 50.93 & 0.97 & 16.40 & 0.02 & 8.00 & 0.16 & 6.39 & 11.35 \\ \text { R05-16 } & 50.84 & 0.97 & 16.51 & 0.00 & 8.19 & 0.14 & 6.44 & 11.38 \\ \text { R05-16 } & 50.53 & 0.96 & 16.46 & 0.03 & 8.16 & 0.12 & 6.41 & 11.21 \\ \text { R05-16 } & 50.97 & 0.96 & 16.01 & 0.01 & 8.03 & 0.14 & 6.57 & 11.02 \\ \text { R05-16 } & 50.72 & 1.01 & 16.25 & 0.00 & 8.25 & 0.14 & 6.48 & 11.21\end{array}$


 \\ Page 97 of 120

\author{
iew
}

Island Arc, For Peer Review

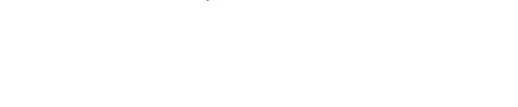

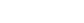

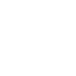

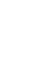

island Arc, For Peer Review 


\begin{tabular}{|c|c|c|c|c|c|c|}
\hline $\mathrm{Na} 2 \mathrm{O}$ & $\mathrm{K} 2 \mathrm{O}$ & P2O5 & $\mathrm{F}$ & $\mathrm{Cl}$ & $\mathrm{SO} 3$ & Total \\
\hline 3.00 & 0.22 & 0.08 & 0.00 & 0.10 & 0.19 & 98.36 \\
\hline 2.77 & 0.20 & 0.13 & 0.00 & 0.09 & 0.19 & 98.02 \\
\hline 2.82 & 0.22 & 0.13 & 0.00 & 0.10 & 0.20 & 97.38 \\
\hline 2.86 & 0.20 & 0.13 & 0.00 & 0.08 & 0.18 & 96.66 \\
\hline 2.83 & 0.22 & 0.14 & 0.08 & 0.09 & 0.18 & 97.87 \\
\hline 2.74 & 0.21 & 0.10 & 0.00 & 0.10 & 0.17 & 97.52 \\
\hline 2.76 & 0.22 & 0.13 & 0.09 & 0.09 & 0.15 & 97.58 \\
\hline 2.85 & 0.21 & 0.09 & 0.02 & 0.08 & 0.21 & 98.03 \\
\hline 2.83 & 0.21 & 0.12 & 0.00 & 0.08 & 0.19 & 97.50 \\
\hline 2.87 & 0.21 & 0.12 & 0.00 & 0.08 & 0.17 & 97.59 \\
\hline 2.81 & 0.22 & 0.07 & 0.00 & 0.10 & 0.20 & 97.30 \\
\hline 2.77 & 0.21 & 0.15 & 0.00 & 0.09 & 0.19 & 97.14 \\
\hline 2.88 & 0.23 & 0.11 & 0.07 & 0.09 & 0.17 & 98.00 \\
\hline 2.39 & 0.20 & 0.10 & 0.00 & 0.08 & 0.17 & 97.82 \\
\hline 2.53 & 0.22 & 0.10 & 0.00 & 0.11 & 0.17 & 97.58 \\
\hline 2.75 & 0.22 & 0.11 & 0.00 & 0.10 & 0.21 & 97.45 \\
\hline 2.87 & 0.23 & 0.14 & 0.00 & 0.09 & 0.21 & 97.88 \\
\hline 2.87 & 0.23 & 0.14 & 0.06 & 0.09 & 0.18 & 97.83 \\
\hline 2.78 & 0.22 & 0.14 & 0.00 & 0.10 & 0.21 & 97.45 \\
\hline 2.77 & 0.20 & 0.13 & 0.00 & 0.07 & 0.19 & 97.73 \\
\hline 2.74 & 0.21 & 0.10 & 0.00 & 0.10 & 0.17 & 97.47 \\
\hline 2.89 & 0.22 & 0.06 & 0.00 & 0.10 & 0.16 & 97.05 \\
\hline 2.86 & 0.21 & 0.14 & 0.00 & 0.10 & 0.21 & 98.05 \\
\hline 2.78 & 0.21 & 0.12 & 0.00 & 0.09 & 0.22 & 97.37 \\
\hline 2.84 & 0.22 & 0.11 & 0.19 & 0.09 & 0.19 & 98.24 \\
\hline 2.89 & 0.21 & 0.12 & 0.00 & 0.10 & 0.20 & 97.72 \\
\hline 2.28 & 0.21 & 0.13 & 0.00 & 0.10 & 0.20 & 97.07 \\
\hline 2.83 & 0.21 & 0.13 & 0.00 & 0.09 & 0.24 & 97.74 \\
\hline 2.81 & 0.24 & 0.10 & 0.05 & 0.11 & 0.18 & 98.02 \\
\hline 2.82 & 0.22 & 0.12 & 0.00 & 0.10 & 0.20 & 98.19 \\
\hline 2.84 & 0.20 & 0.08 & 0.00 & 0.08 & 0.19 & 97.54 \\
\hline 2.76 & 0.21 & 0.10 & 0.00 & 0.10 & 0.19 & 98.13 \\
\hline 2.89 & 0.23 & 0.14 & 0.08 & 0.10 & 0.18 & 97.62 \\
\hline 2.77 & 0.23 & 0.10 & 0.00 & 0.10 & 0.21 & 97.71 \\
\hline 2.83 & 0.21 & 0.09 & 0.00 & 0.09 & 0.21 & 97.50 \\
\hline 2.45 & 0.21 & 0.08 & 0.06 & 0.08 & 0.19 & 97.04 \\
\hline 2.75 & 0.21 & 0.11 & 0.09 & 0.09 & 0.18 & 96.96 \\
\hline 2.71 & 0.21 & 0.07 & 0.04 & 0.08 & 0.22 & 97.97 \\
\hline 2.86 & 0.22 & 0.10 & 0.05 & 0.09 & 0.18 & 97.36 \\
\hline 2.76 & 0.22 & 0.13 & 0.00 & 0.10 & 0.21 & 97.62 \\
\hline 2.86 & 0.22 & 0.17 & 0.00 & 0.09 & 0.19 & 97.65 \\
\hline 2.72 & 0.22 & 0.11 & 0.00 & 0.09 & 0.20 & 97.68 \\
\hline 2.75 & 0.23 & 0.11 & 0.00 & 0.08 & 0.20 & 97.61 \\
\hline 2.54 & 0.20 & 0.11 & 0.17 & 0.08 & 0.19 & 98.02 \\
\hline 2.81 & 0.20 & 0.14 & 0.02 & 0.08 & 0.20 & 98.05 \\
\hline 2.79 & 0.22 & 0.08 & 0.00 & 0.08 & 0.18 & 97.48 \\
\hline
\end{tabular}




\begin{tabular}{|c|c|c|c|c|c|c|}
\hline 2.33 & 0.22 & 0.07 & 0.00 & 0.08 & 0.21 & 96.24 \\
\hline 2.89 & 0.23 & 0.09 & 0.00 & 0.08 & 0.20 & 97.10 \\
\hline 2.77 & 0.23 & 0.16 & 0.00 & 0.10 & 0.18 & 97.33 \\
\hline 2.82 & 0.22 & 0.13 & 0.01 & 0.09 & 0.18 & 97.70 \\
\hline 2.86 & 0.23 & 0.12 & 0.00 & 0.09 & 0.19 & 97.20 \\
\hline 2.89 & 0.21 & 0.12 & 0.00 & 0.09 & 0.18 & 97.54 \\
\hline 2.82 & 0.22 & 0.11 & 0.00 & 0.09 & 0.17 & 97.51 \\
\hline 2.82 & 0.23 & 0.08 & 0.05 & 0.09 & 0.19 & 97.41 \\
\hline 2.84 & 0.22 & 0.14 & 0.00 & 0.07 & 0.20 & 97.31 \\
\hline 2.69 & 0.22 & 0.13 & 0.00 & 0.10 & 0.19 & 97.80 \\
\hline 2.83 & 0.23 & 0.12 & 0.25 & 0.09 & 0.20 & 97.14 \\
\hline 2.88 & 0.23 & 0.12 & 0.00 & 0.10 & 0.18 & 97.61 \\
\hline 2.89 & 0.21 & 0.12 & 0.00 & 0.09 & 0.20 & 97.99 \\
\hline 2.74 & 0.22 & 0.13 & 0.01 & 0.09 & 0.16 & 97.83 \\
\hline 2.57 & 0.21 & 0.11 & 0.00 & 0.09 & 0.21 & 97.75 \\
\hline 2.88 & 0.22 & 0.12 & 0.00 & 0.09 & 0.22 & 98.01 \\
\hline 2.85 & 0.20 & 0.09 & 0.06 & 0.10 & 0.20 & 97.04 \\
\hline 2.81 & 0.21 & 0.08 & 0.08 & 0.09 & 0.18 & 97.19 \\
\hline 2.80 & 0.21 & 0.12 & 0.02 & 0.08 & 0.18 & 96.28 \\
\hline 2.46 & 0.23 & 0.07 & 0.08 & 0.08 & 0.16 & 96.85 \\
\hline 2.65 & 0.21 & 0.11 & 0.18 & 0.09 & 0.18 & 97.56 \\
\hline 2.74 & 0.20 & 0.12 & 0.00 & 0.08 & 0.19 & 97.89 \\
\hline 2.79 & 0.21 & 0.14 & 0.00 & 0.10 & 0.17 & 97.65 \\
\hline 2.95 & 0.23 & 0.11 & 0.00 & 0.08 & 0.19 & 97.60 \\
\hline 2.69 & 0.21 & 0.12 & 0.01 & 0.09 & 0.19 & 98.04 \\
\hline 2.83 & 0.22 & 0.07 & 0.00 & 0.10 & 0.18 & 97.15 \\
\hline 2.78 & 0.21 & 0.11 & 0.00 & 0.09 & 0.16 & 95.90 \\
\hline 2.83 & 0.22 & 0.08 & 0.00 & 0.09 & 0.22 & 98.02 \\
\hline 2.75 & 0.22 & 0.10 & 0.00 & 0.11 & 0.22 & 98.09 \\
\hline 2.82 & 0.22 & 0.06 & 0.07 & 0.07 & 0.23 & 97.77 \\
\hline 2.75 & 0.21 & 0.11 & 0.00 & 0.09 & 0.18 & 98.09 \\
\hline 2.85 & 0.21 & 0.08 & 0.00 & 0.09 & 0.19 & 98.03 \\
\hline 3.13 & 0.26 & 0.11 & 0.09 & 0.13 & 0.17 & 96.92 \\
\hline 3.11 & 0.25 & 0.09 & 0.00 & 0.11 & 0.20 & 96.98 \\
\hline 3.19 & 0.26 & 0.11 & 0.00 & 0.15 & 0.24 & 97.35 \\
\hline 3.17 & 0.24 & 0.08 & 0.09 & 0.13 & 0.17 & 96.92 \\
\hline 3.16 & 0.26 & 0.13 & 0.07 & 0.14 & 0.21 & 97.43 \\
\hline 3.00 & 0.24 & 0.13 & 0.03 & 0.12 & 0.23 & 96.75 \\
\hline 3.16 & 0.25 & 0.10 & 0.03 & 0.12 & 0.24 & 97.44 \\
\hline 3.06 & 0.25 & 0.15 & 0.00 & 0.11 & 0.21 & 96.80 \\
\hline 3.06 & 0.27 & 0.09 & 0.03 & 0.11 & 0.17 & 96.90 \\
\hline 3.07 & 0.26 & 0.12 & 0.00 & 0.13 & 0.22 & 97.08 \\
\hline 3.10 & 0.27 & 0.11 & 0.05 & 0.12 & 0.19 & 97.17 \\
\hline 3.19 & 0.26 & 0.14 & 0.00 & 0.13 & 0.22 & 97.04 \\
\hline 3.13 & 0.26 & 0.12 & 0.00 & 0.13 & 0.20 & 96.90 \\
\hline 3.07 & 0.27 & 0.11 & 0.08 & 0.13 & 0.21 & 96.62 \\
\hline 3.22 & 0.26 & 0.16 & 0.00 & 0.12 & 0.19 & 97.05 \\
\hline
\end{tabular}




\begin{tabular}{|c|c|c|c|c|c|c|}
\hline 2.80 & 0.21 & 0.10 & 0.08 & 0.08 & 0.19 & 97.59 \\
\hline 2.79 & 0.21 & 0.11 & 0.07 & 0.08 & 0.17 & 97.82 \\
\hline 2.46 & 0.21 & 0.11 & 0.08 & 0.09 & 0.22 & 96.71 \\
\hline 2.62 & 0.26 & 0.12 & 0.05 & 0.12 & 0.18 & 96.62 \\
\hline 2.85 & 0.21 & 0.09 & 0.05 & 0.09 & 0.18 & 96.81 \\
\hline 2.80 & 0.22 & 0.11 & 0.00 & 0.08 & 0.20 & 97.43 \\
\hline 2.66 & 0.21 & 0.12 & 0.00 & 0.09 & 0.18 & 97.59 \\
\hline 2.92 & 0.23 & 0.12 & 0.09 & 0.09 & 0.19 & 97.79 \\
\hline 2.81 & 0.24 & 0.13 & 0.05 & 0.08 & 0.18 & 97.55 \\
\hline 2.89 & 0.20 & 0.07 & 0.00 & 0.07 & 0.19 & 97.75 \\
\hline 2.82 & 0.21 & 0.15 & 0.00 & 0.09 & 0.23 & 97.54 \\
\hline 2.81 & 0.22 & 0.16 & 0.00 & 0.09 & 0.18 & 97.61 \\
\hline 2.78 & 0.21 & 0.14 & 0.00 & 0.08 & 0.21 & 96.91 \\
\hline 2.76 & 0.21 & 0.12 & 0.03 & 0.08 & 0.19 & 96.60 \\
\hline 2.83 & 0.21 & 0.14 & 0.00 & 0.08 & 0.22 & 96.92 \\
\hline 3.32 & 0.26 & & 0.02 & 0.16 & 0.20 & 97.05 \\
\hline 3.38 & 0.25 & & 0.08 & 0.13 & 0.20 & 96.35 \\
\hline 3.08 & 0.27 & & 0.00 & 0.13 & 0.16 & 96.60 \\
\hline 3.34 & 0.25 & & 0.04 & 0.13 & 0.15 & 96.18 \\
\hline 3.00 & 0.23 & & 0.00 & 0.13 & 0.20 & 97.15 \\
\hline 3.22 & 0.23 & & 0.00 & 0.15 & 0.14 & 96.25 \\
\hline 3.33 & 0.25 & & 0.11 & 0.16 & 0.18 & 97.35 \\
\hline 3.30 & 0.26 & & 0.09 & 0.14 & 0.16 & 97.06 \\
\hline 3.40 & 0.25 & & 0.00 & 0.14 & 0.20 & 97.38 \\
\hline 3.16 & 0.23 & & 0.00 & 0.14 & 0.19 & 96.47 \\
\hline 3.23 & 0.25 & & 0.15 & 0.14 & 0.19 & 96.93 \\
\hline 3.07 & 0.29 & & 0.05 & 0.17 & 0.17 & 95.89 \\
\hline 3.06 & 0.27 & & 0.00 & 0.13 & 0.19 & 96.48 \\
\hline 2.38 & 0.23 & & 0.00 & 0.11 & 0.18 & 97.61 \\
\hline 3.23 & 0.26 & & 0.05 & 0.14 & 0.16 & 97.24 \\
\hline 3.02 & 0.22 & & 0.00 & 0.09 & 0.17 & 97.48 \\
\hline 2.77 & 0.22 & & 0.00 & 0.10 & 0.20 & 97.62 \\
\hline 2.95 & 0.22 & & 0.13 & 0.10 & 0.21 & 97.20 \\
\hline 2.90 & 0.20 & & 0.00 & 0.11 & 0.20 & 97.02 \\
\hline 2.90 & 0.21 & & 0.00 & 0.10 & 0.17 & 98.01 \\
\hline 2.94 & 0.21 & & 0.00 & 0.10 & 0.20 & 97.68 \\
\hline 2.91 & 0.21 & & 0.00 & 0.09 & 0.20 & 97.65 \\
\hline 2.99 & 0.21 & & 0.14 & 0.09 & 0.20 & 98.32 \\
\hline 2.82 & 0.21 & & 0.00 & 0.10 & 0.24 & 97.67 \\
\hline 2.98 & 0.22 & & 0.09 & 0.10 & 0.19 & 98.22 \\
\hline 2.94 & 0.21 & & 0.00 & 0.09 & 0.19 & 97.92 \\
\hline 2.84 & 0.21 & & 0.00 & 0.09 & 0.18 & 97.09 \\
\hline 2.95 & 0.20 & & 0.00 & 0.09 & 0.18 & 98.02 \\
\hline 2.93 & 0.20 & & 0.00 & 0.08 & 0.21 & 97.99 \\
\hline 2.99 & 0.21 & & 0.03 & 0.10 & 0.20 & 97.87 \\
\hline 3.01 & 0.22 & & 0.00 & 0.09 & 0.19 & 98.07 \\
\hline 2.83 & 0.21 & & 0.00 & 0.09 & 0.20 & 97.93 \\
\hline
\end{tabular}




$\begin{array}{llllll}2.94 & 0.22 & 0.03 & 0.09 & 0.23 & 97.93 \\ 2.89 & 0.20 & 0.00 & 0.10 & 0.23 & 97.18 \\ 2.98 & 0.21 & 0.00 & 0.09 & 0.18 & 97.85 \\ 2.61 & 0.23 & 0.00 & 0.10 & 0.17 & 96.97 \\ 2.98 & 0.22 & 0.08 & 0.08 & 0.17 & 97.50 \\ 2.90 & 0.21 & 0.07 & 0.09 & 0.18 & 97.82 \\ 2.94 & 0.22 & 0.00 & 0.07 & 0.17 & 97.36 \\ 2.95 & 0.21 & 0.01 & 0.10 & 0.16 & 97.39 \\ 2.90 & 0.21 & 0.00 & 0.10 & 0.21 & 98.31 \\ 2.99 & 0.21 & 0.06 & 0.10 & 0.22 & 97.37 \\ 3.01 & 0.21 & 0.06 & 0.10 & 0.15 & 97.64 \\ 2.87 & 0.21 & 0.00 & 0.09 & 0.20 & 96.90 \\ 2.96 & 0.21 & 0.00 & 0.09 & 0.21 & 97.91 \\ 2.89 & 0.19 & 0.06 & 0.10 & 0.17 & 98.04 \\ 2.89 & 0.22 & 0.03 & 0.09 & 0.16 & 97.74 \\ 2.87 & 0.23 & 0.00 & 0.09 & 0.18 & 97.83 \\ 2.93 & 0.21 & 0.09 & 0.08 & 0.17 & 97.78 \\ 2.95 & 0.20 & 0.00 & 0.09 & 0.18 & 97.56 \\ 3.00 & 0.22 & 0.06 & 0.10 & 0.18 & 97.76 \\ 2.86 & 0.23 & 0.00 & 0.10 & 0.18 & 97.86 \\ 2.93 & 0.20 & 0.00 & 0.10 & 0.15 & 97.30 \\ 2.93 & 0.22 & 0.00 & 0.10 & 0.17 & 97.14 \\ 2.79 & 0.23 & 0.00 & 0.09 & 0.22 & 97.39\end{array}$




\begin{tabular}{|c|c|c|c|c|c|c|c|c|}
\hline $4 / 23 / 2014$ & $\mathrm{SiO} 2$ & TiO2 & $\mathrm{Al} 2 \mathrm{O} 3$ & $\mathrm{Cr} 2 \mathrm{O} 3$ & $\mathrm{FeO}$ & $\mathrm{MnO}$ & $\mathrm{MgO}$ & $\mathrm{CaO}$ \\
\hline R06-11r & 51.03 & 1.16 & 16.44 & 0.01 & 8.59 & 0.19 & 5.28 & 10.08 \\
\hline R06-11r & 51.55 & 1.13 & 16.46 & 0.02 & 8.73 & 0.17 & 5.07 & 10.01 \\
\hline R06-11r & 51.77 & 1.18 & 16.49 & 0.00 & 8.80 & 0.17 & 5.30 & 10.13 \\
\hline R06-11r & 51.51 & 1.17 & 16.41 & 0.00 & 8.64 & 0.19 & 5.24 & 9.84 \\
\hline R06-11r & 51.64 & 1.12 & 16.33 & 0.00 & 8.83 & 0.15 & 5.36 & 10.01 \\
\hline R06-11r & 51.55 & 1.14 & 16.32 & 0.00 & 8.54 & 0.18 & 5.17 & 10.19 \\
\hline R06-11r & 51.75 & 1.14 & 16.47 & 0.00 & 8.77 & 0.15 & 5.25 & 10.08 \\
\hline R06-11r & 51.66 & 1.13 & 16.50 & 0.00 & 8.76 & 0.15 & 5.27 & 10.10 \\
\hline R06-11r & 51.60 & 1.13 & 16.04 & 0.01 & 8.69 & 0.14 & 5.28 & 9.94 \\
\hline R06-11r & 51.67 & 1.07 & 16.51 & 0.01 & 8.78 & 0.15 & 5.32 & 10.00 \\
\hline R06-11r & 51.68 & 1.07 & 14.49 & 0.03 & 8.49 & 0.19 & 7.23 & 11.77 \\
\hline R06-11r & 51.78 & 1.12 & 16.30 & 0.00 & 8.68 & 0.15 & 5.25 & 10.01 \\
\hline R06-11r & 51.74 & 1.07 & 16.24 & 0.03 & 8.87 & 0.15 & 5.32 & 9.98 \\
\hline R06-11r & 51.82 & 1.14 & 16.49 & 0.02 & 8.91 & 0.13 & 5.33 & 9.73 \\
\hline R06-11r & 51.88 & 1.14 & 16.33 & 0.02 & 8.60 & 0.15 & 5.34 & 10.12 \\
\hline R06-12r & 50.97 & 1.04 & 16.58 & 0.01 & 8.48 & 0.13 & 6.34 & 11.30 \\
\hline R06-12r & 50.99 & 1.02 & 16.51 & 0.01 & 8.53 & 0.17 & 6.38 & 11.25 \\
\hline R06-12r & 51.18 & 1.01 & 16.62 & 0.00 & 8.48 & 0.15 & 6.32 & 11.00 \\
\hline R06-12r & 51.06 & 1.05 & 16.52 & 0.01 & 8.38 & 0.14 & 6.28 & 11.33 \\
\hline R06-12r & 50.92 & 1.00 & 16.52 & 0.01 & 8.33 & 0.15 & 6.46 & 11.21 \\
\hline$R 06-12 r$ & 50.66 & 1.06 & 16.47 & 0.00 & 8.29 & 0.18 & 6.27 & 11.37 \\
\hline R06-12r & 50.99 & 1.07 & 16.68 & 0.01 & 8.41 & 0.16 & 6.25 & 11.03 \\
\hline$R 06-12 r$ & 51.07 & 1.02 & 16.53 & 0.00 & 8.25 & 0.16 & 6.36 & 11.37 \\
\hline R06-12r & 51.16 & 1.05 & 16.46 & 0.02 & 8.36 & 0.15 & 6.31 & 11.28 \\
\hline R06-12r & 51.08 & 1.05 & 15.86 & 0.02 & 8.32 & 0.13 & 6.31 & 11.42 \\
\hline R06-12r & 51.12 & 0.98 & 16.54 & 0.01 & 8.52 & 0.17 & 5.93 & 11.33 \\
\hline R06-12r & 50.62 & 1.03 & 16.55 & 0.01 & 8.20 & 0.16 & 6.15 & 11.15 \\
\hline R06-12r & 50.90 & 1.04 & 16.59 & 0.01 & 8.28 & 0.11 & 6.23 & 11.29 \\
\hline$R 06-12 r$ & 50.39 & 1.07 & 16.55 & 0.00 & 8.26 & 0.17 & 6.22 & 11.31 \\
\hline $\mathrm{R} 06-12 \mathrm{r}$ & 50.34 & 0.99 & 16.56 & 0.00 & 8.39 & 0.14 & 6.24 & 11.34 \\
\hline R06-13r & 51.09 & 1.04 & 16.63 & 0.01 & 8.36 & 0.15 & 6.31 & 11.43 \\
\hline R06-13r & 51.05 & 0.99 & 16.16 & 0.01 & 8.47 & 0.15 & 6.38 & 11.34 \\
\hline R06-13r & 51.07 & 1.00 & 16.60 & 0.00 & 8.46 & 0.16 & 6.36 & 11.38 \\
\hline R06-13r & 50.84 & 0.96 & 16.65 & 0.01 & 8.47 & 0.14 & 6.41 & 11.41 \\
\hline R06-13r & 50.83 & 1.01 & 16.25 & 0.02 & 8.14 & 0.16 & 6.35 & 11.36 \\
\hline R06-13r & 51.04 & 1.05 & 16.56 & 0.00 & 8.38 & 0.17 & 6.29 & 11.36 \\
\hline R06-13r & 50.86 & 1.00 & 16.49 & 0.00 & 8.21 & 0.16 & 6.28 & 11.47 \\
\hline R06-13r & 50.89 & 1.00 & 16.50 & 0.00 & 8.27 & 0.17 & 6.35 & 11.48 \\
\hline R06-13r & 51.02 & 1.05 & 16.55 & 0.01 & 8.52 & 0.14 & 6.38 & 11.32 \\
\hline R06-13r & 50.56 & 0.99 & 16.16 & 0.00 & 8.47 & 0.15 & 6.24 & 11.40 \\
\hline R06-13r & 50.87 & 1.04 & 16.34 & 0.01 & 8.20 & 0.13 & 6.35 & 11.40 \\
\hline R06-13r & 50.95 & 1.04 & 16.49 & 0.01 & 8.46 & 0.14 & 6.01 & 11.44 \\
\hline R06-13r & 50.91 & 1.02 & 16.22 & 0.00 & 8.39 & 0.17 & 6.37 & 11.27 \\
\hline R06-13r & 50.99 & 1.05 & 16.53 & 0.00 & 8.37 & 0.18 & 6.38 & 11.34 \\
\hline R06-13r & 50.90 & 1.05 & 16.42 & 0.01 & 8.41 & 0.17 & 6.33 & 11.13 \\
\hline R06-14r & 51.37 & 1.10 & 16.64 & 0.01 & 8.69 & 0.15 & 4.89 & 9.85 \\
\hline
\end{tabular}




\begin{tabular}{|c|c|c|c|c|c|c|c|c|}
\hline R06-14r & 51.73 & 1.17 & 16.63 & 0.00 & 8.75 & 0.17 & 4.89 & 9.75 \\
\hline R06-14r & 51.64 & 1.12 & 16.18 & 0.01 & 8.83 & 0.15 & 4.95 & 9.51 \\
\hline R06-14r & 51.60 & 1.17 & 16.37 & 0.00 & 8.72 & 0.16 & 4.89 & 9.79 \\
\hline R06-14r & 51.58 & 1.20 & 16.61 & 0.01 & 8.74 & 0.18 & 4.91 & 9.89 \\
\hline R06-14r & 49.93 & 1.10 & 15.85 & 0.00 & 8.53 & 0.19 & 5.12 & 10.00 \\
\hline $\mathrm{R} 06-14 \mathrm{r}$ & 51.68 & 1.20 & 16.24 & 0.01 & 8.69 & 0.14 & 4.98 & 10.02 \\
\hline R06-14r & 51.75 & 1.16 & 16.33 & 0.00 & 8.59 & 0.19 & 5.10 & 10.24 \\
\hline R06-14r & 51.64 & 1.10 & 16.65 & 0.00 & 8.66 & 0.15 & 4.90 & 9.92 \\
\hline R06-14r & 51.56 & 1.13 & 16.40 & 0.02 & 8.59 & 0.17 & 5.04 & 10.17 \\
\hline R06-14r & 51.68 & 1.10 & 16.68 & 0.00 & 8.78 & 0.15 & 4.85 & 10.04 \\
\hline R06-14r & 51.54 & 1.15 & 16.68 & 0.00 & 8.73 & 0.15 & 4.89 & 9.94 \\
\hline R06-14r & 51.70 & 1.20 & 16.25 & 0.03 & 8.52 & 0.16 & 4.91 & 9.82 \\
\hline R06-15r & 51.59 & 1.15 & 16.12 & 0.00 & 8.94 & 0.18 & 5.44 & 9.93 \\
\hline R06-15r & 51.77 & 1.14 & 16.26 & 0.00 & 9.11 & 0.16 & 5.34 & 9.94 \\
\hline R06-15r & 52.04 & 1.10 & 16.35 & 0.00 & 8.89 & 0.15 & 5.37 & 9.94 \\
\hline R06-15r & 51.81 & 1.14 & 16.46 & 0.00 & 8.82 & 0.14 & 5.29 & 9.93 \\
\hline R06-15r & 51.66 & 1.10 & 16.35 & 0.01 & 8.95 & 0.16 & 5.27 & 10.02 \\
\hline R06-15r & 51.84 & 1.16 & 16.22 & 0.00 & 8.92 & 0.15 & 5.40 & 10.02 \\
\hline R06-15r & 50.98 & 1.14 & 16.27 & 0.01 & 9.05 & 0.12 & 5.09 & 9.16 \\
\hline R06-15r & 51.85 & 1.16 & 16.36 & 0.02 & 8.91 & 0.15 & 5.40 & 9.85 \\
\hline R06-15r & 51.97 & 1.08 & 16.45 & 0.02 & 9.00 & 0.18 & 5.03 & 10.03 \\
\hline R06-15r & 51.71 & 1.15 & 16.51 & 0.00 & 8.81 & 0.18 & 5.26 & 10.03 \\
\hline R06-15r & 51.41 & 1.09 & 15.91 & 0.01 & 9.13 & 0.16 & 6.77 & 9.40 \\
\hline R06-15r & 51.89 & 1.07 & 16.15 & 0.01 & 8.97 & 0.14 & 5.35 & 9.66 \\
\hline R06-15r & 51.73 & 1.15 & 16.44 & 0.01 & 8.55 & 0.16 & 5.37 & 9.98 \\
\hline R06-15r & 51.87 & 1.18 & 16.31 & 0.01 & 8.82 & 0.17 & 5.31 & 10.05 \\
\hline R06-15r & 51.70 & 1.15 & 16.40 & 0.00 & 8.96 & 0.12 & 5.31 & 9.84 \\
\hline R06-16r & 51.81 & 1.11 & 16.36 & 0.02 & 8.80 & 0.16 & 5.34 & 10.05 \\
\hline R06-16r & 51.74 & 1.07 & 16.34 & 0.03 & 8.85 & 0.16 & 5.41 & 10.15 \\
\hline R06-16r & 51.85 & 1.13 & 16.38 & 0.02 & 8.70 & 0.14 & 5.18 & 10.09 \\
\hline R06-16r & 51.70 & 1.14 & 16.46 & 0.01 & 8.90 & 0.16 & 5.39 & 10.06 \\
\hline R06-16r & 50.65 & 1.16 & 16.22 & 0.00 & 9.01 & 0.19 & 5.49 & 10.12 \\
\hline R06-16r & 51.30 & 1.10 & 16.17 & 0.00 & 8.89 & 0.17 & 5.43 & 10.01 \\
\hline R06-16r & 51.66 & 1.12 & 16.41 & 0.00 & 8.88 & 0.19 & 5.16 & 10.03 \\
\hline R06-16r & 51.21 & 1.17 & 16.06 & 0.00 & 8.83 & 0.16 & 5.37 & 10.06 \\
\hline R06-16r & 51.78 & 1.10 & 16.38 & 0.01 & 8.87 & 0.16 & 5.47 & 9.66 \\
\hline R06-16r & 51.80 & 1.14 & 16.41 & 0.03 & 8.80 & 0.16 & 5.33 & 10.14 \\
\hline R06-16r & 51.65 & 1.08 & 16.43 & 0.00 & 8.90 & 0.15 & 5.49 & 10.04 \\
\hline R06-16r & 50.89 & 1.13 & 16.38 & 0.00 & 8.80 & 0.18 & 5.44 & 10.10 \\
\hline R06-16r & 51.53 & 1.09 & 16.21 & 0.00 & 8.83 & 0.15 & 5.33 & 10.06 \\
\hline R06-16r & 51.87 & 1.18 & 16.46 & 0.00 & 8.86 & 0.16 & 5.40 & 10.07 \\
\hline R06-11 & 51.52 & 1.09 & 16.50 & 0.02 & 8.79 & 0.16 & 5.33 & 9.67 \\
\hline R06-11 & 51.56 & 1.12 & 16.46 & 0.01 & 8.76 & 0.18 & 5.32 & 9.99 \\
\hline R06-11 & 51.78 & 1.12 & 16.52 & 0.01 & 8.80 & 0.14 & 5.30 & 9.99 \\
\hline R06-11 & 51.50 & 1.14 & 16.42 & 0.02 & 8.69 & 0.18 & 5.49 & 9.91 \\
\hline R06-11 & 51.63 & 1.14 & 16.48 & 0.01 & 8.58 & 0.16 & 5.40 & 10.00 \\
\hline R06-11 & 51.74 & 1.15 & 16.43 & 0.01 & 8.60 & 0.17 & 5.37 & 10.00 \\
\hline
\end{tabular}




\begin{tabular}{|c|c|c|c|c|c|c|c|c|}
\hline R06-11 & 50.81 & 1.13 & 16.30 & 0.00 & 8.76 & 0.14 & 5.33 & 10.06 \\
\hline R06-11 & 51.59 & 1.08 & 16.49 & 0.00 & 8.82 & 0.16 & 5.05 & 10.06 \\
\hline R06-11 & 51.45 & 1.16 & 16.46 & 0.00 & 8.74 & 0.17 & 5.42 & 10.01 \\
\hline R06-11 & 51.70 & 1.19 & 16.44 & 0.00 & 8.44 & 0.13 & 5.32 & \\
\hline R06-12 & 51.24 & 1.09 & 16.49 & 0.01 & 8.66 & 0.18 & 5.21 & 9.4 \\
\hline R06-12 & 51.37 & 1.10 & 16.81 & 0.02 & 8.72 & 0.16 & 4.79 & 9. \\
\hline R06-12 & 51.53 & 1.12 & 16.85 & 0.00 & 8.67 & 0.16 & 4.66 & \\
\hline R06-12 & 51.47 & 1.19 & 16.71 & 0.02 & 8.86 & 0.15 & 4.86 & 9.7 \\
\hline R06-12 & 51.32 & 1.12 & 16.07 & 0.01 & 8.65 & 0.18 & 5.33 & 10. \\
\hline R06-12 & 51.65 & 1.15 & 16.69 & 0.00 & 8.72 & 0.18 & 4.93 & 9.7 \\
\hline R06-12 & 51.44 & 1.15 & 16.33 & 0.02 & 8.77 & 0.14 & 5.06 & 10.0 \\
\hline R06-12 & 51.13 & 1.16 & 16.42 & 0.00 & 8.38 & 0.15 & 5.16 & \\
\hline R06-12 & 51.53 & 1.15 & 16.48 & 0.01 & 8.45 & 0.14 & 4.98 & 9.9 \\
\hline R06-12 & 51.64 & 1.14 & 16.43 & 0.00 & 8.62 & 0.14 & 5.09 & 9.9 \\
\hline R06-13 & 50.78 & 0.97 & 16.55 & 0.02 & 8.35 & 0.14 & 6.45 & 11. \\
\hline R06-13 & 50.78 & 1.10 & 16.17 & 0.01 & 8.21 & 0.14 & 6.39 & 11.32 \\
\hline R06-13 & 50.98 & 1.04 & 16.49 & 0.03 & 8.39 & 0.16 & 6.43 & 11. \\
\hline R06-13 & 50.98 & 1.03 & 16.55 & 0.01 & 8.50 & 0.18 & 6.15 & 11.02 \\
\hline R06-13 & 51.03 & 1.04 & 16.25 & 0.01 & 8.29 & 0.14 & 6.15 & 11. \\
\hline R06-13 & 50.73 & 1.00 & 16.36 & 0.01 & 8.37 & 0.13 & 6.46 & 11.2 \\
\hline R06-13 & 50.48 & 0.99 & 16.48 & 0.01 & 8.53 & 0.18 & 6.45 & 11.4 \\
\hline R06-13 & 51.02 & 1.00 & 16.58 & 0.00 & 8.28 & 0.15 & 6.50 & 11.2 \\
\hline R06-13 & 50.94 & 1.01 & 16.37 & 0.00 & 8.44 & 0.15 & 6.46 & 11.3 \\
\hline R06-14 & 51.62 & 1.23 & 16.64 & 0.01 & 8.66 & 0.17 & 4.95 & 9.87 \\
\hline R06-14 & 51.47 & 1.12 & 16.62 & 0.01 & 8.84 & 0.16 & 4.97 & 9. \\
\hline R06-14 & 51.41 & 1.11 & 16.63 & 0.00 & 8.84 & 0.18 & 4.99 & 9. \\
\hline R06-14 & 51.68 & 1.13 & 16.39 & 0.00 & 8.71 & 0.16 & 5.08 & 10.0 \\
\hline R06-14 & 51.51 & 1.14 & 16.53 & 0.01 & 8.73 & 0.15 & 4.95 & 10. \\
\hline R06-14 & 51.68 & 1.12 & 16.45 & 0.00 & 8.52 & 0.12 & 4.88 & 9.9 \\
\hline R06-14 & 51.39 & 1.09 & 16.50 & 0.00 & 8.48 & 0.17 & 4.81 & 9.6 \\
\hline R06-14 & 51.08 & 1.19 & 16.48 & 0.00 & 8.63 & 0.12 & 4.96 & 9.7 \\
\hline R06-14 & 51.60 & 1.13 & 16.62 & 0.00 & 8.82 & 0.15 & 5.00 & \\
\hline R06-14 & 51.64 & 1.16 & 16.47 & 0.00 & 8.76 & 0.18 & 4.90 & 9. \\
\hline R06-15 & 51.94 & 1.23 & 16.27 & 0.02 & 8.90 & 0.15 & 5.48 & 9. \\
\hline R06-15 & 51.56 & 1.15 & 16.16 & 0.00 & 8.81 & 0.18 & 5.45 & 9.8 \\
\hline R06-15 & 51.83 & 1.22 & 16.18 & 0.02 & 8.94 & 0.18 & 5.49 & 9.9 \\
\hline R06-15 & 51.78 & 1.16 & 16.25 & 0.01 & 8.75 & 0.13 & 5.37 & 10.0 \\
\hline R06-15 & 51.79 & 1.13 & 16.27 & 0.02 & 8.68 & 0.16 & 5.32 & 9.9 \\
\hline R06-15 & 51.74 & 1.16 & 15.86 & 0.00 & 8.84 & 0.16 & 5.35 & 9.9 \\
\hline R06-15 & 51.99 & 1.13 & 16.33 & 0.00 & 8.88 & 0.12 & 5.42 & \\
\hline R06-15 & 51.67 & 1.13 & 16.02 & 0.01 & 8.84 & 0.18 & 5.35 & \\
\hline R06-15 & 51.65 & 1.15 & 15.99 & 0.00 & 8.93 & 0.21 & 5.19 & \\
\hline R06-15 & 50.75 & 1.03 & 15.11 & 0.00 & 9.77 & 0.15 & 8.85 & \\
\hline
\end{tabular}




\begin{tabular}{|c|c|c|c|c|c|c|}
\hline $\mathrm{Na} 2 \mathrm{O}$ & $\mathrm{K} 2 \mathrm{O}$ & P2O5 & $\mathrm{F}$ & $\mathrm{Cl}$ & SO3 & Total \\
\hline 3.18 & 0.27 & 0.17 & 0.00 & 0.16 & 0.22 & 96.72 \\
\hline 3.21 & 0.25 & 0.15 & 0.00 & 0.14 & 0.20 & 97.06 \\
\hline 3.06 & 0.24 & 0.16 & 0.00 & 0.14 & 0.20 & 97.62 \\
\hline 3.18 & 0.25 & 0.15 & 0.02 & 0.13 & 0.19 & 96.87 \\
\hline 3.22 & 0.26 & 0.14 & 0.00 & 0.15 & 0.20 & 97.37 \\
\hline 3.15 & 0.26 & 0.15 & 0.00 & 0.14 & 0.20 & 96.95 \\
\hline 3.02 & 0.25 & 0.14 & 0.07 & 0.12 & 0.18 & 97.34 \\
\hline 3.01 & 0.25 & 0.13 & 0.01 & 0.12 & 0.20 & 97.27 \\
\hline 3.26 & 0.25 & 0.10 & 0.05 & 0.13 & 0.17 & 96.73 \\
\hline 3.31 & 0.25 & 0.15 & 0.00 & 0.16 & 0.19 & 97.55 \\
\hline 2.69 & 0.22 & 0.09 & 0.01 & 0.11 & 0.17 & 98.22 \\
\hline 3.09 & 0.27 & 0.12 & 0.06 & 0.15 & 0.21 & 97.14 \\
\hline 3.15 & 0.26 & 0.11 & 0.22 & 0.15 & 0.18 & 97.34 \\
\hline 3.15 & 0.24 & 0.07 & 0.04 & 0.14 & 0.21 & 97.38 \\
\hline 3.18 & 0.24 & 0.15 & 0.14 & 0.13 & 0.16 & 97.50 \\
\hline 2.75 & 0.18 & 0.09 & 0.04 & 0.07 & 0.15 & 98.08 \\
\hline 2.71 & 0.18 & 0.15 & 0.03 & 0.08 & 0.17 & 98.16 \\
\hline 2.87 & 0.19 & 0.12 & 0.02 & 0.10 & 0.20 & 98.24 \\
\hline 2.84 & 0.19 & 0.09 & 0.00 & 0.10 & 0.18 & 98.15 \\
\hline 2.86 & 0.20 & 0.09 & 0.13 & 0.08 & 0.21 & 98.10 \\
\hline 2.76 & 0.18 & 0.09 & 0.00 & 0.08 & 0.16 & 97.55 \\
\hline 2.88 & 0.17 & 0.10 & 0.00 & 0.09 & 0.15 & 97.98 \\
\hline 2.85 & 0.18 & 0.08 & 0.06 & 0.09 & 0.15 & 98.14 \\
\hline 2.90 & 0.18 & 0.11 & 0.00 & 0.08 & 0.22 & 98.27 \\
\hline 2.79 & 0.18 & 0.11 & 0.02 & 0.09 & 0.17 & 97.52 \\
\hline 2.85 & 0.19 & 0.12 & 0.00 & 0.08 & 0.17 & 98.00 \\
\hline 2.78 & 0.18 & 0.11 & 0.00 & 0.09 & 0.19 & 97.21 \\
\hline 2.81 & 0.19 & 0.11 & 0.02 & 0.07 & 0.18 & 97.82 \\
\hline 2.67 & 0.18 & 0.10 & 0.09 & 0.07 & 0.20 & 97.23 \\
\hline 2.76 & 0.18 & 0.07 & 0.00 & 0.09 & 0.18 & 97.26 \\
\hline 2.84 & 0.19 & 0.08 & 0.00 & 0.07 & 0.19 & 98.38 \\
\hline 2.77 & 0.19 & 0.13 & 0.04 & 0.09 & 0.19 & 97.92 \\
\hline 2.91 & 0.19 & 0.15 & 0.00 & 0.07 & 0.17 & 98.50 \\
\hline 2.79 & 0.18 & 0.10 & 0.00 & 0.09 & 0.19 & 98.23 \\
\hline 2.82 & 0.18 & 0.12 & 0.00 & 0.09 & 0.18 & 97.49 \\
\hline 2.86 & 0.18 & 0.06 & 0.06 & 0.09 & 0.22 & 98.26 \\
\hline 2.80 & 0.21 & 0.12 & 0.00 & 0.08 & 0.18 & 97.83 \\
\hline 2.74 & 0.19 & 0.10 & 0.00 & 0.09 & 0.20 & 97.96 \\
\hline 2.85 & 0.18 & 0.14 & 0.00 & 0.08 & 0.20 & 98.42 \\
\hline 2.81 & 0.20 & 0.08 & 0.01 & 0.09 & 0.18 & 97.32 \\
\hline 2.85 & 0.19 & 0.08 & 0.00 & 0.08 & 0.20 & 97.72 \\
\hline 2.80 & 0.19 & 0.08 & 0.01 & 0.09 & 0.16 & 97.85 \\
\hline 2.86 & 0.19 & 0.09 & 0.00 & 0.07 & 0.17 & 97.71 \\
\hline 2.70 & 0.19 & 0.09 & 0.00 & 0.08 & 0.19 & 98.08 \\
\hline 2.80 & 0.18 & 0.10 & 0.17 & 0.09 & 0.19 & 97.87 \\
\hline 3.18 & 0.28 & 0.11 & 0.00 & 0.15 & 0.11 & 96.49 \\
\hline
\end{tabular}




\begin{tabular}{|c|c|c|c|c|c|c|}
\hline 3.22 & 0.25 & 0.09 & 0.00 & 0.16 & 0.15 & 96.90 \\
\hline 2.76 & 0.29 & 0.06 & 0.00 & 0.17 & 0.13 & 95.76 \\
\hline 3.25 & 0.26 & 0.11 & 0.00 & 0.17 & 0.18 & 96.63 \\
\hline 3.25 & 0.27 & 0.11 & 0.04 & 0.17 & 0.15 & 97.05 \\
\hline 2.56 & 0.24 & 0.16 & 0.00 & 0.15 & 0.37 & 94.18 \\
\hline 3.09 & 0.26 & 0.14 & 0.00 & 0.15 & 0.16 & 96.75 \\
\hline 3.10 & 0.26 & 0.15 & 0.00 & 0.17 & 0.16 & 97.15 \\
\hline 3.15 & 0.28 & 0.11 & 0.01 & 0.15 & 0.16 & 96.85 \\
\hline 3.16 & 0.24 & 0.15 & 0.00 & 0.15 & 0.15 & 96.89 \\
\hline 3.12 & 0.26 & 0.09 & 0.00 & 0.17 & 0.13 & 97.00 \\
\hline 3.24 & 0.26 & 0.14 & 0.00 & 0.16 & 0.13 & 96.98 \\
\hline 3.23 & 0.25 & 0.14 & 0.00 & 0.16 & 0.15 & 96.48 \\
\hline 3.16 & 0.25 & 0.16 & 0.08 & 0.14 & 0.22 & 97.31 \\
\hline 3.03 & 0.25 & 0.14 & 0.05 & 0.12 & 0.17 & 97.45 \\
\hline 3.15 & 0.27 & 0.17 & 0.00 & 0.12 & 0.20 & 97.72 \\
\hline 3.17 & 0.27 & 0.11 & 0.00 & 0.13 & 0.19 & 97.44 \\
\hline 3.17 & 0.26 & 0.14 & 0.00 & 0.14 & 0.19 & 97.41 \\
\hline 3.16 & 0.26 & 0.11 & 0.00 & 0.13 & 0.24 & 97.58 \\
\hline 2.84 & 0.39 & 0.14 & 0.00 & 0.15 & 0.21 & 95.53 \\
\hline 3.06 & 0.27 & 0.09 & 0.00 & 0.13 & 0.19 & 97.40 \\
\hline 3.26 & 0.26 & 0.13 & 0.00 & 0.14 & 0.19 & 97.70 \\
\hline 3.18 & 0.26 & 0.15 & 0.00 & 0.13 & 0.20 & 97.55 \\
\hline 3.03 & 0.23 & 0.12 & 0.00 & 0.12 & 0.19 & 97.53 \\
\hline 3.22 & 0.24 & 0.15 & 0.07 & 0.11 & 0.21 & 97.19 \\
\hline 3.04 & 0.26 & 0.10 & 0.00 & 0.11 & 0.19 & 97.05 \\
\hline 3.19 & 0.25 & 0.15 & 0.00 & 0.12 & 0.19 & 97.59 \\
\hline 3.07 & 0.26 & 0.13 & 0.10 & 0.13 & 0.22 & 97.33 \\
\hline 3.19 & 0.25 & 0.11 & 0.00 & 0.12 & 0.20 & 97.49 \\
\hline 3.16 & 0.26 & 0.13 & 0.02 & 0.13 & 0.18 & 97.59 \\
\hline 3.30 & 0.26 & 0.13 & 0.02 & 0.12 & 0.17 & 97.44 \\
\hline 3.07 & 0.26 & 0.10 & 0.07 & 0.13 & 0.17 & 97.56 \\
\hline 3.13 & 0.25 & 0.08 & 0.00 & 0.15 & 0.16 & 96.59 \\
\hline 3.05 & 0.24 & 0.10 & 0.00 & 0.13 & 0.21 & 96.79 \\
\hline 3.04 & 0.26 & 0.14 & 0.00 & 0.14 & 0.19 & 97.18 \\
\hline 2.91 & 0.24 & 0.14 & 0.00 & 0.14 & 0.18 & 96.45 \\
\hline 3.20 & 0.25 & 0.13 & 0.00 & 0.14 & 0.22 & 97.33 \\
\hline 3.19 & 0.26 & 0.15 & 0.00 & 0.12 & 0.17 & 97.67 \\
\hline 2.95 & 0.25 & 0.14 & 0.00 & 0.14 & 0.20 & 97.39 \\
\hline 3.14 & 0.25 & 0.14 & 0.10 & 0.14 & 0.20 & 96.82 \\
\hline 3.02 & 0.25 & 0.11 & 0.00 & 0.12 & 0.17 & 96.85 \\
\hline 3.17 & 0.24 & 0.13 & 0.02 & 0.11 & 0.19 & 97.82 \\
\hline 3.27 & 0.25 & & 0.00 & 0.14 & 0.18 & 96.93 \\
\hline 3.21 & 0.26 & & 0.01 & 0.16 & 0.17 & 97.22 \\
\hline 3.28 & 0.24 & & 0.00 & 0.16 & 0.16 & 97.51 \\
\hline 3.28 & 0.25 & & 0.17 & 0.13 & 0.21 & 97.36 \\
\hline 3.31 & 0.25 & & 0.00 & 0.12 & 0.21 & 97.32 \\
\hline 3.32 & 0.24 & & 0.00 & 0.13 & 0.16 & 97.37 \\
\hline
\end{tabular}




\begin{tabular}{|c|c|c|c|c|c|}
\hline 3.31 & 0.26 & 0.00 & 0.14 & 0.20 & 96.48 \\
\hline 3.33 & 0.24 & 0.00 & 0.14 & 0.19 & 97.17 \\
\hline 3.09 & 0.26 & 0.00 & 0.14 & 0.19 & 97.11 \\
\hline 3.30 & 0.23 & 0.00 & 0.14 & 0.16 & 97.03 \\
\hline 3.38 & 0.25 & 0.14 & 0.22 & 0.18 & 96.47 \\
\hline 3.24 & 0.28 & 0.00 & 0.23 & 0.15 & 96.60 \\
\hline 3.49 & 0.27 & 0.07 & 0.19 & 0.18 & 96.75 \\
\hline 3.37 & 0.27 & 0.00 & 0.20 & 0.17 & 97.03 \\
\hline 2.99 & 0.25 & 0.00 & 0.19 & 0.18 & 96.51 \\
\hline 3.35 & 0.27 & 0.00 & 0.18 & 0.19 & 96.98 \\
\hline 3.28 & 0.25 & 0.00 & 0.18 & 0.16 & 96.79 \\
\hline 3.25 & 0.25 & 0.00 & 0.16 & 0.18 & 96.27 \\
\hline 3.23 & 0.25 & 0.00 & 0.16 & 0.18 & 96.58 \\
\hline 3.29 & 0.26 & 0.02 & 0.19 & 0.17 & 96.89 \\
\hline 2.91 & 0.18 & 0.10 & 0.10 & 0.21 & 97.99 \\
\hline 2.87 & 0.17 & 0.03 & 0.08 & 0.21 & 97.49 \\
\hline 2.76 & 0.19 & 0.14 & 0.07 & 0.18 & 97.96 \\
\hline 2.64 & 0.18 & 0.00 & 0.09 & 0.19 & 97.49 \\
\hline 2.74 & 0.17 & 0.12 & 0.08 & 0.19 & 97.53 \\
\hline 2.82 & 0.19 & 0.00 & 0.08 & 0.17 & 97.60 \\
\hline 2.92 & 0.17 & 0.06 & 0.08 & 0.22 & 97.93 \\
\hline 2.64 & 0.18 & 0.00 & 0.09 & 0.17 & 97.82 \\
\hline 2.94 & 0.18 & 0.00 & 0.09 & 0.19 & 98.15 \\
\hline 3.32 & 0.25 & 0.07 & 0.16 & 0.16 & 97.16 \\
\hline 3.34 & 0.27 & 0.00 & 0.18 & 0.15 & 96.98 \\
\hline 3.26 & 0.25 & 0.03 & 0.17 & 0.14 & 96.82 \\
\hline 3.26 & 0.26 & 0.05 & 0.16 & 0.16 & 97.11 \\
\hline 3.35 & 0.25 & 0.00 & 0.17 & 0.15 & 96.92 \\
\hline 3.26 & 0.25 & 0.06 & 0.15 & 0.16 & 96.55 \\
\hline 3.31 & 0.27 & 0.13 & 0.16 & 0.16 & 96.00 \\
\hline 3.19 & 0.24 & 0.00 & 0.17 & 0.17 & 95.94 \\
\hline 3.17 & 0.26 & 0.00 & 0.18 & 0.17 & 96.81 \\
\hline 3.39 & 0.26 & 0.00 & 0.16 & 0.20 & 97.04 \\
\hline 3.30 & 0.25 & 0.01 & 0.13 & 0.24 & 97.91 \\
\hline 3.32 & 0.26 & 0.00 & 0.12 & 0.21 & 97.04 \\
\hline 3.14 & 0.26 & 0.00 & 0.15 & 0.20 & 97.63 \\
\hline 3.33 & 0.25 & 0.00 & 0.15 & 0.22 & 97.40 \\
\hline 3.20 & 0.26 & 0.09 & 0.14 & 0.21 & 97.17 \\
\hline 3.32 & 0.24 & 0.00 & 0.13 & 0.19 & 96.91 \\
\hline 3.29 & 0.27 & 0.00 & 0.14 & 0.18 & 97.25 \\
\hline 3.19 & 0.25 & 0.00 & 0.13 & 0.22 & 97.05 \\
\hline 3.32 & 0.25 & 0.00 & 0.13 & 0.19 & 97.02 \\
\hline 2.97 & 0.24 & 0.05 & 0.12 & 0.15 & 98.14 \\
\hline
\end{tabular}




\begin{tabular}{|c|c|c|c|c|c|c|c|c|c|}
\hline $4 / 23 / 2014$ & $\mathrm{SiO} 2$ & TiO2 & Al2O3 & $\mathrm{Cr} 2 \mathrm{O} 3$ & $\mathrm{FeO}$ & $\mathrm{MnO}$ & $\mathrm{MgO}$ & $\mathrm{CaO}$ & $\mathrm{Na} 2 \mathrm{O}$ \\
\hline R15-11 & 52.11 & 1.13 & 16.48 & 0.00 & 8.80 & 0.17 & 5.40 & 9.78 & 3.05 \\
\hline R15-11 & 52.02 & 1.13 & 16.41 & 0.00 & 8.85 & 0.18 & 5.40 & 10.02 & 3.18 \\
\hline R15-11 & 51.88 & 1.11 & 16.51 & 0.00 & 8.88 & 0.17 & 5.28 & 10.18 & 3.07 \\
\hline R15-11 & 52.04 & 1.13 & 16.39 & 0.00 & 8.72 & 0.15 & 5.38 & 10.07 & 3.18 \\
\hline R15-11 & 52.10 & 1.13 & 16.44 & 0.01 & 8.89 & 0.15 & 5.32 & 9.91 & 3.20 \\
\hline R15-11 & 52.11 & 1.16 & 16.55 & 0.00 & 8.97 & 0.17 & 5.34 & 9.83 & 3.02 \\
\hline R15-11 & 52.04 & 1.12 & 16.29 & 0.00 & 8.79 & 0.17 & 5.48 & 9.80 & 3.16 \\
\hline R15-11 & 51.96 & 1.15 & 16.50 & 0.00 & 8.67 & 0.19 & 5.45 & 10.00 & 3.16 \\
\hline R15-11 & 51.71 & 1.14 & 16.45 & 0.00 & 8.87 & 0.16 & 5.48 & 10.13 & 3.14 \\
\hline R15-11 & 52.05 & 1.15 & 16.43 & 0.00 & 8.89 & 0.15 & 5.44 & 10.11 & 3.15 \\
\hline R15-11 & 51.92 & 1.15 & 16.16 & 0.00 & 8.81 & 0.18 & 5.36 & 10.16 & 3.23 \\
\hline R15-11 & 51.99 & 1.17 & 16.34 & 0.02 & 8.88 & 0.16 & 5.41 & 10.07 & 3.13 \\
\hline R15-11 & 51.99 & 1.15 & 16.45 & 0.00 & 8.79 & 0.19 & 5.36 & 10.09 & 3.26 \\
\hline R15-11 & 52.00 & 1.14 & 16.39 & 0.00 & 8.86 & 0.15 & 5.42 & 10.13 & 3.20 \\
\hline R15-11 & 52.09 & 1.18 & 16.48 & 0.00 & 8.99 & 0.16 & 5.35 & 10.03 & 3.15 \\
\hline R15-12r & 52.02 & 1.19 & 16.42 & 0.00 & 8.97 & 0.15 & 5.49 & 10.10 & 3.22 \\
\hline R15-12r & 52.09 & 1.07 & 16.12 & 0.00 & 8.98 & 0.15 & 5.43 & 10.00 & 3.29 \\
\hline R15-12r & 52.24 & 1.11 & 16.44 & 0.00 & 9.00 & 0.17 & 5.33 & 10.20 & 3.20 \\
\hline R15-12r & 52.02 & 1.14 & 16.38 & 0.00 & 8.72 & 0.18 & 5.51 & 10.00 & 3.23 \\
\hline R15-12r & 51.59 & 1.19 & 16.29 & 0.01 & 8.87 & 0.16 & 5.60 & 10.32 & 2.89 \\
\hline R15-12r & 51.94 & 1.14 & 16.60 & 0.03 & 8.78 & 0.19 & 5.39 & 10.04 & 3.26 \\
\hline R15-12r & 51.01 & 1.14 & 16.33 & 0.01 & 9.00 & 0.16 & 5.43 & 10.08 & 3.19 \\
\hline R15-12r & 51.97 & 1.14 & 16.49 & 0.01 & 8.89 & 0.15 & 5.40 & 10.10 & 3.16 \\
\hline R15-12r & 52.12 & 1.12 & 16.48 & 0.03 & 9.00 & 0.14 & 5.46 & 9.87 & 2.96 \\
\hline R15-12r & 52.21 & 1.13 & 16.38 & 0.00 & 9.03 & 0.19 & 5.40 & 9.94 & 3.24 \\
\hline R15-12r & 52.21 & 1.12 & 16.50 & 0.00 & 8.96 & 0.16 & 5.42 & 10.13 & 3.16 \\
\hline R15-12r & 51.39 & 1.14 & 16.42 & 0.01 & 9.01 & 0.17 & 5.44 & 9.97 & 3.17 \\
\hline$R 15-12 r$ & 51.87 & 1.15 & 16.50 & 0.00 & 8.91 & 0.14 & 5.32 & 10.07 & 3.21 \\
\hline R15-13r & 52.08 & 1.16 & 16.61 & 0.01 & 8.90 & 0.15 & 5.47 & 9.49 & 3.20 \\
\hline R15-13r & 51.91 & 1.15 & 16.45 & 0.00 & 9.05 & 0.17 & 5.52 & 10.05 & 3.24 \\
\hline R15-13r & 51.90 & 1.14 & 16.50 & 0.00 & 8.75 & 0.17 & 5.09 & 9.96 & 3.24 \\
\hline R15-13r & 52.18 & 1.17 & 16.20 & 0.00 & 8.92 & 0.16 & 5.35 & 10.01 & 3.15 \\
\hline R15-13r & 52.20 & 1.15 & 16.55 & 0.01 & 8.99 & 0.18 & 5.43 & 10.16 & 3.15 \\
\hline R15-13r & 51.92 & 1.14 & 16.50 & 0.00 & 8.91 & 0.18 & 5.34 & 10.19 & 3.10 \\
\hline R15-13r & 51.90 & 1.15 & 16.50 & 0.00 & 9.00 & 0.19 & 5.29 & 9.99 & 3.11 \\
\hline R15-13r & 51.96 & 1.15 & 16.42 & 0.02 & 9.05 & 0.15 & 5.50 & 9.84 & 3.15 \\
\hline R15-13r & 51.99 & 1.10 & 16.41 & 0.01 & 9.03 & 0.17 & 5.48 & 10.18 & 3.21 \\
\hline R15-13r & 52.12 & 1.15 & 16.49 & 0.00 & 9.03 & 0.16 & 5.44 & 9.96 & 3.08 \\
\hline R15-13r & 52.06 & 1.21 & 16.52 & 0.00 & 8.89 & 0.16 & 5.31 & 10.16 & 3.22 \\
\hline R15-13r & 52.09 & 1.17 & 16.50 & 0.01 & 8.85 & 0.18 & 5.44 & 10.06 & 3.25 \\
\hline R15-13r & 52.13 & 1.14 & 16.49 & 0.01 & 8.95 & 0.19 & 5.43 & 10.15 & 3.13 \\
\hline R15-13r & 52.19 & 1.15 & 16.53 & 0.00 & 9.01 & 0.15 & 5.42 & 9.96 & 3.17 \\
\hline R15-13r & 51.57 & 1.16 & 16.47 & 0.00 & 8.77 & 0.18 & 5.40 & 10.03 & 3.24 \\
\hline R15-14r & 51.43 & 1.19 & 15.92 & 0.01 & 8.80 & 0.17 & 5.28 & 9.65 & 3.31 \\
\hline R15-14r & 52.13 & 1.23 & 16.38 & 0.00 & 8.95 & 0.15 & 5.11 & 10.01 & 3.16 \\
\hline R15-14r & 52.04 & 1.13 & 16.45 & 0.02 & 8.85 & 0.17 & 5.17 & 10.06 & 3.22 \\
\hline
\end{tabular}




\begin{tabular}{|c|c|c|c|c|c|c|c|c|c|}
\hline R15-14r & 51.51 & 0.87 & 20.90 & 0.00 & 6.80 & 0.13 & 3.78 & 10.66 & 3.26 \\
\hline R15-14r & 52.03 & 1.14 & 16.42 & 0.00 & 8.81 & 0.15 & 5.23 & 9.96 & 3.22 \\
\hline R15-14r & 52.10 & 1.17 & 16.36 & 0.02 & 8.77 & 0.19 & 5.16 & 10.14 & 3.00 \\
\hline R15-14r & 52.15 & 1.15 & 16.52 & 0.00 & 8.91 & 0.14 & 5.17 & 10.05 & 3.16 \\
\hline R15-14r & 51.97 & 1.14 & 16.27 & 0.02 & 8.87 & 0.19 & 5.28 & 9.99 & 3.15 \\
\hline$R 15-14 r$ & 51.84 & 1.17 & 16.14 & 0.01 & 8.91 & 0.15 & 5.22 & 10.11 & 3.14 \\
\hline R15-14r & 51.99 & 1.10 & 16.48 & 0.00 & 8.49 & 0.16 & 5.21 & 10.15 & 3.16 \\
\hline R15-14r & 51.88 & 1.14 & 16.13 & 0.01 & 8.78 & 0.17 & 5.53 & 10.38 & 3.14 \\
\hline R15-14r & 51.84 & 1.15 & 16.09 & 0.01 & 8.83 & 0.14 & 5.26 & 9.87 & 3.17 \\
\hline R15-14r & 51.83 & 1.18 & 16.27 & 0.00 & 8.91 & 0.15 & 5.23 & 10.01 & 3.05 \\
\hline R15-15r & 52.14 & 1.12 & 16.28 & 0.00 & 8.83 & 0.17 & 5.32 & 9.96 & 3.23 \\
\hline R15-15r & 52.01 & 1.17 & 16.27 & 0.01 & 8.80 & 0.18 & 5.40 & 10.10 & 3.15 \\
\hline R15-15r & 51.94 & 1.20 & 16.47 & 0.00 & 8.92 & 0.16 & 5.45 & 10.04 & 3.16 \\
\hline R15-15r & 52.04 & 1.17 & 16.39 & 0.01 & 8.94 & 0.16 & 5.24 & 9.98 & 3.21 \\
\hline R15-15r & 50.85 & 1.18 & 16.53 & 0.00 & 8.84 & 0.16 & 5.34 & 10.06 & 3.21 \\
\hline$R 15-15 r$ & 52.08 & 1.15 & 16.47 & 0.01 & 8.84 & 0.16 & 5.38 & 9.91 & 3.27 \\
\hline R15-15r & 51.73 & 1.14 & 15.99 & 0.00 & 8.96 & 0.17 & 5.32 & 10.02 & 3.19 \\
\hline R15-15r & 52.21 & 1.10 & 16.53 & 0.02 & 8.97 & 0.17 & 5.43 & 10.06 & 3.16 \\
\hline R15-15r & 51.87 & 1.17 & 16.39 & 0.01 & 8.90 & 0.16 & 5.38 & 9.90 & 3.28 \\
\hline R15-15r & 51.94 & 1.23 & 16.26 & 0.02 & 8.74 & 0.16 & 5.40 & 10.09 & 3.23 \\
\hline R15-15r & 51.90 & 1.14 & 16.46 & 0.00 & 9.07 & 0.16 & 5.36 & 10.01 & 3.18 \\
\hline R15-15r & 51.90 & 1.12 & 16.42 & 0.00 & 8.98 & 0.14 & 5.12 & 10.00 & 3.19 \\
\hline$R 15-15 r$ & 52.01 & 1.16 & 16.52 & 0.01 & 8.92 & 0.15 & 5.37 & 9.71 & 3.25 \\
\hline R15-15r & 52.03 & 1.12 & 16.52 & 0.02 & 9.03 & 0.17 & 5.36 & 10.00 & 3.14 \\
\hline R15-15r & 51.97 & 1.17 & 16.55 & 0.00 & 8.88 & 0.16 & 5.39 & 10.08 & 3.19 \\
\hline R15-16r & 52.17 & 1.13 & 16.53 & 0.00 & 9.08 & 0.17 & 5.32 & 10.12 & 3.08 \\
\hline R15-16r & 52.03 & 1.20 & 16.64 & 0.00 & 8.77 & 0.15 & 5.22 & 9.93 & 3.24 \\
\hline R15-16r & 51.98 & 1.11 & 15.90 & 0.02 & 8.86 & 0.17 & 5.86 & 10.58 & 2.88 \\
\hline R15-16r & 52.20 & 1.09 & 16.41 & 0.00 & 9.00 & 0.15 & 5.25 & 9.96 & 3.12 \\
\hline R15-16r & 52.03 & 1.14 & 16.32 & 0.00 & 8.95 & 0.18 & 5.35 & 9.98 & 3.15 \\
\hline$R 15-16 r$ & 52.19 & 1.13 & 16.33 & 0.01 & 8.71 & 0.17 & 5.33 & 9.98 & 3.29 \\
\hline R15-16r & 52.11 & 1.13 & 16.52 & 0.01 & 8.95 & 0.15 & 5.25 & 9.87 & 3.25 \\
\hline R15-16r & 52.24 & 1.15 & 16.43 & 0.01 & 8.64 & 0.18 & 5.37 & 10.04 & 3.28 \\
\hline R15-16r & 51.89 & 1.14 & 16.33 & 0.00 & 8.94 & 0.19 & 5.02 & 9.77 & 3.21 \\
\hline R15-16r & 51.83 & 1.10 & 16.45 & 0.00 & 8.98 & 0.20 & 5.35 & 9.85 & 3.25 \\
\hline R15-16r & 51.92 & 1.25 & 16.44 & 0.00 & 8.90 & 0.19 & 5.23 & 10.02 & 3.12 \\
\hline R15-16r & 52.16 & 1.21 & 16.44 & 0.02 & 9.03 & 0.19 & 5.42 & 10.04 & 3.20 \\
\hline R15-16r & 51.90 & 1.15 & 16.46 & 0.00 & 8.59 & 0.16 & 5.13 & 9.96 & 3.13 \\
\hline R15-16r & 51.26 & 1.16 & 15.47 & 0.01 & 9.00 & 0.16 & 6.16 & 11.47 & 2.87 \\
\hline R15-16r & 51.94 & 1.14 & 16.42 & 0.00 & 8.85 & 0.19 & 5.15 & 10.14 & 3.21 \\
\hline R15-12 & 52.20 & 1.16 & 15.95 & 0.00 & 9.01 & 0.16 & 5.46 & 10.04 & 3.29 \\
\hline R15-12 & 51.98 & 1.13 & 16.23 & 0.01 & 8.92 & 0.16 & 5.45 & 9.95 & 3.15 \\
\hline R15-12 & 51.57 & 1.16 & 16.20 & 0.00 & 9.00 & 0.16 & 6.04 & 9.62 & 3.21 \\
\hline R15-12 & 52.01 & 1.11 & 16.41 & 0.02 & 8.72 & 0.18 & 5.27 & 10.01 & 3.36 \\
\hline R15-12 & 51.50 & 0.90 & 20.40 & 0.02 & 6.58 & 0.14 & 4.16 & 11.01 & 3.30 \\
\hline R15-12 & 52.16 & 1.16 & 16.32 & 0.00 & 8.94 & 0.15 & 5.36 & 9.94 & 3.33 \\
\hline R15-12 & 52.06 & 1.17 & 16.34 & 0.00 & 8.84 & 0.19 & 5.39 & 9.97 & 3.34 \\
\hline
\end{tabular}




\begin{tabular}{|c|c|c|c|c|c|c|c|c|c|}
\hline R15-12 & 51.92 & 1.15 & 16.40 & 0.01 & 8.86 & 0.17 & 5.45 & 10.03 & 3.22 \\
\hline R15-12 & 52.31 & 1.15 & 16.35 & 0.00 & 8.99 & 0.16 & 5.42 & 9.99 & 3.30 \\
\hline R15-12 & 52.33 & 1.12 & 16.42 & 0.00 & 9.09 & 0.16 & 5.51 & 9.96 & 3.15 \\
\hline R15-14 & 51.91 & 1.10 & 17.53 & 0.00 & 8.29 & 0.16 & 4.87 & 10.44 & 3.35 \\
\hline R15-14 & 51.98 & 0.99 & 18.06 & 0.02 & 7.77 & 0.16 & 5.01 & 10.75 & 3.32 \\
\hline R15-14 & 51.67 & 1.10 & 16.14 & 0.01 & 8.96 & 0.16 & 5.31 & 10.05 & 3.26 \\
\hline R15-14 & 51.86 & 1.14 & 16.34 & 0.02 & 8.51 & 0.16 & 5.20 & 9.93 & 3.21 \\
\hline R15-14 & 52.03 & 1.15 & 16.22 & 0.00 & 8.88 & 0.17 & 5.45 & 9.89 & 3.45 \\
\hline R15-14 & 51.89 & 1.16 & 15.79 & 0.00 & 8.98 & 0.15 & 5.33 & 10.01 & 3.22 \\
\hline R15-14 & 51.90 & 1.22 & 16.46 & 0.01 & 8.88 & 0.15 & 5.09 & 10.01 & 3.35 \\
\hline R15-14 & 51.75 & 1.21 & 16.41 & 0.01 & 8.83 & 0.16 & 5.26 & 10.05 & 3.31 \\
\hline R15-14 & 51.60 & 1.17 & 15.50 & 0.00 & 8.90 & 0.17 & 5.37 & 9.36 & 3.29 \\
\hline R15-14 & 51.87 & 1.14 & 16.26 & 0.01 & 8.98 & 0.17 & 5.43 & 9.95 & 3.25 \\
\hline R15-15 & 52.04 & 1.14 & 16.52 & 0.01 & 9.02 & 0.16 & 5.46 & 9.82 & 3.27 \\
\hline R15-15 & 51.81 & 1.21 & 16.36 & 0.01 & 9.04 & 0.17 & 5.43 & 9.96 & 3.23 \\
\hline R15-15 & 52.11 & 1.21 & 16.31 & 0.00 & 8.96 & 0.11 & 5.52 & 10.00 & 3.21 \\
\hline R15-15 & 52.06 & 1.14 & 15.92 & 0.00 & 8.98 & 0.14 & 5.43 & 9.99 & 3.16 \\
\hline R15-15 & 51.62 & 1.11 & 16.26 & 0.00 & 8.92 & 0.19 & 5.34 & 9.80 & 3.26 \\
\hline R15-15 & 52.13 & 1.21 & 16.38 & 0.00 & 9.00 & 0.16 & 5.29 & 9.93 & 3.26 \\
\hline R15-15 & 52.01 & 1.13 & 16.30 & 0.00 & 8.96 & 0.18 & 5.26 & 9.91 & 3.02 \\
\hline R15-15 & 52.08 & 1.15 & 16.33 & 0.02 & 8.95 & 0.15 & 5.27 & 9.94 & 3.31 \\
\hline R15-15 & 51.54 & 1.14 & 15.67 & 0.00 & 9.59 & 0.16 & 7.43 & 9.25 & 3.19 \\
\hline R15-15 & 52.02 & 1.14 & 16.49 & 0.00 & 8.88 & 0.18 & 5.36 & 9.96 & 3.33 \\
\hline R15-16 & 52.03 & 1.18 & 16.70 & 0.00 & 8.93 & 0.16 & 4.92 & 9.74 & 3.37 \\
\hline R15-16 & 51.46 & 1.10 & 15.67 & 0.00 & 9.26 & 0.15 & 6.68 & 9.42 & 3.13 \\
\hline R15-16 & 51.92 & 1.13 & 17.18 & 0.00 & 8.67 & 0.14 & 4.96 & 9.89 & 3.20 \\
\hline R15-16 & 51.23 & 1.11 & 15.80 & 0.00 & 9.12 & 0.14 & 7.08 & 9.30 & 3.11 \\
\hline R15-16 & 52.07 & 1.17 & 16.52 & 0.00 & 8.99 & 0.20 & 5.14 & 9.72 & 3.29 \\
\hline R15-16 & 52.17 & 1.01 & 17.92 & 0.01 & 8.09 & 0.17 & 4.71 & 10.20 & 3.33 \\
\hline R15-16 & 52.21 & 1.18 & 16.55 & 0.01 & 8.98 & 0.16 & 4.87 & 9.94 & 3.40 \\
\hline R15-16 & 51.91 & 1.19 & 16.51 & 0.01 & 8.89 & 0.20 & 5.04 & 9.86 & 3.27 \\
\hline
\end{tabular}




\begin{tabular}{|c|c|c|c|c|c|}
\hline $\mathrm{K} 2 \mathrm{O}$ & P2O5 & $\mathrm{F}$ & $\mathrm{Cl}$ & $\mathrm{SO} 3$ & Total \\
\hline 0.27 & 0.11 & 0.08 & 0.13 & 0.22 & 97.68 \\
\hline 0.25 & 0.08 & 0.06 & 0.11 & 0.20 & 97.84 \\
\hline 0.26 & 0.09 & 0.00 & 0.12 & 0.17 & 97.68 \\
\hline 0.25 & 0.12 & 0.00 & 0.12 & 0.17 & 97.68 \\
\hline 0.26 & 0.15 & 0.00 & 0.12 & 0.19 & 97.83 \\
\hline 0.24 & 0.12 & 0.00 & 0.14 & 0.21 & 97.84 \\
\hline 0.26 & 0.08 & 0.00 & 0.11 & 0.17 & 97.46 \\
\hline 0.26 & 0.18 & 0.03 & 0.12 & 0.19 & 97.81 \\
\hline 0.26 & 0.12 & 0.00 & 0.14 & 0.18 & 97.75 \\
\hline 0.24 & 0.08 & 0.00 & 0.13 & 0.19 & 97.98 \\
\hline 0.24 & 0.15 & 0.03 & 0.12 & 0.18 & 97.64 \\
\hline 0.27 & 0.12 & 0.14 & 0.12 & 0.19 & 97.93 \\
\hline 0.26 & 0.09 & 0.04 & 0.12 & 0.22 & 97.95 \\
\hline 0.24 & 0.14 & 0.06 & 0.12 & 0.20 & 98.00 \\
\hline 0.27 & 0.17 & 0.00 & 0.12 & 0.18 & 98.14 \\
\hline 0.25 & 0.16 & 0.00 & 0.13 & 0.17 & 98.23 \\
\hline 0.26 & 0.12 & 0.00 & 0.15 & 0.18 & 97.79 \\
\hline 0.25 & 0.09 & 0.18 & 0.12 & 0.23 & 98.47 \\
\hline 0.26 & 0.09 & 0.15 & 0.13 & 0.21 & 97.93 \\
\hline 0.27 & 0.12 & 0.00 & 0.14 & 0.21 & 97.61 \\
\hline 0.26 & 0.11 & 0.03 & 0.14 & 0.20 & 98.06 \\
\hline 0.27 & 0.15 & 0.00 & 0.14 & 0.20 & 97.07 \\
\hline 0.27 & 0.13 & 0.00 & 0.13 & 0.22 & 98.04 \\
\hline 0.27 & 0.13 & 0.11 & 0.13 & 0.19 & 97.92 \\
\hline 0.27 & 0.12 & 0.00 & 0.14 & 0.20 & 98.22 \\
\hline 0.26 & 0.10 & 0.10 & 0.13 & 0.18 & 98.37 \\
\hline 0.28 & 0.16 & 0.04 & 0.13 & 0.20 & 97.49 \\
\hline 0.27 & 0.13 & 0.00 & 0.14 & 0.17 & 97.84 \\
\hline 0.27 & 0.11 & 0.05 & 0.13 & 0.19 & 97.77 \\
\hline 0.27 & 0.14 & 0.00 & 0.14 & 0.23 & 98.27 \\
\hline 0.26 & 0.18 & 0.00 & 0.11 & 0.20 & 97.48 \\
\hline 0.26 & 0.11 & 0.00 & 0.11 & 0.17 & 97.78 \\
\hline 0.25 & 0.16 & 0.04 & 0.13 & 0.20 & 98.55 \\
\hline 0.26 & 0.15 & 0.14 & 0.13 & 0.20 & 98.07 \\
\hline 0.27 & 0.13 & 0.00 & 0.13 & 0.20 & 97.83 \\
\hline 0.28 & 0.13 & 0.08 & 0.13 & 0.20 & 98.02 \\
\hline 0.24 & 0.14 & 0.00 & 0.12 & 0.20 & 98.25 \\
\hline 0.26 & 0.13 & 0.00 & 0.12 & 0.20 & 98.09 \\
\hline 0.27 & 0.10 & 0.01 & 0.10 & 0.21 & 98.19 \\
\hline 0.26 & 0.17 & 0.00 & 0.13 & 0.21 & 98.29 \\
\hline 0.26 & 0.13 & 0.00 & 0.12 & 0.21 & 98.30 \\
\hline 0.25 & 0.14 & 0.03 & 0.12 & 0.19 & 98.27 \\
\hline 0.25 & 0.14 & 0.01 & 0.11 & 0.20 & 97.52 \\
\hline 0.28 & 0.07 & 0.00 & 0.13 & 0.21 & 96.42 \\
\hline 0.27 & 0.09 & 0.00 & 0.13 & 0.19 & 97.78 \\
\hline 0.27 & 0.15 & 0.00 & 0.13 & 0.18 & 97.79 \\
\hline
\end{tabular}




$\begin{array}{llllll}0.20 & 0.10 & 0.04 & 0.10 & 0.14 & 98.44 \\ 0.26 & 0.09 & 0.07 & 0.12 & 0.20 & 97.64 \\ 0.27 & 0.13 & 0.00 & 0.13 & 0.18 & 97.60 \\ 0.25 & 0.13 & 0.01 & 0.13 & 0.19 & 97.92 \\ 0.26 & 0.12 & 0.00 & 0.14 & 0.21 & 97.57 \\ 0.27 & 0.13 & 0.00 & 0.12 & 0.16 & 97.36 \\ 0.26 & 0.12 & 0.09 & 0.12 & 0.22 & 97.48 \\ 0.26 & 0.14 & 0.09 & 0.13 & 0.20 & 97.90 \\ 0.25 & 0.09 & 0.00 & 0.13 & 0.19 & 96.97 \\ 0.27 & 0.10 & 0.00 & 0.13 & 0.20 & 97.30 \\ 0.24 & 0.15 & 0.18 & 0.13 & 0.19 & 97.82 \\ 0.27 & 0.07 & 0.00 & 0.13 & 0.19 & 97.73 \\ 0.26 & 0.08 & 0.00 & 0.14 & 0.20 & 97.99 \\ 0.26 & 0.15 & 0.05 & 0.13 & 0.20 & 97.88 \\ 0.25 & 0.11 & 0.06 & 0.12 & 0.21 & 96.86 \\ 0.27 & 0.11 & 0.00 & 0.12 & 0.19 & 97.93 \\ 0.28 & 0.13 & 0.00 & 0.12 & 0.20 & 97.20 \\ 0.25 & 0.10 & 0.10 & 0.10 & 0.22 & 98.34 \\ 0.26 & 0.14 & 0.06 & 0.12 & 0.18 & 97.76 \\ 0.24 & 0.11 & 0.00 & 0.13 & 0.19 & 97.71 \\ 0.27 & 0.12 & 0.04 & 0.12 & 0.23 & 98.01 \\ 0.27 & 0.15 & 0.00 & 0.14 & 0.18 & 97.58 \\ 0.27 & 0.14 & 0.08 & 0.13 & 0.19 & 97.86 \\ 0.26 & 0.09 & 0.00 & 0.13 & 0.19 & 98.02 \\ 0.25 & 0.09 & 0.03 & 0.11 & 0.17 & 98.00 \\ 0.26 & 0.07 & 0.00 & 0.12 & 0.20 & 98.20 \\ 0.26 & 0.13 & 0.02 & 0.15 & 0.21 & 97.90 \\ 0.26 & 0.13 & 0.05 & 0.13 & 0.21 & 98.10 \\ 0.26 & 0.10 & 0.16 & 0.13 & 0.19 & 97.93 \\ 0.25 & 0.13 & 0.00 & 0.11 & 0.20 & 97.79 \\ 0.26 & 0.14 & 0.00 & 0.12 & 0.20 & 97.83 \\ 0.28 & 0.08 & 0.02 & 0.13 & 0.17 & 97.86 \\ 0.28 & 0.16 & 0.12 & 0.14 & 0.21 & 98.16 \\ 0.28 & 0.13 & 0.00 & 0.13 & 0.20 & 97.19 \\ 0.27 & 0.15 & 0.04 & 0.11 & 0.18 & 97.71 \\ 0.24 & 0.14 & 0.00 & 0.13 & 0.24 & 97.80 \\ 0.26 & 0.06 & 0.00 & 0.13 & 0.18 & 98.30 \\ 0.27 & 0.13 & 0.00 & 0.13 & 0.16 & 97.16 \\ 0.24 & 0.09 & 0.00 & 0.10 & 0.20 & 98.15 \\ 0.25 & 0.11 & 0.00 & 0.13 & 0.21 & 97.70 \\ 0.25 & & 0.00 & 0.13 & 0.19 & 97.89 \\ 0.27 & & 0.00 & 0.13 & 0.24 & 97.66 \\ 0.25 & & 0.00 & 0.13 & 0.20 & 97.55 \\ 0.21 & & 0.00 & 0.13 & 0.19 & 97.71 \\ 0.00 & 0.10 & 0.19 & 98.57 \\ 0.19 & 0.00 & 0.14 & 0.19 & 97.92 \\ 0.13 & 0.00 & 0.13 & 0.18 & 97.94\end{array}$




$\begin{array}{lllll}0.28 & 0.00 & 0.13 & 0.17 & 97.90 \\ 0.24 & 0.15 & 0.13 & 0.23 & 98.39 \\ 0.26 & 0.08 & 0.13 & 0.24 & 98.42 \\ 0.24 & 0.00 & 0.15 & 0.20 & 98.27 \\ 0.24 & 0.02 & 0.12 & 0.19 & 98.61 \\ 0.26 & 0.00 & 0.15 & 0.21 & 97.30 \\ 0.26 & 0.07 & 0.15 & 0.20 & 97.06 \\ 0.25 & 0.03 & 0.15 & 0.21 & 97.84 \\ 0.27 & 0.03 & 0.13 & 0.20 & 97.17 \\ 0.28 & 0.01 & 0.15 & 0.22 & 97.73 \\ 0.26 & 0.00 & 0.13 & 0.23 & 97.64 \\ 0.27 & 0.00 & 0.13 & 0.21 & 96.00 \\ 0.26 & 0.03 & 0.14 & 0.20 & 97.73 \\ 0.24 & 0.00 & 0.14 & 0.23 & 98.13 \\ 0.26 & 0.00 & 0.15 & 0.19 & 97.82 \\ 0.25 & 0.00 & 0.12 & 0.18 & 98.00 \\ 0.26 & 0.04 & 0.14 & 0.21 & 97.51 \\ 0.25 & 0.00 & 0.13 & 0.21 & 97.10 \\ 0.27 & 0.09 & 0.13 & 0.20 & 98.03 \\ 0.27 & 0.00 & 0.14 & 0.20 & 97.37 \\ 0.26 & 0.00 & 0.13 & 0.18 & 97.78 \\ 0.25 & 0.17 & 0.13 & 0.17 & 98.61 \\ 0.26 & 0.08 & 0.14 & 0.20 & 98.02 \\ 0.28 & 0.11 & 0.17 & 0.16 & 97.74 \\ 0.25 & 0.00 & 0.16 & 0.16 & 97.45 \\ 0.26 & 0.00 & 0.16 & 0.18 & 97.68 \\ 0.24 & 0.00 & 0.15 & 0.15 & 97.40 \\ 0.25 & 0.00 & 0.15 & 0.17 & 97.68 \\ 0.24 & 0.02 & 0.15 & 0.17 & 98.23 \\ 0.25 & 0.00 & 0.15 & 0.19 & 97.90 \\ 0.26 & 0.02 & 0.16 & 0.18 & 97.54\end{array}$

